\title{
Stem cells in plastic and regenerative surgery
}

Citation for published version (APA):

Beugels, J. (2020). Stem cells in plastic and regenerative surgery: immunomodulation and angiogenesis. [Doctoral Thesis, Maastricht University]. ProefschriftMaken. https://doi.org/10.26481/dis.20200916jb

Document status and date:

Published: 01/01/2020

DOI:

10.26481/dis.20200916jb

Document Version:

Publisher's PDF, also known as Version of record

\section{Please check the document version of this publication:}

- A submitted manuscript is the version of the article upon submission and before peer-review. There can be important differences between the submitted version and the official published version of record.

People interested in the research are advised to contact the author for the final version of the publication, or visit the DOI to the publisher's website.

- The final author version and the galley proof are versions of the publication after peer review.

- The final published version features the final layout of the paper including the volume, issue and page numbers.

Link to publication

\footnotetext{
General rights rights.

- You may freely distribute the URL identifying the publication in the public portal. please follow below link for the End User Agreement:

www.umlib.nl/taverne-license

Take down policy

If you believe that this document breaches copyright please contact us at:

repository@maastrichtuniversity.nl

providing details and we will investigate your claim.
}

Copyright and moral rights for the publications made accessible in the public portal are retained by the authors and/or other copyright owners and it is a condition of accessing publications that users recognise and abide by the legal requirements associated with these

- Users may download and print one copy of any publication from the public portal for the purpose of private study or research.

- You may not further distribute the material or use it for any profit-making activity or commercial gain

If the publication is distributed under the terms of Article $25 \mathrm{fa}$ of the Dutch Copyright Act, indicated by the "Taverne" license above, 
Stem cells in plastic and regenerative surgery: Immunomodulation and angiogenesis

Jip Beugels

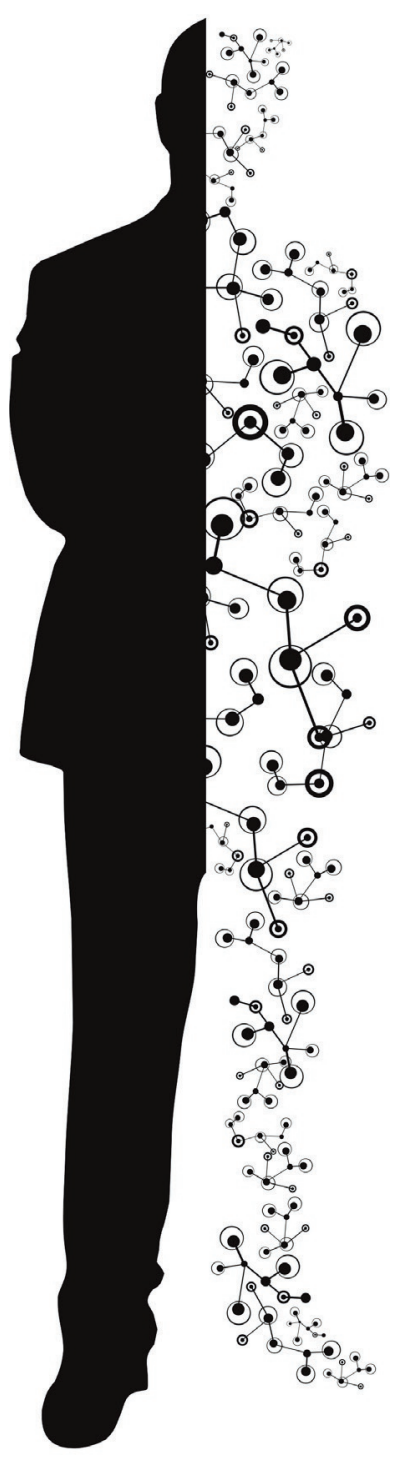



Stem cells in plastic and regenerative surgery:

Immunomodulation and angiogenesis

Jip Beugels 


\section{Colofon}

(C) Copyright Jip Beugels, Maastricht, 2020

All rights reserved. No part of this book may be reproduced or transmitted in any form or by any means, without prior permission in writing by the author, or when appropriate, by the publishers of the publications.

ISBN: 978-94-6380-941-2

Cover and chapter design, lay-out and printing by:

ProefschriftMaken | www.proefschriftmaken.nl

This thesis was financially supported by Maastricht University through the School of Nutrition and Translational Research in Metabolism (NUTRIM), Maastricht university Medical Center (MUMC+), Neuroplast Stem Cell Technology and Junior Vereniging Plastische Chirurgie (JVPC).

\section{Maastricht UMC+}

Maastricht University
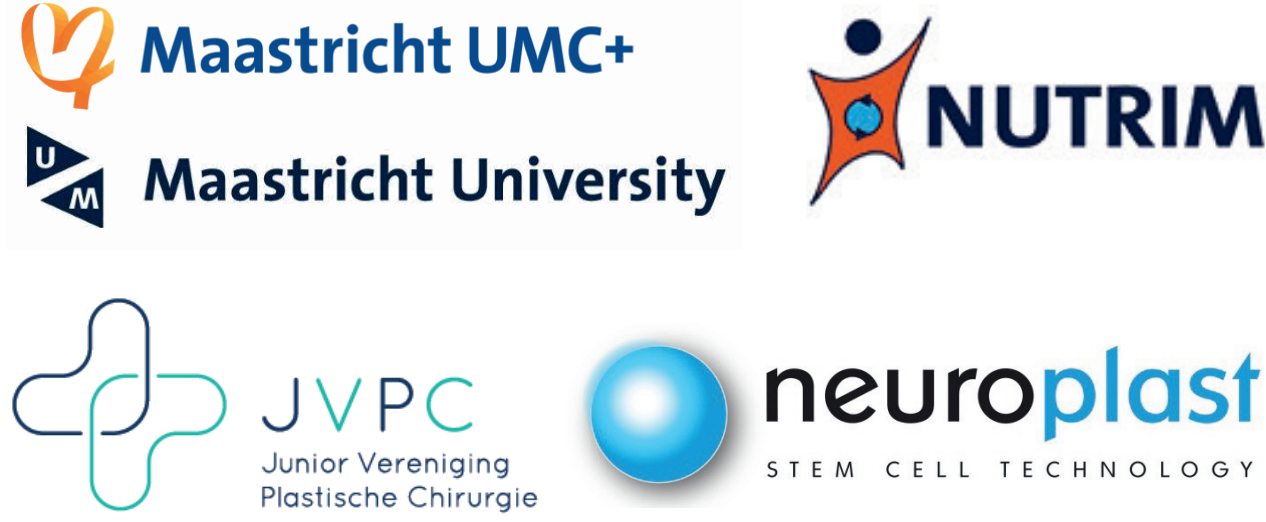

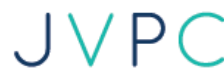

Junior Vereniging Plastische Chirurgie

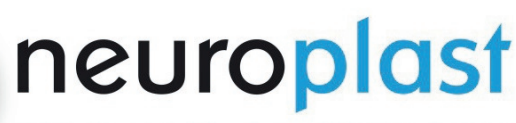

STEM CELL TECHNOLOGY 


\title{
Stem cells in plastic and regenerative surgery:
}

\section{Immunomodulation and angiogenesis}

\author{
PROEFSCHRIFT \\ ter verkrijging van de graad van doctor aan de Universiteit Maastricht, \\ op gezag van de Rector Magnificus,
}

Prof. dr. Rianne M. Letschert

volgens het besluit van het College van Decanen,

in het openbaar te verdedigen

op woensdag 16 september 2020 om 12.00 uur

door

Jip Beugels 


\section{PROMOTORES}

Prof. dr. R.R.W.J. van der Hulst

Prof. dr. B.W. Kramer

\section{COPROMOTOR}

Dr. T.G.A.M. Wolfs

\section{MEMBERS OF THE DOCTORAL COMMITTEE}

Prof. dr. F.C.S. Ramaekers (Chair)

Prof. dr. med. J.P. Beijer (Uniklinik RWTH Aachen)

Prof dr. M. van Griensven

Dr. D.I. Booi (Zuyderland Medical Center) 


\section{Contents}

Chapter 1 General introduction, aims and outline of this thesis

Chapter 2 Efficacy and safety of autologous fat transfer in facial reconstructive surgery: a systematic review and meta-analysis.

Chapter 3 Autologous fat transfer as a treatment for peripheral neuropathic pain without apparent cause

Chapter 4 Standardized human bone marrow-derived stem cells infusion improves survival and recovery in a rat model of spinal cord injury

Chapter 5 Efficacy of different doses of human adult bone marrow stem cell transplantation on angiogenesis in a rat model with hind limb ischemia

Chapter 6 Electrical stimulation promotes the angiogenic potential of adipose-derived stem cells

Chapter 7 General discussion

Chapter 8 Summary

Chapter 9 Summary in Dutch - Nederlandse samenvatting

Chapter 10 Valorization

Appendix Acknowledgements - Dankwoord

List of publications \& Curriculum Vitae 


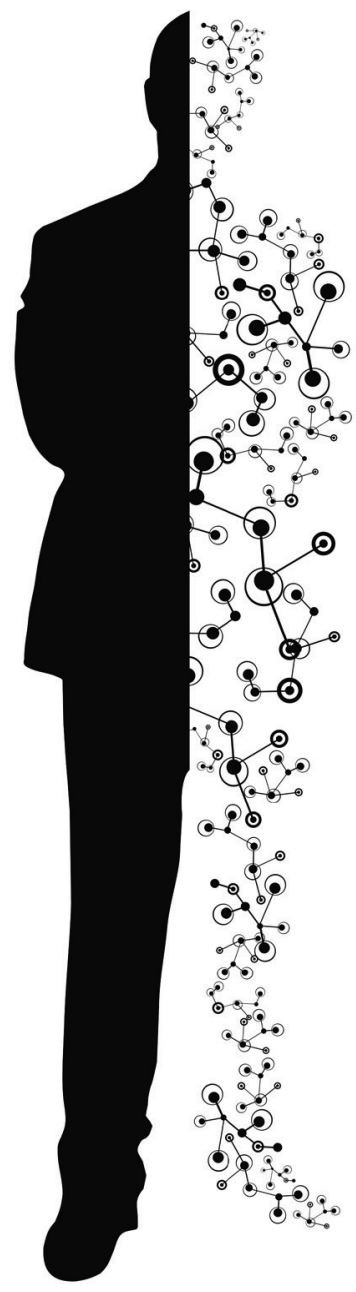




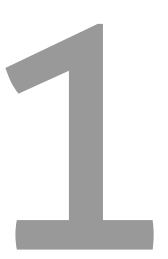

General introduction 


\section{General introduction}

"From a historical perspective, the plastic surgeon has been characterized as an innovator who is willing and ready to both introduce and to accept new ideas." Stephen J. Mathes

\section{Plastic surgery restores form and function}

Plastic surgery is a unique problem-solving specialty, which, not limited by anatomical boundaries, performs surgery from head to toe. ${ }^{1}$ Historically, plastic surgery has mostly focused on form, as implied by the term "plastic". Since the early writings of Sushruta, dating back to $600 \mathrm{BC}$ and considered by many to be the father of plastic surgery for his description of nose reconstruction using a cheek flap, surgeons have come a long way to restore or improve form in congenital and acquired defects. ${ }^{2}$ Reconstructive options include the use of autologous tissue or synthetic materials. While having the advantage of endless of-the-shelf supply and being relatively easy to place, synthetic implants also have some major drawbacks, such as suboptimal biocompatibility, poor integration with native tissue, rupture, leakage, capsular contraction, lack of natural feel and an unnatural appearance. ${ }^{3}$ Due to these shortcomings, there is a great demand for reconstruction of soft tissue defects using autologous tissue.

Through persistent anatomical research a deep knowledge of the vascular system has been developed, and with modern day technological advancements and the advent of (super) microsurgery, the array of surgical options has never been greater. While numerous different approaches have been tested and introduced, the basic principle remains the same. A flap consisting of skin, fat and sometimes muscle is carefully dissected from the donor site and transferred locally, leaving the blood supply intact or completely freed from the surrounding tissue, enabling the surgeon to transfer it to a distant recipient site. In the latter procedure (so-called free flaps) the native vascular supply is cut, allowing the flap to be lifted. Once relocated the flap's vascular pedicle is microsurgically attached to the artery and vein of the recipient site to restore blood flow through the tissue. If sensation or movement is required in free flaps, plastic surgeons are able to reconnect nerve stomps directly or through the use of an interposition graft. Regaining these functions leads to a significant improvement of the reported quality of life. ${ }^{4}$ An example of this is a recently 
described method of DIEP flap neurotization that showed promising results. ${ }^{5,6}$ In other forms of breast reconstruction, particularly in autologous fat transfer, surgical neurotization is not possible. However, reinnervation might be obtained in a different way.

\section{Autologous fat transfer}

In spite of the benefits, free flaps have certain disadvantages. Relative to implant-based reconstruction, the procedure is long and difficult, there is an increased risk of donor site morbidities and tissue necrosis, with a longer recovery period (up to six weeks to be able to perform normal physical activities) and more elaborate scars. In cases where there is merely a volume deficit, and not skin, autologous fat transfer (AFT) or autologous fat grafting aims to overcome the drawbacks of free flaps. ${ }^{7}$ It entails harvesting adipose tissue through liposuction, processing of the lipoaspirate and subsequently injecting it back into the recipient site. The whole process is less time consuming and carries relatively low risks. Instead of introducing a foreign body such as an implant to create volume, a patient's own fat is used as a filler material. Compared to other materials autologous adipose tissue is easy to access, harvest and work with, shows excellent biocompatibility and is increasingly abundant in most individuals. ${ }^{8}$ This way AFT provides form, but it has also proven to provide a functional effect in indications such as neuropathic pain caused by neuromas ${ }^{9}$, burns ${ }^{10}$, scars ${ }^{11,12}$ and post mastectomy pain syndrome. ${ }^{13,14}$ However, a major issue in AFT is that once grafted a substantial portion of the graft is resorbed. This causes the volume of the grafted fat to decrease over time, most prominently during the first 3-6 months after the procedure. Retained volumes range between 40 and $80 \% .{ }^{15-17}$ As a consequence final volume and shape are difficult to predict. Often multiple procedures are needed in order to achieve and maintain the desired end result. A generally accepted cause for the volume decrease is the lack of vascularization in the graft during the first days after fat transfer.

\section{Advanced solutions in regenerative medicine}

In order to overcome major issues such as a lack of vascularization and innervation, advanced solutions are investigated. Approaches include increasing the vascularization of the recipient site pre- and post-operatively ${ }^{18,19}$ or modifying the pro-angiogenic potential of a given therapy by adding single or multiple growth factors ${ }^{20-22}$, platelet-rich plasma ${ }^{23,24}$, regenerative cells from stromal vascular fractions ${ }^{16,17}$ or by adding isolated stem cells. ${ }^{25,26}$ 
Others have combined cell-based therapy and biomaterials through tissue engineering leading to interesting concepts so far. Yet, despite the initial development of scaffolds for reconstructing soft tissue defects ${ }^{27}$, nobody has been able to create adequately vascularized (and innervated) constructs of relevant size. ${ }^{28}$ While major advances have been made, and the idea is enticing that one day we might have off-the-shelf customizable tissue constructs, there is still much to be learned from harnessing the body's own regenerative capabilities and technology that is able to modulate these processes. Stem cells hold the promise to help in extreme cases where angiogenesis may be severely impaired after prolonged ischemia or where central/peripheral nervous injury or neurodegeneration occurred. All biological examples correspond to clinical diseases like hind limb ischemia or spinal cord injury, where pharmacological and surgical help do not address the cause of the problem nor adequately modulate the microenvironment. Since the basic problem of inflammation, angiogenesis and regeneration is the same in plastic surgery or in any other healing process, stem cells offer the opportunity to explore a possible role in the restoration of form and function in plastic surgery.

\section{Stem cells}

In regeneration, stem cells, which are undifferentiated cells present during all stages of life, play a crucial role. Not only are they the predecessors of all cells in the body, they also have an intricate role in all major processes during the healing phase after a surgical procedure, such as inflammation and angiogenesis. The ability to differentiate varies between stem cells depending on their origin and their derivation. ${ }^{29}$ The differentiation potential can vary from totipotent to unipotent cells, and the origin varies from embryonic stem cells to adult stem cells, including tissue-resident stem cells (see figure 1). The next section will focus on multipotent adult (tissue-resident) stem cells: mesenchymal and hematopoietic stem cells. 


\section{Stem Cell Hierarchy}

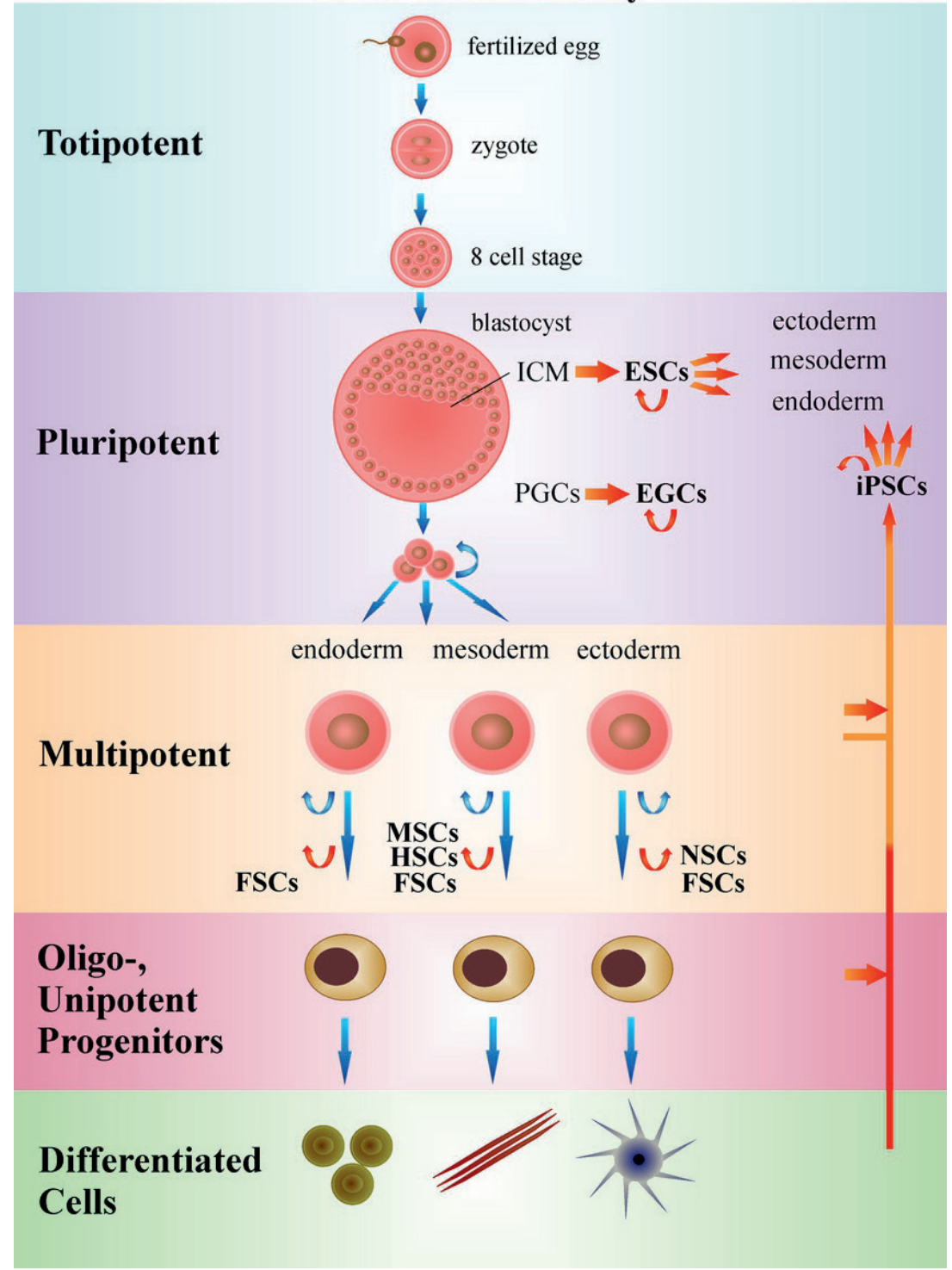

Figure 1. Stem cell hierarchy. ${ }^{30} \mathrm{ICM}=$ Inner Cell Mass. ESC $=$ Embryonic Stem Cell. $P G C=$ Primordial Germ Cell. EGC $=$ Embryonic Germ Cell. FSC $=$ Follicle Stem Cell. $M S C=$ Mesenchynal stem cell. $H S C=$ Hematopoietic Stem cell. NSC = Nerve Stem Cell. iPSC = induced Pluripotent Stem Cell. 


\section{Bone marrow-derived mesenchymal stem cells}

Bone marrow is located within the spongy or cancellous portions of bone and is composed of hematopoietic cells (myeloid and lymphoid), marrow adipose tissue and supportive stromal cells. The stroma is heterogenic in nature and contains fibroblasts, macrophages, osteoblasts, osteoclasts and endothelial (stem) cells as well as mesenchymal stem cells. Friedenstein et al. were the first the point out a subset of precursor cells for osteoblast and fibrous tissue in the late 60 's. ${ }^{31}$ These cells were adherent to plastic in culture and could form colonies of fibroblastoid cells and were initially dubbed colony-forming-unit-fibroblasts (CFU-f). Later studies confirmed and extended these findings by delineating the multipotency and ascertain the distinction from endothelial and hematopoietic cells. ${ }^{32-34}$ Bone marrow-derived mesenchymal stem cells (BM-MSCs) are fibroblast-like cells which comprise less than $0.1 \%$ of the total population of adult bone marrow cells. ${ }^{35}$ Caplan coined the term mesenchymal stem cells (MSCs) to indicate the ability to differentiate into cells within the mesenchymal lineage ${ }^{36}$, although later studies showed they are capable of forming cell types outside the mesenchymal lineage such as vascular smooth muscle cells. ${ }^{37}$ Next to their differentiation capacity, noted attributes of BM-MSCs are migration to sites of injury or inflammation after intravenous infusion, their ability to influence other resident progenitor cells, and promote recovery of damaged tissues through secretion of a variety of cytokines and chemokines. $^{38,39}$ It is not surprising that BM-MSC have already been used clinically to treat a wide range of conditions and diseases, like myocardial infarction ${ }^{40}$, stroke $^{41}$, amyotrophic lateral sclerosis ${ }^{42}$, Graft-Versus-Host-Disease after bone marrow transplantation ${ }^{43}$, chronic wounds ${ }^{44}$ and several others.

\section{Hematopoietic stem cells}

Hematopoietic stem cells (HSCs) give rise to myeloid and lymphoid lineages leading to the production of red blood cells, white blood cells and platelets in a process called hematopoiesis which forms approximately $10^{10}$ cells daily. ${ }^{45}$ They were first described by Till and McCulloch in 1961, yet their exact definition has changed over time. ${ }^{46}$ An extraordinary feature of these cells is their ability to self-renew, even at extremely high turnover rates. Two types of HSC have been described: long-term CD34 ${ }^{+}$repopulating cells (1/100.000 cells in marrow) which remain active and functional throughout life, and short-term repopulating 
cells that remain for about 6 weeks. ${ }^{47}$ Another feature of HSC is that they too are able to across lineages when exposed to specific stimuli. ${ }^{48}$ HSCs reside in two distinct immuneprivileged niches or locations in the bone marrow and are heavily influenced by the microenvironment surrounding them. ${ }^{49}$ Both the sympathetic nervous system and neighboring cells such as MSC play a role in regulation. ${ }^{50}$ Beside their clinical use in hematopoietic cell transplantation, HSC are widely investigated for their regenerative characteristics. $^{45}$

\section{Adipose derived (mesenchymal) stem cells}

Adipose tissue contains a heterogeneous collection of cells organized in a structured mesh of connective tissue that is highly vascularized (see figure 2). It has the highest tissue partial oxygen tension of any organ. ${ }^{51}$ Adipocytes make up more than 90 percent of the volume due to their enormous size of 50 to $130 \mu \mathrm{m}$. However, they only represent 20 percent or less in total number of cells. ${ }^{52}$ The other cells present in fat, called stromal vascular fraction (SVF), comprise primarily of pre-adipocytes/adipose-derived stromal/stem cells, immune cells (macrophages, granulocytes and lymphocytes), endothelial (progenitor) cells, pericytes (progenitor) cells and fibroblasts. ${ }^{52,53}$

A

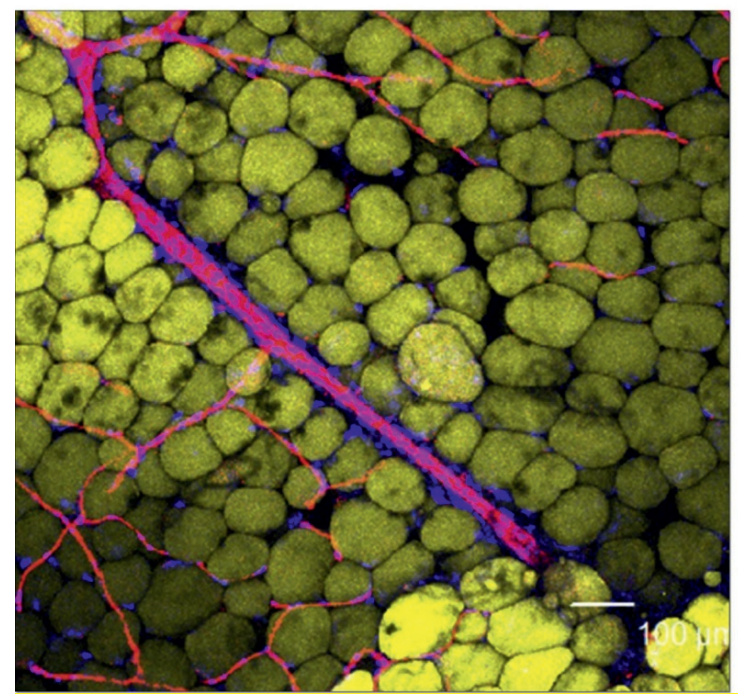

B

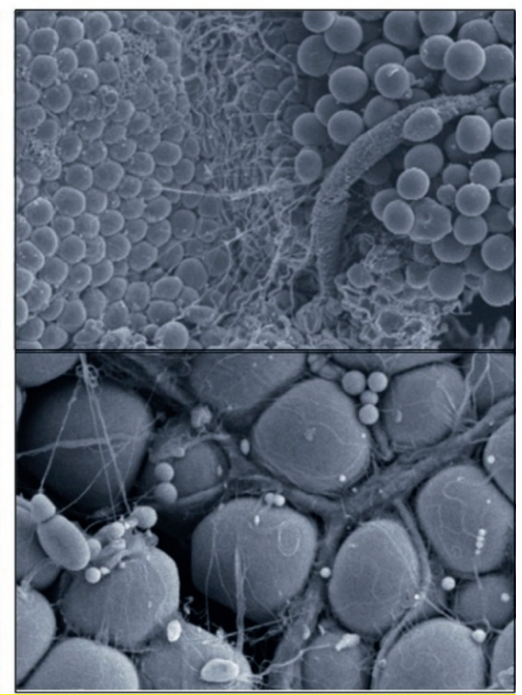

Figure 2. Structure of human adipose tissue. Immunohistologic image on the left, further detailed by the electromicroscopic images on the right. ${ }^{51}$ 
Adipose-derived stem cells are adult stem cells of mesenchymal origin native to adipose tissue. While described as pre-adipocytes in several papers 4 decades ago ${ }^{54,55}$, the term "adipose-derived stromal cells (ADSC)" was coined in several pioneering studies in the early 2000 's. ${ }^{56-58}$ Later, due to confusion over the number of different names to identify the same adipose tissue cell population, the International Fat Applied Technology Society reached a consensus to adopt the term "adipose-derived stem cell (ASC)" for further use in literature. ${ }^{59}$ ASC are classically described to be able to differentiate into cells of the adipogenic, osteogenic, and chondrogenic lineages, while for example also myogenic and neural differentiation have been described. ${ }^{60,61}$ Although different techniques are available to isolate ASC, adipose tissue is generally harvested through liposuction or excision, and enzymatically digested to isolate the SVF. The SVF is then sorted by flow cytometry or directly plated in culture flasks, making use of the characteristic of ASC to adhere to plastic, while other cells from the SVF are washed away in subsequent medium changes. Human ASC have a typical fibroblast-like appearance in culture and can be phenotypically characterized by the following markers: $\mathrm{CD} 45^{-} \mathrm{CD} 235 \mathrm{a}^{-} \mathrm{CD} 31^{-} \mathrm{CD} 34^{+}$with added markers in vitro of $\mathrm{CD} 13$, CD73, CD90 and CD105. ${ }^{62}$ They can be distinctly separated from endothelial $\left(\mathrm{CD} 31^{+}\right)$and hematopoietic $\left(\mathrm{CD} 45^{+}\right)$cells in the SVF. Furthermore, ASC lack expression of HLA-DR and costimulatory molecules, leading them to be immune privileged. Benefits of ASC over their mesenchymal counterpart the "bone marrow-derived MSC (BM-MSC)" include ease of isolation, higher abundance of tissue itself and higher cell yields as 1 gram of adipose tissue yields approximately 5000 stem cells, which is a factor of 100-500 higher than MSCs from bone marrow. ${ }^{63,64}$ The exact location of the ASC within non-disturbed fat remains highly debated, but multiple studies have shown that ASC may reside within the perivascular tissue. ${ }^{65,66}$ ASC have been shown to function in a dual way: by transdifferentiation into mature cells and via paracrine mechanisms involving neighboring cells. While the first hypotheses on the mechanism of action of ASC focused towards differentiation, secretion of a myriad of cytokines and growth factors may be more important than previously thought. ${ }^{67}$ Numerous studies have investigated the secretome of ASC, and found amongst others that they possess robust pro-angiogenic characteristics and immunomodulatory properties. ${ }^{57,60,62}$ 


\section{What happens in the graft after fat transfer?}

The moment the adipose tissue is removed from the donor site it becomes ischemic. The liposuction process itself causes injury to the donor tissue as well as the recipient site. A cocktail of injury related cytokines, such as basic fibroblast growth factor and vascular endothelial growth factor, are released triggering a cascade of healing processes. ${ }^{51}$ Meanwhile, inflammatory cells such as M1 macrophages and lymphocytes home in on the grafted area and release pro-inflammatory cytokines. M1 (or "classically activated) macrophages are formed in response to several inflammatory mediators such as IFN- $\gamma$ and LPS and in turn produce a high level of pro-inflammatory cytokines such as TNF- $\alpha$ and IL$6 .^{68,69}$ In this time the adipocytes struggle to survive, and the way they do this is described by two general theories. The cell survival theory was the first one published by Peer in the 1950 's. ${ }^{70}$ This theory states that adipocytes in grafts of certain size survive directly by plasmatic diffusion and early revascularization. In physiological conditions each individual fat cell is no more than 200-300 $\mu \mathrm{m}$ away from the nearest capillary, enabling oxygen and nutrients to reach the cell through diffusion. If the limit is exceeded, for example when grafting larger volumes of fat, the core of the graft is deprived of oxygen and nutrients leading to cell death. Later studies by the Yoshimura group with the use of more sophisticated technology, showed that actually most adipocytes in the fat graft die within 24 hours. ${ }^{71,72}$ The authors describe three distinguishable zones in fat grafts: the outer surviving zone in which all of the cells survive through diffusion, the middle "regenerating" zone in which only the subset of cells that are most resistant to hypoxia survive (i.e. ASC) and provide new adipocytes to replace the dead ones, and the central or "necrotic" zone in which all cells perish (see figure 3 ). After the initial inflammatory response, both resident ASC and infiltrated stem cells from bone marrow spin the micro environment more towards pro regeneration. M2 macrophages appear and surround the oil droplets that remain after adipocyte death. M2 (or "alternatively activated") macrophages show antiinflammatory/regenerative properties, are induced through $\mathrm{IL}-4$ and IL-13 and are characterized by for example increased production of IL-10. ${ }^{69}$ In the following time period new adipocytes mature and vascular networks are reestablished. If the oil droplets are not successfully phagocytized by the macrophages, M2 macrophages will either form fibrotic tissue or encapsulate the oil, forming a cyst. ${ }^{73}$ 

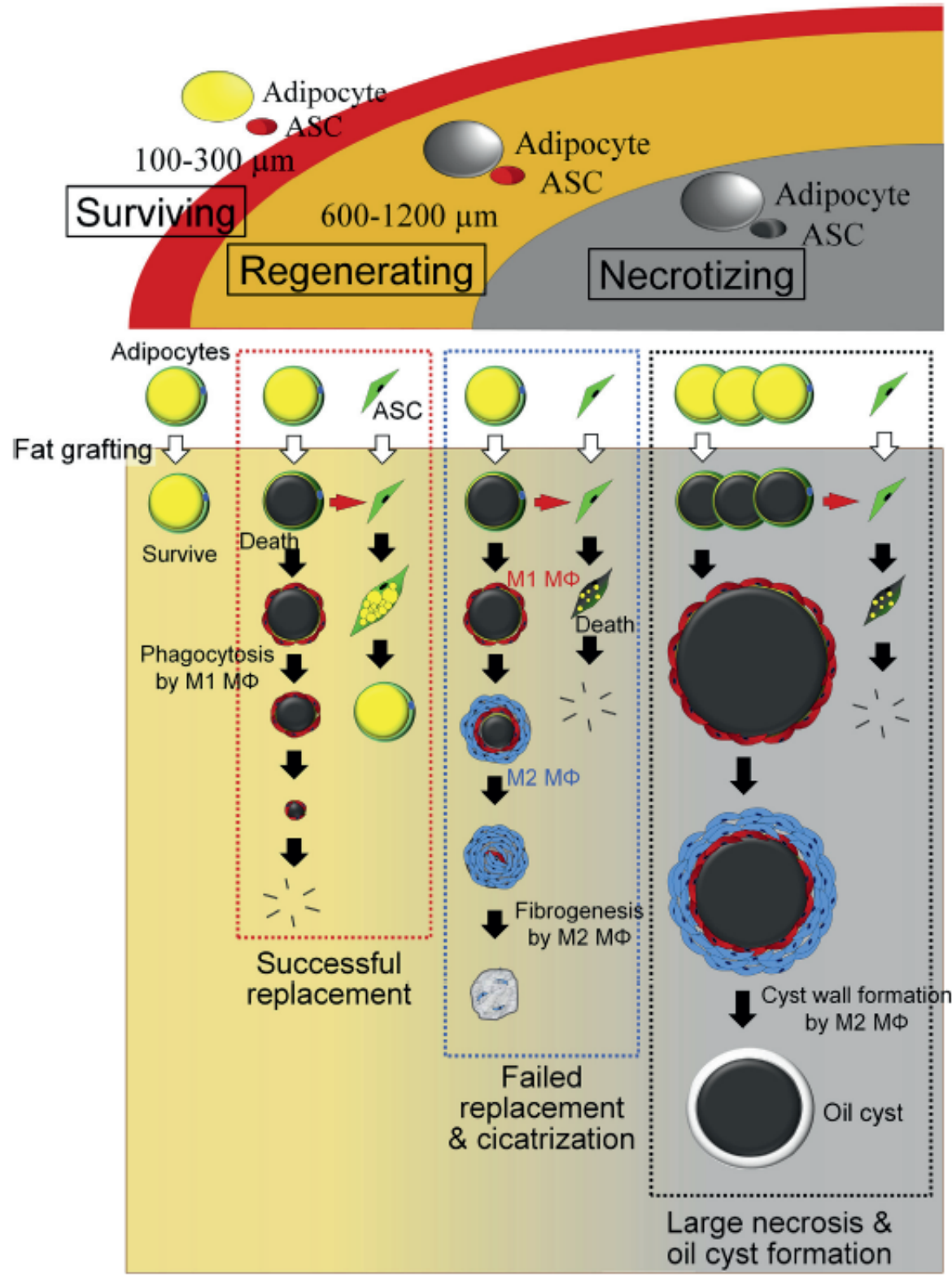

Figure 3. The three zones in fat grafts and an overview of the events in the graft following fat transfer. ${ }^{51}$ 


\section{Angiogenesis}

Angiogenesis describes the chain of events leading to the formation of new capillary networks and is key in development and regeneration after injury. It is the focus of treatments of ischemia-related illnesses and (chronic) wounds. Therapies aim to revascularize a certain area of tissue by sprouting capillaries of existing collateral bloods vessels. This applies certainly to fat grafts, in which an avascular graft has to be revascularized to maintain the tissue long term. Three pioneering studies from separate groups in 2004 sparked interest in the pro-angiogenic effect of ASC. Rehman et al showed that ASC reacted to hypoxia in cell culture with increased levels of secreted VEGF by 5 fold, and that conditioned medium (CM) of hypoxic ASC had a beneficial effect on the growth and apoptosis rate of endothelial cells (EC). ${ }^{74}$ In the same year it was shown that human ASC assumed endothelial-like functionality in vitro and in vivo by using hind limb ischemia models. ${ }^{75,76}$ The hind limb ischemia model was later also used in a comparative study of murine ASC vs BM-MSC, adipocytes and PBS. ${ }^{77}$ Herein, injection of ASC after vessel ligation proved to promote angiogenesis from collateral vessels, enabling better tissue perfusion and increasing capillary density. Although some level of transdifferentiation towards adipocytes was observed more emphasis was placed on the increased expression of VEGF. While VEGF is a key pro-angiogenic factor, it is unlikely to be the only factor of importance. Exploratory studies into the secretome of ASC revealed a plethora of other angiogenesis related factors such as hepatocyte growth factor (HGF), basic fibroblast growth factor (bFGF/FGF-2), transforming growth factor $\beta 2$ (TGF- $\beta 2$ ), placental growth factor (PGF) and angiopoietins 1 and 2 (Ang-1 and Ang-2). ${ }^{78}$ Interestingly the secretion of these factors varied after supplementation of different additives to the culture medium, hinting at an adaptive secretory profile based on the current state of the local tissue environment at any given time. Equally interesting was the fact that neutralizing VEGF and HGF in the CM led to decreased viability and migration of endothelial cells (EC). Similar effects have been seen after silencing expression of for example HGF. Cai et al found that this lead to a decreased ability to promote survival, proliferation and migration of both mature and progenitor EC in vitro and decreased reperfusion in the ischemic hind limb model in vivo. ${ }^{79}$

More specifically in fat grafting, ASC have been shown to promote angiogenesis during the acute phase after surgery and form microtubules within $24 \mathrm{hr}$ of ischemia and capillary 
networks within 3 days. ${ }^{80,81}$ Suga and colleagues performed an insightful study in which ASC were injected into inguinal fat pads of mice. ${ }^{82}$ The fluorescently labeled ASC were tracked through bioluminescence in the first 28 days after surgery, and although more ASC were present in ischemic fat pads at day 7 versus normal fat pads, very little luminescent cells were traced after 28 days indicating cell death. However, proliferating EC were seen adjacent to the graft, which lead to the hypothesis that paracrine mechanisms might play a role. This hypothesis was confirmed by the finding of elevated levels of VEGF and HGF in the tissue lysates after 7 days, and in vitro data that revealed a stimulatory effect of ASC in EC proliferation and network assays, which was inhibited when neutralizing antibodies against VEGF or HGF were added.

ASC have also been incorporated into tissue engineering, a relatively new field in which cell therapy is often combined with biomaterials. Yet, despite the initial development of scaffolds for reconstructing soft tissue defects ${ }^{27}$, so far it is impossible to create adequately vascularized constructs of relevant size ${ }^{28}$. The resulting lack of oxygen and nutrients prevents formation of a habitable environment for cells to reside in. While a multitude of techniques have been employed in the past to vascularize scaffolds ${ }^{83-85}$, a truly successful method for large volume constructs has yet to be developed.

\section{Immunomodulation by MSCs}

MSCs have been praised for their ability to influence the immune system and have therefore been widely used as therapeutic agents for the treatment of autoimmune diseases. However, MSCs are not constitutively immunosuppressive. The immunomodulatory characteristics are strongly influenced by signals from the surrounding microenvironment. MSCs need 'priming' by specific cytokines such as TNF- $\alpha$ to become immunosuppressive and the level of inflammation and concentration of these cytokines in the local microenvironment at the time of administration plays a critical role in determining the final outcome. $^{86,87}$

There are several ways for MSCs to mediate their effect on the immune system, ranging from cell to cell contact to the production of paracrine factors such as cytokines and growth factors $^{88}$. Moreover, MSCs regulate proliferation, activation and effector functions of 
immune cells (macrophages, dendritic cells (DCs), natural killer (NK) and natural killer T (NKT) cells, neutrophils, basophils, eosinophils, mast cells, T and B lymphocytes), indicating their therapeutic potential in the treatment of autoimmune and inflammatory diseases. ${ }^{88}$

During the early phase of inflammation MSCs promote recruitment of neutrophils to the site of inflammation, protect them from apoptosis and may supercharge their ability to phagocytize bacteria. ${ }^{89,90}$ This process is sustained by several factors that are release upon recognition of microbial antibodies by (tissue-resident) MSCs, such as IL-8 and macrophage migration inhibitory factor (MIF). ${ }^{91}$ However, during the late stage of the immune response even in the presence of high levels of TNF- $\alpha$ and IFN- $\gamma$, MSCs may induce the production of anti-inflammatory cytokines in monocytes/macrophages such as IL-10, inhibiting neutrophil migration and consequent oxidative damage. ${ }^{92}$ These findings suggest a direct regulatory role, in which MSCs serve to promote host defense on one hand, and at the same time prevent tissue damage by an excessive immune response, and focus on tissue regeneration..$^{93}$

\section{Electrical stimulation}

In the human body various biochemical and biophysical cues are responsible for the creation and maintenance of tissues and organs. ${ }^{94}$ Among these are endogenous electric fields (EFs), which are naturally present in living organisms. Besides the well-known role of electricity in generating action potentials in nerves, EFs also play an important role in physiological processes such as embryonic development, regeneration and wound healing. ${ }^{95,96}$ EFs are generated through constant movement of negatively and positively charged ions across channels in the membrane of individual cells leading to a membrane potential. Analogous to the membrane potential on cell level is the macro-equivalent that occurs when cells are together in a continuous layer, establishing a tissue-level electric gradient across the cellular interface. ${ }^{97}$ An example of this is the trans-epithelial potential found in skin, lens and cornea. ${ }^{98}$ In case of a breach of the epithelium, such as in wounds, the potential difference between the two sides of the epithelium short circuits, generating an EF that is responsible for cell migration towards the injury site and controls cellular actions like spatial orientation and cell division rate. ${ }^{99-102}$ 
Building upon this knowledge, previous in vitro and in vivo studies have applied some form of electrical stimulation (ES), not only to accelerate wound healing but also to influence a variety of cells and signaling cascades. There are many types of electrical stimulation, varying from applied direct current (DC) to more complex stimuli (either using direct or alternating current) in various wave forms and stimuli patterns, generated by a multitude of chips, generators or therapeutic systems. ${ }^{94}$ There are also several ways to deliver the electrical stimulus, ranging from direct stimulation/coupling to indirect stimulation/coupling (capacitive coupling, inductive coupling such as pulsed electromagnetic fields (PEMF), or a combination of both).

Studies so far have shown impressive results in topics such as wound healing ${ }^{103}$, bone healing ${ }^{104}$ and angiogenesis ${ }^{105}$. Sheikh et al demonstrated for example that ES of ischemic rabbit hind limbs significantly induced both arteriogenesis and angiogenesis ${ }^{106}$. The exact mechanisms remain to be elucidated, although in endothelial cells the enhanced release of VEGF and activation of VEGF receptors (VEGFRs), phosphatidylinositol-3-kinase (PI3K)-Akt and Rho-ROCK elements of the VEGFR signaling pathway play a significant role. ${ }^{107}$ That same VEGF upregulation after ES was found in BM-MSCs ${ }^{108}$ and ASCs. ${ }^{109}$ Clinical applications of ES, focusing specifically on modifying or amplifying the regenerative effects of stem cells could be a useful add-on therapy to existing treatment regimens. 


\section{Aim of this thesis}

The aim of this thesis was to investigate the effect of stem cells on the two most important processes that play a role in regeneration after (surgical) tissue trauma: inflammation and angiogenesis. A deeper understanding of the intricate sequence of events and the role of stem cells herein can lead to new surgical approaches and/or post-operation management. Grafting of autologous fat is an important tool in the plastic surgeon's armamentarium, however a partial resorption of the graft remains a problem. The safety and effectiveness of stem cells as well as a clinical example of the regenerative effects of adipose tissue in neuropathic pain were studied. In the end of this thesis we performed a proof of principle study with the aim to develop a non-invasive, safe and low-cost technique to modify the behavior of adipose-derived stem cells with electrical stimulation, specifically focused towards improving autologous fat transfer.

\section{Outline of this thesis}

Chapter 2. Efficacy and Safety of Autologous Fat Transfer in Facial Reconstructive Surgery: A Systematic Review and Meta-analysis.

In chapter 2, we focused specifically on autologous fat transfer thorough analysis of the efficacy and safety of performing autologous fat transfer in plastic surgery. We compiled the available clinical data in a systematic review and meta-analysis.

\section{Chapter 3. Autologous Fat Transfer as a Treatment for Peripheral Neuropathic Pain} without Apparent Cause.

In chapter 3 , we tested in a clinical trial whether patients in a chronic neuropathic pain setting would benefit from autologous fat transfer with respect to their pain perception, possibly through the regenerative capabilities of cells within the transplant.

\section{Chapter 4. Standardized Human Bone Marrow-derived Stem Cells Infusion Improves} Survival and Recovery in a Rat Model of Spinal Cord Injury.

In chapter 4, we set out to investigate the immunomodulatory properties of a refined bonemarrow derived stem cell preparation of MSCs and HSCs in an acute model of spinal cord 
injury. Our focus lay towards researching the anti-inflammatory properties and the effect on nerve regeneration in the context of improved functional outcome.

Chapter 5. Efficacy of Different Doses of Human Autologous Adult Bone Marrow Stem Cell Transplantation on Angiogenesis in a Rat Model With Hind Limb Ischemia.

In chapter 5, we administered bone marrow-derived stem cells intramuscularly and intraarterially in a rat hind limb ischemia model in order to stimulate angiogenesis. The surgical ligation of arterial blood vessels lead to an acute-onset traumatic ischemia, which enabled us to directly visualize the angiogenic effects of the administered stem cells.

Chapter 6. Electrical Stimulation Promotes the Angiogenic Potential of Adipose-derived Stem Cells.

In chapter 6, we electrically stimulated adipose tissue-derived stem cells in vitro to determine the angiogenic effect of the conditioned medium in an in vivo animal model. This pilot study lays the foundation for further translational studies. 


\section{Rererences}

1 Mathes, S. J. \& Hentz, V. R. Plastic Surgery. (Saunders Elsevier, 2006).

2 Champaneria, M. C., Workman, A. D. \& Gupta, S. C. Sushruta: father of plastic surgery. Annals of plastic surgery 73, 2-7, doi:10.1097/SAP.0b013e31827ae9f5 (2014).

3 Miller, M. J. \& Patrick, C. W., Jr. Tissue engineering. Clinics in plastic surgery 30, 91-103, vii, doi:10.1016/s0094-1298(02)00071-8 (2003).

4 Temple, C. L. et al. Sensibility following innervated free TRAM flap for breast reconstruction: Part II. Innervation improves patient-rated quality of life. Plastic and reconstructive surgery 124, 1419-1425, doi:10.1097/PRS.0b013e3181b98963 (2009).

5 Spiegel, A. J., Menn, Z. K., Eldor, L., Kaufman, Y. \& Dellon, A. L. Breast Reinnervation: DIEP Neurotization Using the Third Anterior Intercostal Nerve. Plastic and reconstructive surgery. Global open 1, e72, doi:10.1097/gox.0000000000000008 (2013).

6 Beugels, J. et al. Sensory Recovery of the Breast following Innervated and Noninnervated DIEP Flap Breast Reconstruction. Plastic and reconstructive surgery 144, 178e-188e, doi:10.1097/prs.0000000000005802 (2019).

7 Delay, E., Garson, S., Tousson, G. \& Sinna, R. Fat injection to the breast: technique, results, and indications based on 880 procedures over 10 years. Aesthetic surgery journal 29, 360376, doi:10.1016/j.asj.2009.08.010 (2009).

8 Coleman, S. R. Structural fat grafting: more than a permanent filler. Plastic and reconstructive surgery 118, 108S-120S, doi:10.1097/01.prs.0000234610.81672.e7 (2006).

9 Vaienti, L., Merle, M., Battiston, B., Villani, F. \& Gazzola, R. Perineural fat grafting in the treatment of painful end-neuromas of the upper limb: a pilot study. The Journal of hand surgery, European volume 38, 36-42, doi:10.1177/1753193412441122 (2013).

10 Fredman, R., Edkins, R. E. \& Hultman, C. S. Fat Grafting for Neuropathic Pain After Severe Burns. Annals of plastic surgery 76 Suppl 4, S298-303, doi:10.1097/sap.0000000000000674 (2016).

11 Ulrich, D., Ulrich, F., van Doorn, L. \& Hovius, S. Lipofilling of perineal and vaginal scars: a new method for improvement of pain after episiotomy and perineal laceration. Plastic and reconstructive surgery 129, 593e-594e, doi:10.1097/PRS.0b013e3182419c2c (2012).

12 Huang, S. H. et al. Alleviation of neuropathic scar pain using autologous fat grafting. Annals of plastic surgery 74 Suppl 2, S99-104, doi:10.1097/sap.0000000000000462 (2015).

13 Caviggioli, F., Maione, L., Forcellini, D., Klinger, F. \& Klinger, M. Autologous fat graft in postmastectomy pain syndrome. Plastic and reconstructive surgery 128, 349-352, doi:10.1097/PRS.0b013e31821e70e7 (2011).

14 Maione, L. et al. Autologous fat graft in postmastectomy pain syndrome following breast conservative surgery and radiotherapy. Aesthetic plastic surgery 38, 528-532, doi:10.1007/s00266-014-0311-9 (2014).

15 Gir, P. et al. Fat grafting: evidence-based review on autologous fat harvesting, processing, reinjection, and storage. Plastic and reconstructive surgery 130, 249-258, doi:10.1097/PRS.0b013e318254b4d3 (2012).

16 Zhu, M. et al. Supplementation of fat grafts with adipose-derived regenerative cells improves long-term graft retention. Annals of plastic surgery 64, 222-228, doi:10.1097/SAP.0b013e31819ae05c (2010).

17 Liu, B. et al. The adjuvant use of stromal vascular fraction and platelet-rich fibrin for autologous adipose tissue transplantation. Tissue engineering. Part C, Methods 19, 1-14, doi:10.1089/ten.TEC.2012.0126 (2013).

18 Khouri, R. K. et al. Brava and autologous fat transfer is a safe and effective breast augmentation alternative: results of a 6-year, 81-patient, prospective multicenter study. Plastic and reconstructive surgery 129, 1173-1187, doi:10.1097/PRS.0b013e31824a2db6 (2012). 
Sezgin, B. et al. Improving fat graft survival through preconditioning of the recipient site with microneedling. Journal of plastic, reconstructive \& aesthetic surgery : JPRAS 67, 712-720, doi:10.1016/j.bjps.2014.01.019 (2014).

Nakamura, S. et al. Increased survival of free fat grafts and vascularization in rats with local delivery of fragmin/protamine microparticles containing FGF-2 (F/P MP-F). Journal of biomedical materials research. Part B, Applied biomaterials 96, 234-241, doi:10.1002/jbm.b.31757 (2011).

21 Craft, R. O. et al. Effect of local, long-term delivery of platelet-derived growth factor (PDGF) on injected fat graft survival in severe combined immunodeficient (SCID) mice. Journal of plastic, reconstructive \& aesthetic surgery : JPRAS 62, 235-243, doi:10.1016/j.bjps.2007.11.017 (2009).

22 Shoshani, O. et al. The effect of interleukin-8 on the viability of injected adipose tissue in nude mice. Plastic and reconstructive surgery 115, 853-859, doi:10.1097/01.prs.0000153036.71928.30 (2005).

23 Pires Fraga, M. F. et al. Increased survival of free fat grafts with platelet-rich plasma in rabbits. Journal of plastic, reconstructive \& aesthetic surgery : JPRAS 63, e818-822, doi:10.1016/j.bjps.2010.07.003 (2010).

24 Por, Y. C. et al. Platelet-rich plasma has no effect on increasing free fat graft survival in the nude mouse. Journal of plastic, reconstructive \& aesthetic surgery : JPRAS 62, 1030-1034, doi:10.1016/j.bjps.2008.01.013 (2009).

25 Piccinno, M. S. et al. Adipose stromal/stem cells assist fat transplantation reducing necrosis and increasing graft performance. Apoptosis : an international journal on programmed cell death 18, 1274-1289, doi:10.1007/s10495-013-0878-7 (2013).

26 Lu, F. et al. Improvement of the survival of human autologous fat transplantation by using VEGF-transfected adipose-derived stem cells. Plastic and reconstructive surgery 124, 14371446, doi:10.1097/PRS.0b013e3181babbb6 (2009).

27 Melchels, F. et al. CAD/CAM-assisted breast reconstruction. Biofabrication 3, 034114, doi:10.1088/1758-5082/3/3/034114 (2011).

28 Stosich, M. S. et al. Bioengineering strategies to generate vascularized soft tissue grafts with sustained shape. Methods (San Diego, Calif.) 47, 116-121, doi:10.1016/j.ymeth.2008.10.013 (2009).

29 Kolios, G. \& Moodley, Y. Introduction to stem cells and regenerative medicine. Respiration; international review of thoracic diseases 85, 3-10, doi:10.1159/000345615 (2013).

30 Forostyak O, D. G., Forostyak S. CNS Regenerative Medicine and Stem Cells. Opera Med Physiol Vol. 2, 55-62 (2016).

31 Friedenstein, A. J., Chailakhjan, R. K. \& Lalykina, K. S. The development of fibroblast colonies in monolayer cultures of guinea-pig bone marrow and spleen cells. Cell and tissue kinetics $\mathbf{3}$, 393-403, doi:10.1111/j.1365-2184.1970.tb00347.x (1970).

32 Prockop, D. J. Marrow stromal cells as stem cells for nonhematopoietic tissues. Science (New York, N.Y.) 276, 71-74, doi:10.1126/science.276.5309.71 (1997).

33 Pittenger, M. F. et al. Multilineage potential of adult human mesenchymal stem cells. Science (New York, N.Y.) 284, 143-147, doi:10.1126/science.284.5411.143 (1999).

34 Delorme, B. et al. Specific plasma membrane protein phenotype of culture-amplified and native human bone marrow mesenchymal stem cells. Blood 111, 2631-2635, doi:10.1182/blood-2007-07-099622 (2008).

35 Battiwalla, M. \& Hematti, P. Mesenchymal stem cells in hematopoietic stem cell transplantation. Cytotherapy 11, 503-515, doi:10.1080/14653240903193806 (2009).

36 Caplan, A. I. Mesenchymal stem cells. Journal of orthopaedic research : official publication of the Orthopaedic Research Society 9, 641-650, doi:10.1002/jor.1100090504 (1991).

37 Kurpinski, K. et al. Transforming growth factor-beta and notch signaling mediate stem cell differentiation into smooth muscle cells. Stem cells (Dayton, Ohio) 28, 734-742, doi:10.1002/stem.319 (2010). 
Mouiseddine, M. et al. Human mesenchymal stem cells home specifically to radiation-injured tissues in a non-obese diabetes/severe combined immunodeficiency mouse model. The British journal of radiology 80 Spec No 1, S49-55, doi:10.1259/bjr/25927054 (2007). Caplan, A. I. Adult mesenchymal stem cells for tissue engineering versus regenerative medicine. Journal of cellular physiology 213, 341-347, doi:10.1002/jcp.21200 (2007).

Chen, S. L. et al. Effect on left ventricular function of intracoronary transplantation of autologous bone marrow mesenchymal stem cell in patients with acute myocardial infarction. The American journal of cardiology 94, 92-95, doi:10.1016/j.amjcard.2004.03.034 (2004).

41 Bang, O. Y., Lee, J. S., Lee, P. H. \& Lee, G. Autologous mesenchymal stem cell transplantation in stroke patients. Annals of neurology 57, 874-882, doi:10.1002/ana.20501 (2005).

42 Mazzini, L. et al. Autologous mesenchymal stem cells: clinical applications in amyotrophic lateral sclerosis. Neurological research 28, 523-526, doi:10.1179/016164106x116791 (2006).

43 Sanchez-Guijo, F. et al. Sequential third-party mesenchymal stromal cell therapy for refractory acute graft-versus-host disease. Biology of blood and marrow transplantation : journal of the American Society for Blood and Marrow Transplantation 20, 1580-1585, doi:10.1016/j.bbmt.2014.06.015 (2014).

44 Yoshikawa, T. et al. Wound therapy by marrow mesenchymal cell transplantation. Plastic and reconstructive surgery 121, 860-877, doi:10.1097/01.prs.0000299922.96006.24 (2008).

45 Mosaad, Y. M. Hematopoietic stem cells: an overview. Transfusion and apheresis science : official journal of the World Apheresis Association : official journal of the European Society for Haemapheresis 51, 68-82, doi:10.1016/j.transci.2014.10.016 (2014).

46 Till, J. E. \& Mc, C. E. A direct measurement of the radiation sensitivity of normal mouse bone marrow cells. Radiation research 14, 213-222 (1961).

47 Brown, J. M. \& Weissman, I. L. Progress and prospects in hematopoietic stem cell expansion and transplantation. Experimental hematology 32, 693-695, doi:10.1016/j.exphem.2004.07.001 (2004).

48 Chotinantakul, K. \& Leeanansaksiri, W. Hematopoietic stem cell development, niches, and signaling pathways. Bone marrow research 2012, 270425, doi:10.1155/2012/270425 (2012).

49 Oh, I. H. \& Kwon, K. R. Concise review: multiple niches for hematopoietic stem cell regulations. Stem cells (Dayton, Ohio) 28, 1243-1249, doi:10.1002/stem.453 (2010).

$50 \mathrm{Li}, \mathrm{Z}$. \& Li, L. Understanding hematopoietic stem-cell microenvironments. Trends in biochemical sciences 31, 589-595, doi:10.1016/j.tibs.2006.08.001 (2006).

51 Mashiko, T. \& Yoshimura, K. How does fat survive and remodel after grafting? Clinics in plastic surgery 42, 181-190, doi:10.1016/j.cps.2014.12.008 (2015).

52 Eto, H. et al. Characterization of structure and cellular components of aspirated and excised adipose tissue. Plastic and reconstructive surgery 124, 1087-1097, doi:10.1097/PRS.0b013e3181b5a3f1 (2009).

53 Yoshimura, K. et al. Characterization of freshly isolated and cultured cells derived from the fatty and fluid portions of liposuction aspirates. Journal of cellular physiology 208, 64-76, doi:10.1002/jcp.20636 (2006).

54 Van, R. L., Bayliss, C. E. \& Roncari, D. A. Cytological and enzymological characterization of adult human adipocyte precursors in culture. The Journal of clinical investigation 58, 699704, doi:10.1172/jci108516 (1976).

55 Bjorntorp, P. et al. Isolation and characterization of cells from rat adipose tissue developing into adipocytes. Journal of lipid research 19, 316-324 (1978).

56 Halvorsen, Y. C., Wilkison, W. O. \& Gimble, J. M. Adipose-derived stromal cells--their utility and potential in bone formation. International journal of obesity and related metabolic disorders : journal of the International Association for the Study of Obesity 24 Suppl 4, S4144, doi:10.1038/sj.ijo.0801503 (2000).

57 Zuk, P. A. et al. Multilineage cells from human adipose tissue: implications for cell-based therapies. Tissue engineering 7, 211-228, doi:10.1089/107632701300062859 (2001). 
Erickson, G. R. et al. Chondrogenic potential of adipose tissue-derived stromal cells in vitro and in vivo. Biochemical and biophysical research communications 290, 763-769, doi:10.1006/bbrc.2001.6270 (2002).

Bunnell, B. A., Flaat, M., Gagliardi, C., Patel, B. \& Ripoll, C. Adipose-derived stem cells: isolation, expansion and differentiation. Methods (San Diego, Calif.) 45, 115-120, doi:10.1016/j.ymeth.2008.03.006 (2008).

Gimble, J. M., Katz, A. J. \& Bunnell, B. A. Adipose-derived stem cells for regenerative medicine. Circulation research 100, 1249-1260, doi:10.1161/01.res.0000265074.83288.09 (2007).

61 Kang, S. K. et al. Neurogenesis of Rhesus adipose stromal cells. Journal of cell science 117, 4289-4299, doi:10.1242/jcs.01264 (2004).

62 Bourin, P. et al. Stromal cells from the adipose tissue-derived stromal vascular fraction and culture expanded adipose tissue-derived stromal/stem cells: a joint statement of the International Federation for Adipose Therapeutics and Science (IFATS) and the International Society for Cellular Therapy (ISCT). Cytotherapy 15, 641-648, doi:10.1016/j.jcyt.2013.02.006 (2013).

63 Strem, B. M. et al. Multipotential differentiation of adipose tissue-derived stem cells. The Keio journal of medicine 54, 132-141, doi:10.2302/kjm.54.132 (2005).

64 D'Andrea, F. et al. Large-scale production of human adipose tissue from stem cells: a new tool for regenerative medicine and tissue banking. Tissue engineering. Part $C$, Methods 14, 233-242, doi:10.1089/ten.tec.2008.0108 (2008).

65 Tang, W. et al. White fat progenitor cells reside in the adipose vasculature. Science (New York, N.Y.) 322, 583-586, doi:10.1126/science.1156232 (2008).

66 Traktuev, D. O. et al. A population of multipotent CD34-positive adipose stromal cells share pericyte and mesenchymal surface markers, reside in a periendothelial location, and stabilize endothelial networks. Circulation research 102, 77-85, doi:10.1161/circresaha.107.159475 (2008).

67 Gimble, J. M., Bunnell, B. A. \& Guilak, F. Human adipose-derived cells: an update on the transition to clinical translation. Regenerative medicine 7, 225-235, doi:10.2217/rme.11.119 (2012).

Ortega Martinez de Victoria, E. et al. Macrophage content in subcutaneous adipose tissue: associations with adiposity, age, inflammatory markers, and whole-body insulin action in healthy Pima Indians. Diabetes 58, 385-393, doi:10.2337/db08-0536 (2009).

Dalmas, E., Clement, K. \& Guerre-Millo, M. Defining macrophage phenotype and function in adipose tissue. Trends in immunology 32, 307-314, doi:10.1016/j.it.2011.04.008 (2011).

Peer, L. A. Cell survival theory versus replacement theory. Plastic and reconstructive surgery (1946) 16, 161-168, doi:10.1097/00006534-195509000-00001 (1955).

71 Suga, H. et al. Adipose tissue remodeling under ischemia: death of adipocytes and activation of stem/progenitor cells. Plastic and reconstructive surgery 126, 1911-1923, doi:10.1097/PRS.0b013e3181f4468b (2010).

72 Eto, H. et al. The fate of adipocytes after nonvascularized fat grafting: evidence of early death and replacement of adipocytes. Plastic and reconstructive surgery 129, 1081-1092, doi:10.1097/PRS.0b013e31824a2b19 (2012).

73 Kato, H. et al. Degeneration, regeneration, and cicatrization after fat grafting: dynamic total tissue remodeling during the first 3 months. Plastic and reconstructive surgery 133, 303e313e, doi:10.1097/prs.0000000000000066 (2014).

74 Rehman, J. et al. Secretion of angiogenic and antiapoptotic factors by human adipose stromal cells. Circulation 109, 1292-1298, doi:10.1161/01.cir.0000121425.42966.f1 (2004).

75 Planat-Benard, V. et al. Plasticity of human adipose lineage cells toward endothelial cells: physiological and therapeutic perspectives. Circulation 109, 656-663, doi:10.1161/01.cir.0000114522.38265.61 (2004). 
76 Miranville, A. et al. Improvement of postnatal neovascularization by human adipose tissuederived stem cells. Circulation 110, 349-355, doi:10.1161/01.cir.0000135466.16823.d0 (2004).

77 Sumi, M. et al. Transplantation of adipose stromal cells, but not mature adipocytes, augments ischemia-induced angiogenesis. Life sciences 80, 559-565, doi:10.1016/j.Ifs.2006.10.020 (2007).

78 Nakagami, H. et al. Novel autologous cell therapy in ischemic limb disease through growth factor secretion by cultured adipose tissue-derived stromal cells. Arteriosclerosis, thrombosis, and vascular biology 25, 2542-2547, doi:10.1161/01.ATV.0000190701.92007.6d (2005).

79 Cai, L. et al. Suppression of hepatocyte growth factor production impairs the ability of adipose-derived stem cells to promote ischemic tissue revascularization. Stem cells (Dayton, Ohio) 25, 3234-3243, doi:10.1634/stemcells.2007-0388 (2007).

80 Matsumoto, D. et al. Cell-assisted lipotransfer: supportive use of human adipose-derived cells for soft tissue augmentation with lipoinjection. Tissue engineering 12, 3375-3382, doi:10.1089/ten.2006.12.3375 (2006).

81 Jeong, J. H. Adipose stem cells and skin repair. Current stem cell research \& therapy 5, 137140, doi:10.2174/157488810791268690 (2010).

82 Suga, H., Glotzbach, J. P., Sorkin, M., Longaker, M. T. \& Gurtner, G. C. Paracrine mechanism of angiogenesis in adipose-derived stem cell transplantation. Annals of plastic surgery 72, 234241, doi:10.1097/SAP.0b013e318264fd6a (2014).

83 Lovett, M., Lee, K., Edwards, A. \& Kaplan, D. L. Vascularization strategies for tissue engineering. Tissue engineering. Part $B$, Reviews 15, 353-370, doi:10.1089/ten.TEB.2009.0085 (2009).

84 Zhang, K. et al. Strategy for constructing vascularized adipose units in poly(l-glutamic acid) hydrogel porous scaffold through inducing in-situ formation of ASCs spheroids. Acta biomaterialia 51, 246-257, doi:10.1016/j.actbio.2017.01.043 (2017).

85 Miyamoto, Y., Ikeuchi, M., Noguchi, H., Yagi, T. \& Hayashi, S. Enhanced Adipogenic Differentiation of Human Adipose-Derived Stem Cells in an In Vitro Microenvironment: The Preparation of Adipose-Like Microtissues Using a Three-Dimensional Culture. Cell medicine 9, 35-44, doi:10.3727/215517916x693096 (2017).

86 Dazzi, F. \& Krampera, M. Mesenchymal stem cells and autoimmune diseases. Best practice \& research. Clinical haematology 24, 49-57, doi:10.1016/j.beha.2011.01.002 (2011).

$87 \mathrm{Li}, \mathrm{W}$. et al. Mesenchymal stem cells: a double-edged sword in regulating immune responses. Cell death and differentiation 19, 1505-1513, doi:10.1038/cdd.2012.26 (2012).

88 Gazdic, M., Volarevic, V., Arsenijevic, N. \& Stojkovic, M. Mesenchymal stem cells: a friend or foe in immune-mediated diseases. Stem cell reviews and reports 11, 280-287, doi:10.1007/s12015-014-9583-3 (2015).

89 Raffaghello, L. et al. Human mesenchymal stem cells inhibit neutrophil apoptosis: a model for neutrophil preservation in the bone marrow niche. Stem cells (Dayton, Ohio) 26, 151-162, doi:10.1634/stemcells.2007-0416 (2008).

90 Cassatella, M. A. et al. Toll-like receptor-3-activated human mesenchymal stromal cells significantly prolong the survival and function of neutrophils. Stem cells (Dayton, Ohio) 29, 1001-1011, doi:10.1002/stem.651 (2011).

91 Brandau, S. et al. Tissue-resident mesenchymal stem cells attract peripheral blood neutrophils and enhance their inflammatory activity in response to microbial challenge. Journal of leukocyte biology 88, 1005-1015, doi:10.1189/jlb.0410207 (2010).

92 Nemeth, K. et al. Bone marrow stromal cells attenuate sepsis via prostaglandin $\mathrm{E}(2)$ dependent reprogramming of host macrophages to increase their interleukin-10 production. Nature medicine 15, 42-49, doi:10.1038/nm.1905 (2009).

93 Bernardo, M. E. \& Fibbe, W. E. Mesenchymal stromal cells: sensors and switchers of inflammation. Cell stem cell 13, 392-402, doi:10.1016/j.stem.2013.09.006 (2013). 
94 Balint, R., Cassidy, N. J. \& Cartmell, S. H. Electrical stimulation: a novel tool for tissue engineering. Tissue engineering. Part B, Reviews 19, 48-57, doi:10.1089/ten.TEB.2012.0183 (2013).

95 Nuccitelli, R. Endogenous ionic currents and DC electric fields in multicellular animal tissues. Bioelectromagnetics Suppl 1, 147-157, doi:10.1002/bem.2250130714 (1992).

96 Zhao, M. Electrical fields in wound healing-An overriding signal that directs cell migration. Seminars in cell \& developmental biology 20, 674-682, doi:10.1016/j.semcdb.2008.12.009 (2009).

97 McCaig, C. D., Rajnicek, A. M., Song, B. \& Zhao, M. Controlling cell behavior electrically: current views and future potential. Physiological reviews 85, 943-978, doi:10.1152/physrev.00020.2004 (2005).

98 Farboud, B., Nuccitelli, R., Schwab, I. R. \& Isseroff, R. R. DC electric fields induce rapid directional migration in cultured human corneal epithelial cells. Experimental eye research 70, 667-673, doi:10.1006/exer.2000.0830 (2000).

99 Song, B., Zhao, M., Forrester, J. V. \& McCaig, C. D. Electrical cues regulate the orientation and frequency of cell division and the rate of wound healing in vivo. Proceedings of the National Academy of Sciences of the United States of America 99, 13577-13582, doi:10.1073/pnas.202235299 (2002).

100 Nuccitelli, R. A role for endogenous electric fields in wound healing. Current topics in developmental biology 58, 1-26, doi:10.1016/s0070-2153(03)58001-2 (2003).

101 Titushkin, I. \& Cho, M. Regulation of cell cytoskeleton and membrane mechanics by electric field: role of linker proteins. Biophysical journal 96, 717-728, doi:10.1016/j.bpj.2008.09.035 (2009).

102 Lin, F. et al. Lymphocyte electrotaxis in vitro and in vivo. Journal of immunology (Baltimore, Md. : 1950) 181, 2465-2471, doi:10.4049/jimmunol.181.4.2465 (2008).

103 Ud-Din, S. et al. Angiogenesis is induced and wound size is reduced by electrical stimulation in an acute wound healing model in human skin. PloS one 10, e0124502, doi:10.1371/journal.pone.0124502 (2015).

104 Poh, P. S. P. et al. Osteogenic Effect and Cell Signaling Activation of Extremely Low-Frequency Pulsed Electromagnetic Fields in Adipose-Derived Mesenchymal Stromal Cells. Stem cells international 2018, 5402853, doi:10.1155/2018/5402853 (2018).

105 Kanno, S. et al. Establishment of a simple and practical procedure applicable to therapeutic angiogenesis. Circulation 99, 2682-2687, doi:10.1161/01.cir.99.20.2682 (1999).

106 Sheikh, I. et al. Effect of electrical stimulation on arteriogenesis and angiogenesis after bilateral femoral artery excision in the rabbit hind-limb ischemia model. Vascular and endovascular surgery 39, 257-265, doi:10.1177/153857440503900307 (2005).

107 Bai, H., Forrester, J. V. \& Zhao, M. DC electric stimulation upregulates angiogenic factors in endothelial cells through activation of VEGF receptors. Cytokine 55, 110-115, doi:10.1016/j.cyto.2011.03.003 (2011).

$108 \mathrm{Kim}, \mathrm{l}$. S. et al. Novel effect of biphasic electric current on in vitro osteogenesis and cytokine production in human mesenchymal stromal cells. Tissue engineering. Part A 15, 2411-2422, doi:10.1089/ten.tea.2008.0554 (2009).

109 Tandon, N. et al. Alignment and elongation of human adipose-derived stem cells in response to direct-current electrical stimulation. Conference proceedings : ... Annual International Conference of the IEEE Engineering in Medicine and Biology Society. IEEE Engineering in Medicine and Biology Society. Annual Conference 2009, 6517-6521, doi:10.1109/iembs.2009.5333142 (2009). 


\section{CHAPTER 2}

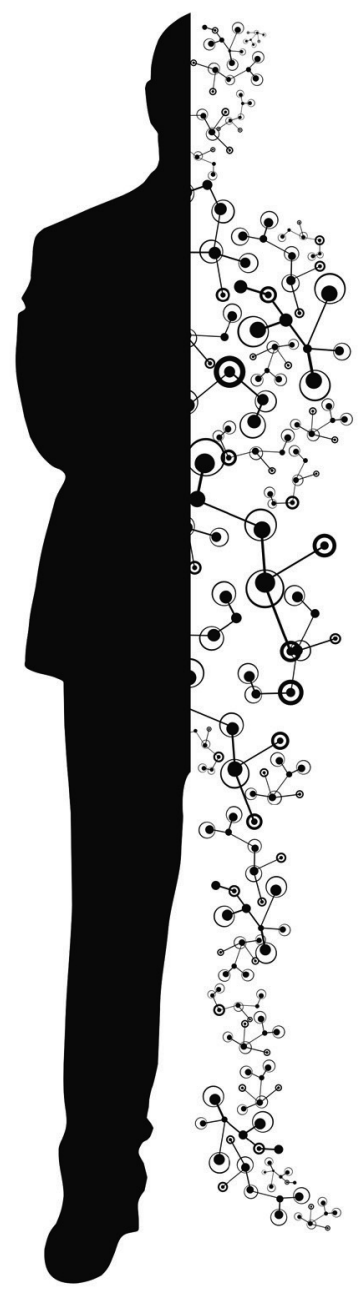




\section{2}

\section{Efficacy and Safety of Autologous Fat Transfer in Facial Reconstructive}

Surgery: A Systematic Review and Meta-Analysis.

Krastev TK, Beugels J, Hommes J, Piatkowski A, Mathijssen I, van der Hulst R 
ABSTRACT

\section{Importance:}

The use of autologous fat transfer (AFT) or lipofilling for correcting contour deformities is seen as one of the major breakthroughs in reconstructive plastic surgery. Its applications in facial reconstructive surgery have been of particular interest owing to the prospect of achieving autologous reconstruction by a minimally invasive approach. However, its unpredictability and variable degree of resorption have limited its utility and much scepticism still exists regarding its efficacy. Furthermore, more than 2 decades of clinical research have produced a highly fragmented body of evidence that has not been able to provide definite answers.

\section{Objective:}

To investigate the safety and efficacy of AFT in facial reconstruction through a systematic review and meta-analysis.

\section{Data sources:}

A literature search was performed in PubMed, Embase, and the Cochrane Library from inception to October 11, 2017.

\section{Study selection:}

All published studies investigating the efficacy and safety of AFT in facial reconstructive surgery.

\section{Data extraction and synthesis:}

Two independent reviewers performed data extraction systematically, adhering to the PRISMA guidelines. Summary measures were pooled in a random-effects model metaanalysis.

\section{Main outcomes and measures:}

The patient and surgeon satisfaction, graft survival, number of AFT sessions, and the incidence of AFT-related complications were the main outcomes of interest in this metaanalysis. 


\section{Results:}

This systematic review resulted in the inclusion 52 relevant studies consisting of 1568 unique patients. These included 4 randomized clinical trials, 11 cohort studies, and 37 case series. The overall follow-up averaged 1.3 years after AFT. Meta-analysis revealed a very high overall patient satisfaction rate of $91.1 \%(95 \% \mathrm{Cl}, 85.1 \%-94.8 \%)$ and overall surgeon satisfaction rate of $88.6 \%(95 \% \mathrm{Cl}, 83.4 \%-92.4 \%)$. The number of AFT sessions required to achieve the desired result was $1.5(95 \% \mathrm{Cl}, 1.3-1.7)$ and $50 \%$ to $60 \%$ of the injected volume was retained at 1 year. Only $4.8 \%(95 \% \mathrm{Cl}, 3.3 \%-6.9 \%)$ of procedures resulted in clinical complications.

\section{Conclusions and relevance:}

To our knowledge, this study provides the first overview of the current knowledge about AFT in facial reconstructive surgery. Our results confirm that AFT is an effective technique for treating soft-tissue deformities in the head and neck, with low rate of minor complications.

\section{KEY POINTS}

Question: Is autologous fat transfer (lipofilling) an effective treatment for soft tissue deformities in the face?

Findings: This meta-analysis of 52 studies found AFT to be associated with a high patient (91\%) and surgeon (89\%) satisfaction after 1.5 AFT sessions. Volume retention stabilized at $50-60 \%$ at one year only $5 \%$ of procedures resulted in clinical complications.

Meaning: This thorough analysis of the combined data from all published studies confirms that AFT is a safe and effective treatment in facial reconstructive surgery.

\subsection{Introduction}

Autologous fat transfer (AFT), also known as lipofilling, is regarded as an elegant technique that excels in correcting soft-tissue contour deformities and volume deficits in almost any body part. Its application in the face has always been of particular interest as it allows for precise titration and injection of patient's own fat by means of a minimally invasive approach. AFT can play an important role in the treatment of disfiguring congenital 
syndromes such as progressive hemifacial atrophy (Parry-Romberg Syndrome) or hemifacial (craniofacial) microsomia. For these disorders, the only viable alternative for correcting volume deficits remains the free flap transfer, which often leads to disappointing results in the long term due to sagging and disruption of facial musculature. Another disorder that can greatly benefit from AFT is HIV-associated lipodystrophy, where antiretroviral therapy can lead to devastating facial wasting. The prospect of restoring facial volume without relying on allogenic fillers of implants makes AFT a promising solution in these patients. Due to these properties, fat transfer has also been increasingly applied in the treatment of acquired softtissue defects in the face such as adhesive scars and even burns (Figure 1).
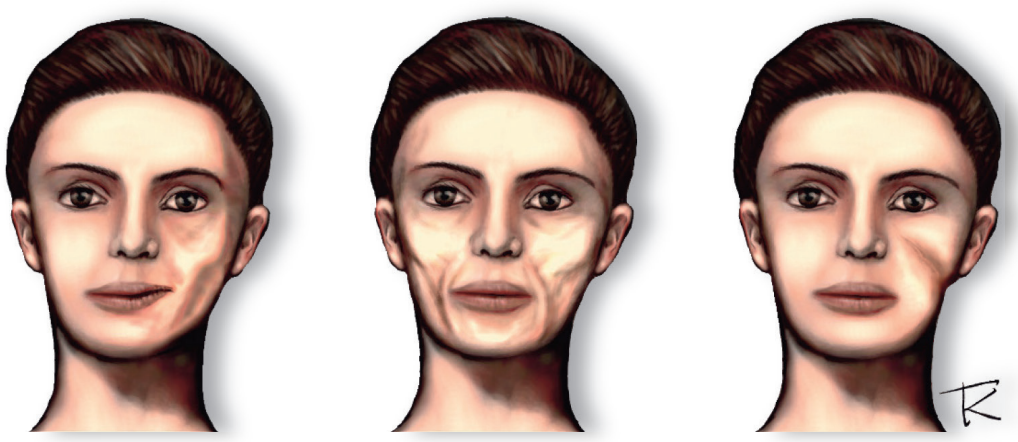

Figure 1: Indications of AFT in the face: A) Parry-Romberg Syndrome, B) HIV-associated lipodystrophy, C) Acquired facial scar deformity.

However, the unpredictability of long-term graft retention has hampered the widespread acceptance and application of AFT in reconstructive plastic surgery. The loss of a significant portion of the grafted volume seems to be an inherent limitation with AFT, which has been the main criticism with this technique. This has engendered divergent and even polarized opinions among plastic surgeons worldwide with respect to whether AFT offers a reliable solution for correcting soft-tissue deformities in facial reconstructive surgery. Unfortunately, more than two decades of clinical research have failed to provide convincing evidence on this matter. The reason is that published literature comprises of a large number of small studies investigating a diversity of outcome measures in different patient populations, producing a body of evidence that is highly fragmented and difficult to analyze. 
This has inspired us to perform the first systematic review and meta-analysis on the subject, aimed to facilitate interpretation of the evidence on the efficacy and safety of AFT in facial reconstructive surgery by clinicians, guideline committees and policy-makers.

\subsection{Patients and methods}

This systematic review adhered to the standards of the Cochrane Handbook of Systematic Reviews of Interventions ${ }^{1}$ and the Meta-analysis Of Observational Studies in Epidemiology $(\mathrm{MOOSE})^{2}$ and was written in the format provided by the Preferred Reporting Items of Systematic Reviews and Meta-Analyses (PRISMA) statement ${ }^{3}$ (eTable 6 - Online supplement). A comprehensive, reproducible electronic search was conducted in PubMed, EMBASE and the Cochrane Library to identify all published studies with human subjects receiving the intervention autologous fat transfer for facial reconstruction (eTable 1,2 - Online supplement). The search was performed on October $11^{\text {th }} 2017$. Eligibility assessment was performed in a standardized manner. The retrieved hits were screened and reviewed by two independent reviewers (T.K and J.B.) based on the title and abstract using predefined inclusion and exclusion criteria. Disagreements were solved through discussion until consensus was reached. An additional assessment was performed based on the full-text versions of all selected papers and those with insufficient information in the title and abstract. All references were stored in Endnote Reference Management Tool, (version X7.3.1, Thomson Reuters).

To be able to conduct a meta-analysis, a thorough extraction of all relevant data was performed (eTable 3 - Online supplement). In some occasions, authors were contacted to send additional data. In cases when key data was reported only in graphs or figures, it was extracted using the WebPlotDigitizer software ${ }^{4}$. Whenever necessary, units were transformed to a standard format to ensure comparability and allow pooling of data. Continuous variables reported in the form of median \pm range were transformed into mean \pm standard deviations (SD) using the standard estimating equations used for meta-analyses ${ }^{5}$. Custom outcome measurement scales and scores were also standardized accordingly. Finally, if studies from the same author or institution conducted in the same time period and reporting the same outcomes were suspected for overlap of more than $25 \%$ of the sample size, only the largest or most relevant study was included. Meta-analysis was performed using the "metafor" package ${ }^{6}$ of the R-software ${ }^{7}$. Summary measures were pooled in a 
standard random-effects model and presented as forest plots. Heterogeneity was assessed using the $\mathrm{I}^{2}$-statistic, which was tolerable if below $40 \%$. Publication bias considered acceptable if upon visual inspection, the distribution of studies was approximately symmetrical.

\subsection{Results}

\subsubsection{Study selection}

The electronic search yielded a total of 370 unique hits (eFigure 1- Online supplement). Screening of the title and abstract lead to the inclusion of 102 studies for further evaluation. A total of 43 clinical trials were selected through further screening of the full-text. Reasons for exclusion consisted of the unavailability of the full-text, secondary sources (reviews, commentaries), the use of AFT for cosmetic indications, enhancement with supplements (such as platelet rich plasma, PRP or stromal-vascular fraction, SVF). Case reports (defined as reports of five cases or less) were also not considered for inclusion. Screening of the citations, references and related articles of the 43 selected trials yielded nine additional hits. It should be noted that some cohort studies consisting of one or more treatment arms, which did not meet the inclusion criteria of the predefined PICO (such as use of supplements or combination treatments), were not included in the analysis. This was also the case whenever outcomes of interest were only relevant or measured in one of the treatment groups.

\subsection{Study characteristics}

Fifty-two clinical trials were identified for inclusion ${ }^{8-59}$ (eTable 4). They consisted of 37 caseseries, eleven cohort studies and four randomized-controlled trials. The majority of these had a retrospective design $(n=44)$ and all studies were conducted between 1986 and 2016 .

\subsubsection{Population}

From the 52 included studies (1,549 patients), only one study was suspected of significant overlap in patient populations ${ }^{45}$, resulting in 51 studies with a total of 1,533 unique patients. Most commonly, a study would include patients of a specific diagnosis. In fourteen studies $11,12,17,19,20,23,33,35,37,38,44,45,49,54$, it involved patients with HIV-associated lipodystrophy, comprising of 650 patients, thus representing the most common indication for which AFT is 
described in published literature. The majority (76.9\%) were males and the mean age at the time of the first AFT session was 42.6 years $(95 \% \mathrm{Cl} 34.4-46.7)$. Furthermore, sixteen studies $10,14-16,25,26,28,32,43,47,50,52,53,57-59$ consisting of 409 patients evaluated AFT in patients with congenital disorders. From this group, 201 (49.1\%) patients suffered from Parry-Romberg syndrome, 181 (44.3\%) from craniofacial microsomia and the remaining 27 patients (6.6\%) represent a mixed group of craniofacial microsomia, Goldenhar syndrome and Treacher Collins from a single study ${ }^{32}$. The mean age at the time of treatment with AFT was 23.8 years $(95 \% \mathrm{Cl} 19.8-27.8)$ in this group. Another seventeen studies $8,9,13,18,21,22,24,27,29,30,39-41,46,48,55,56$ formed a more heterogeneous group consisting 326 patients with a mean age of 36.8 years $(95 \% \mathrm{Cl} 31.5-42.1)$ treated with AFT for a variety of acquired facial deformities such. In seven studies it was applied for treating small contour deformities such as facial scars (e.g. traumatic, thermal injury) and in another two AFT was performed for lip contouring after resection of epithelial cancer or cleft-lip surgery. Five studies involved the restoration of contour deformities after maxillofacial reconstruction after trauma and two - after autologous reconstruction of irradiated head and heck cancer. Finally, one study performed facial contouring with AFT in addition to conventional surgical treatment for facial burns involving large areas of the face.

Each of the remaining four studies ${ }^{31,34,42,51}$ (148 patients) involved a mixed group of patients from any of the abovementioned indications.

\subsubsection{Intervention}

From the included trials, the vast majority adhered to the principles of AFT as described by Coleman $^{60}$, with the occasional minor variation in the surgical instruments used.

\subsubsection{Control group}

Only sixteen studies examining AFT included a control group, which most often involved patients receiving AFT with supplements $(n=9)$ such as $\mathrm{PRP}^{19,21}, \mathrm{SVF}^{14,21,51,52,59}$, bone-marrow derived mesenchymal stem-cells ${ }^{26}$ or cultured adipose-derived stem cells ${ }^{28}$. As the scope of this paper is limited to the use of AFT without supplements, treatment arms that involved enhancement of AFT with the abovementioned supplements were excluded from the analysis. Another four studies involved additional treatment arms receiving (semi-) permanent fillers ${ }^{36-38,44}$, two studies compared AFT to autologous flaps ${ }^{47,53}$ and the last study - with dermis-fat grafts ${ }^{33}$. 


\subsubsection{Outcome measures}

\subsubsection{Patient satisfaction:}

The satisfaction of the patient with respect to the result of the AFT reconstruction was measured in 30 trials. Twelve of these studies presented the result using categorical or "Likert scores" and reported the number of patients with a certain score (category) at the end of the treatment. The Likert scales consisted of a scale with two to five categories corresponding to degrees of satisfaction with the outcome (e.g. very dissatisfied, dissatisfied, satisfied, very satisfied) or opinion about the rate of improvement (e.g. very poor, poor, fair, good, excellent). To standardize the scales, scores were dichotomized into two distinct categories - satisfied versus dissatisfied. For all studies, such a cut-off point between the positive and negative categories was easily identifiable. Furthermore, 22 studies presented satisfaction scores in the form of custom-made visual analogue scales (VAS), ranging from 4 to 50 levels, which were transformed into a standard 10-point VAS scale ( $0=$ maximum dissatisfaction, $10=$ maximum satisfaction).

\subsubsection{Surgeon satisfaction/evaluation:}

Twenty-nine studies reported the surgeon's satisfaction, which typically consisted of visual evaluation of pre- and postoperative photographs to determine the rate of success of the AFT treatment. Sixteen studies reported surgeon's evaluation using Likert scales with two to five categories describing the effect of AFT on the clinical result, which were subsequently dichotomized into good versus poor. Ten studies reported mean VAS scores (range 0-3 to 020) and these were also transformed to a standard 0-10 scale ( $0=$ very poor result, $10=$ excellent result).

\subsubsection{Volume measurements:}

From sixteen studies addressing fat resorption through volume measurements, three of them attempted to estimate volume changes by evaluating tissue thickness using ultrasound or CT-scan and were excluded from the analysis ${ }^{8,16,48}$. The remaining thirteen studies that utilized appropriate imaging techniques such as 3D image capture devices $25,28,36,50,53,58$, or Computed Tomography (CT) 14,19,20,43,52. Eight of these investigated volume retention in exclusively congenital patients (Parry-Romberg and craniofacial microsomia), while the remaining five involved HIV-associated lipodystrophy patients. The percentage graft 
retention represents the fraction of the measured gain in volume, relative to the total volume of injected fat, as shown in the following formula:

Graft retention $\%=\frac{\text { postop. vol. }- \text { preop.vol. }}{\text { total injected volume }}=\frac{\text { vol.gained }}{\text { vol. injected }} \times 100 \%$ I

The relationship between the graft retention with time was investigated by means of a meta-regression.

\subsubsection{AFT-related complications:}

Forty-five studies evaluated the safety of AFT in terms of immediate and late AFT-related complications. A total of 65 complications were documented in 1,755 AFT procedures (3.7\%), with the majority consisting of irregularities or asymmetry post-AFT (chiefly in HIVpatients). Only two infections (0.1\%), two cases of fat necrosis $(0.1 \%)$ and ten hematomas $(0.6 \%)$ were reported and these represent the only true complications encountered in this vast cohort. Additional meta-analysis was performed to derive the proportion of complications per AFT procedure.

\subsubsection{Time frame}

The average clinical follow-up after AFT was $1.3(95 \% \mathrm{Cl} 0.9-1.6)$ years.

\subsection{Meta-analysis results}

Results of individual studies are presented in eTable 4. A random-effects model metaanalysis was performed with pooled data of each outcome. Categorical data was dichotomized and presented as a meta-analysis of proportions, which can be treated as percentages.

\subsubsection{Patient satisfaction}

Meta-analysis of categorical data revealed an overall proportion of satisfied patients of 0.91 (95\% Cl 0.85-0.95), after a mean follow-up of 2.0 years (Figure 2). It was highest in studies with mixed patients (96\%), followed by HIV-lipodystrophy (94\%), congenital deformities (92\%) and lowest in studies investigating patients with acquired deformities (82\%). Metaanalysis of reported VAS-scores resulted in an average score of 7.9 (95\% Cl 7.5-8.3), with little variation between the mean scores of individual studies or subgroups (Figure 3). 


\subsubsection{Surgeon satisfaction/evaluation}

As with patient satisfaction, a very high proportion of the plastic surgeons were satisfied with the result $-0.89(95 \% \mathrm{Cl}$ 0.83-0.92) after a mean follow-up of 1.2 years (Figure 2). The highest overall satisfaction rate was noted in congenital deformities (95\%), followed by HIVlipodystrophy (90\%) and acquired deformities (87\%). The mixed patients displayed the lowest surgeon satisfaction rate (78\%), though this subgroup consisted of a single study with only 27 patients. Subsequent meta-analysis of VAS-scores led to an overall score of 7.8 (95\% $\mathrm{Cl} 7.3-8.4)$ and similarly to the scores reported by patients, surgeon scores varied little between relevant subgroups (Figure 3).

\subsubsection{Volume measurements}

Twelve studies provided data on volume retention, which was plotted against time in a meta-regression model. Patients with congenital deformities displayed a trend toward gradual decrease, stabilizing at $59 \%(95 \% \mathrm{Cl} 45-72)$ after one year of follow-up (Figure $4 a)$. On the contrary, conflicting patterns of volume changes were seen in HIV-associated lipodystrophy patients, where volume retention decreased to $51 \%(95 \% \mathrm{Cl} 37-66)$ in some studies and or increased drastically to $238 \%(95 \% \mathrm{Cl} 201-274)$ in others (see Figure $4 b)$.

\subsubsection{Number of AFT sessions}

Meta-analysis of pooled data from 50 studies (1,525 patients) showed that a mean of 1.5 sessions $(95 \% \mathrm{Cl} 1.3-1.7)$ were needed to achieve the desired result and on average $27 \mathrm{ml}$ of fat was injected per procedure (eFigure 5 - Online supplement). Subgroup analysis revealed some variation between indications with the highest number of procedures being performed for the correction of congenital deformities (1.8 sessions) and the lowest in HIVassociated facial lipodystrophy (1.1 sessions).

\subsubsection{Complications}

The incidence of AFT-related clinical complications based on our meta-analysis was low $0.05(95 \% \mathrm{Cl} 0.03-0.07)$, or equivalent to $5 \%$ of the AFT procedures (eFigure 6 - Online supplement). Only in HIV-associated lipodystrophy patients, a higher rate of complications was reported (7\%), however these were not commonly encountered AFT-related complications as in other indications but consisted mainly of asymmetry or overcorrection due to lipohypertrophy. 


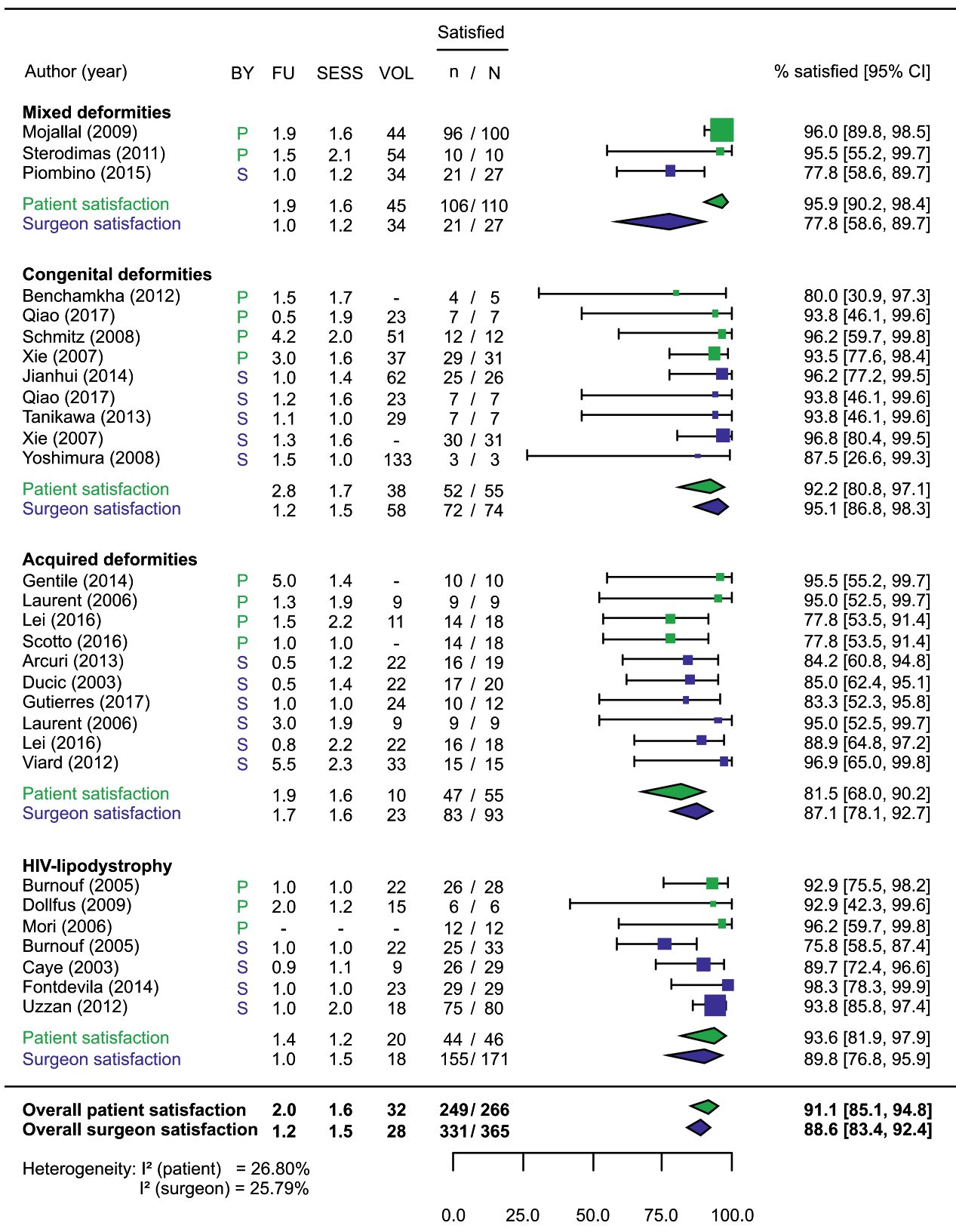

Percentage satisfied patients/surgeons - Likert scales

Figure 2: Patient and surgeon satisfaction (categorical data). Legend: AFT=autologous fat transfer, $P=$ patient,$S=$ surgeon. 


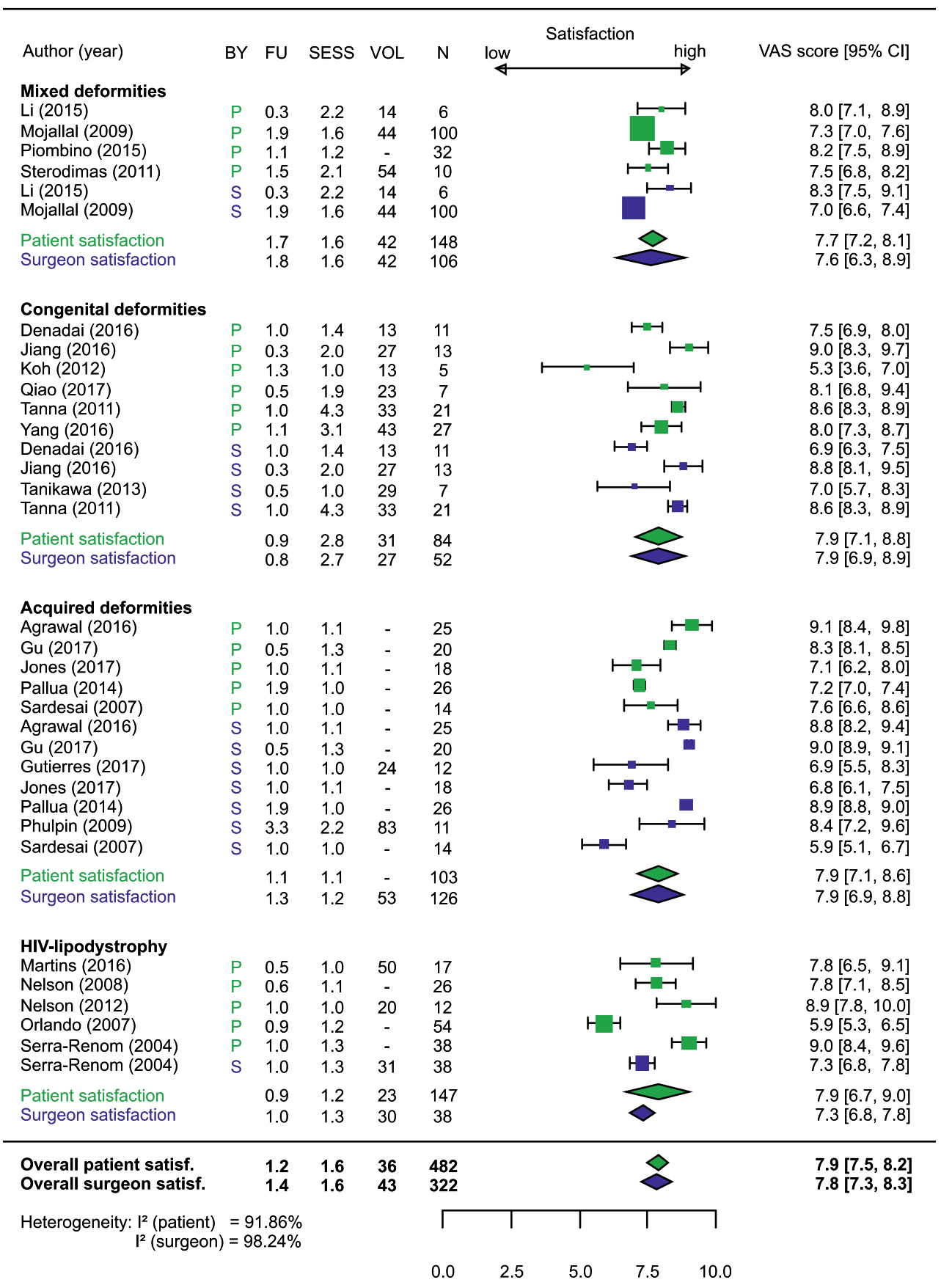

Satisfaction score of patients/surgeons - VAS scales

Figure 3: Patient and surgeon satisfaction (continuous data). Legend: AFT=autologous fat transfer, $P=$ patient, $S=$ surgeon. 

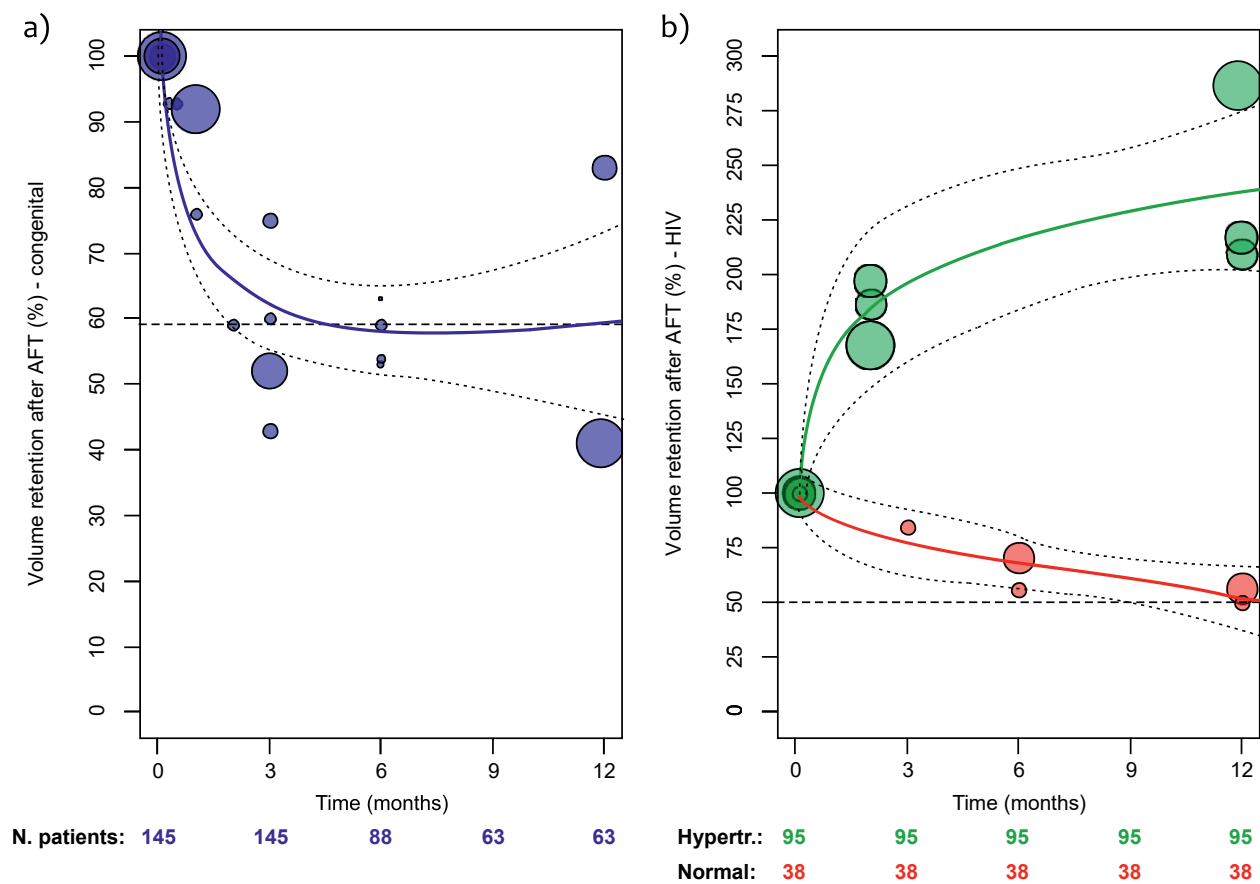

Figure 4: Meta-regression of graft retention over time for patients who underwent facial reconstruction (A) Volume retention in congenital patients (blue circles). (B) Volume retention in HIVassociated lipodystrophy patients. Studies were categorised based on whether patients experienced a normal (expected) rate of resorption (red circles) or extreme hypertrophy (green circles). Legend: size of the circles corresponds to sample size, thick lines represent meta-regression effect estimates and their respective $95 \% \mathrm{Cl}$ (gray dotted lines). The total number of patients with volume measurements at each time-point in the follow-up is shown below (N. patients). Legend: HIV= human immunodeficiency virus, Hyperfr. =fat hypertrophy group, Normal=normal resorption pattern group.

\subsection{Discussion}

This meta-analysis aims to provide a clinically relevant, quantifiable measure on the overall efficacy of autologous fat transfer in facial reconstructive surgery. Our results demonstrate a clear trend toward a very high rate of patient (91\%) and surgeon (89\%) satisfaction after a mean of 1.5 procedures and a low rate (5\%) of minor complications. The volume retention in patients with congenital facial deformities showed a wide variation between studies, ranging between $40 \%$ to over $80 \%$ with pooled effect estimate of $59 \%$ at one year. In HIVlipodystrophy patients, deviations were even more extreme and two distinct patterns of volume changes were observed. The former resembled the resorption pattern of congenital 
patients, gradually decreasing to $51 \%$ at one year. The latter, on the other hand, led to a spontaneous increase in volume to more than $238 \%$ at one year. This could, in theory, be attributed to extreme lipohypertrophy of grafted fat, which has been commonly reported as an adverse event in studies with HIV-patients. Whether this effect is the result of the choice of a donor site affected by lipohypertrophy (cervical hump, abdomen or breast area), metabolic (medication) or iatrogenic stimuli, must be evaluated in future studies.

By a thorough examination of the totality of the evidence, it can be deduced that AFT seems to be an effective procedure that leaves the vast majority of patients and surgeons satisfied with the result after about a year of clinical follow-up. It is clear that future studies will play a crucial role in providing higher quality evidence that would allow for more nuanced recommendations regarding specific subgroups or indications, evaluating factors influencing the (long-term) volume retention, as well as the added value of supplements. As setting up randomized-controlled trials (RCTs) in some of these areas can be difficult, they should be evaluated whenever possible by prospective study design with the use of control groups and validated outcome measurement instruments such as 3D volume assessments and quality of life scales (See Table 5 - Online supplement).

\subsection{Limitations}

The main limitation of this meta-analysis as well as the accumulated body of knowledge on AFT in general, remains the low level-of-evidence of published studies. Unlike the widely accepted approach, where the adoption of a new therapy is based on the achievement of statistical significance for the primary outcome from well-executed RCTs or large cohorts, this has not been possible in the case of AFT. Its unique properties that give it competitive edge above conventional techniques, have also robbed it of an alternative treatment to perform in a control group. Setting up randomized trials with AFT has therefore been difficult due to practical and ethical challenges. Non-randomized cohorts, on the other hand could potentially introduce bias by allocating the treatment based on the indication, severity or the patient/surgeon's preference. Inevitably, this would produce groups that are not comparable, thereby reducing the methodological quality of the study. Nevertheless, studies involving control groups not treated with AFT are scarce, and the few such trials that have been conducted only compare different AFT techniques (with or without supplements) with each other. More than two decades after the popularization of AFT by Coleman ${ }^{60}$, clinical 
research on the topic has not been successful in providing convincing evidence on its efficacy and safety.

Another important obstacle that hinders progress in the field of autologous fat transfer, is the lack of practical and reliable instruments to quantify the absolute effect of treatment. For many years, plastic surgeons have relied on non-validated, custom-made questionnaires to measure patient satisfaction, routinely use pre- and postoperative photographs for a slightly more objective confirmation of the findings. These outcomes have been reported as either continuous (means $\pm S D$ ) or categorical (frequencies) variables, based on the type of questionnaire or scale. Each of these presents with its own advantages and limitations. As most retrospective studies rarely measured outcomes preoperatively, determining of the absolute effect of AFT becomes difficult in the absence of baseline values. In addition, the use of a continuous scale is of limited value when attempting to quantify the effect of AFT with different baseline scores. It is arguable that the same in change in different satisfaction scores can have different clinical significance. For example, an increase in a satisfaction from 1- 4 out of 10 can be classified as an unsatisfactory result, while the same increase in another patient from a score of $4-7$ out of 10 would be seen as successful. When dealing with studies reporting of nominal (categorical) data, outcomes such as the rate of satisfaction are often reported as frequencies of patients falling in each category (e.g. dissatisfied, satisfied, very satisfied). As such an approach does not rely on baseline values, it can more accurately reflect the absolute effect of AFT, though it lacks the precision of continuous data.

For the abovementioned reasons, performing a meta-analysis on this topic is highly challenging as the paucity of relevant control groups and pre- versus postoperative data prevents applying widely accepted statistical measures of effect such as the relative risk or odds ratios. Therefore, this meta-analysis focused on providing pooled effect estimates that indicate the size, direction and confidence intervals for each outcome.

Dealing with heterogeneity is another major challenge with meta-analyses. Pooling a large number of studies with potentially different patient demographics, indications and outcome measurements can result in important heterogeneity that could reduce the validity of the effect estimates. To correct for this, a random-effects model was applied to all cases and whenever possible, subgroup meta-analyses were performed by pooling studies with similar indications together. It is important to note that despite the potential differences between 
included studies, the pooled effect estimates demonstrate a clear convergence toward a very high rate of satisfaction with the procedure and a low rate of clinical complications. As the indications of AFT in facial reconstructive surgery consists of a heterogeneous group of indications and patients, it can be argued that such a general approach delivers evidence on precisely the population this meta-analysis aims to draw conclusions about.

\subsection{Conclusion}

Autologous fat transfer seems to be an effective treatment for contour deformities in facial reconstruction, as reflected by the high patient satisfaction (91\%) and surgeon satisfaction $(89 \%)$, achieved after a mean of 1.5 procedures. Volume retention was observed to decrease to $50-60 \%$ after one year but needs further investigation, especially in HIV-associated facial lipodystrophy patients. Autologous fat transfer can be considered a safe treatment, with only $5 \%$ of procedures resulting in minor complications.

\section{$1.9 \quad$ Funding}

This research was funded by the Dutch Society of Plastic Surgeons for the purpose of Autologous Fat Transfer guideline development in the Netherlands.

\subsection{Conflict of interests}

None of the authors has a financial interest in any of the products, devices, or drugs mentioned in this manuscript. 


\subsection{References}

1. Higgins JPT GSeCHfSRoIVuMTCC, 2011. Available from www.handbook.cochrane.org.

2. Stroup DF, Berlin JA, Morton SC, et al. Metaanalysis of observational studies in epidemiology: a proposal for reporting. Meta-analysis of Observational Studies in Epidemiology (MOOSE) group. JAMA. 2000;283(15):2008-2012.

3. Knobloch K, Yoon U, Vogt PM. Preferred reporting items for systematic reviews and meta-analyses (PRISMA) statement and publication bias. $J$ Craniomaxillofac Surg. 2011;39(2):91-92.

4. WebPlotDigitizer software. Available at: http://arohatgi.info/WebPlotDigitizer. Accessed November 15 .

5. Hozo SP, Djulbegovic B, Hozo I. Estimating the mean and variance from the median, range, and the size of a sample. BMC Med Res Methodol. 2005;5:13.

6. Viechtbauer W. Conducting meta-analyses in $\mathrm{R}$ with the metafor package. Journal of Statistical Software. 2010;36(3):1-48.

7. R Core Team (2016). R: A language and environment for statistical computing. $R$ Foundation for Statistical Computing V, Austria. URL, https://www.R-project.org/.

8. Agrawal KS, Bachhav M, Naik CS, Tanwar H, Sankhe SS. Autologous Fat Transfer for Esthetic Contouring of Face in Posttraumatic Nonfunctional Maxillofacial Deformities. Craniomaxillofac Trauma Reconstr. 2016;9(2):113-120.

9. Arcuri F, Brucoli M, Baragiotta N, Stellin L, Giarda $M$, Benech $A$. The role of fat grafting in the treatment of posttraumatic maxillofacial deformities. Craniomaxillofacial Trauma and Reconstruction. 2013;6(2):121-126.

10. Benchamkha Y, Ettalbi S, Droussi H, Bahechar N, Boukind EH. [Lipostructure(R) for morphologic restauration in Parry-Romberg syndrome: about 12 cases]. Ann Chir Plast Esthet. 2012;57(3):273-280.

11. Burnouf $M$, Buffet $M$, Schwarzinger $M$, et al. Evaluation of Coleman lipostructure for treatment of facial lipoatrophy in patients with human immunodeficiency virus and parameters associated with the efficiency of this technique. Arch Dermatol. 2005;141(10):1220-1224.

12. Caye N, Le Fourn B, Pannier M. [Surgical treatment of facial lipoatrophy]. Ann Chir Plast Esthet. 2003;48(1):2-12.

13. Cervelli D, Gasparini G, Moro A, et al. Lipofilling as refinement procedure in maxillo-mandibular malformations. Acta Otorhinolaryngol Ital. 2016;36(5):368-372.

14. Chang Q, Li J, Dong Z, Liu L, Lu F. Quantitative volumetric analysis of progressive hemifacial atrophy corrected using stromal vascular fractionsupplemented autologous fat grafts. Dermatol Surg. 2013;39(10):1465-1473.

15. Denadai R, Raposo-Amaral CA, Buzzo CL, RaposoAmaral CE. Isolated Autologous Free Fat Grafting for Management of Facial Contour Asymmetry in a Subset of Growing Patients With Craniofacial Microsomia. Ann Plast Surg. 2016;76(3):288-294.

16. Denadai R, Raposo-Amaral CA, Pinho AS, Lameiro TM, Buzzo CL, Raposo-Amaral CE. Predictors of Autologous Free Fat Graft Retention in the Management of Craniofacial Contour Deformities. Plast Reconstr Surg. 2017;140(1):50e-61e.

17. Dollfus C, Blanche S, Trocme N, Funck-Brentano I, Bonnet F, Levan P. Correction of facial lipoatrophy using autologous fat transplants in HIV-infected adolescents. HIV Med. 2009;10(5):263-268.

18. Ducic Y, Pontius AT, Smith JE. Lipotransfer as an adjunct in head and neck reconstruction. Laryngoscope. 2003;113(9):1600-1604.

19. Fontdevila J, Guisantes E, Martínez E, Prades E, Berenguer J. Double-blind clinical trial to compare autologous fat grafts versus autologous fat grafts with PDGF: no effect of PDGF. Plastic and reconstructive surgery. 2014;134(2):219e-230e.

20. Fontdevila J, Serra-Renom JM, Raigosa $M$, et al. Assessing the long-term viability of facial fat grafts: an objective measure using computed tomography. Aesthetic surgery journal. 2008;28(4):380-386.

21. Gentile P, De Angelis B, Pasin M, et al. Adiposederived stromal vascular fraction cells and plateletrich plasma: basic and clinical evaluation for cellbased therapies in patients with scars on the face. $J$ Craniofac Surg. 2014;25(1):267-272.

22. Gu Z, Li Y, Li H. Use of Condensed Nanofat Combined With Fat Grafts to Treat Atrophic Scars. JAMA Facial Plast Surg. 2017.

23. Guaraldi G, De Fazio D, Orlando G, et al. Facial lipohypertrophy in HIV-infected subjects who underwent autologous fat tissue transplantation. Clin Infect Dis. 2005;40(2):e13-15.

24. Gutiérrez Santamaría J, Masiá Gridilla J, Pamias Romero J, Giralt López-de-Sagredo J, Bescós Atín MS. Fat grafting is a feasible technique for the sequelae of head and neck cancer treatment. Journal of Cranio-Maxillofacial Surgery. 2017;45(1):93-98.

25. Jiang $T$, Xie $Y$, Zhu M, et al. The second fat graft has significantly better outcome than the first fat graft for Romberg syndrome: A study of threedimensional volumetric analysis. J Plast Reconstr Aesthet Surg. 2016;69(12):1621-1626.

26. Jianhui $Z$, Chenggang $Y$, Binglun $L$, et al. Autologous fat graft and bone marrow-derived mesenchymal stem cells assisted fat graft for treatment of ParryRomberg syndrome. Ann Plast Surg. 2014;73 Suppl 1:S99-103.

27. Jones $C M$, Morrow $B T$, Albright $W B$, Long RE, Samson TD, Mackay DR. Structural Fat Grafting to Improve Reconstructive Outcomes in Secondary Cleft Lip Deformity. Cleft Palate Craniofac J. 2017;54(1):70-74.

28. Koh KS, Oh TS, Kim H, Chung IW, Lee KW, Lee HB. Clinical application of human adipose tissue-derived 
mesenchymal stem cells in progressive hemifacial atrophy (Parry-Romberg disease) with microfat grafting techniques using 3-dimensional computed tomography and 3-dimensional camera. Annals of plastic surgery. 2012;69(3):331-337.

29. Laurent F, Capon-Degardin N, Martinot-Duquennoy $\mathrm{V}$, Dhellemmes P, Pellerin P. [Role of lipo-filling in the treatment of sequelae in craniosynostosis surgery]. Ann Chir Plast Esthet. 2006;51(6):512-516.

30. Lei H, Ma GE, Liu Z. Evaluation of Repairing Facial Depression Deformities Secondary to Lupus Erythematosus Panniculitis With Autologous Fat Grafting. J Craniofac Surg. 2016;27(7):1765-1769.

31. Li GKH, Chung JHP, Liu LHL, Chow VLY, Lau GISK, Chan RCL. Fat grafting: A safe and effective treatment of craniofacial depression. Surgical Practice. 2015;19(2):75-81.

32. Lim AA, Fan K, Allam KA, et al. Autologous fat transplantation in the craniofacial patient: the UCLA experience. J Craniofac Surg. 2012;23(4):10611066.

33. Martins de Carvalho F, Casal D, Bexiga J, et al. HIVAssociated Facial Lipodystrophy: Experience of a Tertiary Referral Center With Fat and Dermis-Fat Compound Graft Transfer. Eplasty. 2016;16:e31.

34. Mojallal A, Shipkov C, Braye F, Breton P, Foyatier JL. Influence of the recipient site on the outcomes of fat grafting in facial reconstructive surgery. Plast Reconstr Surg. 2009;124(2):471-483.

35. Mori A, Lo Russo G, Agostini T, Pattarino J, Vichi F, Dini $M$. Treatment of human immunodeficiency virus-associated facial lipoatrophy with lipofilling and submalar silicone implants. J Plast Reconstr Aesthet Surg. 2006;59(11):1209-1216.

36. Nelson L, Stewart KJ. Experience in the treatment of HIV-associated lipodystrophy. J Plast Reconstr Aesthet Surg. 2008;61(4):366-371.

37. Nelson L, Stewart KJ. Psychological morbidity and facial volume in HIV lipodystrophy: Quantification of treatment outcome. Journal of Plastic, Reconstructive and Aesthetic Surgery. 2012;65(4):439-447.

38. Orlando G, Guaraldi G, De Fazio D, et al. Long-term psychometric outcomes of facial lipoatrophy therapy: forty-eight-week observational, nonrandomized study. AIDS Patient Care STDS. 2007;21(11):833-842.

39. Pallua N, Baroncini A, Alharbi Z, Stromps JP. Improvement of facial scar appearance and microcirculation by autologous lipofilling. J Plast Reconstr Aesthet Surg. 2014;67(8):1033-1037.

40. Phulpin B, Gangloff P, Tran N, Bravetti P, Merlin JL, Dolivet $G$. Rehabilitation of irradiated head and neck tissues by autologous fat transplantation. Plast Reconstr Surg. 2009;123(4):1187-1197.

41. Pinski KS, Roenigk HH, Jr. Autologous fat transplantation. Long-term follow-up. J Dermatol Surg Oncol. 1992;18(3):179-184.

42. Piombino P, Marenzi G, Dell'Aversana Orabona G, Califano L, Sammartino G. Autologous fat grafting in facial volumetric restoration. J Craniofac Surg. 2015;26(3):756-759.

43. Qiao J, Gui L, Fu X, et al. A Novel Method of Mild to Moderate Parry-Romberg Syndrome Reconstruction: Computer-Assisted Surgery With Mandibular Outer Cortex and Fat Grafting. J Craniofac Surg. 2017;28(2):359-365.

44. Rauso R, Curinga G, Santillo V, Corvo G, Tartaro G. Comparison between lipofilling and $a$ nonabsorbable filler for facial wasting rehabilitation in HIV-positive patients. J Craniofac Surg. 2011;22(5):1684-1688.

45. Rauso R, Sangiovanni V, Cobellis G, Tartaro G. Lipofilling's role in facial wasting rehabilitation of $\mathrm{HIV}_{+}$patients. European Surgical Research. 2010;45(3-4):285-286.

46. Sardesai MG, Moore CC. Quantitative and qualitative dermal change with microfat grafting of facial scars. Otolaryngol Head Neck Surg. 2007;137(6):868-872.

47. Schmitz S, Weis C, Morley S, Demey A, Dabernig J. Treatment of facial lipodystrophy syndromes. Lipofilling versus free flap surgery. European Journal of Plastic Surgery. 2008;31(6):305-310.

48. Scotto di Santolo M, Sagnelli M, Tortora G, et al. The utility of the high-resolution ultrasound technique in the evaluation of autologous adipose tissue lipofilling, used for the correction of postsurgical, post-traumatic and post-burn scars. La Radiologia medica. 2016;121(6):521-527.

49. Serra-Renom JM, Fontdevila J. Treatment of facial fat atrophy related to treatment with protease inhibitors by autologous fat injection in patients with human immunodeficiency virus infection. Plast Reconstr Surg. 2004;114(2):551-555; discussion 556557.

50. Slack GC, Tabit CJ, Allam KA, Kawamoto HK, Bradley JP. Parry-Romberg reconstruction: beneficial results despite poorer fat take. Ann Plast Surg. 2014;73(3):307-310.

51. Sterodimas A, Faria J, Nicaretta B, Boriani F. Autologous fat transplantation versus adiposederived stem cell-enriched lipografts: a study. Aesthetic surgery journal. 2011;31(6):682-693.

52. Tanikawa DY, Aguena M, Bueno DF, Passos-Bueno MR, Alonso N. Fat grafts supplemented with adipose-derived stromal cells in the rehabilitation of patients with craniofacial microsomia. Plast Reconstr Surg. 2013;132(1):141-152.

53. Tanna N, Wan DC, Kawamoto HK, Bradley JP. Craniofacial microsomia soft-tissue reconstruction comparison: inframammary extended circumflex scapular flap versus serial fat grafting. Plast Reconstr Surg. 2011;127(2):802-811.

54. Uzzan C, Boccara D, Lachere A, Mimoun M, Chaouat M. [Treatment of facial lipoatrophy by lipofilling in HIV infected patients: retrospective study on 317 patients on 9 years]. Ann Chir Plast Esthet. 2012;57(3):210-216.

55. Viard R, Bouguila J, Voulliaume D, Comparin JP, Dionyssopoulos A, Foyatier JL. [Fat grafting in facial 
burns sequelae]. Ann Chir Plast Esthet. 2012;57(3):217-229.

56. Vitagliano T, Curto LS, Greto Ciriaco A, Gareri P, Ribuffo D, Greco M. Two-Thirds Lip Defects: A New Combined Reconstructive Technique for Patients With Epithelial Cancer. J Craniofac Surg. 2016;27(8):1995-2000.

57. Xie Y, Li Q, Zheng D, Lei H, Pu LL. Correction of hemifacial atrophy with autologous fat transplantation. Ann Plast Surg. 2007;59(6):645653.

58. Yang X, Wu R, Bi H, et al. Autologous Fat Grafting With Combined Three-Dimensional and MirrorImage Analyses for Progressive Hemifacial Atrophy. Ann Plast Surg. 2016;77(3):308-313.

59. Yoshimura K, Sato K, Aoi N, et al. Cell-assisted lipotransfer for facial lipoatrophy: efficacy of clinical use of adipose-derived stem cells. Dermatol Surg. 2008;34(9):1178-1185.

6o. Coleman SR. Structural fat grafting. Aesthetic surgery journal / the American Society for Aesthetic Plastic surgery. 1998;18(5):386, 388. 


\subsection{Online supplement}

\subsubsection{E-figures}

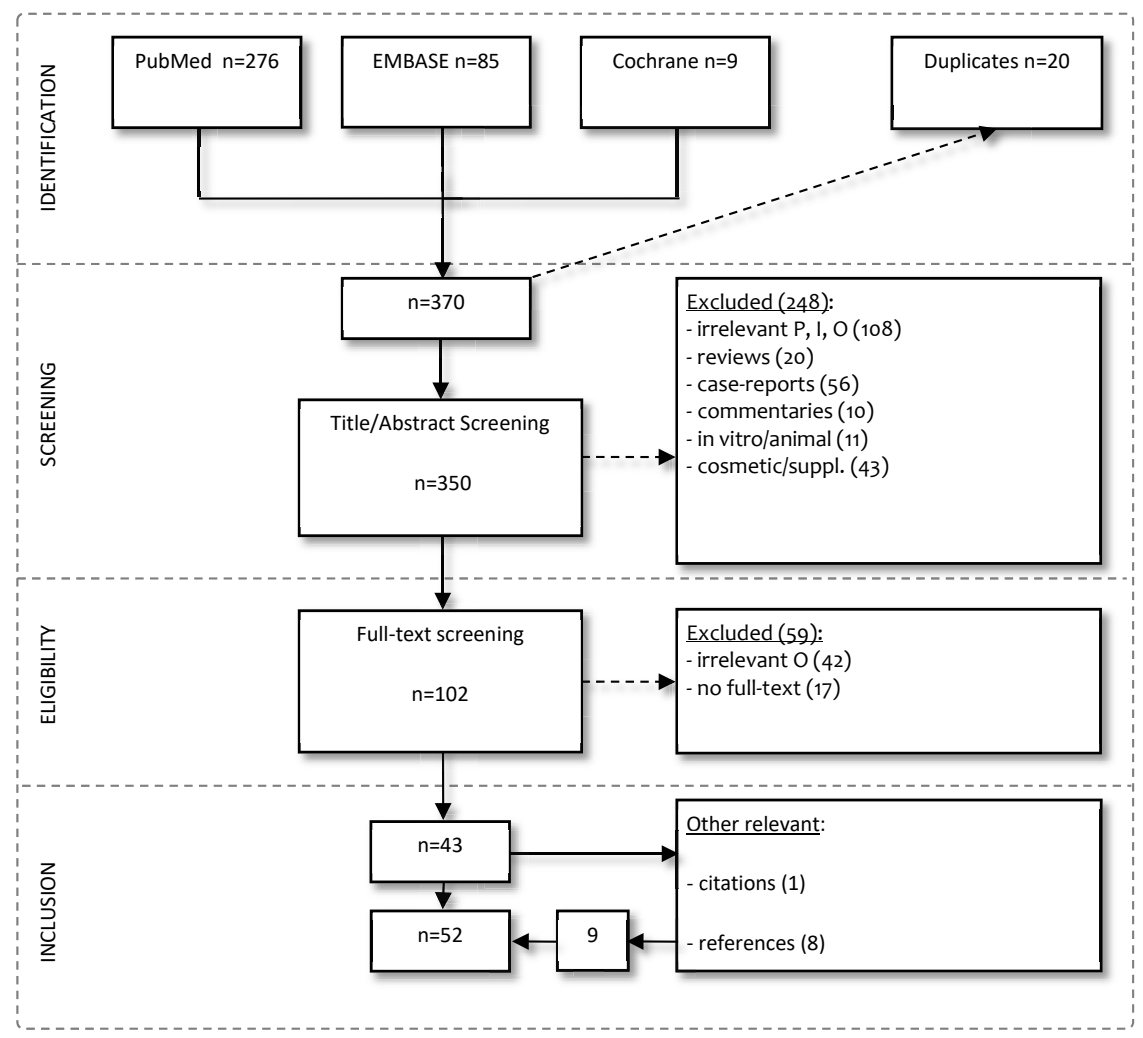

eFigure 1: Flowchart of the search strategy. Legend: $n=$ number of studies; $P=$ Population, $I=$ Intervention, $O=$ Outcome, suppl.=supplements (e.g. $P R P$, stromal-vascular fraction enrichment) 


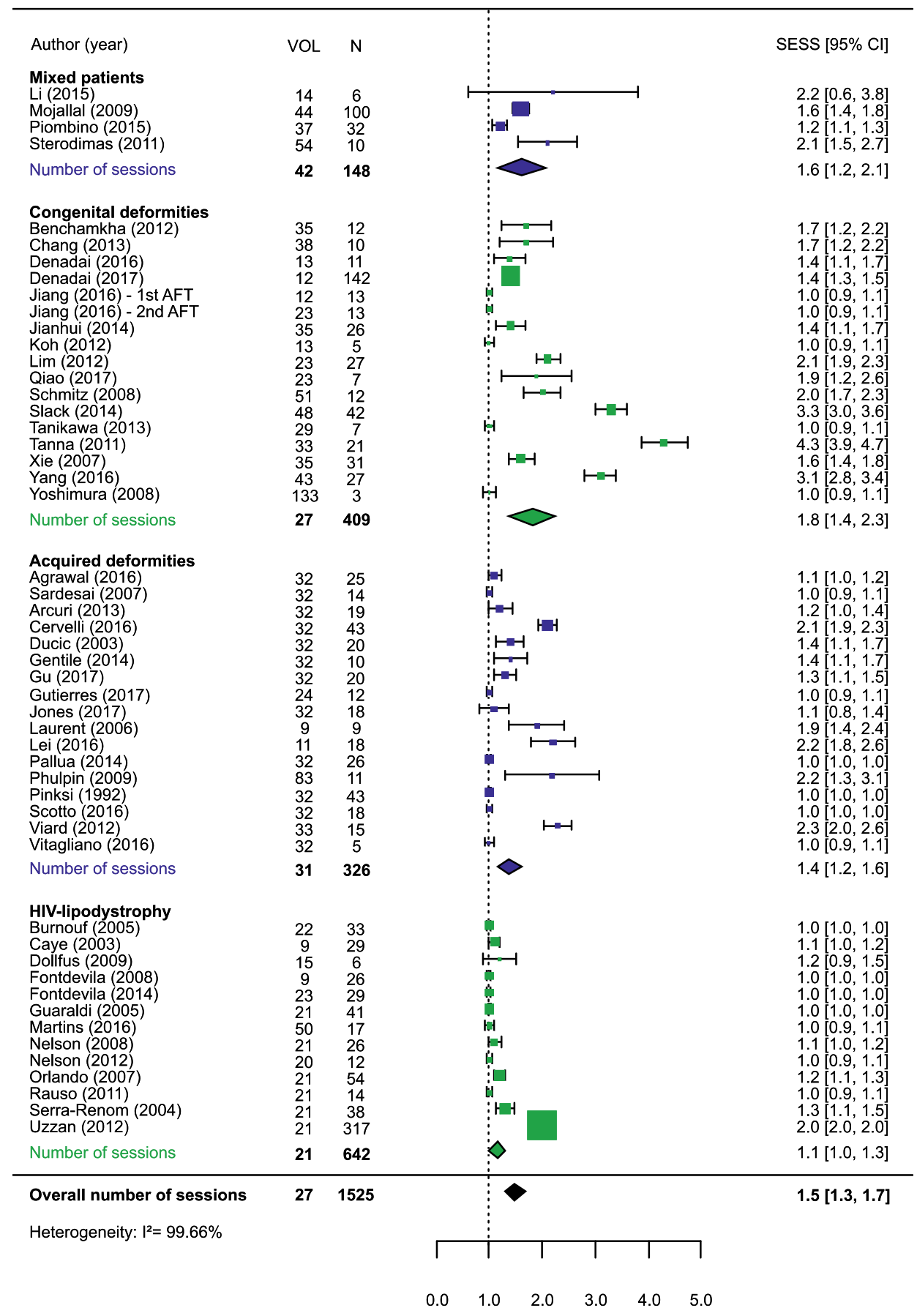

Mean number of AFT sessions

eFigure 2: Meta-analysis of the number of AFT sessions needed to achieve the desired result. Legend: VOL=mean injected volume. $N=$ total number of patients. 


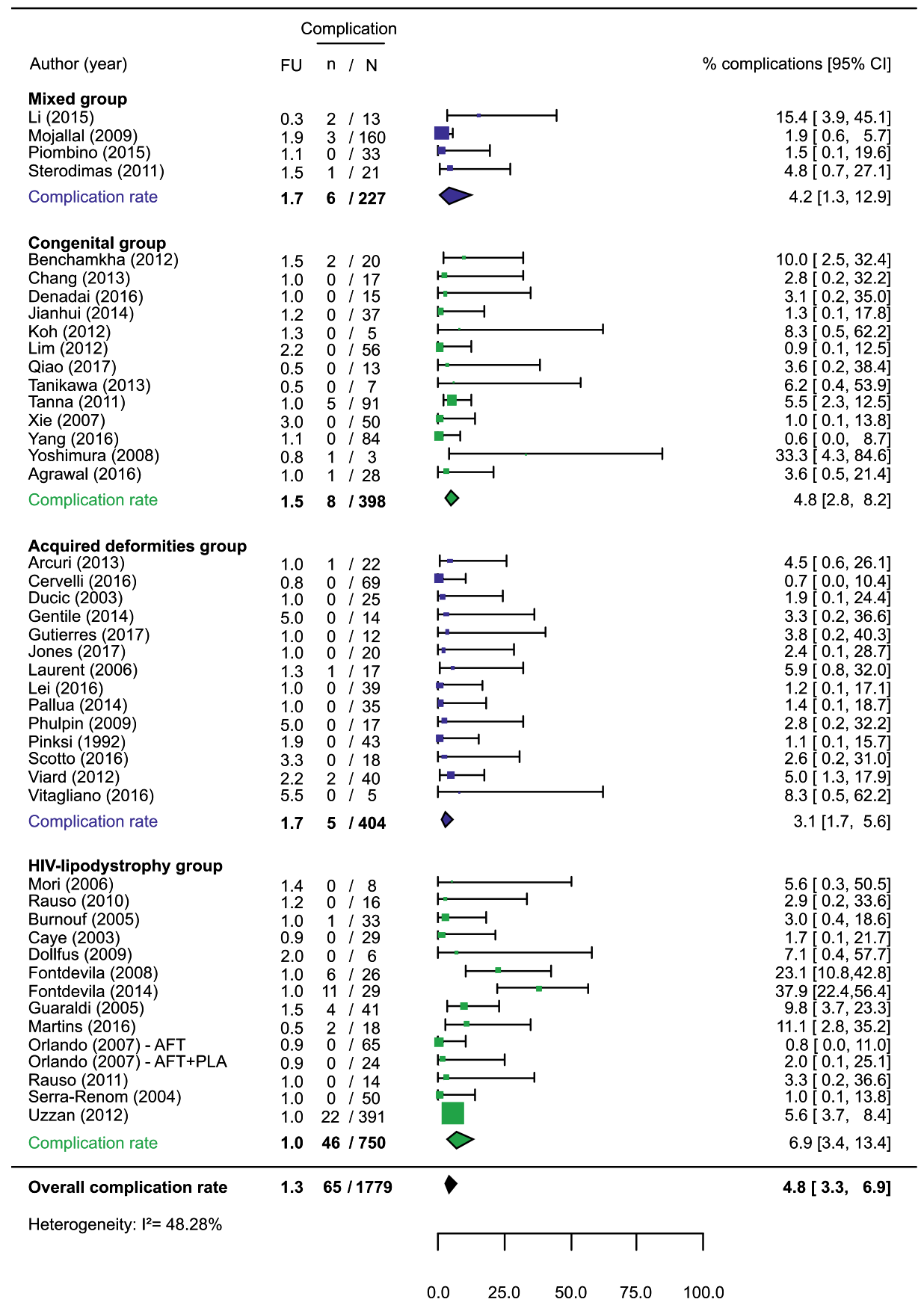

Percentage of AFT-related complications (per procedure)

eFigure 3: Meta-analysis of the rate of AFT-related complications per procedure. Legend: FU=followup, VOL=mean injected volume, $n=$ total number of complications, $N=$ total number of procedures. 


\subsubsection{E-tables}

eTable 1: PICO design

\begin{tabular}{ll}
\hline PICO & Description \\
\hline Population & Patients with craniofacial soft-tissue deformities \\
Intervention & Autologous Fat Transfer (without the use of supplements such as PRP, SVF) \\
Controls & Conventional reconstructive techniques, fillers or no treatment \\
Outcomes & $\begin{array}{l}\text { Crucial outcome measures: } \\
\text { - Patient satisfaction } \\
\end{array}$ \\
& - Surgeon satisfaction/evaluation \\
& Important outcome measures: \\
& - Number of AFT sessions \\
& - Volume retention (degree of resorption)
\end{tabular}

eTable 2: Search syntax

\begin{tabular}{|c|c|}
\hline Database & Search syntax \\
\hline \multirow[t]{16}{*}{ PubMed } & (craniofacial[tiab] $\quad$ OR cranio-facial[tiab] $\quad$ OR cranio[tiab] $\quad$ OR cranial[tiab] OR facial[tiab] OR \\
\hline & hemifacial[tiab] OR face[tiab]) AND (atroph*[tiab] OR lipoatroph*[tiab] OR hypoplas*[tiab] OR \\
\hline & aplas*[tiab] OR defect[tiab] OR deformit*[tiab] OR deficienc*[tiab] OR malformation[tiab] OR \\
\hline & disorder[tiab] OR congenital[tiab] OR hereditary[tiab] OR syndrome[tiab] OR Parry[tiab] OR \\
\hline & Romberg[tiab] OR Treacher[tiab] OR microsomia[tiab] OR atroph*[tiab] OR lipoatroph*[tiab] OR \\
\hline & lipodystrophy*[tiab] OR deficienc*[tiab] OR HIV[tiab] OR immuno-defficiency[tiab] OR AIDS[tiab]) AND \\
\hline & (("fat graft"[tiab] OR "fat grafts"[tiab] OR "fat grafting"[tiab]) OR "fat injection"[tiab] OR \\
\hline & lipoaspirate*[tiab] OR "fat transfer"[tiab] OR lipofilling[tiab] OR liposculptur*[tiab] OR \\
\hline & lipostructur*[tiab] OR lipo-injection[tiab] OR lipoinjection[tiab] OR lipotransfer[tiab] OR lipo- \\
\hline & transfer[tiab] OR "fat transplant"[tiab] $\quad$ OR lipomodelling[tiab] $\quad$ OR lipomodeling[tiab] $O R$ \\
\hline & lipografting[tiab] OR lipo-grafting[tiab] OR microlipoinjection[tiab] OR "adipose tissue grafting"[tiab] \\
\hline & OR "adipose tissue transfer"[tiab] OR "adipose tissue transplant"[tiab] OR lipotransplant[tiab] OR lipo- \\
\hline & transplant[tiab] OR microlipofilling[tiab] OR micro-lipofilling[tiab] OR "grafting of fatty tissue"[tiab] OR \\
\hline & "transfer of fatty tissue"[tiab] OR "injection of fatty tissue"[tiab] OR "fatty tissue grafting"[tiab] OR \\
\hline & "fatty tissue transfer"[tiab] OR "fatty tissue injection"[tiab] OR "autologous fat transplant"[tiab] OR \\
\hline & "autologous fat transplants"[tiab]) NOT ("dermal"[tiab] OR derma-fat[tiab] OR derma[tiab]) \\
\hline Embase & (craniofacial:ti:ab OR cranio-facial:ti:ab ... OR derma:ti:ab) AND [embase]/lim NOT [medline]/lim \\
\hline Cochrane & (craniofacial OR cranio-facial ... OR derma) in Abstract, Title and Keywords \\
\hline
\end{tabular}




\begin{tabular}{|c|c|}
\hline Database & Search syntax \\
\hline Study design characteristics & Assessment of: \\
\hline - Author (year) & overlap studies \\
\hline - Study period (start date - end date) & overlap studies \\
\hline - Type of trial (RCT, cohort, case-series, case-report) & study design \\
\hline - Design (prospective, retrospective) & study design \\
\hline \multicolumn{2}{|l|}{ Evaluation of the risk of bias } \\
\hline - Randomization (if non-RCT, use of consecutive patients)? & Selection bias \\
\hline - Blinding of participants and personnel (if applicable)? & Performance bias \\
\hline - Blinding of outcome assessment (if applicable)? & Detection bias \\
\hline - Incomplete outcome data (loss to follow up >15\%?) & Attrition bias \\
\hline - Selective reporting of outcomes? & Reporting bias \\
\hline - Suspicion of funding by industry, conflict of interests? & Publication bias \\
\hline \multicolumn{2}{|l|}{ Study population characteristics } \\
\hline - Number of treated patients, total number of interventions & Important outcome \\
\hline - Age, BMI & Demographics \\
\hline - Diagnosis, indication, previous procedures & Demographics \\
\hline - Surgical technique, type of anaesthesia, donor sites & Important outcome \\
\hline - Number of AFT sessions, & Important outcome \\
\hline - Mean injected volume per session, per location & Important outcome \\
\hline \multicolumn{2}{|l|}{ Time factor } \\
\hline - Time between AFT sessions & Important outcome \\
\hline - Length of follow-up & Important outcome \\
\hline \multicolumn{2}{|l|}{ Volume assessment } \\
\hline - Type of imaging used, volume measurements & Important outcome \\
\hline \multicolumn{2}{|l|}{ Patient and surgeon satisfaction } \\
\hline - Type of measurement tool/scale/score used, timing evaluation & Crucial outcome \\
\hline \multicolumn{2}{|l|}{ Quality of life } \\
\hline - Type of scoring system used, time evaluation & Crucial outcome \\
\hline \multicolumn{2}{|l|}{ Number of sessions } \\
\hline - Number of AFT procedures per patient needed for successful reconstruction & Important outcome \\
\hline \multicolumn{2}{|l|}{ Complications } \\
\hline - Type and total number of AFT-related complications & Crucial outcome \\
\hline
\end{tabular}




\begin{tabular}{|c|c|c|c|c|c|c|c|c|c|c|c|c|c|c|c|c|c|}
\hline & Study & 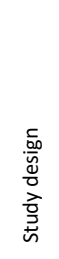 & 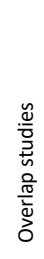 & 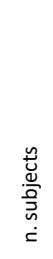 & 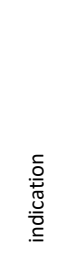 & 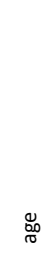 & 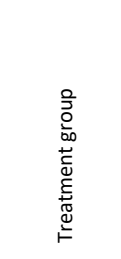 & 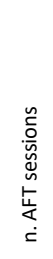 & 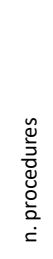 & 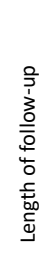 & 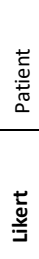 & 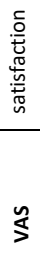 & 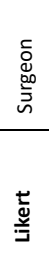 & 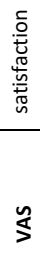 & 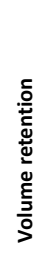 & 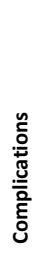 & 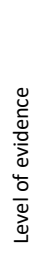 \\
\hline 1 & Agrawal (2016) & $\mathrm{CS}$ & no & 25 & acq. & 30 & AFT & 1.1 & 28 & 1.0 & & $\checkmark$ & & $\checkmark$ & $\checkmark$ & $\checkmark$ & 4 \\
\hline 2 & Arcuri (2013) & $\mathrm{CS}$ & no & 19 & acq. & 30 & AFT & 1.2 & 22 & 1.0 & & & $\checkmark$ & & & $\checkmark$ & 4 \\
\hline 3 & Benchamkha (2012) & $\mathrm{CS}$ & no & 12 & PRS & 32 & AFT & 1.7 & 20 & 1.5 & $\checkmark$ & & & & & $\checkmark$ & 4 \\
\hline 4 & Burnouf (2005) & CS & no & 33 & HIV & 45 & AFT & 1.0 & 33 & 1.0 & $\checkmark$ & & $\checkmark$ & & & $\checkmark$ & 4 \\
\hline 5 & Caye (2003) & CS & no & 29 & HIV & 43 & AFT & 1.1 & 29 & 0.9 & & & $\checkmark$ & & & $\checkmark$ & 4 \\
\hline 6 & Cervelli (2016) & CS & no & 43 & acq. & 30 & AFT & 2.1 & 69 & 0.8 & & & & & & $\checkmark$ & 4 \\
\hline \multirow[t]{2}{*}{7} & Chang (2013) & $\mathrm{CH}$ & no & 10 & PRS & 28 & $A F T+S V F$ & 1.7 & 17 & 1.0 & & & & & $\checkmark$ & $\checkmark$ & 2 \\
\hline & & & & 10 & PRS & 28 & AFT & 1.7 & 17 & 1.0 & & & & & $\checkmark$ & $\checkmark$ & \\
\hline 8 & Denadai (2016) & CS & no & 11 & $\mathrm{CM}$ & 6 & AFT & 1.4 & 15 & 1.0 & & $\checkmark$ & & $\checkmark$ & & $\checkmark$ & 4 \\
\hline 9 & Denadai (2017) & CS & no & 142 & $\mathrm{CM}$ & 22 & AFT & 1.4 & 205 & 0.0 & & & & & $\checkmark$ & $\checkmark$ & 4 \\
\hline 10 & Dollfus (2009) & CS & no & 6 & HIV & 17 & AFT & 1.2 & 6 & 2.0 & $\checkmark$ & & & & & $\checkmark$ & 4 \\
\hline 11 & Ducic (2003) & CS & no & 20 & acq. & & AFT & 1.4 & 25 & 1.0 & & & $\checkmark$ & & & $\checkmark$ & 4 \\
\hline 12 & Fontdevila (2008) & CS & no & 26 & HIV & 45 & AFT & 1.0 & 26 & 1.0 & & & & & $\checkmark$ & $\checkmark$ & 4 \\
\hline \multirow[t]{2}{*}{13} & Fontdevila (2014) & $\mathrm{RCT}$ & no & 29 & HIV & 46 & AFT & 1.0 & 29 & 1.0 & & & $\checkmark$ & & $\checkmark$ & $\checkmark$ & 1 \\
\hline & & & & 20 & HIV & 47 & $A F T+P R P$ & 1.0 & 20 & 1.0 & & & $\checkmark$ & & $\checkmark$ & $\checkmark$ & \\
\hline \multirow[t]{3}{*}{14} & Gentile (2014) & $\mathrm{CH}$ & no & 10 & acq. & 45 & $\mathrm{AFT}+\mathrm{SVF}$ & 1.4 & 14 & 5.0 & $\checkmark$ & & & & $\checkmark$ & $\checkmark$ & 2 \\
\hline & & & & 10 & acq. & 45 & AFT+PRP & 1.4 & 14 & 5.0 & $\checkmark$ & & & & $\checkmark$ & $\checkmark$ & \\
\hline & & & & 10 & acq. & 45 & AFT & 1.4 & 14 & 5.0 & $\checkmark$ & & & & $\checkmark$ & $\checkmark$ & \\
\hline 15 & Gutierres (2017) & CS & no & 12 & acq. & 60 & AFT & 1.0 & 12 & 1.0 & & & $\checkmark$ & $\checkmark$ & & $\checkmark$ & 4 \\
\hline 16 & Gu (2017) & $\mathrm{CS}$ & no & 20 & acq. & 38 & AFT & 1.3 & 26 & 0.0 & & $\checkmark$ & & $\checkmark$ & & & 4 \\
\hline 17 & Guaraldi (2005) & CS & no & 41 & HIV & & AFT & 1.0 & 41 & 1.5 & & & & & $\checkmark$ & & 4 \\
\hline 18 & Jiang (2016) & $\mathrm{CS}$ & no & 26 & PRS & 27 & AFT & 2.0 & 52 & - & & $\checkmark$ & & $\checkmark$ & $\checkmark$ & & 4 \\
\hline \multirow[t]{2}{*}{19} & Jianhui (2014) & $\mathrm{CH}$ & no & 26 & PRS & 24 & AFT & 1.4 & 37 & 1.2 & & & $\checkmark$ & & & $\checkmark$ & 3 \\
\hline & & & & 10 & PRS & 25 & $\mathrm{AFT}+\mathrm{BMSC}$ & 1.0 & 10 & 1.2 & & & $\checkmark$ & & & $\checkmark$ & \\
\hline 20 & Jones (2017) & CS & no & 18 & acq. & 16 & AFT & 1.1 & 20 & 1.0 & & $\checkmark$ & & $\checkmark$ & & $\checkmark$ & 4 \\
\hline \multirow[t]{2}{*}{21} & Koh (2012) & $\mathrm{RCT}$ & no & 5 & PRS & 28 & AFT & 1.0 & 5 & 1.5 & & & & & $\checkmark$ & & 1 \\
\hline & & & & 5 & PRS & 28 & $A F T+S V F$ & 1.0 & 5 & 1.5 & & & & & $\checkmark$ & & \\
\hline 22 & Laurent (2006) & CS & no & 9 & acq. & 16 & AFT & 1.9 & 17 & 1.3 & $\checkmark$ & & $\checkmark$ & & & $\checkmark$ & 4 \\
\hline 23 & Lei (2016) & $\mathrm{CS}$ & no & 18 & acq. & 37 & AFT & 2.2 & 39 & 1.5 & $\checkmark$ & & $\checkmark$ & & & $\checkmark$ & 4 \\
\hline 24 & Li (2015) & $\mathrm{CS}$ & no & 6 & $\operatorname{mix}$ & 47 & AFT & 2.2 & 13 & 0.3 & & $\checkmark$ & & $\checkmark$ & & $\checkmark$ & 4 \\
\hline 25 & $\operatorname{Lim}(2012)$ & $\mathrm{CS}$ & no & 27 & $\mathrm{CM}$ & 17 & AFT & 2.1 & 56 & 2.2 & & & & & & $\checkmark$ & 4 \\
\hline
\end{tabular}




\begin{tabular}{|c|c|c|c|c|c|c|c|c|c|c|c|c|c|c|c|c|c|}
\hline & Study & 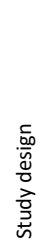 & 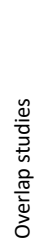 & 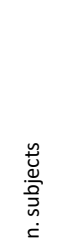 & 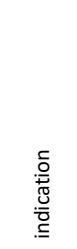 & 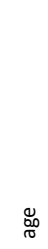 & 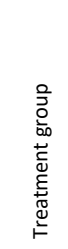 & 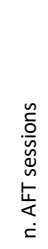 & 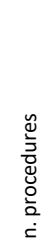 & 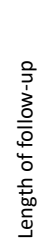 & 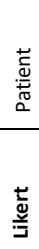 & 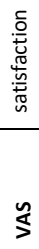 & 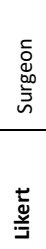 & 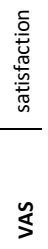 & 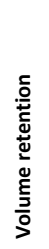 & 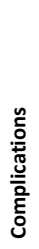 & 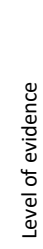 \\
\hline \multirow[t]{2}{*}{26} & Martins (2016) & $\mathrm{CH}$ & no & 18 & HIV & 51 & AFT & 1.0 & 18 & 0.5 & & $\checkmark$ & & & & $\checkmark$ & 3 \\
\hline & & & & 6 & HIV & 49 & DFG & 1.0 & 6 & 0.0 & & $\checkmark$ & & & & & \\
\hline 27 & Mojallal (2009) & CS & no & 100 & $\operatorname{mix}$ & 42 & AFT & 1.6 & 160 & 1.9 & $\checkmark$ & $\checkmark$ & & $\checkmark$ & & $\checkmark$ & 4 \\
\hline \multirow[t]{2}{*}{28} & Mori (2006) & $\mathrm{CH}$ & no & 8 & HIV & - & AFT & - & 8 & - & $\checkmark$ & & & & & $\checkmark$ & 3 \\
\hline & & & & 4 & HIV & - & Impl. & - & 4 & - & $\checkmark$ & & & & & & \\
\hline \multirow[t]{3}{*}{29} & Nelson (2008) & $\mathrm{CH}$ & no & 26 & HIV & - & AFT & 1.1 & 26 & - & & $\checkmark$ & & & $\checkmark$ & & 2 \\
\hline & & & & 10 & HIV & - & PLA & - & 10 & - & & $\checkmark$ & & & & & \\
\hline & & & & 8 & HIV & - & Bio-A & - & 8 & - & & $\checkmark$ & & & & & \\
\hline \multirow[t]{3}{*}{30} & Nelson (2012) & $\mathrm{CH}$ & no & 12 & HIV & 43 & AFT & 1.0 & 12 & - & & $\checkmark$ & & & $\checkmark$ & & 2 \\
\hline & & & & 16 & HIV & 45 & Bio-A & - & 16 & - & & $\checkmark$ & & & $\checkmark$ & & \\
\hline & & & & 20 & HIV & 46 & PLA & - & 20 & - & & $\checkmark$ & & & & & \\
\hline \multirow[t]{4}{*}{31} & Orlando (2007) & $\mathrm{CH}$ & no & 54 & HIV & 45 & AFT & 1.2 & 65 & 0.9 & & $\checkmark$ & & & & $\checkmark$ & 2 \\
\hline & & & & 24 & HIV & 47 & AFT & 1.0 & 24 & 0.9 & & $\checkmark$ & & & & $\checkmark$ & \\
\hline & & & & 91 & HIV & 46 & PLA & - & 91 & - & & $\checkmark$ & & & & & \\
\hline & & & & 130 & HIV & 46 & PAcr & - & 130 & - & & $\checkmark$ & & & & & \\
\hline 32 & Pallua (2014) & CS & no & 26 & acq. & 46 & AFT & 1.0 & 35 & 1.9 & & $\checkmark$ & & $\checkmark$ & & $\checkmark$ & 4 \\
\hline 33 & Phulpin (2009) & CS & no & 11 & acq. & 48 & AFT & 2.2 & 17 & 3.3 & & & & $\checkmark$ & & $\checkmark$ & 4 \\
\hline 34 & Pinksi (1992) & CS & no & 43 & acq. & 35 & AFT & 1.0 & 43 & 2.2 & & & & & & $\checkmark$ & 4 \\
\hline 35 & Piombino (2015) & CS & no & 32 & $\operatorname{mix}$ & 24 & AFT & 1.2 & 33 & 1.1 & & $\checkmark$ & $\checkmark$ & & & $\checkmark$ & 4 \\
\hline 36 & Qiao (2017) & CS & no & 7 & PRS & 22 & AFT & 1.9 & 11 & 0.5 & $\checkmark$ & $\checkmark$ & $\checkmark$ & & $\checkmark$ & $\checkmark$ & 4 \\
\hline 37 & Rauso (2010) & $\mathrm{CS}$ & 38 & 16 & HIV & - & AFT & - & 16 & 1.2 & & & & & & $\checkmark$ & 4 \\
\hline \multirow[t]{2}{*}{38} & Rauso (2011) & $\mathrm{CH}$ & 37 & 14 & HIV & 44 & AFT & 1.0 & 14 & 1.0 & & & & & & $\checkmark$ & 2 \\
\hline & & & & 9 & HIV & 45 & Bio-A & - & 9 & 1.0 & & & & & & $\checkmark$ & \\
\hline 39 & Sardesai (2007) & CS & no & 14 & acq. & 48 & AFT & 1.0 & - & - & & $\checkmark$ & & $\checkmark$ & & & 4 \\
\hline \multirow[t]{2}{*}{40} & Schmitz (2008) & $\mathrm{CH}$ & no & 12 & PRS & - & AFT & 2.0 & 24 & - & $\checkmark$ & & & & & & 3 \\
\hline & & & & 7 & PRS & - & flap & - & 7 & - & $\checkmark$ & & & & & & \\
\hline 41 & Scotto (2016) & CS & no & 18 & acq. & 38 & AFT & 1.0 & 18 & 1.0 & & & & & & & 4 \\
\hline 42 & Serra-Renom (2004) & CS & no & 38 & HIV & 42 & AFT & 1.3 & 50 & 1.0 & & $\checkmark$ & & $\checkmark$ & & $\checkmark$ & 4 \\
\hline \multirow[t]{2}{*}{43} & Slack (2014) & $\mathrm{CH}$ & no & 42 & PRS & - & AFT & 3.3 & 139 & - & & & & & $\checkmark$ & & 3 \\
\hline & & & & 35 & cosm. & - & AFT & 1.7 & 60 & - & & & & & $\checkmark$ & & \\
\hline
\end{tabular}




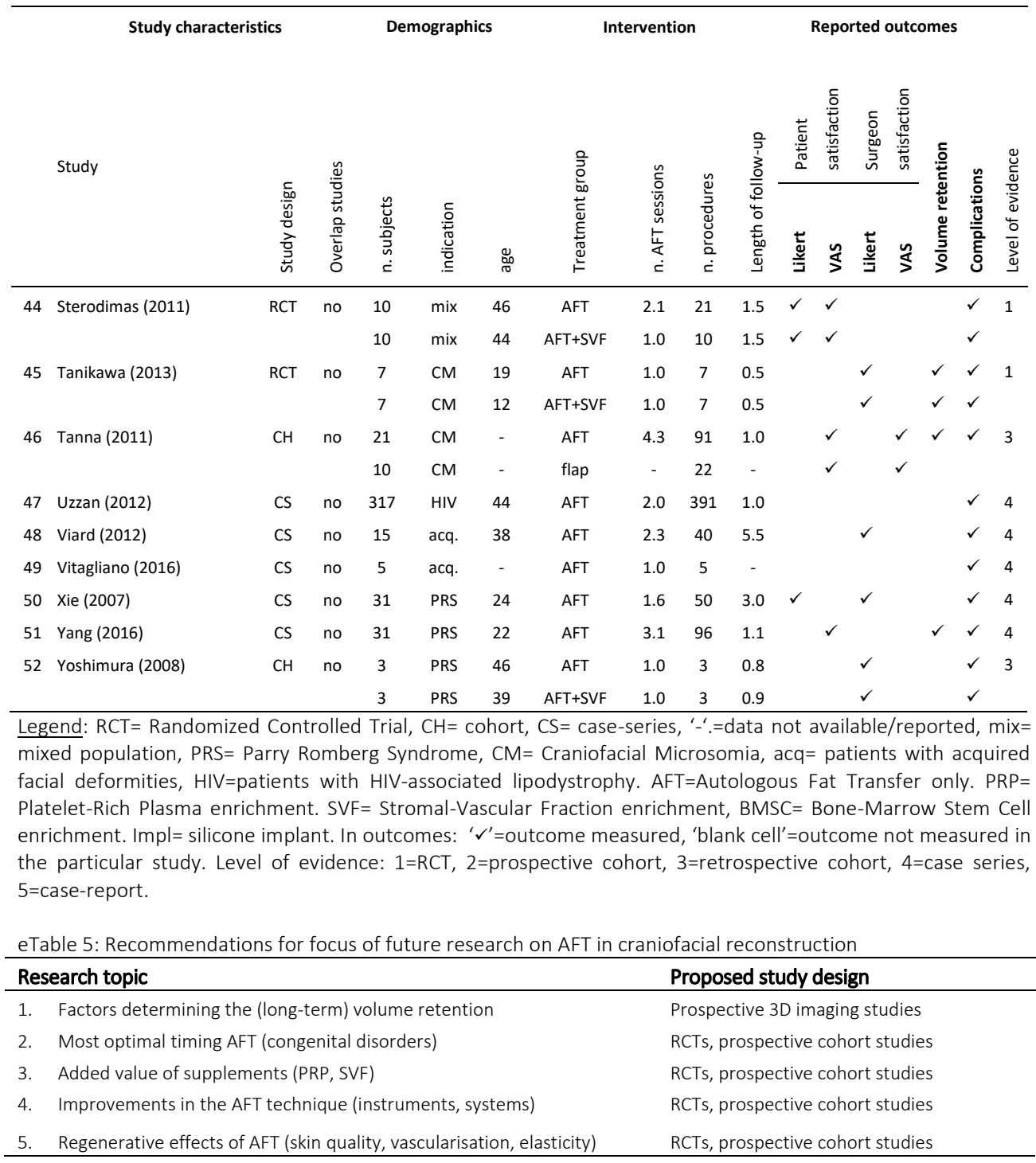




\begin{tabular}{llll}
\hline Section/Topic & \# & Checklist item & page \# \\
\hline Title & & & \\
Title & 1 & Identify the report as a systematic review, meta-analysis, or both. & \\
\hline Abstract &
\end{tabular}

\section{Abstract}

Structured summary 2 Provide a structured summary including, as applicable: background; objectives; data sources; study eligibility criteria, participants, and interventions; study appraisal and synthesis methods; results; limitations; conclusions and implications of key findings; systematic review registration number.

\section{Introduction}

Rationale

Objectives

\section{Methods}

Protocol and

registration

Eligibility criteria

Information sources

Search

Study selection

Data collection process

Data items

Risk of bias in individual studies

Summary measures Synthesis of results

Risk of bias across studies

Additional analyses

\section{Results}

Study selection

Study characteristics

Risk of bias within

studies

Results of individual studies

Synthesis of results

Risk of bias across

studies

Additional analysis
3 Describe the rationale for the review in the context of what is already known. 4

4 Provide an explicit statement of questions being addressed with reference to 4 participants, interventions, comparisons, outcomes, and study design (PICOS).

5 Indicate if a review protocol exists, if and where it can be accessed (e.g., Web N.A. address), and, if available, provide registration information including registration number.

6 Specify study characteristics (e.g., PICOS, length of follow-up) and report characteristics (e.g., years considered, language, publication status) used as criteria for eligibility, giving rationale

7 Describe all information sources (e.g., databases with dates of coverage, contact with study authors to identify additional studies) in the search and date last searched.

8 Present full electronic search strategy for at least one database, including any limits used, such that it could be repeated.

9 State the process for selecting studies (i.e., screening, eligibility, included in systematic review, and, if applicable, included in the meta-analysis).

10 Describe method of data extraction from reports (e.g., piloted forms, independently, in duplicate) and any processes for obtaining and confirming data from investigators.

11 List and define all variables for which data were sought (e.g., PICOS, funding 5 sources) and any assumptions and simplifications made.

12 Describe methods used for assessing risk of bias of individual studies (including specification of whether this was done at the study or outcome level), and how this information is to be used in any data synthesis.

13 State the principal summary measures (e.g., risk ratio, difference in means). 5

14 Describe the methods of handling data and combining results of studies, if 5 done, including measures of consistency (e.g., $I^{2}$ ) for each meta-analysis.

15 Specify any assessment of risk of bias that may affect the cumulative evidence 5 (e.g., publication bias, selective reporting within studies).

16 Describe methods of additional analyses (e.g., sensitivity or subgroup analyses, 5 meta-regression), if done, indicating which were pre-specified.

17 Give numbers of studies screened, assessed for eligibility, and included in the 5 review, with reasons for exclusions at each stage, ideally with a flow diagram.

8 For each study, present characteristics for which data were extracted (e.g., 6 study size, PICOS, follow-up period) and provide the citations.

19 Present data on risk of bias of each study and, if available, any outcome level 6 assessment (see item 12).

20 For all outcomes considered (benefits or harms), present, for each study: (a) 8-9 simple summary data for each intervention group (b) effect estimates and confidence intervals, ideally with a forest plot.

21 Present results of each meta-analysis done, including confidence intervals and 8-9 measures of consistency.

22 Present results of any assessment of risk of bias across studies (see Item 15). 9-10

23 Give results of additional analyses, if done (e.g., sensitivity or subgroup 8-9 
analyses, meta-regression [see Item 16]).

\section{Discussion}

Summary of

evidence

Limitations

Conclusions
24 Summarize the main findings including the strength of evidence for each main outcome; consider their relevance to key groups (e.g., healthcare providers, users, and policy makers).

25 Discuss limitations at study and outcome level (e.g., risk of bias), and at 10 review-level (e.g., incomplete retrieval of identified research, reporting bias).

26 Provide a general interpretation of the results in the context of other evidence, and implications for future research.

\section{Funding}

Funding

9
27 Describe sources of funding for the systematic review and other support (e.g., supply of data); role of funders for the systematic review.
From: Moher D, Liberati A, Tetzlaff J, Altman DG, The PRISMA Group (2009). Preferred Reporting Items for Systematic Reviews and Meta-Analyses: The PRISMA Statement. PLoS Med 6(6): e1000097. doi:10.1371/journal.pmed1000097 For more information, visit: www. prisma-statement.org. 


\section{CHAPTER 3}

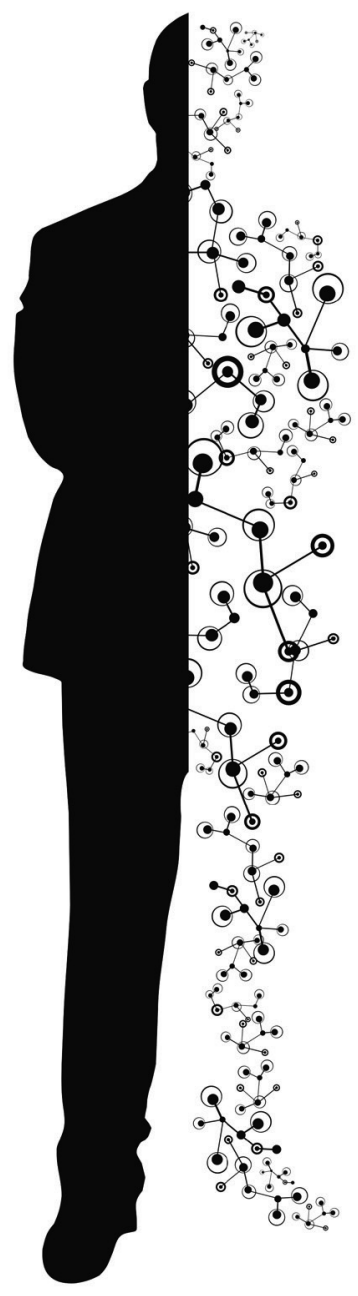




\section{Autologous Fat Transfer as a Treatment for Peripheral Neuropathic Pain without Apparent Cause}

Beugels J, Hommes JE, Balthasar AJR, van der Hulst RRWJ, Piatkowski de Grzymala AA. 


\section{Summary}

Neuropathic pain has a far-reaching effect on the daily lives of patients. Recently, autologous fat transfer (AFT) has demonstrated promising results in patients with painful scars or after neuroma excision. However, there is a subgroup of patients who do not show any apparent cause for the pain. We hypothesized that in these patients, AFT alone in the area around the affected nerve might lead to beneficial results. Patients with clearly demarcated neuropathic pain and who had exhausted all other treatment options were referred by a pain specialist. Fourteen patients who met the inclusion criteria received AFT in the area of the affected nerve. Pain scored on the VAS, patient satisfaction and quality of sleep were recorded before and after surgery. To investigate long-term effects, a second follow-up was planned at least one year later. Patient satisfaction was $93 \%$ after the first follow-up and $86 \%$ after more than one year. The mean VAS score was 7.4 before surgery and significantly decreased to 3.8 after autologous fat grafting $(p<0.0001)$ and $4.3(p=0.0017)$ at long-term follow-up. The quality of sleep improved in $50 \%$ of the patients, whereas the remainder indicated no difference. No complications were registered. The results show that AFT alone, even over a longer period of time and in patients refractory to multiple treatment modalities, could be useful to treat peripheral neuropathic pain without apparent cause. For definitive evidence prospective controlled studies with a larger study population and control/sham groups are warranted.

\section{Introduction}

Neuropathic pain, estimated to occur in $1-8 \%$ of the general population, is a challenge in daily practice with a profound influence on daily life. ${ }^{1}$ Treatment modalities are diverse and include various forms of pharmacotherapy, electrical stimulation and surgical interventions. ${ }^{2-}$ ${ }^{4}$ Pharmacotherapy and other non-surgical treatments lead to a clinically meaningful pain reduction in less than $50 \%$ and $30 \%$ of the cases, respectively. ${ }^{5,6}$ Patients with refractory pain often undergo surgery, which traditionally was mostly performed by resection and relocation of the affected nerve into muscle, vein or bone..$^{7-9}$ In recent years autologous fat transfer (AFT) has been introduced into the arsenal of surgical techniques to treat neuropathic pain caused by neuromas, burns and scars and post mastectomy pain syndrome (PMPS). ${ }^{10-16}$ Acknowledging the beneficial effect of AFT found for these 
indications, our aim was to investigate whether autologous fat transfer can be an effective treatment for peripheral neuropathic pain when there is no clear cause in the form of a neuroma or scar.

\section{Methods}

A retrospective cohort study was conducted among patients with neuropathic pain confined to a specific location without apparent cause who were treated with autologous fat transfer between December 2011 and August 2014.

\section{$\underline{\text { Patient inclusion }}$}

All patients were referred by a pain specialist and were diagnosed with neuropathic pain that was refractory to multiple treatment modalities. The majority of the patients presented with a history of trauma or a surgical procedure in the area before the onset of the pain. Preoperative examination included Tinel's sign and completion of the Visual Analog Scale (VAS) as an assessment of pain intensity. Inclusion criteria were: a clearly demarcated area of pain and positive Tinel's sign in the absence of an underlying cause such as a painful scar or neuroma as validated by means of high-resolution ultrasonography. ${ }^{17}$

\section{Surgical procedure}

Surgery was performed under general anesthesia. The donor site (usually abdomen) was infiltrated with saline $(\mathrm{NaCl} 0.9 \%)$ and $0.001 \mathrm{mg}$ adrenaline / $\mathrm{ml}$ before adipose tissue was harvested through a $3 \mathrm{~mm}$ blunt Mercedes tip cannula, at low suction settings using a Cytori pure graft system (Cytori Therapeutics Inc., San Diego, CA, USA). The lipoaspirate was then re-injected subcutaneously in the pre-operatively marked area using a fanning technique.

\section{Outcome measures}

Baseline characteristics were recorded. Primary outcome measures were patient satisfaction (yes or no) and pain scores pre- and post-operatively on the visual analogue scale (VAS) ranging from 0 'no pain at all', to 10 'unbearable pain'. A long-term follow-up was planned after a minimum of one year after surgery to evaluate changes in pain experience. Quality of sleep and safety of the procedure were also evaluated. 


\section{Statistical analysis}

Statistical comparison of the pre- and postoperative VAS scores was performed by paired Student's $t$-test. A p-value $<0,05$ was considered to be statistically significant.

\section{Results}

Fourteen individual patients were included. Their baseline characteristics can be found in table 1 . Thirteen patients (93\%) were satisfied with the treatment results at the first postoperative follow-up, while 11 patients $(86 \%)$ remained satisfied after more than one year follow-up. The mean VAS score was 7.4 before surgery (range 6-10) and significantly decreased to 3.8 after autologous fat grafting ( $p<0.0001$; range $0-8$; see figure 1$)$ at 8 weeks follow-up and 4.3 ( $p=0.0017$; range $0-10$ ) at long-term follow-up (on average 28 months). In 3 patients the VAS increased between the first and second follow-up, but was still below the pre-operative value. The quality of sleep improved in $50 \%$ of the patients, whereas the remainder indicated no difference. No complications were registered.

\begin{tabular}{|l|l|}
\hline Characteristics ( $\mathbf{n} \mathbf{1 4})$ & Mean (+ range) \\
\hline Age & $51(17-78)$ \\
\hline Gender (male/female) & $6 / 8$ \\
\hline BMI & $24.9(18.0-29.1)$ \\
\hline Smoking status (yes/no) & $4 / 10$ \\
\hline Duration of first follow-up & 8 weeks (2-20) \\
\hline Duration of second follow-up & 28 months (15-49) \\
\hline Total volume of fat injected & $21.8 \mathrm{ml}(2-70)$ \\
\hline Treated areas & \\
\hline Calcaneus & 1 \\
Achilles tendon & 1 \\
Malleolus & 1 \\
Patella & 4 \\
Upper leg & 4 \\
Dig IV & 1 \\
MCP & 1 \\
Infraorbital & 1 \\
\hline
\end{tabular}

Table 1. Patient Demographics 


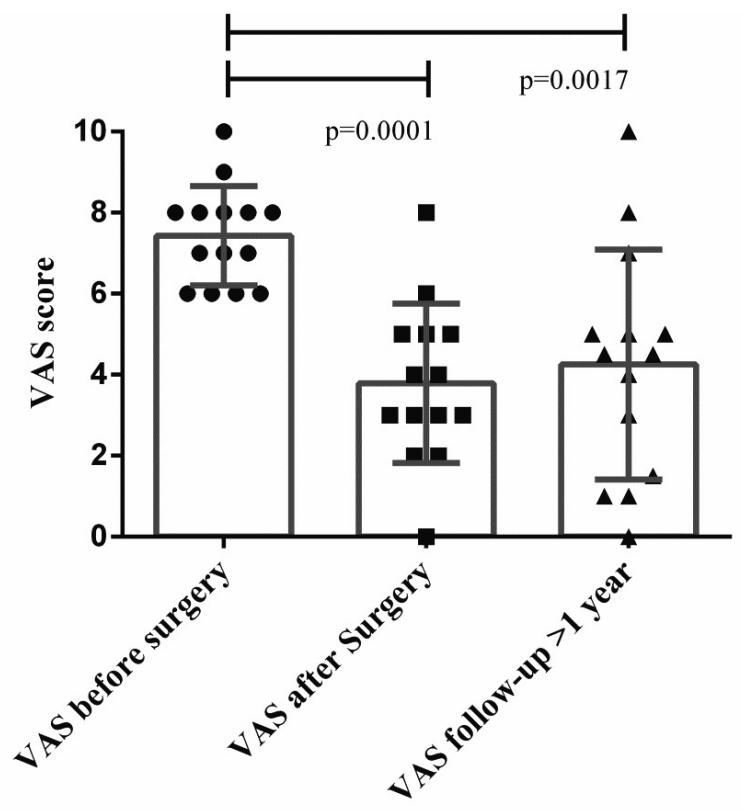

Fig 1. VAS score before surgery and at the two follow-ups. The mean VAS score significantly decreased from 7.4 (range 6-10) before surgery to 3.8 (range 0-8) after autologous fat grafting at 8 weeks follow-up and 4.3 (range 0-10) at the follow-up of more than one year. The VAS increased in 3 cases between the first and second follow-up, but was still below the pre-operative value.

\section{Discussion}

The study results revealed that the vast majority of patients $(86 \%)$ were satisfied with the pain relief after more than 1 year follow-up and $50 \%$ of the patients reported improved quality of sleep. In the literature, a pain reduction of at least $30 \%$ is generally regarded as being clinically relevant. ${ }^{3}$ This effect was reached in $9 / 14$ patients (64\%), while 5/14 patients (36\%) reported a pain reduction of at least $50 \%$. The meaningful effect of the procedure was corroborated by the fact that previous "standard care" pain treatments had either no or short-lasting effects, while the effect of autologous fat transfer lasted much longer.

The significant decrease in pain reflects the results from studies wherein cause of the pain was known. Vaienti et al reported a reduction of $23.2 \%$ in the mean disabilities of the arm, shoulder and hand (DASH) scores and an improvement in the VAS score of $22 \%$ (although not statistically significant) after perineural fat grafting in eight patients for the treatment of painful end-neuromas of the upper limb. ${ }^{10}$ Fredman et al noted a pain reduction in 6 out of 7 patients with burn scars. ${ }^{14}$ Huang et al reported a significant decrease in VAS score of 4.38 after one week, 5.38 after 4 weeks and 5.62 after 24 weeks of treatment, and a likewise 
decrease in the Neuropathic Pain Symptom Inventory in 13 patients with painful scars. ${ }^{16}$ In patients with PMPS several studies revealed that AFT significantly decreased the VAS score versus non-operated controls, with a mean decrease of $>3$ points. ${ }^{11,12,18}$

Interestingly, all fourteen patients reported pain relief in the first weeks after surgery. However, two patients indicated recurrence of pain after two months with an increased VAS score compared to pre-operative. Another patient reported at the long term follow-up that the VAS had been rising slightly after a few months, but was still at acceptable levels at the last follow-up. Although Huang et al found no recurrence of pain in patients responding to treatment, Vaienti et al described a similar deterioration of the pain relief over time in some patients. ${ }^{10,16}$ We hypothesize that graft volume loss, in literature described to be between $40-80 \%$ in the first months post-operative, may be responsible for the recurrence of pain. ${ }^{19-}$

${ }^{21}$ If that is the case, treatments or methods, which improve graft volume retention, may also improve the long term effects on pain.

\section{Conclusions}

This study shows that AFT might be a useful alternative in treating localized peripheral neuropathic pain. However, prospective controlled studies with larger study populations and control/sham groups are required to provide definitive evidence.

\section{Acknowledgements}

We would like to thank the department of anesthesiology of Maastricht University Medical Center and especially dr. Balthasar and dr. Sommer.

\section{References}

1. Smith BH, Torrance N. Epidemiology of neuropathic pain and its impact on quality of life. Current pain and headache reports. Jun 2012;16(3):191-198.

2. Yao C, Zhou X, Zhao B, Sun C, Poonit K, Yan H. Treatments of traumatic neuropathic pain: a systematic review. Oncotarget. Aug 22 2017;8(34):57670-57679.

3. Baron R, Binder A, Wasner G. Neuropathic pain: diagnosis, pathophysiological mechanisms, and treatment. The Lancet. Neurology. Aug 2010;9(8):807-819.

4. Stokvis A, van der Avoort DJ, van Neck JW, Hovius SE, Coert JH. Surgical management of neuroma pain: a prospective follow-up study. Pain. Dec 2010;151(3):862-869.

5. Dworkin $\mathrm{RH}, \mathrm{O}^{\prime}$ Connor $\mathrm{AB}$, Audette J, et al. Recommendations for the pharmacological management of neuropathic pain: an overview and literature update. Mayo Clinic proceedings. Mar 2010;85(3 Suppl):S3-14.

6. Treede RD, Jensen TS, Campbell JN, et al. Neuropathic pain: redefinition and a grading system for clinical and research purposes. Neurology. Apr 29 2008;70(18):1630-1635. 
7. Koch H, Hubmer M, Welkerling H, Sandner-Kiesling A, Scharnagl E. The treatment of painful neuroma on the lower extremity by resection and nerve stump transplantation into a vein. Foot \& ankle international. Jul 2004;25(7):476-481.

8. Balcin H, Erba P, Wettstein R, Schaefer DJ, Pierer G, Kalbermatten DF. A comparative study of two methods of surgical treatment for painful neuroma. The Journal of bone and joint surgery. British volume. Jun 2009;91(6):803-808.

9. Stahl S, Rosenberg N. Surgical treatment of painful neuroma in medial antebrachial cutaneous nerve. Annals of plastic surgery. Feb 2002;48(2):154-158; discussion 158-160.

10. Vaienti L, Merle M, Battiston B, Villani F, Gazzola R. Perineural fat grafting in the treatment of painful end-neuromas of the upper limb: a pilot study. The Journal of hand surgery, European volume. Jan 2013;38(1):36-42.

11. Caviggioli F, Maione L, Forcellini D, Klinger F, Klinger M. Autologous fat graft in postmastectomy pain syndrome. Plastic and reconstructive surgery. Aug 2011;128(2):349352.

12. Maione L, Vinci V, Caviggioli F, et al. Autologous fat graft in postmastectomy pain syndrome following breast conservative surgery and radiotherapy. Aesthetic plastic surgery. Jun 2014;38(3):528-532.

13. Ulrich D, Ulrich F, van Doorn L, Hovius S. Lipofilling of perineal and vaginal scars: a new method for improvement of pain after episiotomy and perineal laceration. Plastic and reconstructive surgery. Mar 2012;129(3):593e-594e.

14. Fredman R, Edkins RE, Hultman CS. Fat Grafting for Neuropathic Pain After Severe Burns. Annals of plastic surgery. Jun 2016;76 Suppl 4:S298-303.

15. de Gast H, Torrensma B, Fitzgerald E, Stevens H. The Treatment of Chronic Neuropathic Pain: Bio (Regenerative) Pain Treatment through Lipofilling. A Short Communication Case Series. Pain physician. Mar 2016;19(3):E495-498.

16. Huang $\mathrm{SH}, \mathrm{Wu} \mathrm{SH}$, Chang KP, et al. Alleviation of neuropathic scar pain using autologous fat grafting. Annals of plastic surgery. May 2015;74 Suppl 2:S99-104.

17. Toros T, Karabay N, Ozaksar K, Sugun TS, Kayalar M, Bal E. Evaluation of peripheral nerves of the upper limb with ultrasonography: a comparison of ultrasonographic examination and the intra-operative findings. The Journal of bone and joint surgery. British volume. Jun 2009;91(6):762-765.

18. Caviggioli F, Maione L, Klinger F, Lisa A, Klinger M. Autologous Fat Grafting Reduces Pain in Irradiated Breast: A Review of Our Experience. Stem cells international. 2016;2016:2527349.

19. Gir P, Brown SA, Oni G, Kashefi N, Mojallal A, Rohrich RJ. Fat grafting: evidence-based review on autologous fat harvesting, processing, reinjection, and storage. Plastic and reconstructive surgery. Jul 2012;130(1):249-258.

20. Liu B, Tan XY, Liu YP, et al. The adjuvant use of stromal vascular fraction and platelet-rich fibrin for autologous adipose tissue transplantation. Tissue engineering. Part $C$, Methods. Jan 2013;19(1):1-14.

21. Zhu $M$, Zhou $Z$, Chen $Y$, et al. Supplementation of fat grafts with adipose-derived regenerative cells improves long-term graft retention. Annals of plastic surgery. Feb 2010;64(2):222-228. 


\section{CHAPTER 4}

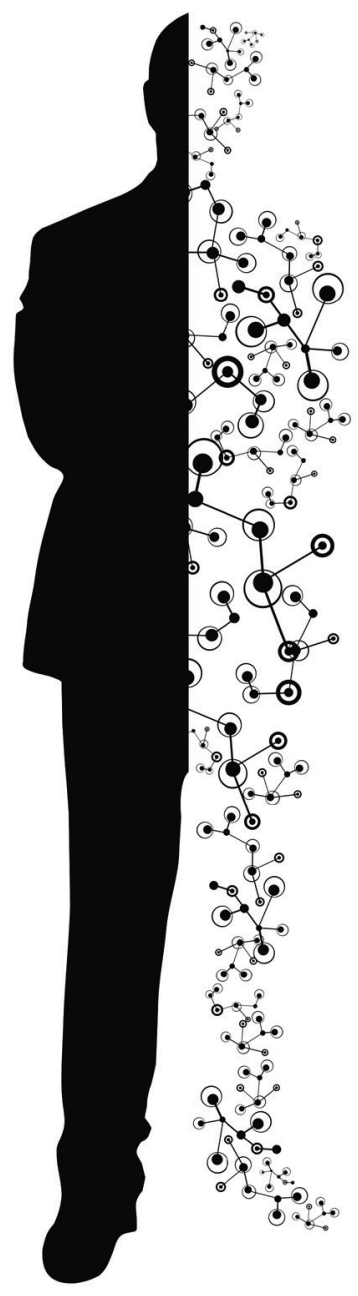




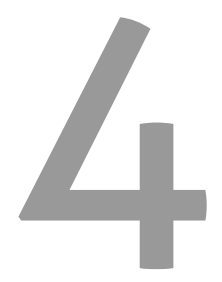

\section{Standardized human bone marrow- derived Stem Cells Infusion Improves Survival and Recovery in a rat model of Spinal Cord Injury}

Munter JP*, Beugels J*, Munter S, Jansen L, Cillero-Pastor B, Moskvin O, Brook G, Pavlov D, Strekalova T, Kramer BW, EC Wolters.

${ }^{*}$ contributed equally as first author 


\section{Abstract}

Spinal cord injury $(\mathrm{SCl})$ is an incurable disorder with an unmet need of an effective treatment. Recently, autologous human bone marrow-derived stem cells have shown to promote functional improvement, due to their anti-inflammatory and regenerative/apocrine properties. In this study, the primary objective was to test whether a single intrathecal injection with a $100 \mu \mathrm{L}$ suspension of 400,000 fresh human bone marrow-derived $\mathrm{CD}_{3} 4^{+}$and an equal number of $\mathrm{CD} 105^{+}$stem cells (Neuro-Cells (NC)), one day after balloon-compression of the spinal cord, improves motor function and reduces secondary damage in immunodeficient rats. During the first 5 weeks after this intervention, NC significantly improved locomotor recovery and induced less injury-associated adverse events compared to vehicle-treated rats.

Histological analysis showed that NC reduced astrogliosis, and apoptosis early after administration (day 4), but not at a later stage (day 56) after $\mathrm{SCl}$. Proteomic studies (at day 56) pointed to the release of paracrine factors and identified proteins involved in regenerative processes. As stem cells seem to reach their effects in acute lesions by mainly suppressing (secondary) inflammation, it is thus realistic to expect a lower magnitude of their eventual beneficial effect in T-cell deficient rats, a fact reinforcing the robustness of Neuro-Cells efficacy. Taken together, this study indicates that an intrathecal instillation of Neuro-Cells holds great promise as a neuro-regenerative intervention in a clinical setting with acute $\mathrm{SCl}$ patients.

\section{Introduction}

The prevalence of spinal cord injury $(\mathrm{SCl})$ worldwide is estimated at 2.5 million cases and the financial burden per case is calculated to be between 200,000 - 260,000 Euros/year (1). Pending severity of the injury, $\mathrm{SCls}$ may cause an (in)complete loss of sensory, motor and vegetative functions under the level of the spinal cord involved: in legs (paraplegia) or in arms and in legs (tetraplegia). Traditionally, in $60 \%$ of the cases, traumatic spinal cord injury is caused by falls and by motor vehicle accidents (2), but recently more and more combatwounded soldiers are affected (3). Due to the lack of disease-modifying interventions, life expectancy in $\mathrm{SCl}$ patients is impaired (4) and the quality of their lives is poor $(5,6)$. 
As a small functional improvement in $\mathrm{SCl}$ patients might come with a major increase in quality of life and daily independency, recently, much attention is given to control and reduce collateral damage of neural tissue by inhibiting the posttraumatic inflammatory cascades (7-10). Less than $10 \%$ of functional long-tract connections is needed to enable locomotion (11). Although this level of connectivity often remains after the injury, axons might become non-functional because of collateral damage resulting in neurodegeneration at a later stage (12). Therefore, prevention of secondary damage in the acute phase of spinal cord injury is of utmost importance, which offers the opportunity of functional improvement by disease-modifying interventions $(7,13)$.

Until the recent past, steroids were applied for this purpose (14), although wide spread use has stopped due to limited effects and severe adverse effects $(15,16)$. In SCl, a therapeutic intervention combining both anti-inflammatory and regenerative properties is an unmet need. Hypothetically, stem cells may be such an intervention, as one of the characteristics of adult stem cells (both hematopoietic and mesenchymal stem cells) is the ability to both inhibit inflammation and to mediate regeneration by increasing neuroplasticity in neurodegenerative processes $(8,17-25)$. Recent studies suggested that not only the site of the lesion, but also the timing of the administration of stem cells after the lesion are crucial for their beneficial effects in the context of the evolving post-traumatic inflammatory response (9,10,23-29). Dose and number of cells administrated appear to be of less importance (30).

The objective of this study was to investigate whether a single intrathecal administration with 'Neuro-Cells' (a not substantial manipulated low immunogenic, fresh human bone marrow-derived stem cell preparation, depleted of erythrocytes and the majority of the lymphocytes according to the manufacturing standard operating procedures of Neuroplast BV) provided a better survival and functional recovery in T-cell deficient rats after spinal cord injury.

In this study, a moderate grade $\mathrm{SCl}$ was applied in rats by a moderate balloon compression of a spinal thoracic (Th 9) segment. Unfortunately, treatment of these animals with autologous stem cells or with a low-immunogenic rat stem cell preparation (following the manufacturing procedure of Neuro-Cells) is not realistic due to the limited volume of rat bone marrow, and the invasiveness of the cell collection procedure itself in rats. Thus, T-cell deficient rats were selected to avoid both rejection of human stem cells and the use of 
immune suppressive drugs. Immune suppressive drugs are considered major confounders as they not only interfere with stem cells but also offer a cell protective effect to local neurons (31-34). They can substantially influence the outcome in experimental studies after the effects of stem cell interventions in favour of the vehicle treated animals. Therefore, these drugs dampen neuro-inflammation following acute spinal cord injury and thus final damage, with consequent less sensorimotor and neurovegetative symptoms (35). In comparison to normal, immune competent rats, the higher liability to infections may also have a significant effect on survival of the experimental animals (35).

We hypothesized that infusion with Neuro-Cells $24 \mathrm{~h}$ after $\mathrm{SCl}$ will improve locomotor recovery by reducing secondary damage. Functional behavioral recovery was monitored weekly using the Basso, Beattie, Bresnahan (BBB) open field test (36). Histological studies were performed 4 and 56 days post-surgery to determine the number of apoptotic cells, reactive astrocytes, and microglia infiltration in both vehicle and Neuro-Cells-treated animals. Moreover, we used mass-spectrometry technology to screen for and identify peptides in each site relative to the spinal lesion (rostral, lesion, caudal) obtained in vivo. We hypothesized that Neuro-Cells changes the composition of local peptides by down regulation of pro-inflammatory proteins as compared to the vehicle treated animals. This effect is expected to be the strongest in the lesion site as compared to the rostral and caudal sites

\section{Methods}

\section{Animals and experimental Design}

Adult male T-cell deficient A-thymic RH-Foxn1 ${ }^{\text {rnu }}$ (260-310 g) purchased from Harlan (Harlan laboratories, the Netherlands) were housed in pairs during the acclimatization period, for post-operative care and throughout the rest of the experiments. Rats were maintained in an isolated part of the animal facility apart from immune competent animals and under standard housing conditions (12 h light/dark cycle, lights on at 8:00 a.m., humidity 40-60\%, temperature $22 \pm 1{ }^{\circ} \mathrm{C}$ ) with ad libitum access to food and water. The Animal Care and Use Committee of the University of Maastricht reviewed and approved all animal surgeries, procedures, and post-operational care (permit number DEC2013-013). Laboratory personnel managed animals following the National Institute of Health Guide for the Care and Use of 
Laboratory Animals. A total number of 62 rats underwent surgical procedures for inclusion in this study (Figure 1).

\section{Transplantation}

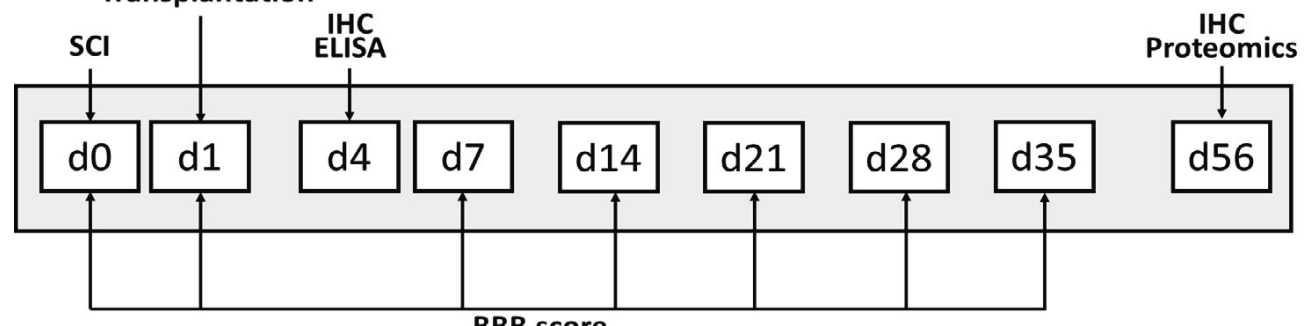

BBB score

Figure 1: Experimental design. At day $0(\mathrm{dO})$, spinal cord injury $(\mathrm{SCl})$ or a sham lesion was induced in $T$-cell deficient rats. Rats were injected intrathecal with vehicle or Neuro-Cells, caudal to the lesion at day 1. Immunohistochemistry (IHC) was performed at day 4 and day 56 after administration. Proteomic studies were performed at day 56. Throughout the study, Basso-Beattie-Bresnahan (BBB) measurements for assessing locomotor behaviour (36) were performed on a weekly base.

\section{Spinal Cord Balloon Compression}

After a subcutaneous injection of buprenorphine $(0.05 \mathrm{mg} / \mathrm{kg})$, animals were anaesthetized (3-4\% Isoflurane) and maintained (1.5-2.5\% Isoflurane, Sigma-Aldrich) in anesthesia with a stable body temperature of $37^{\circ} \mathrm{C}$. Having removed the spinal processes T10-T11 after a 2-cm midline incision at T10-L1, a small hole (1.5 mm diameter) was drilled in the vertebral arch T10, using a surgical microscope (37). After opening the periosteal membrane in order to allow direct visualization of the spinal cord with intact dura mater, a groove was drilled in the midline on the dorsal surface of the vertebral T11 lamina. Then, an epidural inserted French Fogarty catheter (Baxter Healthcare Corporation, Irvine, CA) filled with saline and connected to an airtight $50 \mu \mathrm{L}$ Hamilton syringe type 1705 , cranially for $1 \mathrm{~cm}$, was guided through this groove, thus positioning the center of the balloon at T8-T9. In case of SCllesioning, but not in sham-lesioning, the balloon was then rapidly inflated with $15 \mu \mathrm{L}$ saline and held in situ during 5 minutes before deflating and removal of the catheter, closing the wound and terminating anesthesia. Post-operative care included subcutaneous application of buprenorphine $0.05 \mathrm{mg} / \mathrm{kg}$ during 4 days to relief the pain. 


\section{Neuro-Cells}

Neuro-Cells is the working name (patent W02015/059300A1) of a fresh, standardized human bone marrow derived stem cells containing product, that is produced under good manufacturing practices (GMPs) without expansion and/or labeling (or other major manipulation) of the stem cells. Neuro-Cells comprises of hematopoietic stem cells and their progenitors (HSCs), mesenchymal stem cells and their progenitors (MSCs) and other mononuclear cells. Four healthy male volunteer donors were recruited with informed consent for collection of 50-75 mL bone marrow from their iliac crest under local anesthesia, following standard operating procedures. Neuro-Cells was depleted from erythrocytes by Ficoll (GE Healthcare, Chicago, III.) density gradient centrifugation (at 400g, at room temperature) and the number of lymphocytes was substantially reduced (table 1 ) by positive selection between 95-97\%, and resuspending the remaining cells in Ringer-lactate. MSCs and HSCs were characterized by flow cytometry (Miltenyi, Germany). The group of MSCs were identify by being positive for CD73, or CD90, or CD105 and negative for CD34 and CD45 and CD14 (38). HSCs were positive for CD34, and CD38. Every Neuro-Cells batch fulfilled the GMP release criteria of at least 4 million HSCs and an equal number of MSCs suspended in $10 \mathrm{~mL}$ Ringer-lactate for intrathecal application. Besides the intra-donor variation, the intrathecal treatment of the SCl-rats was calibrated to $4.0 \times 10^{5} \mathrm{CD} 34^{+}$cells in vials of $100 \mu \mathrm{L}$. However, due to the fresh nature of the cells and the calibration based on the number of $\mathrm{CD}_{3} 4^{+}$cells, the individual number of MSCs will differ per used donor. Table 1 gives an overview of the number of cells applied to the individual rats.

\begin{tabular}{|c|c|}
\hline Cell population in Neuro-Cells & $\begin{array}{c}\text { Mean absolute number of cells injected in each animal } \\
\text { (in 100 } \mathbf{\mu l} \text { ) }\end{array}$ \\
\hline Total nucleated cells & $1.2 \times 10^{7}$ \\
\hline $\mathrm{HSC}:$ total CD34 ${ }^{+}$cells & $4.0 \times 10^{5}$ \\
\hline MSC: $\mathrm{CD} 271^{+}$cells & $7.8 \times 10^{4} \pm 2.6 \times 10^{4}$ \\
\hline MSC: $\mathrm{CD} 133^{+}$cells & $1.5 \times 10^{4} \pm 0.5 \times 10^{4}$ \\
\hline MSC: $\mathrm{CD} 90^{+}$cells & $3.7 \times 10^{3} \pm 1.3 \times 10^{3}$ \\
\hline MSC: $\mathrm{CD} 105^{+}$cells & $3.5 \times 10^{5} \pm 2.0 \times 10^{5}$ \\
\hline MSC: $\mathrm{CD} 73^{+}$cells & $4.5 \times 10^{3} \pm 2.1 \times 10^{3}$ \\
\hline
\end{tabular}

Table 1: Characterization of cell population in 'Neuro-Cells'.

\section{Intrathecal Infusion}

At day 1 post-surgery, we subcutaneously injected the rats with $0.05 \mathrm{mg} / \mathrm{kg}$ buprenorphine (AST Farma B.V., The Netherlands) and further anaesthetized them with isoflurane 
(induction phase with 3-4\% isoflurane and maintenance phase with $1.5-2.5 \%$ isoflurane), keeping the rats' body temperature at $37^{\circ} \mathrm{C}$ using heating pads. The wound of the first operation was reopened in order to visualize the dura. Then the dura was punctured and, after collection of a drop of cerebrospinal fluid (CSF), a syringe, either filled with Neuro-Cells or vehicle (Ringer lactate), was connected and emptied, caudally of the lesion, through a microliter pump over a time period of 5 minutes. We infused SCl-treated rats with $100 \mu \mathrm{L}$ Neuro-Cells or $100 \mu \mathrm{L}$ vehicle. Post-operative treatment and care were identical to those after the lesioning. Local pressure at the puncture hole and applying some drops of the animal's blood on the puncture hole prevented for CSF leakage.

\section{Post-operative Care}

Animals' wellbeing, general health condition, urodynamic and body weights were assessed twice per day until day 35. The bladder was emptied manually twice a day until the rats were able to spontaneously empty their bladder again, usually within 14 days after the lesion. During the period of manual bladder emptying, the urine was routinely checked for bacteria growth using a urine dipstick.

\section{Functional Evaluation}

Rats were assessed for locomotor behavior with the Basso-Beattie-Bresnahan (BBB) open field test (36). Locomotor recovery was tested pre-SCI (baseline) and at days 1, 7, 14, 21, 28 and 35 post-lesion. At post-surgery day 1, SCl-lesioned rats had to display a flaccid paraplegia (BBB score 0) before the application of Neuro-Cells or vehicle. Based on the established natural recovery, $\mathrm{SCl}$-lesioned animals were also excluded when scoring was below 4 (protracted recovery) and/or above 8 (partial lesion) at day 7 (37). The exclusion parameters of animals were established before the execution of the experiment. Open field locomotor function was assessed by two independent researchers, evaluating videotapes not aware of the treatment.

\section{Immunohistochemical Analyses}

At days 4 and 56, four rats out of the NC- and Veh.-treated SCl animals and three out of the NC- and Veh.-treated Sham animals were anesthetized with isoflurane (induction phase with 
$3-4 \%$ isoflurane and maintenance phase with $1.5-2.5 \%$ isoflurane) and sacrificed by transcardial perfusion with Ringer lactate (Baxter, $\mathrm{pH}$ 7.4). The spinal cord tissue of the entire affected area was positioned into cryo-molds in a longitudinal orientation and embedded in optimal cutting temperature compound (OCT, VWR, The Netherlands; for rats sacrificed 4 days post $\mathrm{SCl}$ ) or porcine gelatin (10\%, Sigma Aldrich; for rats sacrificed 56 days post $\mathrm{SCl}$ ). Subsequently, the spinal cord tissue was snap frozen in liquid nitrogen and stored at $-80^{\circ} \mathrm{C}$ until further processing. To assess the presence of human-derived transplanted cells, as well as astrogliosis, inflammation and apoptosis of the infused cells, serial longitudinal cryosections of the spinal cord $(10 \mu \mathrm{m})$ were cut through the areas of interest: the rostral part of the lesion (RO), the center of the lesion (CE), and the caudal part of the lesion (CA), using a cryostat (Leica Biosystems), and then stored at $-20^{\circ} \mathrm{C}$ until further processing. To identify human stem cells transplanted into the rat spinal cord at day 4 after infusion caudally to the lesion, antibodies directed against human mitochondrial (MAB1273C3, 1:100, clone 113-1, Millipore) were used. To study the early- and late-effects of Neuro-Cells in inflammation, astrogliosis and apoptosis, sections were incubated overnight at $4^{\circ} \mathrm{C}$ with the following primary antibodies: rabbit anti-CD68 (Abcam, 1:500, ab125212), rabbit anti-GFAP (Abcam; 1:500, ab7260), and rabbit anti-cleaved caspase-3 (Cell signaling, 1:500, [Asp175] 9661). Sections were washed with 1 x PBS and incubated with secondary antibodies [(donkey anti-rabbit Alexa 488 (Invitrogen, 1:100) or donkey antimouse Alexa 488 (Invitrogen, 1:100)] in 0,1\% blocking buffer for 1 hour at room temperature. Cell nuclei were stained for 10 minutes with 4',6-diamidino-2-phenylindole (DAPI, Thermofisher).

For quantitation, two sections per rat were visualized with a confocal microscope (DSU, Olympus $^{\circledR}$ BX51W1; 20x objective) and Stereolnvestigator software (MicroBrightField, Williston, VT) and ImageJ programs (NIH, Bethesda, MD) were used for analysis. Image J was used to calculate the area of each anatomical region (rostral, lesion and caudal) and integrated mean density. The corrected total cell fluorescence (CTCF) of GFAP ${ }^{+}$and $C D 68^{+}$ was calculated as integrated density - (area of selected region $\mathrm{x}$ mean fluorescent intensity of background reading). For the CA and RO sites of the lesion, fluorescence intensity was measured at the outer surface of the spinal cord and at the middle part of the section (2 points at each part of the lesion (S1, Figure 1). For the CE part, fluorescence intensity was 
measured at the border of the center lesion. The average values of fluorescence intensities from 2 images were used as the reference for comparisons within each group.

Serum of the rats sacrificed at day 4 was taken for ELISA testing to determine the concentration of interleukins and compare the concentrations of the vehicle and Neuro-Cells treated $\mathrm{SCl}$-lesioned animals with the sham lesioned animals. To study the concentrations of IL-1 $\beta$, IL-6 and TNF $\alpha$ in a blood plasma, rat enzyme-linked immunosorbent assay (ELISA) was performed using Rat Interleukin 1 beta (Rt IL-1ß) ELISA kit (ThermoFisher Scientific, Waltham, MA, USA), IL-6 Rat ELISA Kit (ThermoFisher Scientific, Waltham, MA, USA) and TNF $\alpha$ (Rt TNF $\alpha$ ) Rat ELISA Kit (ThermoFisher Scientific, Waltham, MA, USA) according to the manufacturer's instructions. The microwell absorbance was measured at $450 \mathrm{~nm}$ with Promega microplate reader for all cytokines (Promega, Madison, WI, USA).

\section{Statistical Analysis}

Analyses were performed using statistical package SPSS 17.0 for Windows XP. Data were normally distributed as determined by Shapiro-Wilk tests for normality and we therefore performed parametrical statistical analyses on datasets. We performed a two-way ANOVA (with 'group' as the predicting factor and 'lesion site' as the moderating factor) to assess the data obtained from the histology study. If a group effect, level on spinal cord effect or an interaction effect (group $x$ lesion site) was significant a post hoc Fishers Least Significant Difference (LSD) test was performed, after determining equality of variances using Levene's test (which was the case unless stated otherwise). Repeated measures ANOVA was used to analyze the BBB score and the bladder recovery function over the different days with withinsubjects factor "testing day", and "group" as fixed between-subject factors. Huynh-Feldt correction was used to correct for violations of sphericity. Rat survival as a function of treatment was determined as Kaplan-Meier estimates, and differences in the survival curves were evaluated with this method. There were no censored data. A $p$ value $\leq 0.05$ was considered significant. Data are presented as mean with standard error of the mean (SEM). 


\section{Proteomics}

\section{Materials}

For proteomic studies in NC-treated and Veh-treated rats, sacrificed at day 56, ammonium bicarbonate, dithiothreitol, iodoacetamide and trifluoroacetic acid (ULC grade) were purchased from Sigma-Aldrich, urea from GE Healthcare, the enzyme mix trypsin/lysC (mass spec grade) from Promega, and water, acetonitrile, formic acid, all ULC grade, from Biosolve. Sample Preparation

Gelatin was removed from the spinal cords by 3 washes in warm $\left(30-35^{\circ} \mathrm{C}\right) 50 \mathrm{mM}$ Ammonium bicarbonate ( $A B C)$. After washing, $5 \mathrm{M}$ Urea in $50 \mathrm{mM} A B C$ was added to the spinal cord tissues. Tissue disruption and lysis was performed by three freeze-thaw cycles using a warm water bath and liquid nitrogen. During 45 minutes, the lysate then was reduced with $20 \mathrm{mM}$ Dithiothreitol (DTT) before being alkylated with $40 \mathrm{mM}$ lodoacetamide (IAM) for another 45 minutes in the darkness. The alkylation was terminated by 20mM DTT to consume any excess IAM. Digestion was performed with a mixture of LysC and Trypsin, which was added at a ratio of 1:25 (enzyme to protein). After two hours of digestion at $37^{\circ} \mathrm{C}$ in a water bath, the lysate was diluted with $50 \mathrm{mM} \mathrm{ABC}$ to $1 \mathrm{M}$ Urea and further digested at $37^{\circ} \mathrm{C}$ overnight. The digestion was terminated by addition of formic acid (FA) to a total of $1 \%$. Biognosys iRT's were added to each peptide sample according to manufacturer's instructions (required for the DIA analysis using Spectronaut X software, Biognosys Inc., Beverly, MA, USA).

Liquid Chromatography - Mass Spectrometry

Peptide separation was performed on a Thermo Scientific (Dionex) Ultimate 3000 Rapid Separation UHPLC system equipped with an Acclaim PepMap C18 analytical column ( $2 \mu \mathrm{m}$, $100 \AA ̊, 75 \mu \mathrm{m} \times 150 \mathrm{~mm}$ ). Peptide samples were first desalted on an online installed C18 trapping column. Desalted peptides were then separated on the analytical column with a 90 minutes linear gradient from $5 \%$ to $35 \%$ Acetonitrile (ACN) with $0.1 \%$ FA at $300 \mathrm{~nL} / \mathrm{min}$ flow rate. The UHPLC system was coupled to a Q Exactive HF mass spectrometer (Thermo Scientific). DDA settings were as follows. Full MS scan between $375-1,500 \mathrm{~m} / \mathrm{z}$ at resolution of 120,000 followed by MS/MS scans of the top 15 most intense ions at a resolution of 15,000. The HRM DIA (data-independent acquisition) method consisted of a survey full MS 
scan at 120,000 resolution at $350-1,650 \mathrm{~m} / \mathrm{z}$. Then 58 DIA windows were acquired at 30,000 resolution $(\mathrm{S} 2$, Table 1$)$.

\section{Data Analyses}

For protein identification the DDA spectra were analyzed with Proteome Discoverer (PD) version 2.1.1.21. Within the PD software, the search engine Sequest was used with the SwissProt Human (Homo sapiens (TaxID=9606) (v2016-11-30)) and Rat (Rattus norvegicus (TaxID=10116) (v2016-11-30) databases and the Biognosys iRT peptide sequences (supplied by Biognosys). The database search was performed with the following settings: enzyme was trypsin, a maximum of 2 missed cleavages, minimum peptide length of 6 , precursor mass tolerance of $10 \mathrm{ppm}$, fragment mass tolerance of $0.02 \mathrm{Da}$, dynamic modifications of methionine oxidation and protein $\mathrm{N}$-terminus acetylation, static modification of cysteine carbamidomethylation. The DDA measurements were used to create a spectral library using spectral library generation in Spectronaut 9 (39) (Biognosys). Only identifications with FDR of maximum $1 \%$ at peptide and protein level were taken into account for spectral library generation. For protein quantitation, the DIA data were analyzed with Spectronaut 9, with the manufacturer's recommended default settings.

\section{Differential abundance testing}

Within each site of the lesion (RO, CE, CA), differential protein abundance between NeuroCells- and vehicle-treated conditions was tested using Spectonaut's built-in algorithm. Abundance data for proteins found differentially abundant with q-value below 0.05 was submitted to downstream functional analysis.

\section{Representations of biological functionality}

The following complementary knowledge-based classifications of protein functionality were used in combination, to create biological interpretation of the detected lists of differentially abundant proteins: i) Gene Ontology (including Biological Process and Cellular Component branches of the hierarchy) were adopted from GSEA website of Broad Institute (40) ii) regulons, generated by collecting the known transcription-target relationships from HTRIdb (41) and CellNet (42) and iii) Reactome pathways (43). For all the functionality representations, members of the gene ID-based categories were remapped to protein IDs 
with Bioconductor's biomaRt package (44). The sets of protein IDs were restricted to proteins actually detected in the experiment (at any lesion site and condition).

\section{Protein set enrichment analysis}

Functionality enrichment tests with lists of responsive proteins (40) in context of the 3 functionality representations described above, were performed with goseq package (45) using Wallenius approximation and correction for protein length. Statistical significance of the enrichment tests was estimated with 100,000 data permutations.

\section{Protein-sharing networks}

To identify response patterns at a general level rather than individual functional categories, we generated protein-sharing networks. For this purpose, we collected respective categoryprotein relationships (e.g. Reactome pathways) and restricted them to proteins that were called responsive to Neuro-Cells in at least one of the three sites. Subsequently, for each pair of the categories (network nodes), the number of shared proteins was recorded and used to map to the width of the edge connecting those two nodes. The analysis was performed with a combination of $\mathrm{R}$ programming language ( $\mathrm{R}$ core team, 2014) and Cytoscape network analysis platform (46) version 3.6.1.

\section{Results}

\section{Animals}

During the quarantine period, 66 rats were randomized into two groups: group $1(N=49)$ for $\mathrm{SCl}-$ lesioning, and group $2(\mathrm{~N}=17)$ for sham-lesioning. Before this lesioning, though, one animal belonging to group 2 was lost because of intercurrent health problems, and during the $\mathrm{SCl}$-lesioning another one (out of group 1), because of anaesthesia-related death. The 48 surviving $\mathrm{SCl}$-lesioned animals in group 1 were further randomized in 24 rats for treatment with Neuro-Cells (SCl-NC animals) and 24 rats for treatment with the Vehicle (SCl-Veh animals). The 16 surviving sham-lesioned rats were randomized into 7 animals for a treatment with Neuro-Cells (Sham-NC animals) and 9 animals for the intervention with the Vehicle (Sham-Veh animals). All SCI-lesioned animals had a BBB score of 0 points before the 
application of their treatment. Day 4 of the study, $4 \mathrm{SCl}-\mathrm{NC}$ and $4 \mathrm{SCl}-$ Veh as well as 3 ShamNC and 3 Sham-Veh. animals were sacrificed for immunohistochemistry. Within the first week after the $\mathrm{SCl}$-lesioning, 13 animals had to be excluded from the experimental design: 2 $\mathrm{SCl}-\mathrm{NC}$ animals (due to direct perilesional surgical complications and shock) and $3 \mathrm{SCl}-\mathrm{Veh}$ animals (due to direct post-lesional convulsions, shock, and artificial-induced bladder rupture). Conform the protocol, another $4 \mathrm{SCI}-\mathrm{NC}$ rats had to be excluded because of a BBBscore at day $7<4$ (probably because of a histological confirmed increase of the SCl-lesion due to the intrathecal injection of the viscous Neuro-Cells preparation), and $4 \mathrm{SCl}$-Veh rats because of a BBB-score $>8$ (probably because of an impartial $\mathrm{SCl}$ lesion). During the study, 1 $\mathrm{SCl}-\mathrm{NC}$ rat and $5 \mathrm{SCl}-\mathrm{Veh}$ animals reached a humane endpoint due to $\mathrm{SCl}$-surgery-induced complications. The 9 Sham-Veh and 7 Sham-NC treated animals, during the study, did not suffer any surgery-related complication, nor did they reach any humane endpoint. So, in the end $13 \mathrm{SCl}-\mathrm{NC}$, $8 \mathrm{SCl}-\mathrm{Veh}, 9$ Sham-Veh, and 7 Sham-NC treated rats completed the study, and were sacrificed at day 56. An overview of the study is displayed in Fig. 2.

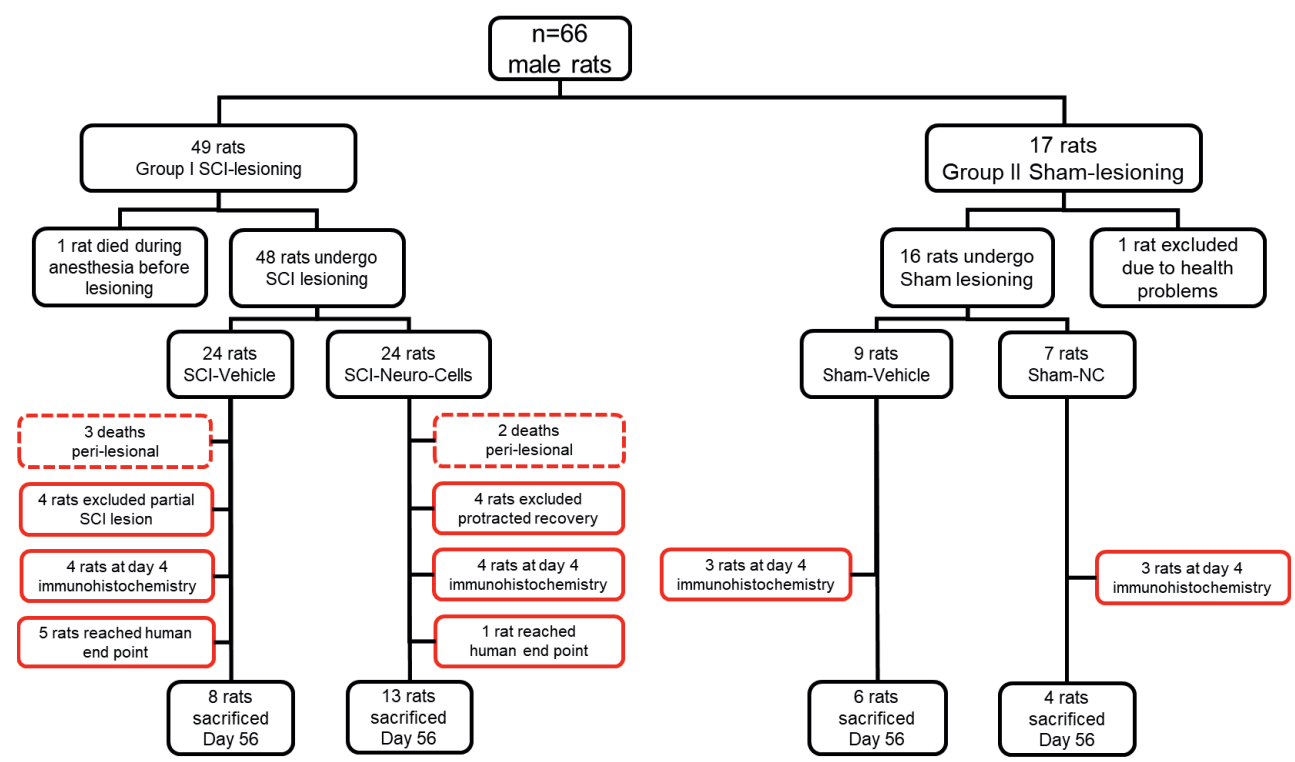

Figure 2: Flowchart with the number of rats used in this study. Red squares represent animals excluded from the study as described in the text.

\section{Survival}

There were no mortalities in the 16 sham-lesioned animals. In the SCl-lesioned animals, apart from the 5 peri-lesional direct $\mathrm{SCl}$-surgery related deaths, during the 56 days following 
lesioning, 5 out of 13 Vehicle-treated animals reached a humane endpoint, due to development of ascites ( $n=3$; day4, day5, day6), kidney failure $(n=1 ;$ day18) and severe body weight loss ( $n=1$; day38). In Neuro-Cells treated rats only 1 out of 14 animals reached a humane endpoint at day 10 post-surgery, due to severe body weight loss (Figure 2). The SCllesioned rats treated with Neuro-Cells appear to survive longer, as group differences reached significance $(p \leq 0.05)$. A plot of the survival function is shown in Figure 3.

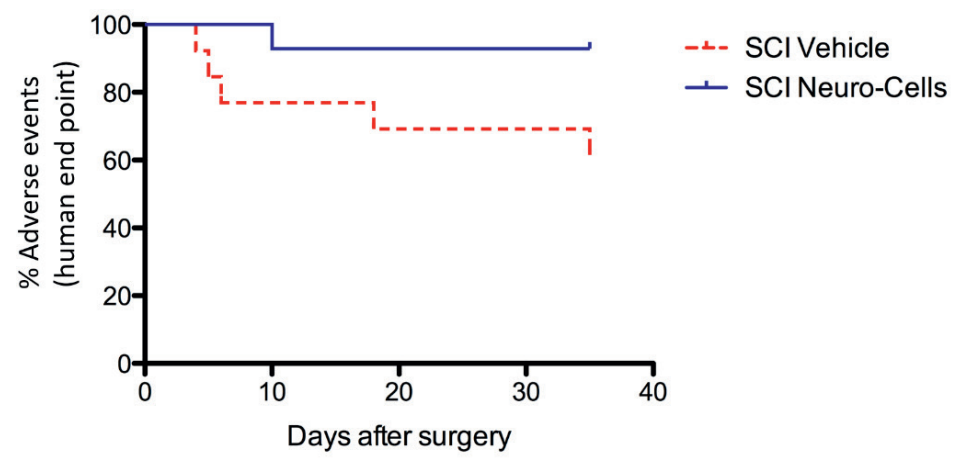

Figure 3: Kaplan-Meier estimates of postoperative survival (adverse events reaching human end point) stratified by treatment with either vehicle or Neuro-Cells. Rats of the SCl-Neuro-Cells group survive longer $(p \leq 0.05)$.

\section{Adverse events, general health conditions, body weight and urodynamic functions}

There were no mortalities in the 16 sham-lesioned animals. During the 56 days following lesioning, 5 out of 13 Vehicle-treated animals reached serious adverse events (SAEs) with a humane endpoint, due to development of ascites ( $n=3$; day4, day5, day6), kidney failure ( $n=1$; day18) and severe body weight loss ( $n=1 ;$ day38). In Neuro-Cells treated rats only 1 out of 14 animals reached such SAE at day 10 post-surgery, due to severe body weight loss (Figure 2). The $\mathrm{SCl}$-lesioned rats treated with Neuro-Cells appear to suffer significant less SAEs $(p \leq 0.05)$. A plot of the survival function is shown in Figure 3. Apart from these SAEs, surviving animals, suffered a higher incidence in the NC-treated group $(p=0.12)$ of a temporary cystitis, favorably reacting to a treatment with Baycal.

All surviving animals, after a modest loss of body weight during the first week after surgery, did gain body weight during the following weeks of the study period. Animals that underwent spinal cord injury and that were treated with Neuro-Cells did not differ in body weight compared to vehicle-treated group at day 1 and day 35 post-surgery (S5, table 4). 
Sham-lesioned animals, whether treated with Neuro-Cells or Vehicle, did not suffer any adverse event, loss of body weight, or any loss of urodynamic functions.

Regarding the $\mathrm{SCl}$-related loss of urodynamic functions, after surgery, all animals needed assistance with bladder voiding. Measuring manually expelled urine, NC-treatment did not affect the recovery of bladder control throughout a 14 day observation period (repeatedmeasurement ANOVA, $p=0.916$ ) as compared to the Vehicle-treated animals (see S6, Figure 3).

\section{BBB Score}

On day 1, the sham-lesioned groups received the highest BBB score possible, whereas both the $\mathrm{SCl}$-treated groups scored the lowest possible (0 points), demonstrating the validity of this rat model of $\mathrm{SCl}$. The scores increased in both $\mathrm{SCl}$-vehicle and $\mathrm{SCl}-\mathrm{Neuro}$-Cells groups over time (repeated measure ANOVA, $F(6,114)=2.72, p<0.032$ ). However, by 14 days and 35 days post-surgery, there was a statistically significant difference in BBB scores between the Neuro-Cells- and vehicle-treated rats $(p<0.01 ; p<0.008$ respectively; Figure 4$)$. These results indicated that Neuro-Cells induced functional improvement after $\mathrm{SCl}$ in rats.

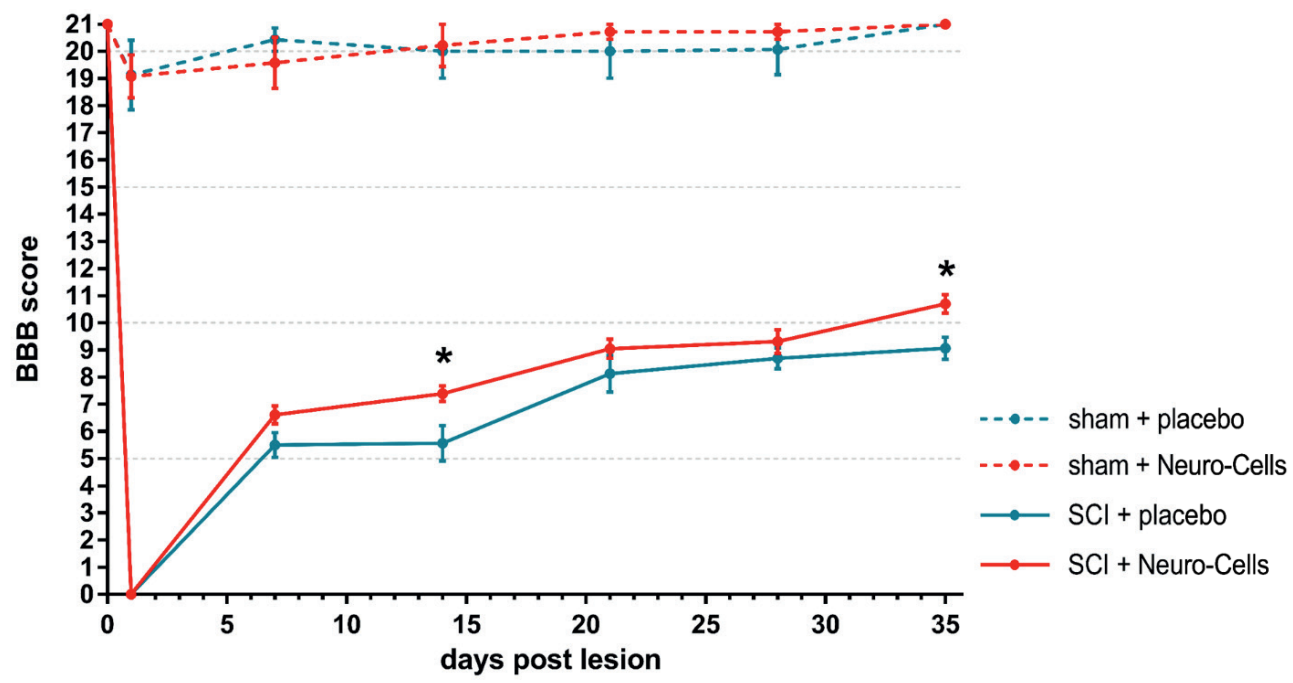

Figure 4: Effects of Neuro-Cells and Vehicle (placebo) on locomotor function in rats after SCl-surgery and sham $\mathrm{SCl}$ surgery. The first $\mathrm{BBB}$ evaluation took place at day 0 , before surgery. At that time, no statistically significant differences in BBB scores were found between NC and Veh treated SCI-rats, whereas the BBB scores in the sham-operated rats during the whole study were different from $\mathrm{SCl}$ operated rats $(p<0.001)$. During the whole study, BBB scores in NC-treated SCl-rats were higher as those in Veh-treated SCl-rats, differences reaching significance at days 14 and $35\left({ }^{*} p<0.01\right)$. 


\section{ELISA-serum}

The concentration of serum interleukin-1b, 6 and TNF-a were measured by ELISA. Figure 5 compared the mean differences between the vehicle respectively Neuro-Cells treated animals with the Vehicle and Neuro-Cells treated sham lesioned animals.
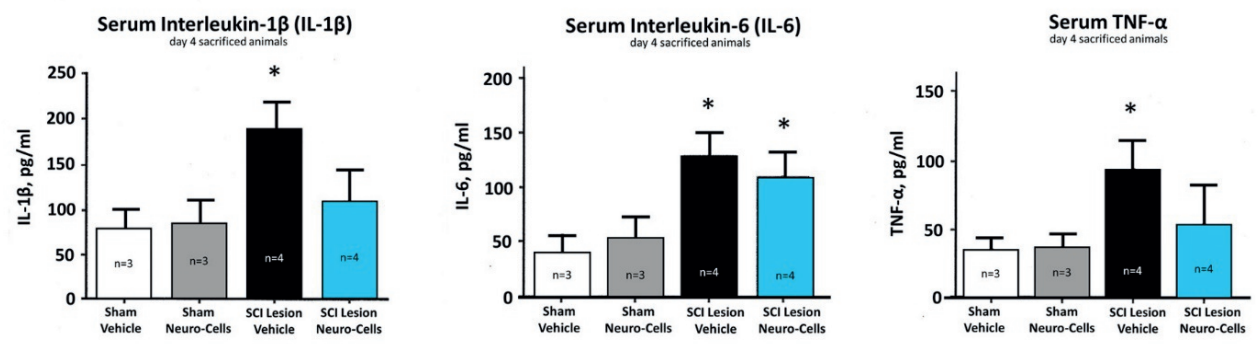

Figure 5: Differences in serum concentration between the Veh.- and NC-treated SCl-lesioned and sham-lesioned animals at day 4 . At that time, there was a significant increase of serum Interleukin-1 $\beta$ and serum TNF- $\alpha$ in the Veh.-treated SCl-lesioned animals compared to the Veh.- and NC-treated sham-lesioned animals, but not for the NC-treated groups $\left({ }^{*} p<0.05\right)$. The serum Interleukin- 6 was lower in the $\mathrm{NC}$ treated SCl lesioned animals as compared to the Veh.-treated SCl-lesioned animals but both were significantly higher as compared to their sham-lesioned counterpart.

\section{Immunohistochemistry}

The survival of human transplanted stem cells was evaluated 3 days post-implantation of Neuro-Cells. Human positive cells were found partially attached to the spinal cord surface, which were identified by human mitochondrial epitopes (Figure 6B and 6C).
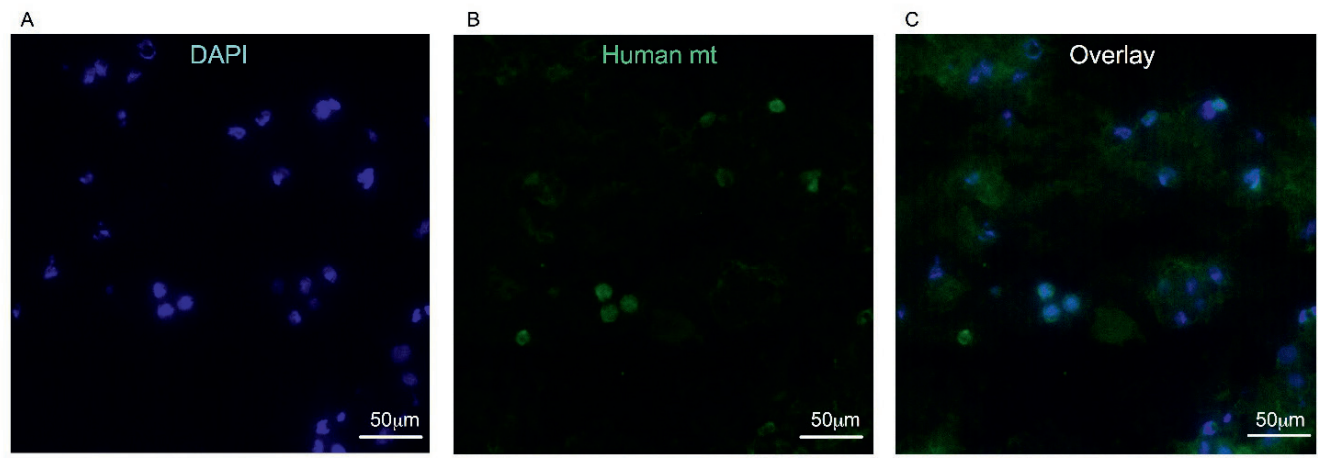

Figure 6: Presence of human cells at $\mathrm{CA}$ (and less in $\mathrm{CE}$ ) in NC-treated $\mathrm{SCl}$ rats detected by human mitochondrial epitopes, displayed in representative images at $\mathrm{CA}, 3$ days after the treatment. A) DAPI, B) Human $\mathrm{mt}, \mathrm{C}$ ) Overlay. Scale bars represents $50 \mu \mathrm{m}$. 
Early effects (day 4) on post-injury cellular responses

Monocytes and macrophages are key players of the inflammatory modulation and of the resolution of inflammation after $\mathrm{SCl}(47,48)$. In comparison to sham lesioned, vehicle or NCtreated animals, we observed a significant effect at the $\mathrm{SCl}$-induced lesion site ( $\mathrm{F}(1$, 42) $=56.02, p<0.001)$ in $C D 68^{+}$expression at day 4 , with an increase at the central part of the lesion. Post hoc analysis showed that the $\mathrm{CD}^{+} 8^{+}$expression was significantly increased $(p<0.008)$ at the CA in the Neuro-Cells-treated rats compared to vehicle-treated rats (Figure 7B). No differences were found in the $\mathrm{CD} 8^{+}$expression at the RO between the two treated groups (Figure 7B).

$\mathrm{SCl}$-induced astrogliosis is an important process in glial scar tissue formation, as reactive astrocytes increase their expression of GFAP. In order to assess astrogliosis in $\mathrm{SCl}$ rats (vehicle vs. Neuro-Cells) 4 days post-injury, we examined the GFAP expression at the RO, CE and CA site of the lesion, in relation to those in the sham lesioned, vehicle or NC-treated animals (Figures 6C and 7D). Analysis showed a significant main effect of the SCl-induced lesion site in GFAP expression $(F(1,42)=29.05, p<0.001)$ with an increase in $\mathrm{GFAP}^{+}$expression at the central part of the lesion. There was also a significant main effect of the group ( $F$ $(1,42)=4.61, p<0.038)$, as Neuro-Cells treated rats had an overall lower number of GFAP ${ }^{+}$ expression as compared to the vehicle-treated group of rats. Post hoc analysis revealed a significant decrease in $\mathrm{GFAP}^{+}$expression at the CE in the Neuro-Cells-treated rats $(p<0.042$; Figure 7D) when compared to vehicle-treated rats. No differences were found in the GFAP ${ }^{+}$ expression between the Neuro-Cells- and vehicle-treated groups at the RO or the CA site of the lesion (Figure 7D).

Components of the caspase- 3 apoptotic pathway are activated after traumatic $\mathrm{SCl}$ in rats and occur early in neurons at the injury site and hours to days later in oligodendroglia adjacent to and distant from the injury site $(49,50)$. To examine the effects of vehicle and Neuro-Cells on apoptotic cell death after $\mathrm{SCl}$, we quantified the expression of cleaved caspase $-3^{+}$at day 4 post-injury in relation to those in sham-lesioned, vehicle or NC-treated rats (Figures 7E and 7F). We found a significant effect in the $\mathrm{SCl}$-lesion site $(\mathrm{F}(1,42)=9.30$, $p<0.001)$, namely an increased number of cleaved caspase $-3^{+}$cells at the lesion site and a significant main group effect $(F(1,42)=6.13, p<0.017)$. Interestingly, a significant interaction effect $(F(1,42)=4.45, p<0.018)$ was observed, suggesting that the pattern of cleaved caspase- 
$3^{+}$expression differs along the sites of the lesion depending on the group (vehicle versus Neuro-Cells). Post hoc analysis revealed a significant decrease in cleaved caspase- $3^{+}$cells in rats treated with Neuro-Cells at the CA ( $p<0.003$; Figure 7E and 7F) and at the CE $(p<0.012)$, suggesting a decreased apoptosis in both segments. The number of cleaved caspase $-3^{+}$cells did not differ between Neuro-Cells and vehicle-treated rats at the RO site of the lesion (Figure 7E and 7F).
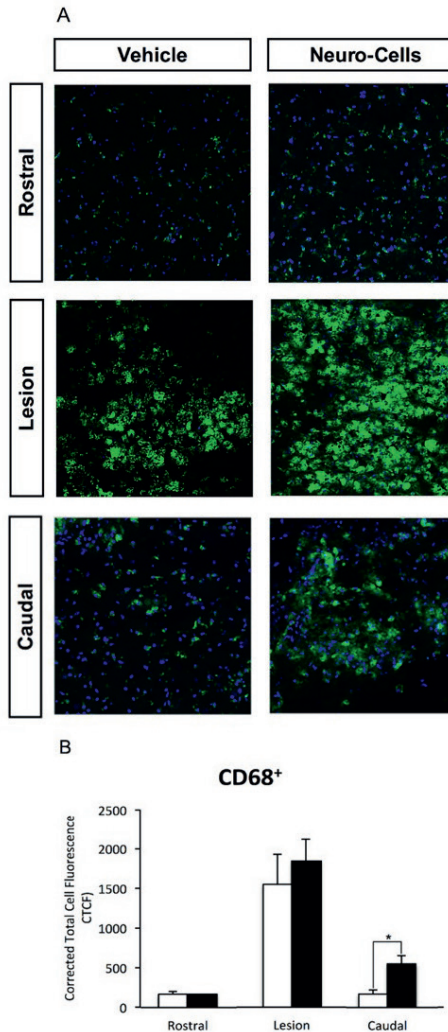

c
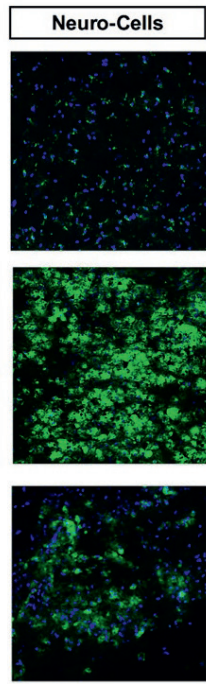

D
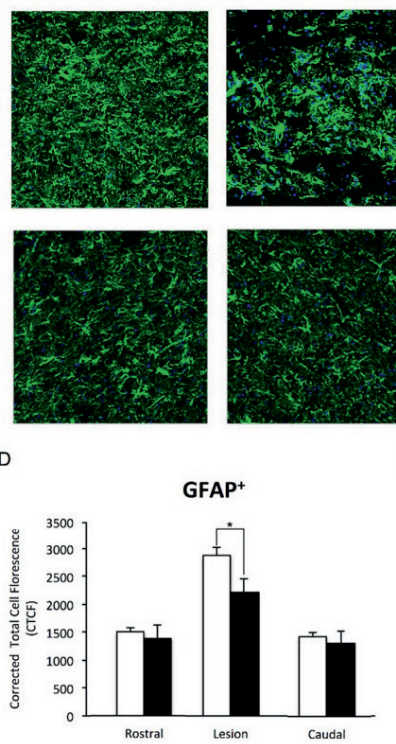

$\mathrm{E}$
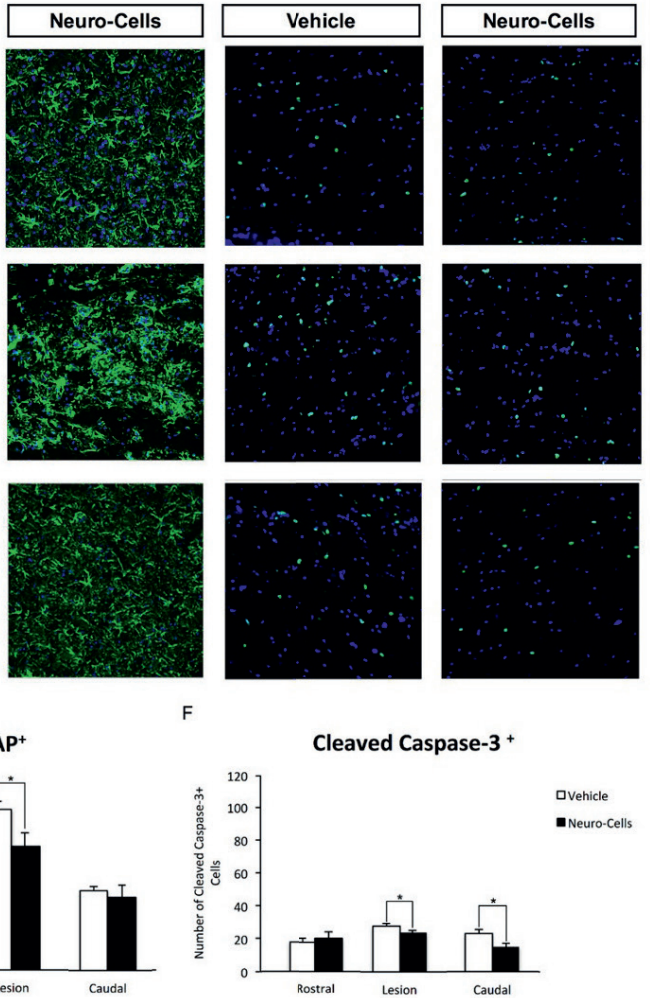

Figure 7: $\mathrm{CD}^{+} 8^{+}, \mathrm{GFAP}^{+}$and cleaved caspase- $3^{+}$cells in Veh.- and NC-treated rats across the RO, CE and $C A$ sites (Lesion $=\mathrm{CE}$ ) relative to the lesion at day 4 post-injury. Panels show representative examples of fluorescent labeling of $\mathrm{CD} 68^{+}(\mathrm{A}), \mathrm{GFAP}^{+}(\mathrm{C})$ and caspase $-3^{+}$cells $(\mathrm{E})$. For the caspase $-3^{+}$ cells we quantify the total number of positive cells $(E)$. Magnification bar represents $50 \mu \mathrm{m}$. The effects of $\mathrm{NC}$ in $\mathrm{CD}^{+} 8^{+}, \mathrm{GFAP}^{+}$and caspase $-3^{+}$at the RO, CE and CA sites are shown (B, D, F). The data are expressed as mean \pm SEM. $n=7-8$ sections per animal in each group. ${ }^{*}$ : significant, $p \leq 0.05$. 
Late effects (day 56) on cellular responses

At day 56 , we found a significant effect of the SCI-lesion site $(F(1,40)=54.72, p<0.001)$ with a significant increase in $\mathrm{CD} 68^{+}$expression at the $\mathrm{CE}$ site but no significant differences were observed between Neuro-Cells- and vehicle-treated rats at the RO, CE and CA sites (Figure 8A and $8 B)$. A significant main effect of the lesion site $(F(1,41)=6.56, p<0.003)$ with an increase in $\mathrm{GFAP}^{+}$expression was also found at the CE site (Figure $8 \mathrm{C}$ ). Post hoc analysis revealed no differences in $\mathrm{GFAP}^{+}$expression between Neuro-Cells- and vehicle-treated rats at any site of the lesion (Figure 8D). A significant main effect of the lesion site $(F(1,42)=8.67, p<0.001)$ was found in the number of cleaved caspase $-3^{+}$cells but post hoc comparisons between NeuroCells and vehicle-treated rats did not reach significance, suggesting that the number of cells that are undergoing apoptosis was not different between both groups 56 days post-injury (Figure $8 \mathrm{E}$ and $8 \mathrm{~F}$ ).

A
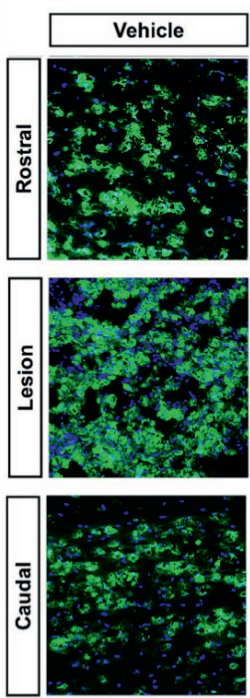

B

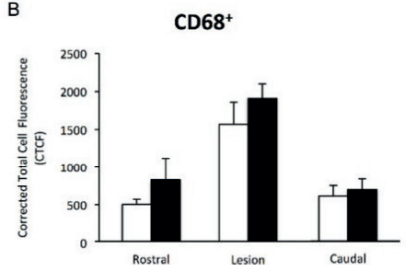

C
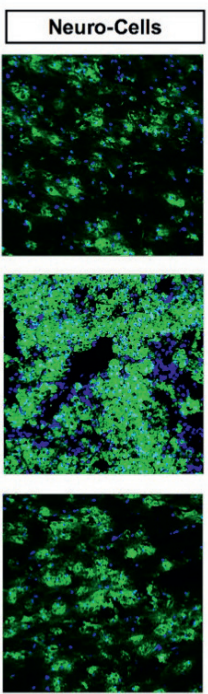

D
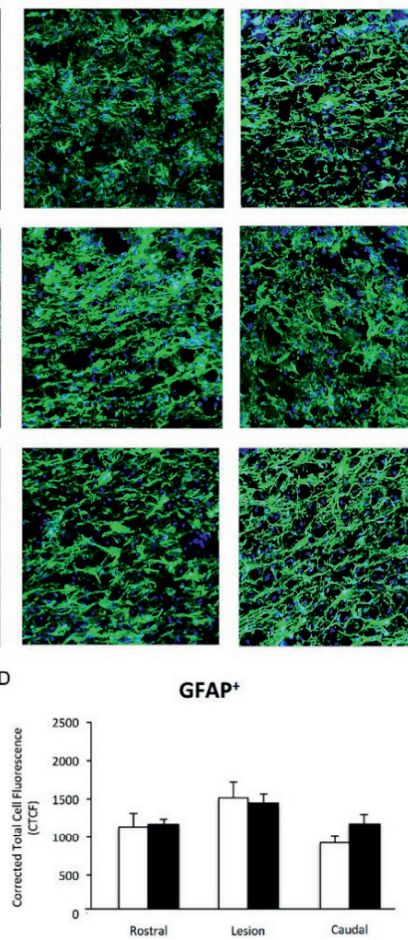

E
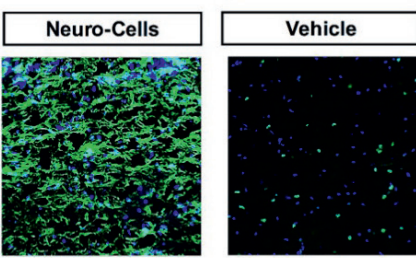

Neuro-Cells
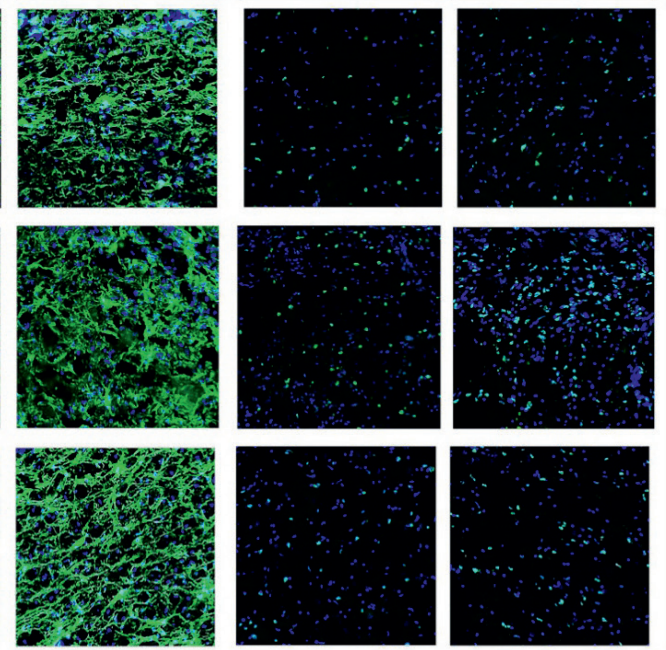

Cleaved Caspase- $3^{+}$

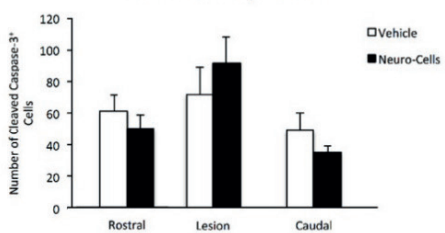

Figure 8: $\mathrm{CD}^{+} 8^{+}, \mathrm{GFAP}^{+}$and cleaved caspase- $3^{+}$cells in Veh.- and NC-treated rats across the RO, CE and $C A$ sites (Lesion $=C E$ ) relative to the lesion at day 56 post-injury. Panels show representative examples of fluorescent labeling of $\mathrm{CD} 68^{+}(\mathrm{A}), \mathrm{GFAP}^{+}(\mathrm{C})$ and caspase $-3^{+}$cells $(\mathrm{E})$. For the caspase $-3^{+}$ 
cells we quantify the total number of positive cells $(E)$. Magnification bar represents $50 \mu \mathrm{m}$. The effects of $\mathrm{NC}$ in $\mathrm{CD}^{+} 8^{+}, \mathrm{GFAP}^{+}$and caspase $-3^{+}$at the RO, CE and CA sites are shown (B, D, F). The Data are expressed as mean \pm SEM. $n=7-8$ sections per animal in each group. ${ }^{*}$ : significant, $p<0.05$.

\section{Proteomic Studies}

\section{Differential protein expression}

We quantified 573 protein abundances in $\mathrm{SCl}$ rats that were injected with Neuro-Cells and in $\mathrm{SCl}$ rats that were injected with vehicle at day 56 after administration. For this purpose, we used tissues from the three different anatomical sites of the lesion: i) rostral (RO), ii) center (CE), and iii) caudal (CA). We compared the functional analyses of the differentially abundant proteins with alternative functional classifications to assess the biological functionality relevant to the Neuro-Cells effects (see Methods). The most relevant representations appeared to be Regulons, Gene Ontology Cellular Component and Reactome pathways taken in a gene-sharing network context.

\section{Network of the functional responses: Reactome pathways}

Analysis of the sets of proteins that significantly changed their abundance in response to Neuro-Cells (with q-value below 0.05) in context of Reactome pathways, and limiting the search to pathways affected in either direction with $p<0.001$, revealed 17 responsive pathways at RO, 33 at CE and 17 at CA. The total number of pathways that responded in any direction at any of the 3 levels of the lesion was 60 . In order to reveal more general functional patterns that transcend categories of individual pathways, we constructed a protein-sharing urine network. Out of 60 responsive pathways, 55 ( 92\%) shared the underlying responsive proteins with at least one another pathway. Overall, all the 55 pathways were found in 3 densely connected clusters and 2 outlier clusters of 2-3 pathways (Figure 9). While those clusters were identified on the basis of connectivity only, irrespective to the sign of response of their members to Neuro-Cells, subsequent visualization of the directions of the effect of Neuro-Cells on expression of the 55 pathways at every level of the lesion revealed that the highly connected clusters also showed a trend to consistent sign of response at a particular level, despite the much less consistent responses at pathway (and especially - individual protein) levels between the site of the lesion. Hence, by generalizing the considered biological entity from protein to pathway and then to network of pathways, 
we found a progressively more consistent response to Neuro-Cells-treated rats at the different levels.

In particular, the first cluster from the left was enriched in glycosylation, extracellular matrix, scavenger receptors, keratan and collagen metabolism related proteins. This cluster showed a clear trend to be impaired at the RO and CE sites, and to be upregulated at the CA site, although this last trend was no longer present after multiple testing correction. The second cluster, in the middle of the network, was enriched in generally defined functional pattern of ion movements across membrane (including such categories as cardiac construction, ion homeostasis, ion transport, reduction of cytosolic $\mathrm{Ca}^{2+}$ levels, ion transport-by-type-ATPases) and semaphorins. This middle cluster had a trend to be upregulated by Neuro-Cells at all the 3 sites of the lesion. The rightmost smaller cluster of 10 pathways was not connected in as dense manner as the major 2 clusters, and the response pattern of the pathways comprising it, was more site-dependent. Interestingly, "metabolism of carbohydrates" operated as a link between the two highly connected clusters with opposite trend of response to Neuro-Cells. "Extracellular matrix organization" also provided some protein-sharing links between the two clusters. 
A

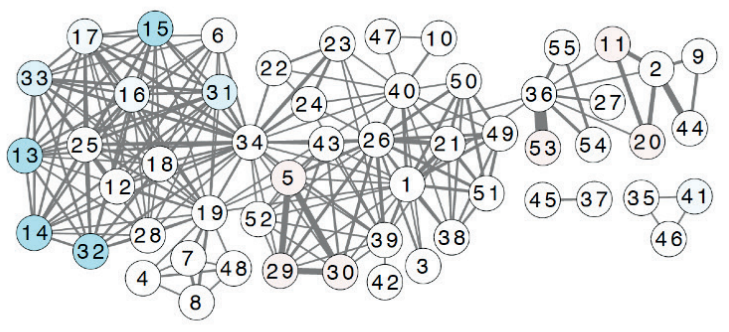

B

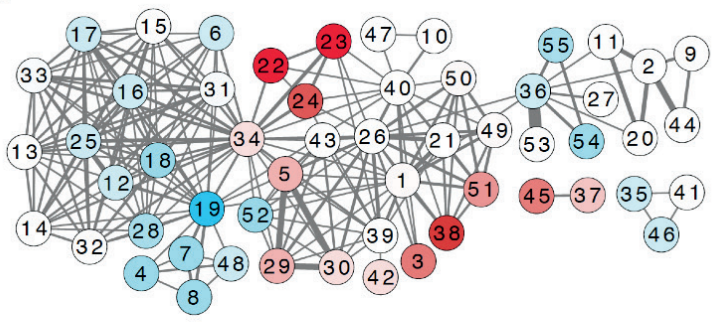

C

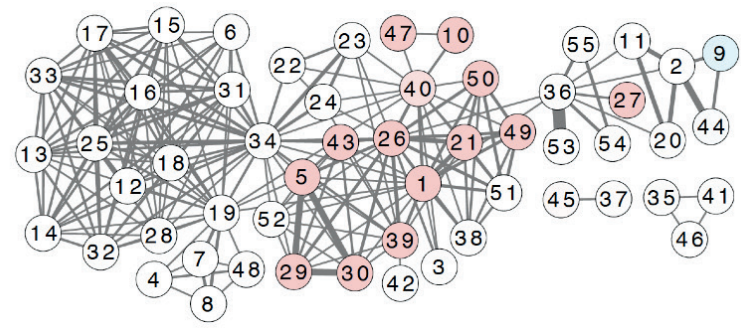

D

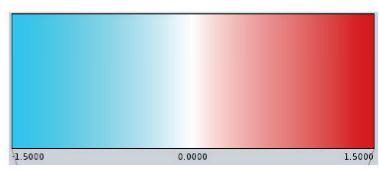

Figure 9: Protein-sharing network of the $\mathbf{5 5}$ responsive Reactome pathways that share proteins assigned to them. Significance of the pathway enrichment among proteins overexpressed with Neuro-Cells is mapped to red color, while pathway enrichment among lower expressed proteins is mapped to blue color. A, Rostral part of the lesion; B, Central part of the lesion; C, Caudal part of the lesion; D, Color map showing the range of - $\log 10(F D R)$ values. Numerical pathway IDs: 1 , Platelet activation, signaling and aggregation; 2, Respiratory electron transport, ATP synthesis by chemiosmotic coupling, and heat production by uncoupling proteins; 3, Advanced glycosylation end product receptor signaling; 4, Binding and Uptake of Ligands by Scavenger Receptors; 5, Cardiac conduction; 6, Chondroitin sulfate/dermatan sulfate metabolism; 7, Collagen biosynthesis and modifying enzymes; 8, Collagen formation; 9, Complex I biogenesis; 10, COPII (Coat Protein 2) Mediated Vesicle Transport; 11, Cristae formation; 12, CS/DS degradation; 13, Defective B4GALT1 causes B4GALT1-CDG (CDG-2d); 14, Defective CHST6 causes MCDC1; 15, Defective ST3GAL3 causes 
MCT12 and EIEE15; 16, Diseases associated with glycosaminoglycan metabolism; 17, Diseases of glycosylation; 18, ECM proteoglycans; 19, Extracellular matrix organization; 20, Formation of ATP by chemiosmotic coupling; 21, G alpha (12/13) signaling events; 22, Gluconeogenesis; 23, Glucose metabolism; 24, Glycolysis; 25, Glycosaminoglycan metabolism; 26, Hemostasis; 27, HuR (ELAVL1) binds and stabilizes mRNA; 28, Integrin cell surface interactions; 29, Ion homeostasis; 30, Ion transport by P-type ATPases; 31, Keratan sulfate/keratin metabolism; 32, Keratan sulfate biosynthesis; 33, Keratan sulfate degradation; 34, Metabolism of carbohydrates; 35, Metabolism of fat-soluble vitamins; 36, Metabolism of RNA; 37, Metallothioneins bind metals; 38, Netrin-1 signaling; 39, Neurotransmitter receptors and postsynaptic signal transmission; 40, Neutrophil degranulation; 41, RA biosynthesis pathway; 42, Recycling pathway of L1 R-HSA-437239; 43, Reduction of cytosolic Ca++ levels; 44, Respiratory electron transport; 45, Response to metal ions; 46, Retinoid metabolism and transport; 47, Retrograde transport at the Trans-Golgi-Network; 48, Scavenging by Class A Receptors; 49, Sema4D in semaphorin signaling; 50, Sema4D induced cell migration and growth-cone collapse; 51, Semaphorin interactions; 52, Smooth Muscle Contraction; 53, SRP-dependent cotranslational protein targeting to membrane; 54, Transport of Mature mRNA derived from an Intron-Containing Transcript; 55, Transport of Mature Transcript to Cytoplasm.

\section{Insights on the upstream transcriptional regulatory events from proteomics}

Besides functional summarization of the observed protein-level changes, we also leveraged the known transcription factor - target gene relationships to gain preliminary insights on the possible upstream gene regulatory events mediating the observed responses to Neuro-Cells. While transcriptome data is required to draw this level of conclusions more directly and accurately and post-transcriptional and post-translational events alter the gene expressionbased signatures. We used backward projection of the en masse protein level changes, in order to map them to known sets of transcription factor targets to identify the responsible upstream transcriptional regulators. With this approach, 33 regulons were identified to be upregulated in Neuro-Cells injected rats compared to vehicle-treated $\mathrm{SCl}$ rats at the $\mathrm{CE}, 17$ regulons at the RO and 21 regulons at the CA respectively (S3, Table 2$)$. When we examined the functions of the candidate regulons of the 3 areas, we found that the regulons identified at the CA and CE level had an identical reported function. In those regions 13 upregulated regulons involved in differentiation could be identified, though none at RO. These CA and CE situated regulons play a role in spinal cord motor neuron differentiation (LMO4), neuron differentiation (NLX2-2, RUNX2), astrocyte differentiation (NKX2-2, PAX6) and oligodendrocyte differentiation (OLIG1, OLIG2, SOX10). GOCC analysis revealed that in the actin cytoskeleton and actin filament the regulons identified at the CE were mainly located in

the plasma membrane, whereas the regulons identified at the CA and at the RO were located in the mitochondrial membrane (Table 3, S4). 


\section{Discussion}

A large body of literature provides evidence for the beneficial effects of cell-based therapies in patients with $\mathrm{SCl}$ (51). Many studies have investigated the effects of stem cells as a potential treatment for $\mathrm{SCl}$, however most of the studies failed to demonstrate safety and substantial functional improvement, after the use of manipulated stem cells (52). Manipulation of stem cells before administration leads to proliferation and/or to differentiation in a disease-free signalling environment that is not relevant to the initial aim of the transplantation. Without exposure to signalling proteins in the direct disease environment, active labelling and/or expanding will change the phenotype and function of the stem cells, and will bring these cells into the next stage of differentiation in which they are able to expand and/or to exert only basic functions. The latter, however, is key to the working mechanism in neurodegenerative disorders (30). Mesenchymal stem cells (MSCs) and Hematopoietic Stem Cells (HSCs) are not able to pass the blood-brain and central nervous system (CNS)-CSF barrier and after intrathecal application float in the CSF (53-55). However, signalling proteins are able to freely trespass these barriers and thus interact with these cells $(56,57)$. We have developed therefore a standardized bone marrow fraction of not- or marginally-manipulated MSCs and HSCs (Neuro-Cells), which can be intrathecally injected into the disease environment.

By depleting the erythrocytes and the majority of the lymphocytes of the original bone marrow-derived stem cell preparation, Neuro-Cells is suitable to be injected straight into the cerebrospinal fluid without eliciting an inflammatory reaction (rejection) $(58,59)$. The advantage of Neuro-Cells over similar products is the fact that the HSCs and MSCs are not labelled or expanded, but are kept in their original state until intrathecally administered, allowing the exposure to the disease specific signaling environment and thus activating the release of paracrine factors by the stem cells to better control the neuron hostile environment (60-63).

Both bone-marrow- and adipose-tissue-derived stem cells seem to improve motor dysfunction, though the exact mechanisms remain unclear. In this study, the human bonemarrow derived stem cells (Neuro-Cells) offer the advantage that they were not labelled nor expanded when infused directly into the cerebrospinal fluid in $\mathrm{SCl}$-treated rats, they interact 
with the disease specific signaling environment (64-67). We speculate that the beneficial effects of Neuro-Cells during the first days after its intervention in acute $\mathrm{SCl}$ might be induced by its anti-inflammatory properties, as evidenced by histological findings and differences in serum interleukin concentrations at day 4 of the study. 56 Days after this intervention, histological analysis did not reveal any signs of anti-inflammatory properties anymore, though proteomics then provided enough insights in regulons and pathways to suggest that the improvement in locomotor activity at that time was rather associated with neurogenesis and axonal regeneration. Further research, though, is needed to indeed get proof of stem cell-exerted dedicated apocrine effects (maybe through extracellular vesicles), induced by the specific pathological environment.

Several studies have shown that the therapeutic activity of MSCs is mainly mediated by MSCsecreted factors, suggesting the possible involvement of extracellular vesicles (EVs) as important players mediating the therapeutic effects of cells being used as therapeutics (such as MSCs and endothelial cells) via regenerative and anti-inflammatory modes of action (6467). For instance, MSC-EVs were found to exert immune-suppressive effects, by enforcing M2 macrophage polarization and stimulating T cell induction (68).

\section{Early-onset effects of Neuro-Cells in SCI}

Treatment with Neuro-Cells $24 \mathrm{~h}$ after surgery did not induce serious side effects or mortality in $\mathrm{SCl}$-lesioned and sham-lesioned rats. Apart from a higher incidence of a temporary Baycalresponsive cystitis in the NC-treated animals $(P<0.12)$, their 'well-being', body weight, and bladder control recovery during the entire study was comparable to that in Vehicle-treated rats (S5, Table 2, and S6, Figure 3). Importantly, not only SCl-related survival (serious adverse events leading to human end points) reached significance, NC-treated animals also reached (significant) better BBB scores.

Considering the histological analyses, rats treated with Neuro-Cells initially showed a significant reduction in $\mathrm{GFAP}^{+}$expression at $\mathrm{CE}$, indicative for a lower number of reactive astrocytes. These glial cells are, amongst others, one of the key players in scar tissue formation following an insult to the spinal cord. Previously, it has been suggested that the improved motor function is associated with a reduction in astrogliosis (69). This question, though, is beyond the scope of the current study. 
Furthermore, we showed that an early intervention with Neuro-Cells significantly increased the $\mathrm{CD} 8^{+}$cells, caudal of the lesion, 5 days after the acute injury. Several studies testing cell-based therapies in $\mathrm{SCl}$ have reported a decrease in inflammation, however it must be noted that different markers have been used to assess inflammation in $\mathrm{SCl}$ rats. In our study, we used CD68, which is frequently used as a marker for microglia and peripheral-derived macrophages, two cell types that are morphologically indistinguishable from each other. The primary role of microglia/macrophages is to remove tissue and cellular debris, thereby enabling the resolution of inflammation and tissue repair (70). Since blood-derived macrophages enter the site of injury after approximately 3 days, we speculate that our results mainly reflect the activation, and possibly the proliferation of resident microglia at the lesion and caudal site of the lesion following $\mathrm{SCl}$ and the rapid injection of viscous NeuroCells (71). The residual presence of human macrophages in Neuro-Cells may be another factor explaining the increased $\mathrm{CD}^{+} 8^{+}$cells caudal to the lesion site in the rats treated with the xenotransplant. The anti-CD68 antibody used for immunostaining was specific for rat as well as for human proteins, thus possibly reflecting an immune reaction to the xenotransplant. It has also been reported that subpopulations of HSCs and MSCs do express $\mathrm{CD} 68$, which could explain the increased expression of $\mathrm{CD}^{+} 8^{+}$we observed caudal to the lesion and the lesion itself. Over time, due to migration and differentiation, the number of the $\mathrm{CD}_{68}^{+}$cells will eventually increase at all levels.

Interestingly, we found significantly less apoptotic cells in the same anatomical regions (central and caudal part of the SCl-induced lesion) in the Neuro-Cells-treated $\mathrm{SCl}$ rats compared to the vehicle-treated rats. We found that 4 days after infusion with Neuro-Cells, rats in those parts of the lesion had significantly less caspase $-3^{+}$cells. The latter is in line with a recent study indicating that transplantation of bone-marrow stem cells in $\mathrm{SCl}$ mice downregulated the caspase $-3^{+}$cells after $\mathrm{SCl}(72)$.

Importantly, cells that stained positive for the human mitochondria antibody were detected at CA and only few at CE in NC-treated rats, 4 days after administration (Figure 6). These results may suggest that Neuro-Cells migrated from the intrathecal space to the lesion and are able to integrate into the spinal cord tissue. Since only a few positive cells were detected at the lesion, these data also may suggest that the majority of the Neuro-Cells remained in the intrathecal space. Urdzikova et al. studying the immunomodulatory effects of human- 
derived MSCs administered in immune-competent rats with $\mathrm{SCl}$ have reported similar observations (73).

\section{Late-onset effects of Neuro-Cells in SCI}

Neuro-Cells continued to improve motor recovery during the 35 days post-injury observation. No effects though were found in $\mathrm{GFAP}^{+}, \mathrm{CD}^{+} 8^{+}$cells as well as the number of cleaved caspase- $3^{+}$cells in Neuro-Cells-treated as compared to vehicle-treated T-cell deficient $\mathrm{SCl}$ rats at any site of the lesion, at 56 days after administration. We speculate that the recovery has already happened during the study period resulting in no detectable difference in GFAP and CD68 positive cells. Moreover, it appears that Neuro-Cells-treated SCI rats had a higher number of cleaved caspase $-3^{+}$cells at the lesion 57 days post-injury, which may be explained by a recently demonstrated role of caspase-3 in mediating the differentiation of HSCs $(74,75)$. Since Neuro-Cells also contains HSCs, we speculate that HSCs pass on their immunomodulatory effect to resident cells. However, it must be noted that small changes in long tracts can result in a significant improvement of locomotor functions and are not always related to the extent of the lesion (76).

The regulation of spinal cord injury is possibly a function of complex pathway interactions of cellular and biochemical reactions to trauma-induced primary and secondary inflammatory processes and a dense scar formation. Protein expression profiling in $\mathrm{SCl}$ rats injected with Neuro-Cells 56 days after treatment clearly demonstrated a distinct spatial localization at the rostral and caudal sites of the lesion, which is in agreement with literature $(77,78)$. Our clustering analysis revealed upregulated regulons at the lesion that are involved in positive regulation of differentiation (e.g. astrocyte differentiation, neuron differentiation). Our analysis took into account the spatial distribution of proteins between Neuro-Cells- and vehicle-treated rats. To a certain extent, the same pattern was observed at CA but not at RO. Among the regulons that are significantly upregulated at CA are the LMO4, which is involved in ATP signaling that promotes neuron survival after hypoxia (79), and Olig1 and Olig2, which are linked to the development and maturation of oligodendrocytes $(80,81)$. Both for CA and $\mathrm{CE}, \mathrm{NKX} 2.2$, a regulon that has a critically important role in the differentiation of adult oligodendrocyte progenitor cells (OPCs) into remyelinating oligodendrocytes was significantly upregulated (82). Moreover, upregulation of HIF1A in the lesion itself is linked to regeneration of lost or damaged tissue in mammals that have a repair response (83). 
Interestingly, in CE and CA, regulons of human origin could be identified (e.g. UBE2L3, ANXA7, ENO1, MYH11, HNRNPA0, TPD52), regulons which role is still obscure, thus providing a novel protein list through which NC might exert its recovery effects. This result may support previous evidence, suggesting that MSCs rapidly pass on their effect to resident cells, which may substantially mediate the NC-induced immunomodulatory and regenerative effects (84). SCI regulation might be the result of complex cellular and biochemical pathway interactions during primary and secondary inflammatory processes and scar formation.

In the context of reactome pathways, we found a cluster enriched in glycosylation, keratan and collagen metabolism related proteins that appeared to be downregulated at the caudal site of the lesion and at the lesion but upregulated at the rostral site of the lesion. It has been reported, that increased $\mathrm{N}$-glycosylation correlates in vivo with increased astrogliosis (85). Down regulation of glycosylation in Neuro-Cells-treated rats may be responsible for the reduced astrogliosis we observed at all sites of the lesion. Interestingly, inhibition of collagen matrix formation in spinal cord lesions have also been associated with axonal regeneration and motor function recovery (86). An upregulation in ion movements across membrane (for instance the Ion transport by P-type_ATPases) and semaphorins was identified at all three sites of the lesion. P-type ATPases is a protein family that plays an important role in the ATPdependent flipping of phospholipids across the cell membranes, a biological process that is vital in vesicle trafficking. Semaphorins are expressed in the adult nervous system and have been implicated in controlling axon guidance (87-89). However, contrary to netrins, the role of semaphorins in regeneration has not been studied extensively. At the lesion, Netrin-1 and L1CAM related proteins are found to be significantly upregulated. In a rat model of nerve injury, Ke et al. have shown that overexpression of Netrin-1 in bone-marrow-derived MSCs improves functional recovery (90). The LICAM immunoglobulin family has been involved in the promotion of regenerative axon sprouting and functional improvement after CNS injury as well as neuronal migration and synapse formation (91-93). The significant upregulation of platelet activation observed at the rostral site of the lesion has previously shown to be associated with inflammation, angiogenesis, and tissue regeneration. Platelets-derived microparticles were found to trigger endogenous stem cell repair mechanisms after middle cerebral artery occlusion in rats (94). Our proteomic analysis revealed both a signal of plasma-membrane and extracellular space restructuring processes at the lesion and 
unfolded another two ion transport gene sets at all three sites of the lesion (S7, Table 5), suggesting a paracrine effect of Neuro-Cells in $\mathrm{SCl}$ rats.

Several studies have shown that the therapeutic activity of MSCs is mainly mediated by MSCsecreted factors, suggesting the possible involvement of extracellular vesicles (EVs) as important players mediating the therapeutic effects of cells being used as therapeutics (such as MSCs and endothelial cells) via anti-inflammatory and apocrine modes of action (63-66)). For instance, MSC-EVs were found to exert immune-suppressive effects, by enforcing M2 macrophage polarization and stimulating T cell induction (67). Extracellular vesicles (EVs), which include micro-vesicles, play an important role in intercellular communication both under physiological as well as pathophysiological conditions $(95,96)$. EVs are carriers of active molecules regulating in a paracrine or endocrine manner the recipient cells $(97,98)$.

Taken together, in this particular experimental setting, Neuro-Cells did induce a beneficial effect over Vehicle in the treatment of acute balloon compression-induced immunedeficient $\mathrm{SCl}$-rats, significantly reducing $\mathrm{SCl}$-related mortality and improving locomotor recovery. The advantage of applying low-immunogenic stem cells into T-cell derived immune-deficient rats is the prevention for rejection of the graft and/or host-versus-graft disease, without the co-application of immunosuppressive drugs, which may interfere with stem cell activities $(33,34)$. Therefore, the significant acceleration of the natural, spontaneous recovery after SCl-lesioning in these rats, as shown in this research project, indeed, might be interpreted as a main effect of the stem cell transplant. The disadvantage in those immune-deficient rats, though, is that due to the reduced secondary posttraumatic inflammation in these animals, final balloon compression-induced damage with consequent sensorimotor and neurovegetative symptoms is significantly less when compared to immune-competent rats. As stem cells are supposed to reach their effects in acute lesions by suppressing (secondary) inflammation, it is thus realistic to expect a lower magnitude of their eventual beneficial effect in SCl-lesioned T-cell deficient rats. Another disadvantage is that those animals, indeed, especially after an intervention with inflammation-reducing stem cells, are more prone to infections (35). Most probably, this trend $(p<0.12)$ to a higher frequency of bladder infections in our NC-treated $\mathrm{SCl}$-lesioned rats (as compared to the placebo-treated rats) might be explained in this way. 


\section{Conclusion}

Our study provided the first proof of concept that Neuro-Cells improved the survival and accelerated natural motor recovery after $\mathrm{SCl}$. The effects of Neuro-Cells on pathological processes related to acute $\mathrm{SCl}$ are multiple. We speculate that an intrathecal injection of Neuro-Cells in spinal cord-injured rats, after some days, improved motor function initially, but not later on, in combination with a decrease of interleukins concentrations confirmed by histological established reduction of astrogliosis and apoptosis. The continued motor recovery may suggest that Neuro-Cells exert their effects on a dual role: 1) at an early stage via their anti-inflammatory properties and 2) resulting at a later stage in a modulation of the injury environment. Overall, by targeting multiple mechanistic pathways, the infused NeuroCells may induce or pass on certain aspects of their regenerative properties, providing plausible biological mechanisms involved in the regenerative process. Therefore, this treatment holds great promise as a neuroregenerative treatment including functional recovery in a clinical setting with (sub)acute $\mathrm{SCl}$ patients.

\section{Acknowledgments}

We thank Prof. Dr.G. Bos for the bone marrow procurements and R. Mohren for the mass spectral interpretation. Dr. D. Ophelders for the partial preparation of the animal study. Dr. M. Hütten for the excellent technical help. Drs R. Donders, S. Kanatsou, L.Kessels, N. Kloosterboer, and T. Wolfs for technical help and expertise.

\section{Author Contributions}

Conception and design: BK, EW, GB, and JdM. Provision of study material or volunteers: JB, L, MH, DP and SDM. Collection and/or assembly of data: LJ, and SDM. Data analysis and interpretation: BC-P, JdM, OM, TS. Manuscript writing: EW, GB, and JdM,. Final editing of manuscript: BK, EW, and TS. 


\section{Disclosure of Potential Conflicts of Interest}

Munter JP de, Munter S De and Wolters ECh are affiliated at Neuroplast BV. The other authors have no potential conflicts of interest. Neuroplast BV financed this study. The Scientific Board of UM, Maastricht, monitored the execution of the study.

\section{References}

1. DeVivo MJ (1997) Causes and costs of spinal cord injury in the United States. Spinal Cord 35: 809-813.

2. Norenberg MD, Smith J, Marcillo A (2004) The pathology of human spinal cord injury: defining the problems. J Neurotrauma 21: 429-440.

3. Schoenfeld AJ, Laughlin MD, McCriskin BJ, Bader JO, Waterman BR, Belmont PJ, Jr (2013). Spinal injuries in United States military personnel deployed to Iraq and Afghanistan: an epidemiological investigation involving 7877 combat casualties from 2005 to 2009. Spine 38: 1770-1778.

4. Strauss DJ, Devivo MJ, Paculdo DR, Shavelle RM (2006) Trends in life expectancy after spinal cord injury. Arch Phys Med Rehabil 87: 1079-1085.

5. Gupta R, Bathen ME, Smith JS, Levi AD, Bhatia NN, et al (2010) Steward O. Advances in the management of spinal cord injury. J Am Acad Orthop Surg 18: 210-222.

6. McKinley W, Meade MA, Kirshblum S, Barnard B (2004) Outcomes of early surgical management versus late or no surgical intervention after acute spinal cord injury. Arch Phys Med Rehabil 85: 1818-1825.

7. Silva NA, Sousa N, Reis RL, Salgado AJ (2014) From basics to clinical: a comprehensive review on spinal cord injury. Prog Neurobiol 114: 25-57.

8. Lawrence S (2016) Spinal Cord Injuries Treatment \& Management. Medscape, news \& perspective drugs and diseases. CME \& EDUCATION; Update May 12, 2016.

9. Alexander JK, Popovich PG (2009) Neuroinflammation in spinal cord injury: therapeutic targets for neuroprotection and regeneration. Prog Brain Res 175: 125-37.

10. Donnelly DJ, Popovich PG (2008) Inflammation and its role in neuroprotection, axonal regeneration and functional recovery after spinal cord injury. Exp Neurol 209: 378-388.

11. Blight AR (1983) Cellular morphology of chronic spinal cord injury in the cat: analysis of myelinated axons by line-sampling. Neuroscience 10: 521-543.

12. Bunge RP, Puckett WR, Becerra JL, Marcillo A, Quencer RM (1993) Observations on the pathology of human spinal cord injury. A review and classification of 22 new cases with details from a case of chronic cord compression with extensive focal demyelination. Adv Neurol 59: 75-89.

13. McDonald JW, Sadowsky C (2002) Spinal-cord injury. Lancet 359: 417-425.

14. Bracken MB (2002) Steroids for acute spinal cord injury. Cochrane Database Syst Rev 3: CD001046.

15. Botelho RV, Daniel JW, Boulosa JL, Colli BO, Farias Rde L, et al. (2009) Effectiveness of methylprednisolone in the acute phase of spinal cord injuries--a systematic review of randomized controlled trials. Rev Assoc Med Bras 55: 729-737.

16. Suberviola B, Gonzalez-Castro A, Llorca J, Ortiz-Melon F, Minambres E (2008) Early complications of high-dose methylprednisolone in acute spinal cord injury patients. Injury 39: 748-752. 
17. Antonic A, Sena ES, Lees JS, Wills TE, Skeers P, et al. (2013) Stem Cell Transplantation in Traumatic Spinal Cord Injury: A Systematic Review and Meta-Analysis of Animal Studies. PLoS Biology11: e1001738.

18. Akyurekli C, Le Y, Richardson RB, Fergusson D, Tay J, et al. (2015) A systematic review of preclinical studies on the therapeutic potential of mesenchymal stromal cell-derived microvesicles. Stem Cell Rev 11:150-160.

19. Neirinckx V, Agirman G, Coste C, Marquet A, Dion V, et al. (2015).Adult bone marrow mesenchymal and neural crest stem cells are chemoattractive and accelerate motor recovery in a mouse model of spinal cord injury. Stem Cell Res Ther 6:211 doi: 10.1186/s13287-015-0202-2.

20. Chen YB, Jia Q-Z, Li D-J, Sun JH, Xi S, et al. (2015) Spinal cord injury in rats treated using bone marrow mesenchymal stem-cell transplantation. Int J Clin Exp Med 8:9348-9354.

21. Zahra SA, Muzavir SR, Zafar H, Khan AA, Ahmad A (2012) Stem cells as treatment for spinal cord injury. theHealth 3:19-23.

22. Schwarting S, Litwak S, Hao W, Bahr M, Weise J, et al. (2008) Hematopoietic stem cells reduce postischemic inflammation and ameliorate ischemic brain injury. Stroke 39: 2867-2875.

23. Assinck P, Duncan GJ, Hilton BJ, Plemel JR, Tetzlaff W. (2017) Cell transplantation therapy for spinal cord injury. Nat Neurosci 20(5): 637-647.

24. Nejati-Koshki K, Mortazavi Y, Pilehvar-Soltanahmadi Y, Sheoran S, Zarghami N. (2017) An update on application of nanotechnology and stem cells in spinal cord injury regeneration. Biomed Pharmacother 90: 85-92

25. Jin MC, Medress ZA, Azad TD, Doulames VM, Veeravagu A. (2019) Stem cell therapies for acute spinal cord injury in humans: a review. Neurosurg Focus 46(3):E10.

26. Wragg A, Mellad JA, Beltran LE, Konoplyannikov M, San H, et al. (2008) VEGFR1/CXCR4positive progenitor cells modulate local inflammation and augment tissue perfusion by a SDF-1-dependent mechanism. J Mol Med (Berl); 86: 1221-1232.

27. de Munter JP, Wolters EC (2013) Autologous stem cells in neurology: is there a future? J Neural Transm 120: 65-73.

28. Zahra SA, Muzavir SR, Zafar H, Khan AA, Ahmad A (2012) Stem cells as treatment for spinal cord injury. theHealth 3:19-23.

29. Wagey R (2015) Mesenchymal cells. www.stemcell.com 29018.

30. Caplan Al (2017) Mesenchymal Stem Cells: Time to Change the Name! Stem Cells Transl Med; 6(6):1445-1451.

31. Diaz-Ruiz A, Vergara P, Perez-Severiano F, Segovia J, Guizar-Sahagún G, et al. (2004) Cyclosporin-A inhibits inducible nitric oxide synthase activity and expression after spinal cord injury in rats. Neurosci Lett 357: 49-52.

32. Guo J, Zeng $Y$, Liang $Y$, Wang L, Su H, et al. (2007) Cyclosporine affects the proliferation and differentiation of neural stem cells in culture. Neuroreport 18: 863-868.

33. Ibarra A, Correa D, Willms K, Merchant MT, Guizar-Sahagún G, et al. (2003) Effects of cyclosporin-A on immune response, tissue protection and motor function of rats subjected to spinal cord injury. Brain Res 979: 165-178.

34. Palladini G, Caronti B, Pozzessere G, Teichner A, Buttarelli FR, et al. (1996) Treatment with cyclosporine $A$ promotes axonal regeneration in rats submitted to transverse section of the spinal cord--II--Recovery of function. Journal fur Hirnforschung 37: 145153.

35. Potas JR, Zheng Y, Moussa C, Venn M, Gorrie CA, et al. (2006) Augmented locomotor recovery after spinal cord injury in the athymic nude rat. J Neurotrauma 23: 660-673.

36. Basso DM, Beattie MS, Bresnahan JC (1995) A sensitive and reliable locomotor rating scale for open field testing in rats. J Neurotrauma 12: 1-21. 
37. Vanicky I, Urdzikova L, Saganova K, Cizkova D, Galik (2001). A simple and reproducible model of spinal cord injury induced by epidural balloon inflation in the rat. J Neurotrauma 18: 1399-1407.

38. Dominici M, Le Blanc K, Mueller I, Slaper-Cortenbach I, Marini F, Krause D, et al. Minimal criteria for defining multipotent mesenchymal stromal cells. The International Society for Cellular Therapy position statement. Cytotherapy 2006;8:315-7.

39. Bruderer R, Bernhardt OM, Gandhi T, Miladinovic SM, Cheng LY, Messner S, et al. (2015). Extending the limits of quantitative proteome profiling with data-independent acquisition and application to acetaminophen treated 3D liver microtissues. Mol Cell Proteomics. pii: mcp.M114.044305.

40. Subramanian A, Tamayo P, Mootha VK et al. Gene set enrichment analysis: A knowledgebased approach for interpreting genome-wide expression profiles. Proc. Natl. Acad. Sci. USA 2005;102 (43): 15545-15550.

41. Bovolenta, LA, Acencio ML, Lemke N. HTRIdb: an open-access database for experimentally verified human transcriptional regulation interactions. BMC Genomics 2012;13, 405.

42. Cahan $\mathrm{P}$, Li H, Morris SA et al. CellNet: network biology applied to stem cell engineering. Cell 2014;158, 903-915.

43. Fabregat A, Sidiropoulos K, Garapati P et al. The Reactome pathway Knowledgebase. Nucleic Acids Res. 2016;44(D1):D481-7.

44. Durinck S, Spellman P, Birney E et al. Mapping identifiers for the integration of genomic datasets with the R/Bioconductor package biomaRt. Nature Protocols 2009;4:1184-1191.

45. Young MD, Wakefield MJ, Smyth GK et al. Gene ontology analysis for RNA-seq: accounting for selection bias. Genome biology 2010;11:R14.

46. Shannon P, Markiel A, Ozier O et al. Cytoscape: a software environment for integrated models of biomolecular interaction networks. Genome Res. 2003;13(11):2498-2504.

47. London A. et al.(2013). Microglia and monocyte-derived macrophages: functionally distinct populations that act in concert in CNS plasticity and repair. Front Cell Neurosci; $7: 34$.

48. Shechter R, London A, Varol C, Raposo C, Cusimano M, Yovel G, Rolls A, Mack M, Pluchino S, Martino G, Jung S, Schwartz M. (2009). Infiltrating blood-derived macrophages are vitalcells playing an anti-inflammatory role in recovery from spinal cord injury in mice. PLoS Med; 6:e1000113.

49. Soini $Y$, Kahlos K, Sormunen R, Saily M, Mantymaa P, Koistinen $P$, Paakko P, Kinnula V. (2005). Activation and relocalization of caspase 3 during the apoptotic cascade of human mesothelioma cells. Apmis; 113:426-435.

50. Springer JE, Azbill RD, Knapp PE. (1999). Activation of the caspase-3 apoptotic cascade in traumatic spinal cord injury. Nat Med; 5:943-946.

51. Tetzlaff W, Okon EB, Karimi-Abdolrezaee $\mathrm{S}$ et al. A systematic review of cellular transplantation therapies for spinal cord injury. J Neurotrauma 2011; 28:1611-1682.

52. Yoon SH, Shim YS, Park YH, Chung JK, Nam JH, Kim MO, Park HC, Park SR, Min BH, Kim EY, Choi BH, Park H, Ha Y (2007) Complete spinal cord injury treatment using autologous bone marrow cell transplantation and bone marrow stimulation with granulocyte macrophage-colony stimulating factor: Phase I/II clinical trial. Stem Cells 25:2066-2073.

53. Stamatovic, S. M., R. F. Keep and A. V. Andjelkovic (2008). "Brain endothelial cell-cell junctions: how to "open" the blood brain barrier." Current neuropharmacology 6(3): 179-192.

54. Jackson, J. S., J. P. Golding, C. Chapon, W. A. Jones and K. K. Bhakoo (2010). "Homing of stem cells to sites of inflammatory brain injury after intracerebral and intravenous administration: a longitudinal imaging study." Stem Cell Res Ther 1(2): 17.

55. Wood, M.J.,A.J. O'Loughlin and L. Samira (2011) "Exosomes and the blood-brain barrier: implications for neurological diseases. Ther Deliv 2:1095-1099. 
56. Bavisotto CC, Scalia F, Gammazza AM, Carlisi D, Bucchieri F, Macario EC de, Macario AJL, Cappello F, Campanella C. Review: Extracellular Vesicle-Mediated Cell-Cell Communication in the Nervous System: Focus on Neurological Diseases. Int. J. Mol. Sci. 2019;20:434-457.

57. Yáñez-Mó M, Siljander PRM, Andreu Z, Zavec AB, Borràs FE, Buzas El, Krisztina Buzas K, Casal E, et al. Biological properties of extracellular vesicles and their physiological functions. J Extracellular Vesicles 2015;4:1 (Article: 27066).

58. Assmus, B., T. Tonn, F. H. Seeger, C. H. Yoon, D. Leistner, J. Klotsche, V. Schachinger, E. Seifried, A. M. Zeiher and S. Dimmeler (2010). "Red blood cell contamination of the final cell product impairs the efficacy of autologous bone marrow mononuclear cell therapy." J Am Coll Cardiol 55(13): 1385-1394.

59. Lossinsky, A. S. and R. R. Shivers (2004). "Structural pathways for macromolecular and cellular transport across the blood-brain barrier during inflammatory conditions. Review." Histol Histopathol 19(2): 535-564.

60. Kinnaird, T., E. Stabile, M. S. Burnett, M. Shou, C. W. Lee, S. Barr, S. Fuchs and S. E. Epstein (2004). "Local delivery of marrow-derived stromal cells augments collateral perfusion through paracrine mechanisms." Circulation 109(12): 1543-1549.

61. Gnecchi, M., Z. Zhang, A. Ni and V. J. Dzau (2008). "Paracrine mechanisms in adult stem cell signaling and therapy." Circ Res 103(11): 1204-1219.

62. Hsiao, S. T., A. Asgari, Z. Lokmic, R. Sinclair, G. J. Dusting, S. Y. Lim and R. J. Dilley (2012). "Comparative analysis of paracrine factor expression in human adult mesenchymal stem cells derived from bone marrow, adipose, and dermal tissue." Stem Cells Dev 21(12): 2189-2203.

63. Dostert G, Mesure B, Menu P, Velot É (2017). How Do Mesenchymal Stem Cells Influence or Are Influenced by Microenvironment through Extracellular Vesicles Communication? Front Cell Dev Biol: 5:6.

64. Rani, S., A. E. Ryan, M. D. Griffin and T. Ritter (2015). "Mesenchymal Stem Cell-derived Extracellular Vesicles: Toward Cell-free Therapeutic Applications." Mol Ther 23(5): 812823.

65. Zhang, B., R. W. Yeo, K. H. Tan and S. K. Lim (2016). "Focus on Extracellular Vesicles: Therapeutic Potential of Stem Cell-Derived Extracellular Vesicles." Int J Mol Sci 17(2): 174

66. Harting, M. T., A. K. Srivastava, S. Zhaorigetu, H. Bair, K. S. Prabhakara, N. E. Toledano Furman, J. V. Vykoukal, K. A. Ruppert, C. S. Cox, Jr. and S. D. Olson (2018)."InflammationStimulated Mesenchymal Stromal Cell-Derived Extracellular Vesicles Attenuate Inflammation." Stem Cells 36(1): 79-90.

67. Ruppert, K. A., T. T. Nguyen, K. S. Prabhakara, N. E. Toledano Furman, A. K. Srivastava, M. T. Harting, C. S. Cox, Jr. and S. D. Olson (2018). "Human Mesenchymal Stromal CellDerived Extracellular Vesicles Modify Microglial Response and Improve Clinical Outcomes in Experimental Spinal Cord Injury." Sci Rep 8(1): 480.

68. Zhang Y, Li L, Yu J, Zhu D, Zhang Y, Li X, et al. Microvesicle-mediated delivery of transforming growth factor beta1 siRNA for the suppression of tumor growth in mice. Biomaterials. 2014;35:4390-400.

69. Ren H, Han M, Zhou J et al. Repair of spinal cord injury by inhibition of astrocyte growth and inflammatory factor synthesis through local delivery of flavopiridol in PLGA nanoparticles. Biomaterials 2014;35:6585-6594.

70. London A. et al. Microglia and monocyte-derived macrophages: functionally distinct populations that act in concert in CNS plasticity and repair. Front Cell Neurosci. 2013; 7 :34.

71. Popovich P.G. and Hickey W.F. Bone marrow chimeric rats reveal the unique distribution of resident and recruited macrophages in the contused rat spinal cord. J. Neuropathol. Ex. Neurol. 2001.

72. Noushin Gashmardi, Seyed Ebrahim Hosseini, Davood Mehrabani, Mohammad Amin 
Edalatmanesh, Zahra Khodabandeh. Impacts of Bone Marrow Stem Cells on Caspase-3 Levels after Spinal Cord Injury in Mice. Iran J Med Sci. 2017;42(6): 593-598.

73. Urdzikova, L. et al. Human mesenchymal stem cells modulate inflammatory cytokines after spinal cord injury in rat. Int. J. Mol. Sci. 2014;15:11275 - 11293.

74. Janzen V, Fleming HE, Riedt T, Karlsson G, Riese MJ, Lo Celso C, Reynolds G, Milne CD, Paige CJ, Karlsson S, Woo M, Scadden DT. Hematopoietic stem cell responsiveness to exogenous signals is limited by caspase-3. Cell Stem Cell. 2008; 2(6):584-94.

75. Fujita J, Crane AM, Souza MK, Dejosez M, Kyba M, Flavell RA, Thomson JA, Zwaka TP. Caspase activity mediates the differentiation of embryonic stem cells. Cell Stem Cell. 2008; 2(6):595-601.

76. Blight AR. Cellular morphology of chronic spinal cord injury in the cat: analysis of myelinated axons by line-sampling. Neuroscience. 1983; 10(2):521-43.

77. Devaux S, Cizkova D, Quanico J, Franck J, Nataf S, Pays L, Hauberg-Lotte L, Maass P, Kobarg JH, Kobeissy F, Mériaux C, Wisztorski M, Slovinska L, Blasko J, Cigankova V, Fournier I, Salzet M. Proteomic Analysis of the Spatio-temporal Based Molecular Kinetics of Acute Spinal Cord Injury Identifies a Time- and Segment-specific Window for Effective Tissue Repair. Mol Cell Proteomics. 2016; 15(8):2641-70.

78. Cizkova D, Le Marrec-Croq F, Franck J, Slovinska L, Grulova I, Devaux S, Lefebvre C, Fournier I, Salzet M. Alterations of protein composition along the rostro-caudal axis after spinal cord injury: proteomic, in vitro and in vivo analyses.Front Cell Neurosci. 2014; 17;8:105.

79. Chen HH, Schock SC, Xu J, Safarpour F, Thompson CS, Stewart AF. Extracellular ATPdependent upregulation of the transcription cofactor LMO4 promotes neuron survival from hypoxia. Exp Cell Res. 2007; 313(14):3106-16.

80. Cheng $X$, Wang $Y$, He Q, Qiu M, Whittemore SR, Cao Q. Bone morphogenetic protein signaling and olig1/2 interact to regulate the differentiation and maturation of adult oligodendrocyte precursor cells. Stem Cells. 2007; 25(12):3204-14.

81. Lu QR1, Sun T, Zhu Z, Ma N, Garcia M, Stiles CD, Rowitch DH. Common developmental requirement for Olig function indicates a motor neuron/oligodendrocyte connection. Cell. 2002; 109(1):75-86.

82. Fancy SP1, Zhao C, Franklin RJ. Increased expression of Nkx2.2 and Olig2 identifies reactive oligodendrocyte progenitor cells responding to demyelination in the adult CNS. Mol Cell Neurosci. 2004; 27(3):247-54.

83. Zhang Y, Strehin I, Bedelbaeva K, Gourevitch D, Clark L, Leferovich J, Messersmith PB, Heber-Katz E. Drug-induced regeneration in adult mice. Science Translational Medicine 2015; 7 (290): 290ra92.

84. Eggenhofer E, Luk F, Dahlke MH, Hoogduijn MJ. The life and fate of mesenchymal stem cells. Front Immunol. 2014; 5:148 eCollection.

85. Nourse JL, Prieto JL, Dickson AR, Lu J, Pathak MM et al. Membrane biophysics define neuron and astrocyte progenitors in the neural lineage. Stem Cells. 2014; 32(3):706-16.

86. Klapka N, Müller HW. Collagen matrix in spinal cord injury. J Neurotrauma. 2006; 23(34):422-35.

87. Pasterkamp RJ, Giger RJ. Semaphorin function in neural plasticity and disease. Curr Opin Neurobiol. 2009; 19:263-274.

88. Tran TS, Kolodkin AL, Bharadwaj R. Semaphorin regulation of cellular morphology. Annual review of cell and developmental biology. 2007; 23:263-292.

89. Yoshida Y. Semaphorin signaling in vertebrate neural circuit assembly. Frontiers in molecular neuroscience. 2012; 5:71.

90. Ke X, Li Q, Xu L, Zhang Y, Li D, Ma J, Mao X. Netrin-1 overexpression in bone marrow mesenchymal stem cells promotes functional recovery in a rat model of peripheral nerve injury. Biomed Res. 2015; 29(5):380-9.

91. Barry J, Gu Y, Gu C. Polarized targeting of L1-CAM regulates axonal and dendritic bundling 
in vitro. Eur J Neurosci. 2010; 32:1618-163.

92. Li YL, Wu GZ, Dawe GS, Zeng L, Cui SS, Loers G, Tilling T, Sun L, Schachner M, Xiao ZC. Cell surface sialylation and fucosylation are regulated by $\mathrm{L} 1$ via phospholipase Cgamma and cooperate to modulate neurite outgrowth, cell survival and migration. PLoS ONE. 2008; 3:e384.

93. Maness PF, Schachner $M$. Neural recognition molecules of the immunoglobulin superfamily: signaling transducers of axon guidance and neuronal migration. Nat Neurosci. 2007; 10:19-26.

94. Shan LY, Li JZ, Zu LY, Niu CG, Ferro A, Zhang YD, Zheng LM, Ji Y. Platelet-derived microparticles are implicated in remote ischemia conditioning in a rat model of cerebral infarction. CNS Neurosci Ther. 2013;19:917-925.

95. Raposo G, Stoorvogel W. Extracellular vesicles: exosomes, microvesicles, and friends. J Cell Biol. 2013; 200:373-383.

96. Dignat-George F, Boulanger CM. The many faces of endothelial microparticles. Arterioscler Thromb Vasc Biol. 2011; 31:27-33.

97. Yáñez-Mó M, Siljander PR, Andreu Z, et al. Biological properties of extracellular vesicles and their physiological functions. J Extracell Vesicles. 2015; 4:27066.

98. Théry C. Exosomes: secreted vesicles and intercellular communications. F1000 Biol Rep. 2011; 3:15. 


\section{Supplementary Material S1}

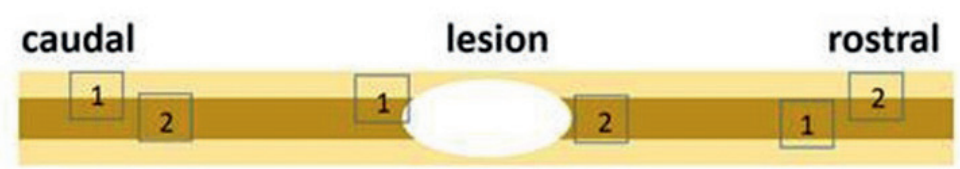

\section{Grey matter}

Figure 1: An example of a longitudinal section of the spinal cord, in which the fluorescence density was measured. Fluorescence intensity was measured using ImageJ at 2 points (the rectangles): 2 at the outer surface of the spinal cord and at the middle part of the section at the caudal and rostral part of the lesion and 2 points at the border of the lesion. 
S2.

Table 1. DIA windows

\begin{tabular}{|c|c|c|c|c|}
\hline window & start_mz & end_mz & center & width \\
\hline 1 & 350 & 366 & 358 & 16 \\
\hline 2 & 365 & 380 & 372,5 & 15 \\
\hline 3 & 379 & 392 & 385,5 & 13 \\
\hline 4 & 391 & 403 & 397 & 12 \\
\hline 5 & 402 & 414 & 408 & 12 \\
\hline 6 & 413 & 423 & 418 & 10 \\
\hline 7 & 422 & 432 & 427 & 10 \\
\hline 8 & 431 & 440 & 435,5 & 9 \\
\hline 9 & 439 & 449 & 444 & 10 \\
\hline 10 & 448 & 458 & 453 & 10 \\
\hline 11 & 457 & 466 & 461,5 & 9 \\
\hline 12 & 465 & 473 & 469 & 8 \\
\hline 13 & 472 & 481 & 476,5 & 9 \\
\hline 14 & 480 & 490 & 485 & 10 \\
\hline 15 & 489 & 497 & 493 & 8 \\
\hline 16 & 496 & 506 & 501 & 10 \\
\hline 17 & 505 & 514 & 509,5 & 9 \\
\hline 18 & 513 & 522 & 517,5 & 9 \\
\hline 19 & 521 & 530 & 525,5 & 9 \\
\hline 20 & 529 & 539 & 534 & 10 \\
\hline 21 & 538 & 547 & 542,5 & 9 \\
\hline 22 & 546 & 554 & 550 & 8 \\
\hline 23 & 553 & 562 & 557,5 & 9 \\
\hline 24 & 561 & 571 & 566 & 10 \\
\hline 25 & 570 & 579 & 574,5 & 9 \\
\hline 26 & 578 & 588 & 583 & 10 \\
\hline 27 & 587 & 596 & 591,5 & 9 \\
\hline 28 & 595 & 606 & 600,5 & 11 \\
\hline 29 & 605 & 614 & 609,5 & 9 \\
\hline 30 & 613 & 623 & 618 & 10 \\
\hline 31 & 622 & 632 & 627 & 10 \\
\hline 32 & 631 & 642 & 636,5 & 11 \\
\hline 33 & 641 & 651 & 646 & 10 \\
\hline 34 & 650 & 661 & 655,5 & 11 \\
\hline 35 & 660 & 671 & 665,5 & 11 \\
\hline 36 & 670 & 681 & 675,5 & 11 \\
\hline 37 & 680 & 691 & 685,5 & 11 \\
\hline 38 & 690 & 701 & 695,5 & 11 \\
\hline 39 & 700 & 713 & 706,5 & 13 \\
\hline 40 & 712 & 724 & 718 & 12 \\
\hline 41 & 723 & 737 & 730 & 14 \\
\hline 42 & 736 & 750 & 743 & 14 \\
\hline 43 & 749 & 764 & 756,5 & 15 \\
\hline 44 & 763 & 779 & 771 & 16 \\
\hline 45 & 778 & 793 & 785,5 & 15 \\
\hline 46 & 792 & 809 & 800,5 & 17 \\
\hline 47 & 808 & 825 & 816,5 & 17 \\
\hline 48 & 824 & 842 & 833 & 18 \\
\hline 49 & 841 & 861 & 851 & 20 \\
\hline 50 & 860 & 882 & 871 & 22 \\
\hline 51 & 881 & 906 & 893,5 & 25 \\
\hline 52 & 905 & 932 & 918,5 & 27 \\
\hline 53 & 931 & 961 & 946 & 30 \\
\hline 54 & 960 & 995 & 977,5 & 35 \\
\hline 55 & 994 & 1039 & 1016,5 & 45 \\
\hline 56 & 1038 & 1089 & 1063,5 & 51 \\
\hline 57 & 1088 & 1166 & 1127 & 78 \\
\hline 58 & 1165 & 1650 & 1407,5 & 485 \\
\hline
\end{tabular}




\begin{tabular}{|c|c|c|}
\hline Level of the Lesion & Regulon & P-value \\
\hline \multirow{20}{*}{ Caudal part of the Lesion } & TWIST1 & 0.005 \\
\hline & SOX2 & 0.005 \\
\hline & POU3F2 & 0.008 \\
\hline & HOXD10 & 0.014 \\
\hline & OLIG1 & 0.016 \\
\hline & $N K X 2-2$ & 0.019 \\
\hline & OSR2 & 0.020 \\
\hline & PAX6 & 0.021 \\
\hline & LMO4 & 0.024 \\
\hline & ARHGAP35 & 0.026 \\
\hline & RORB & 0.027 \\
\hline & ZNF536 & 0.029 \\
\hline & CHD5 & 0.029 \\
\hline & RFX4 & 0.031 \\
\hline & TBX3 & 0.032 \\
\hline & OLIG2 & 0.035 \\
\hline & FOXG1 & 0.036 \\
\hline & SRA1 & 0.039 \\
\hline & $\mathrm{ZHX3}$ & $0 ., 040$ \\
\hline & 50X8 & 0.043 \\
\hline \multirow{32}{*}{ Central part of the Lesion } & TWIST1 & 0.005 \\
\hline & ARNT2 & 0.004 \\
\hline & LMO4 & 0.006 \\
\hline & JUN & 0.007 \\
\hline & ZNF536 & 0.009 \\
\hline & PAX6 & 0.010 \\
\hline & RUNX2 & 0.012 \\
\hline & OLIG1 & 0.013 \\
\hline & THRA & 0.013 \\
\hline & SOX10 & 0.017 \\
\hline & NKX2-2 & 0.017 \\
\hline & HIF1A & 0.019 \\
\hline & ANKRD1 & 0.019 \\
\hline & ST18 & 0.021 \\
\hline & LHX2 & 0.022 \\
\hline & FOXG1 & 0.023 \\
\hline & INSM2 & 0.026 \\
\hline & RORB & 0.027 \\
\hline & CSRNP2 & 0.028 \\
\hline & SPZ1 & 0.033 \\
\hline & NPAS3 & 0.033 \\
\hline & NFATC3 & 0.037 \\
\hline & POU3F2 & 0.037 \\
\hline & ATF1 & 0.039 \\
\hline & FEZF2 & 0.040 \\
\hline & INSM1 & 0.041 \\
\hline & NPAS2 & 0.041 \\
\hline & 50X8 & 0.043 \\
\hline & NR3C2 & 0.044 \\
\hline & TBR1 & 0.044 \\
\hline & FOXQ1 & 0.045 \\
\hline & ZNF529 & 0.045 \\
\hline
\end{tabular}




\begin{tabular}{|c|c|c|}
\hline & E2F6 & 0.045 \\
\hline Rostral part of the Lesion & NAT14 & 0.045 \\
\hline & SCMH1 & 0.004 \\
AR & 0.004 \\
KLF4 & 0.008 \\
DMRT2 & 0.012 \\
& RBMX & 0.016 \\
& TBX5 & 0.020 \\
& KLF13 & 0.021 \\
& EBF2 & 0.030 \\
& FOXP3 & 0.032 \\
& TFCP2L1 & 0.033 \\
& CREB312 & 0.034 \\
& CSDE1 & 0.035 \\
& POU2F2 & 0.042 \\
& MEOX1 & 0.042 \\
& ZNF238 & 0.043 \\
& FOXC2 & 0.044 \\
& RNF10 & 0.044 \\
\hline
\end{tabular}

Table 2: Upregulated regulons at the three levels of the lesion (caudal, lesion, rostral) in NC-treated SCI rats compared to vehicle-injected SCI rats. 
S4

\begin{tabular}{|c|c|c|}
\hline Lesion Site & Cellular Compartment & P-value \\
\hline Caudal part & $\begin{array}{l}\text { Plasma_Membrane } \\
\text { Endoplasmic Reticulum Membrane } \\
\text { Membrane } \\
\text { Integral To Membrane } \\
\text { Intrinsic_To_Membrane } \\
\text { Ruffle } \\
\text { Integral To Endoplasmic Reticulum Membrane } \\
\text { Intrinsic To Endoplasmic Reticulum Membrane } \\
\text { Integral_To_Plasma_Membrane } \\
\text { Growth Cone } \\
\text { Site_Of_Polarized_Growth }\end{array}$ & $\begin{array}{l}0.024 \\
0.031 \\
0.035 \\
0.036 \\
0.036 \\
0.039 \\
0.044 \\
0.044 \\
0.044 \\
0.045 \\
0.045\end{array}$ \\
\hline Central part & $\begin{array}{l}\text { Plasma Membrane } \\
\text { Endoplasmic Reticulum Membrane } \\
\text { Membrane } \\
\text { Integral To Membrane } \\
\text { Intrinsic To Membrane } \\
\text { Ruffle } \\
\text { Integral To Endoplasmic Reticulum Membrane } \\
\text { Intrinsic To Endoplasmic Reticulum Membrane } \\
\text { Integral To Plasma Membrane } \\
\text { Intrinsic To Plasma Membrane } \\
\text { Growth Cone }\end{array}$ & $\begin{array}{l}0.024 \\
0.031 \\
0.035 \\
0.036 \\
0.036 \\
0.039 \\
0.044 \\
0.044 \\
0.044 \\
0.044 \\
0.045\end{array}$ \\
\hline Rostral part & $\begin{array}{l}\text { Site Of Polarized Growth } \\
\text { Heterogeneous Nuclear ribonucleoprotein Complex } \\
\text { Cytoskeletal Part } \\
\text { Actin Cytoskeleton } \\
\text { Plasma Membrane } \\
\text { Sarcomere } \\
\text { Actin Filament } \\
\text { Cell Projection }\end{array}$ & $\begin{array}{l}0.009 \\
0.009 \\
0.015 \\
0.030 \\
0.044 \\
0.045 \\
0, .047 \\
0.048\end{array}$ \\
\hline
\end{tabular}

Table 3: Identified upregulated GO cellular compartment (GOCC) at the lesion, caudally and rostrally to the lesion in Neuro-Cells-treated $\mathrm{SCl}$ rats. 
S5

\begin{tabular}{|l|l|l|l|}
\hline Day post-surgery & Experimental group & Mean [g] & SEM \\
\hline day1 & SCI Vehicle & 271 & 4.85 \\
\hline & SCI Neuro-Cells & 274 & 3.87 \\
\hline day35 & SCI Vehicle & 279 & 9.00 \\
\hline & SCI Neuro-Cells & 272 & 6.72 \\
\hline
\end{tabular}

Table 4: Absolute body weight recorded at day1 and day 35 post-injury. Animals that underwent spinal cord injury and treated with Neuro-Cells did not differ in body weight compared to vehicletreated group at day 1 and day 35 post-surgery.

S6

A

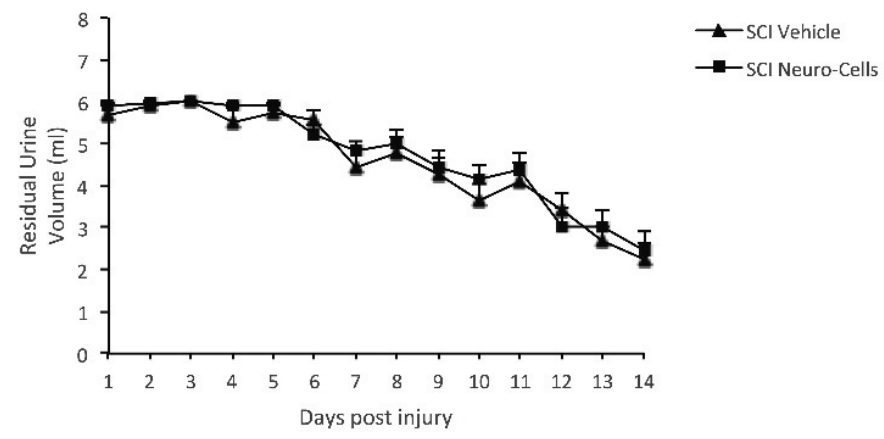

Figure 3: Bladder function. After $\mathrm{SCl}$ surgery, all animals needed assistance with bladder voiding. Treatment with Neuro-Cells did not affect the recovery of bladder control as seen in SCI-lesioned rats treated with vehicle throughout a 14 day observation period (repeated-measurement ANOVA, $p=$ 0.916; Figure 5B). 


\begin{tabular}{|c|c|}
\hline Reactome Pathway & P-Value \\
\hline Gluconeogenesis___R-HSA-70263 & $3.86 \mathrm{E}-06$ \\
\hline Glucose_Metabolism_R-HSA-70326 & $3.4 \mathrm{E}-05$ \\
\hline Netrin-1_Signaling___ R-HSA-373752 & 0.001 \\
\hline Glycolysis__R-HSA-70171 & 0.001 \\
\hline Post-Chaperonin_Tubulin_Folding_Pathway_R-HSA-389977 & 0.001 \\
\hline Advanced_Glycosylation_Endproduct_Receptor_Signaling__R-HSA-879415 & 0.001 \\
\hline Response_To_Metal_Ions__R-HSA-5660526 & 0.001 \\
\hline Semaphorin_Interactions___ R-HSA-373755 & 0.002 \\
\hline Cardiac_Conduction__ R-HSA-5576891 & 0.004 \\
\hline Ion_Homeostasis___ R-HSA-5578775 & 0.004 \\
\hline RAF-Independent_MAPK1/3_Activation_R_HSA-112409 & 0.004 \\
\hline Nuclear_Signaling_By_ERBB4__R-HSA-1251985 & 0.006 \\
\hline Metallothioneins_Bind_Metals__R-HSA-5661231 & 0.006 \\
\hline Metabolism_Of_Carbohydrates___R-HSA-71387 & 0.009 \\
\hline Recycling_Pathway_Of_L1___R-HSA-437239 & 0.009 \\
\hline Crmps_In_Sema3A_Signaling__R-HSA-399956 & 0.010 \\
\hline Signal_Transduction_By_L1___R-HSA-445144 & 0.011 \\
\hline Carboxyterminal_Post-Translational_Modifications_Of_Tubulin___R-HSA-89553332 & 0.012 \\
\hline Ion_Transport_By_P-Type_Atpases__R-HSA-936837 & 0.012 \\
\hline L1CAM_Interactions_R-HSA-373760 & 0.013 \\
\hline Ion_Channel_Transport___R-HSA-983712 & 0.018 \\
\hline Activated_TLR4_Signalling___R-HSA-166054 & 0.020 \\
\hline Myd88-Independent_TLR3/TLR4_Cascade__R-HSA-166166 & 0.020 \\
\hline Toll_Like_Receptor_3_(T|r3)_Cascade___ R-Hsa-168164 & 0.020 \\
\hline Toll_Like_Receptor_4_(TIr4)_Cascade___R-Hsa-166016 & 0.020 \\
\hline TRIF-Mediated_TLR3/TLR4_Signaling___ R-HSA-937061 & 0.020 \\
\hline Dex/Hbox_Helicases_Activate_Type_I_IFN_And_Inflammatory_Cytokines_Production__R-HSA-3134963 & 0.030 \\
\hline RIP-Mediated_Nfkb_Activation_Via_ZBP1___R-HSA-1810476 & 0.030 \\
\hline TAK1_Activates_Nfkb_By_Phosphorylation_And_Activation_Of_lkks_Complex__R-HSA-445989 & 0.030 \\
\hline ZBP1(DAI)_Mediated_Induction_Of_Type_I_Ifns___R-HSA-1606322 & 0.030 \\
\hline DSCAM_Interactions___ R-HSA-376172 & 0.031 \\
\hline MAPK1_(ERK2)_Activation__R-HSA-112411 & 0.031 \\
\hline Activation_Of_The_AP-1_Family_Of_Transcription_Factors___R-HSA-450341 & 0.032 \\
\hline Negative_Feedback_Regulation_Of_MAPK_Pathway__ R-HSA-5674499 & 0.032 \\
\hline Acyl_Chain_Remodeling_Of_CL_R-HSA-1482798 & 0.032 \\
\hline Beta_Oxidation_Of_Palmitoyl-Coa_To_Myristoyl-Coa___R-HSA-77305 & 0.032 \\
\hline PRC2_Methylates_Histones_And_DNA__R-HSA-212300 & 0.033 \\
\hline Myd88:Mal_Cascade_Initiated_On_Plasma_Membrane__R-HSA-166058 & 0.038 \\
\hline Myd88_Cascade_Initiated_On_Plasma_Membrane__R-HSA-975871 & 0.038 \\
\hline Myd88_Dependent_Cascade_Initiated_On_Endosome___R-HSA-975155 & 0.038 \\
\hline Toll_Like_Receptor_10_(T|r10)_Cascade___R-Hsa-168142 & 0.038 \\
\hline Toll_Like_Receptor_2_(TIr2)_Cascade___R-Hsa-181438 & 0.038 \\
\hline
\end{tabular}




\begin{tabular}{|c|c|}
\hline Toll_Like_Receptor_5_(TIr5)_Cascade___R-Hsa-168176 & 0.038 \\
\hline Toll_Like_Receptor_7/8_(T|r7/8)_Cascade___R-Hsa-168181 & 0.038 \\
\hline Toll_Like_Receptor_9_(TIr9)_Cascade___R-Hsa-168138 & 0.038 \\
\hline Toll_Like_Receptor_TIr1:TIr2_Cascade___R-Hsa-168179 & 0.038 \\
\hline Toll_Like_Receptor_TIr6:TIr2_Cascade___ R-Hsa-168188 & 0.038 \\
\hline TRAF6_Mediated_Induction_Of_Nfkb_And_MAP_Kinases_Upon_TLR7/8_Or_9_Activation___R-HSA-975138 & 0.038 \\
\hline TRAF6_Mediated_Induction_Of_Proinflammatory_Cytokines___R-HSA-168180 & 0.038 \\
\hline Mitochondrial_Fatty_Acid_Beta-Oxidation___R-Hsa-77289 & 0.039 \\
\hline Pyruvate_Metabolism___R-HSA-70268 & 0.040 \\
\hline RHO_Gtpases_Activate_KTN1___R-HSA-5625970 & 0.043 \\
\hline Senescence-Associated_Secretory_Phenotype_(Sasp)___ R-Hsa-2559582 & 0.043 \\
\hline Cargo_Concentration_In_The_ER___R-HSA-5694530 & 0.044 \\
\hline Oxidative_Stress_Induced_Senescence___R-Hsa-2559580 & 0.044 \\
\hline Mitochondrial_Calcium_lon_Transport____R-HSA-8949215 & 0.044 \\
\hline Toll-Like_Receptors_Cascades___R-Hsa-168898 & 0.047 \\
\hline Formation_Of_Tubulin_Folding_Intermediates_By_CCT/Tric__R-HSA-389960 & 0.049 \\
\hline
\end{tabular}

Table 5: Reactome pathways identified in Neuro-Cells-treated SCl rats at the lesion. 


\section{CHAPTER 5}

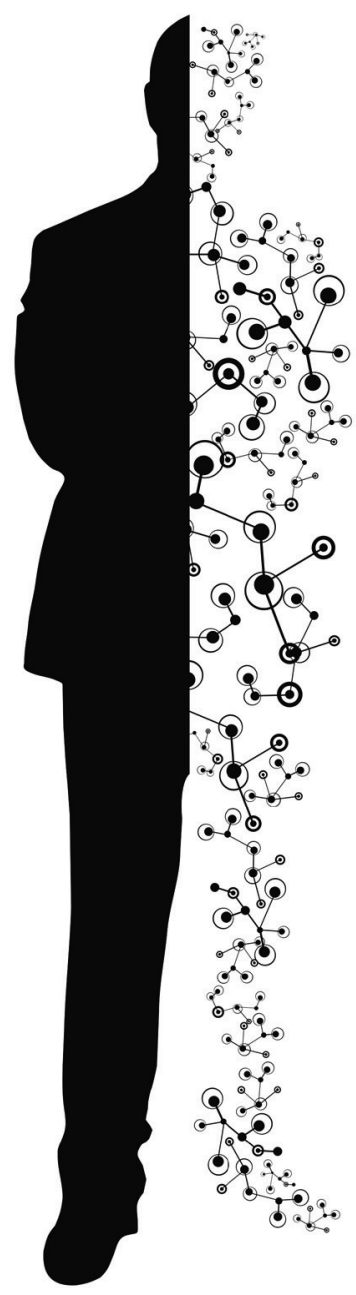




\section{Efficacy of different doses of human adult bone marrow stem cell transplantation on angiogenesis in a rat model with hind limb ischemia}

Jip Beugels, Johannes P J M De Munter, RVan der Hulst, Boris W Kramer and Eric C H Wolters 


\section{Abstract}

\section{Background}

Stem cell transplantation has been implied to facilitate angiogenesis by direct paracrine effects on signaling pathways. There are many different sources, types, dosages and routes of administration of stem cells under investigation. In this study we tested the concomitant intramuscular and intravenous administration of human mesenchymal and hematopoietic stem cells from bone marrow aspirates in three different dosages in order to establish an optimal dose for angiogenesis. For this purpose a nude $\mathrm{T}$ cell deficient rat model with hind limb ischemia was used as a model for angiogenesis which did not warrant immunosuppressive drugs.

\section{Methods and Results}

Seven days after a surgical occlusion of the A. iliaca externa in the right hind limb, a baseline digital subtraction angiography (DSA) was performed, and animals were intra-arterially and intramuscularly injected with low, medium or high concentrations of human mesenchymal stem cells or with a saline $0.9 \%$ solution (vehicle). At day 35 , DSA was repeated, and the images of both registrations were compared between the different concentration groups and the vehicle group.

DSA clearly demonstrated the presence of occlusions, resulting in moderate to severe ischemia 7 days after ligation. Comparing Mean Gray Values at day 35 DSA versus day 7 DSA, the difference did show a natural decrease in the Vehicle-treated group, whereas low- and medium human mesenchymal stem cell doses established a significant increase. High dose had a tendency to decreased values resulting in muscle damage and further occlusions. A dose response could not be detected.

\section{Conclusions}

This study showed that the centrifuged human bone marrow suspension containing low and medium concentrations of mesenchymal and hematopoietic stem cells significantly improved the hind limb ischemia as compared to vehicle-treated group in T-cell deficient rats, and that this effect is almost lost with higher doses, maybe due to hyper viscosity. 


\section{Key Words}

Critical Limb Ischemia; autologous stem cells; proof of concept; re-vascularisation

\section{Introduction}

Angiogenesis is essential for the function of most organs. The angiogenesis after organ injury or transplantation is key to the survival, regeneration and functioning of the affected organ. Stem cell transplantation was established to induce promotion of repair of ischemic tissue partially through angiogenesis which has been shown in models of hind limb ischemia for example (1). Stem cell therapies differ with respect to the origin of the cells, cell numbers and route of application (2) (3) $(4,5)$. Studies were for example performed with unfractionated bone marrow cells, endothelial progenitor cells and/or peripheral blood mononuclear cells which make comparison very difficult $(3)(4,5)$. In addition, the different cell types have different properties and functions. A recent meta-analysis could not identify an approach that is clinically established neither with respect to cell type, dose route of application (6). Mesenchymal stem cells offer a unique combination of anti-inflammatory and regenerative effects whereas endothelial stem cells may directly promote angiogenesis (7). In animals with subacute ligated femoral arteries, neo-angiogenesis with restoration of the blood flow to the ischemic limb was reported to develop after injection of different kinds of stem cells (8-15). These findings are considered to be the effect of both cell presence and the paracrine secretion of growth factors and cytokines. The role of the various cell types of the transplants (mesenchymal cells, hematopoietic cells, etc.), however, remains unclear, although after transplantation, both the number of circulating endothelial progenitor cells increases $(16,17)$. Some cells of the transplant were found to be incorporated into newly formed collaterals in ischemic areas (18). However, the intra-arterial versus the intramuscular route was found to be equivalent (6) which raises questions about the mechanisms since no direct initial contact of the transplanted cells with the injured tissue was apparently necessary (2). Although a systemic effect may be suspected, there was however a dose dependency in response (2).

We asked in this study whether we can determine an optimal dose for the administration of unfractionated human bone marrow derived cells, containing a mixture of mesenchymal, hematopoietic stem cells and angiotrophic factors after centrifugation, in an 
immunodeficient animal model in order to promote angiogenesis after hind limb ischemia. We combined intraarterial with intramuscular administration of human cells to test for enhanced revascularization after 35 days in a dose dependent manner.

\section{Methods}

The animal experiments were executed after animal approval was obtained. We chose an immunodeficient animal model which does not mandate the concomitant administration of immunosuppressive drugs in order to avoid confounding.

After clinical examination, 35 female animals were anesthetized and the right A. iliaca externa was proximally occluded to establish a right hind limb ischemia. Rats were anesthetized with isoflurane, and the right hind limb was shaved with an electric clipper, and disinfected with ethanol. The animals then were subcutaneously injected with tramadol 2 $\mathrm{mg} / \mathrm{kg}$ body weight (BW) for analgesia. After an incision in the right hind limb, the A. iliaca externa was exposed and proximally occluded with Vycril 5-0. Subcutaneous tissue was then sutured with Vycril 3-0 and the skin was closed with Michel clamps. After the occlusion, the animals were kept one week in their cages for recovery before digital subtraction angiography.

Seven days after the occlusion, the animals were again anesthetized with isoflurane and then the ventral region of the neck was clipped and disinfected with ethanol. The animals received Tramadol $2 \mathrm{mg} / \mathrm{kg}$ BW subcutaneously for analgesia. First, a conventional X-Ray was performed to document the anatomic locations for further analysis. Secondly, the Arteria carotis communis sinistra (ACCS - left common carotid artery) was prepared and a catheter (1270.02 Umbilical Catheter, Vygon, 2,5 French $30 \mathrm{~cm}$ ) was inserted into the ACCS and guided into the aorta, with the catheter tip ending directly in front of the bifurcation of the A. iliaca interna. Finally, a Digital Subtraction Angiography (DSA) was performed with a GE OEC 7700 X-ray device and using 0.3 to $0.5 \mathrm{ml}$ of Ultravist ${ }^{\circledR}$ in a fast bolus to visualize the vessels of the rats. The complete DSA procedures were repeated at day 35 after the occlusion with the difference that the catheters were then inserted directly in the abdominal aorta. The DSA device (GE, OEC 7700) settings were 2 images per second at 1,1-1,2 mA and $52-54 \mathrm{mV}$. The analysis of the angiography was performed using Image $J^{\circledR}$ software. 


\section{Cell preparation}

Six healthy volunteers were recruited for iliac crest-bone marrow collection, after giving written informed consents. Bone marrow samples were processed under good manufacturing practice (GMP) conditions by centrifugation according to standard operating protocols to obtain the buffy coat (internal product name ASCT01). The samples were analyzed to assess the total number of viable $\mathrm{CD} 34^{+}$cells. Within 48 hours after the bone marrow procurement, the samples were administered to the rats. During the whole procedure, bone marrow cells were kept between 2-8 degrees Celsius, which ensured a cellular viability greater than $95 \%$ (data not shown). Directly before administration to the rats, bone marrow cell suspensions were carefully resuspended and adjusted with sodium chloride $\mathbf{0 . 9 \%}$ (vehicle) solution to the needed concentrations. Table 1 gives for each group the mean number of administrated viable $\mathrm{CD} 34^{+}$cells per $\mathrm{kg}$. Study drugs (bone marrow cell suspension and vehicle) were injected both intraarterial (through the DSA catheter over a period of $10 \mathrm{~min}$ ) and intramuscular through syringes, warmed in the water bath at $37^{\circ} \mathrm{C}$ prior to injection. The ratio of cells injected intraarterially and intramuscularly were 2:1. Cell concentrations for intraarterial injections are given in Table 1.

The primary outcome of this study was the change in the vascular density of the blood vessels as an indicator of angiogenesis in the ischemic limbs after treatment with various doses of bone marrow cells versus treatment with the vehicle. Therefore, regions of interest (ROI) were set by conventional X-ray from the head of the femur to the distal end of the fibula of both the vascular occluded right and the intact left hind limbs of the individual rats. In these regions, the mean gray value (MGV) was visualized in the digital images taken during the subtraction angiography (DSA) at day 7 and day 35 (MGV-7 and MGV-35). MGV was calculated using the pixel calculator of the Image $J^{\circledR}$ Software.

The effect of the unilateral ligation of the (right) iliac artery was studied by comparing MGV7 of both legs. Then, the effect of the vehicle on vascularization in the right hind limb after ligation was studied by comparing Vehicle MGV-7 and MGV-35 calculations in the right hind limb. The effect of bone marrow cells on the vascularization in the ischemic legs was studied by comparing their MGV-7 and MGV-35 calculations in the ligated right hind limbs with the vehicle MGVs. 


\section{Statistical Analysis}

The changes in MGV for the different bone marrow cells treated groups were tested to the changes in the MGV for the Vehicle group using analysis of variance (ANOVA).

The individual changes between the MGV at day 35 and day 7 for every group were tested within the group using a pair wise T-test. At last, the positive changes in the MGV ratio's for the occluded legs are grouped as 1 . No changes or negative changes are grouped as 0 . With a Chi-square non parametric test the bone marrow treated group was compared with the Vehicle group and tested for significance. Statistical significance was assumed with $p<0.05$ levels.

\section{Results}

The surgery was successfully performed in all animals. There was no difference in body weight between the different treatment groups and the vehicle group before the surgery (data not shown). In the treatment group treated with the high bone marrow cell dose, two animals died during the application of this dose, and another one had to be sacrificed during follow-up. During the follow-up of the treated animals, all remaining animals showed an uncomplicated and expected body weight development (data not shown). During the study the limb temperatures were measured. The temperature of the limb was stable over time (data not shown).

The MGV-7 and MGV-35 for both vascular occluded right and intact left legs were calculated for each animal. There was a significant decrease in MGV-7 in the occluded right legs as compared with the intact left legs in MGV-7 (figure 1a), which showed about $40 \%$ loss of calculated vascular density as compared to the left MGV. During the follow-up, vehicletreated animals showed significant progression up to roughly $60 \%$ of the MGV at day 35 (figure $1 \mathrm{~b}$ ) which is in line with results in immunocompetent animal models (8).

At day 35 , a significant increase of vascular density was found in all other bone marrow cells treated groups compared to the vehicle treated group (figure 1b) except in the high dose group. To set these changes into proportion to each other, figure $1 \mathrm{c}$ presents the percentages of change in MGV at day 35 for the occluded leg for the different ASCTO1 and vehicle treated groups. Low and medium dose of unfractionated bone marrow cells improved mean gray values significantly which high dose treatment did not. The 
proportional improvement was highest in the low dose bone marrow cells group. We then asked the question whether there was a correlation between the number of $\mathrm{CD}_{3} 4^{+}$cells and the MGV improvement. Figure 2 represents the dose response curve which showed no clear correlation between the absolute number of $\mathrm{CD}_{3} 4^{+}$cells administered intra-arterially and intramuscularly and the absolute change in MGV at day 35 compared with day 7.

The concomitant application of cells intra-arterially and intramuscularly into the affected limb was done to optimize the re-vascularisation. Angiographical data, as obtained during this study, illustrated that the newly formed vessels were still very small. A representative study is shown in Figure 3.

\section{Discussion}

We used for our study a T cell deficient rat model in order to avoid confounding effects of immunosuppressive drugs. The critical limb ischemia did deteriorate in the vehicle group, which is in line with the natural course of the disease. In order to promote the effects of the unfractionated bone marrow preparation we tested the combined intraarterial/intramuscular treatment with human bone marrow-derived stem cells in the ischemic leg, at day 35 in a dose dependent manner. We found no dose dependency but a profound clinical improvement after the lower and medium cell concentrations with a significant increase of vascular density, if compared with the reduced vascular density at day 7 . This significant repair of vascular density, though, was not seen in the animals treated with higher bone marrow cell concentrations. Treating the ligated legs with these high doses, on the contrary, had a non-significant tendency to further loss of vascular density as compared to the MGV-7 situation was established. We tested in 5 animals the highest possible cell concentration of bone marrow cells which resulted in the death of 2 out of 5 rats during administration of this preparation. Although in general, bone marrow cell treatment was well tolerated in the low and medium dose groups, in the higher dose groups there were some difficulties with the intra-arterial administration, due to the high viscosity of the solutions, probably resulting in physical obstruction in these vessels despite the rather long infusion period.

Angiographic data illustrated that the newly perfused vessels were still very small (Figure 4). This could indicate that our intra-arterial and intramuscular approach did not generate an 
expedition of the underlying angiogenesis. The process of angiogenesis is starting up slowly, making the clinical improvements initially difficult to detect. Therefore, a longer follow up period after treatment would probably result in more and longer blood vessels formation and diameter in the treatment groups. This is most likely not possible in the placebo group due to the natural deterioration of the hind limb occlusion.

Our approach of intra-arterial plus intramuscular administration was very different from currently clinically tested intramuscular or intra-arterial approaches (7) (6). We chose the combined approach to bring the cells to the circulation and allow access from the endothelial side for cell to cell interaction and secretion of paracrine factors (19) (20). In addition, the allogenic cells have a short half-life irrespective of the immunosuppression of the animals (21). The chosen xenograft model, in which T-cell deficient rats were treated with human bone marrow stem cells without any further immune suppression, is useful in order to avoid the confounding effects of immunosuppression (22). It has the advantages, such as better animal treatment conditions, and better mimicking the human clinical situation when compared with studies were immune suppressive drugs were used.

It would be interesting to also assess the effect of individual treatments (intra-arterial and intra-muscular administration) to clarify the contribution of each route of stem cells administration. Since clinical trials have shown an equipoise between the two administration routes we did not include these groups in order to reduce the number of experimental animals.

We faced serious limitation due to the high viscosity which makes this approach less promising (4). Our study has additional limitations with respect to the time period of follow up and the mode of administration. The approach to test cell-based or cell-derived preparations in T-cell deficient rats is however possible and allows the assessment of cellbased or cell-derived preparations without additional drugs administration since the quest for the best cell preparation is still ongoing (21). The different properties of the cell mixture of bone marrow versus mesenchymal stromal cells offers opportunities in particular with respect to immunomodulation and/or regeneration (23). Despite the evolving evidence for clinical effectiveness of autologous bone marrow derived treatment (7), the preparation of 
bone marrow and the selection of subgroups of cells from bone marrow warrants further studies (4).

\section{Conclusion}

This study reports that $\mathrm{T}$ cell deficient rats can be used as a model for angiogenesis after critical limb ischemia. We used the immunosuppressed animals to test centrifuged human bone marrow suspension containing mesenchymal and hematopoietic stem cells which significantly improved hind limb perfusion in T cell deficient rats. This study did show that the centrifuged human bone marrow suspension containing low and medium doses of bone marrow cells significantly improved the hind limb ischemia as compared to vehicle-treated group in T-cell deficient rats, and that this effect was lost with higher doses, most likely due to hyper viscosity.

\section{Acknowledgements}

The technical excellence of Dr. med. Vet. Simone Odau is gratefully acknowledged. 


\section{Figure 1}

1a. Intact Left Leg

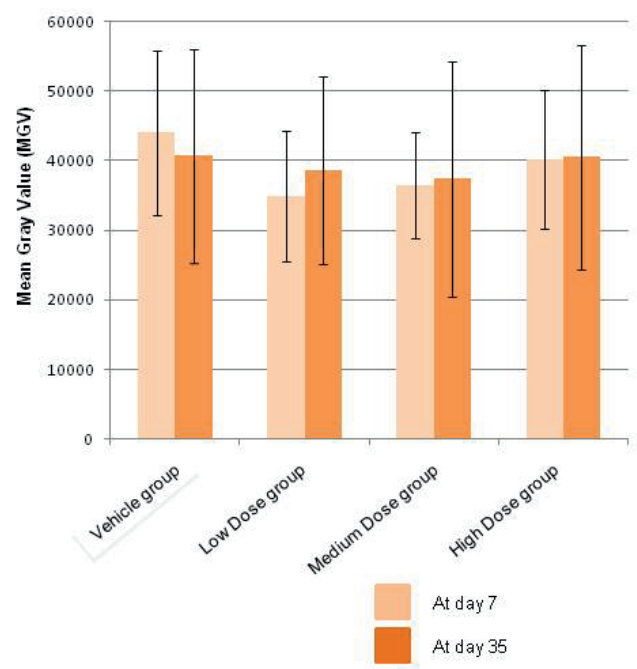

1b. Occluded Right Leg

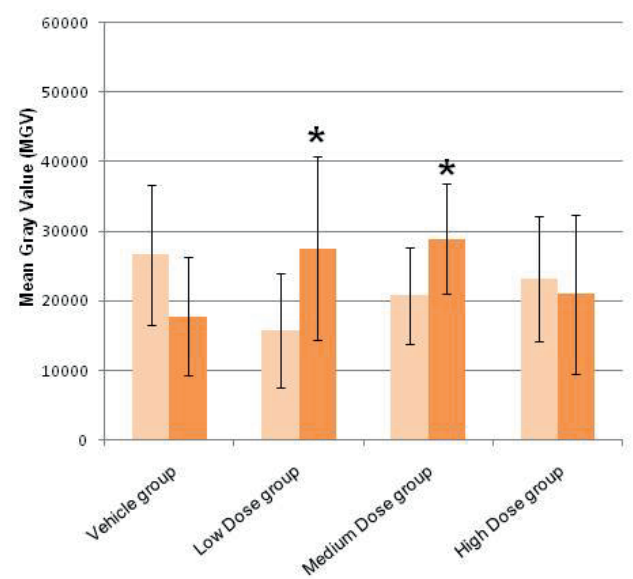

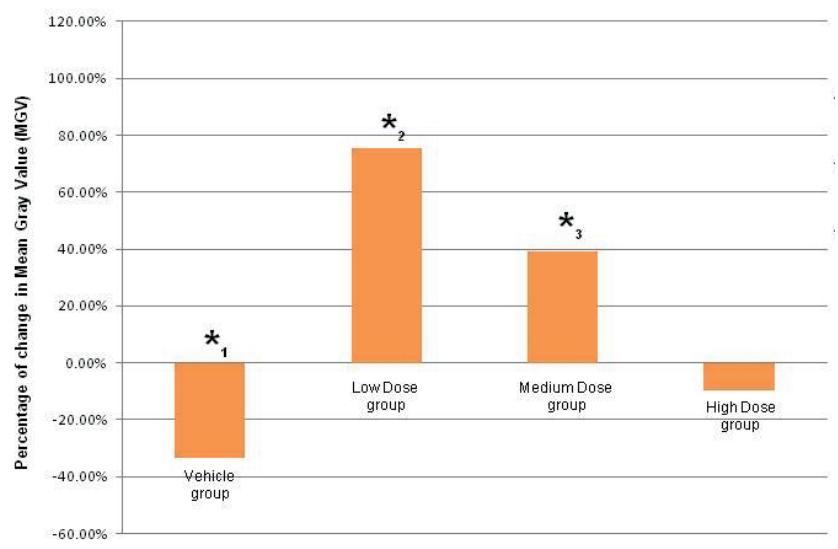

Figure 1: Overview MGV-7 and MGV-35 values in the ischemic right hind leg (left) and in the intact left hind leg. (a) MGV in intact left leg for Vehicle, low dose, medium dose and high dose group. (b) MGV in occluded right leg for vehicle, low dose, medium dose and high dose group. Significance compared to MGV-7 with ANOVA marked with ${ }^{*} p<0.01$. (c): Percentage of change between the MGV at day 7 and 35 for the occluded leg and the different groups. $\left({ }^{*} 1\right)$ Deterioration of the limb ischemia in vehicle treated animals (Pair wise T-Test $p=$ 0.003); ( $\left.{ }^{*} 2\right)$ Difference after low dose cell therapy to the vehicle treated group (ANOVA $\left.p<0.001\right) ;(* 3)$ Difference after medium dose cell therapy to the vehicle treated group (ANOVA $p<0.01$ ). No improvement after high dose therapy. 


\section{Figure 2}

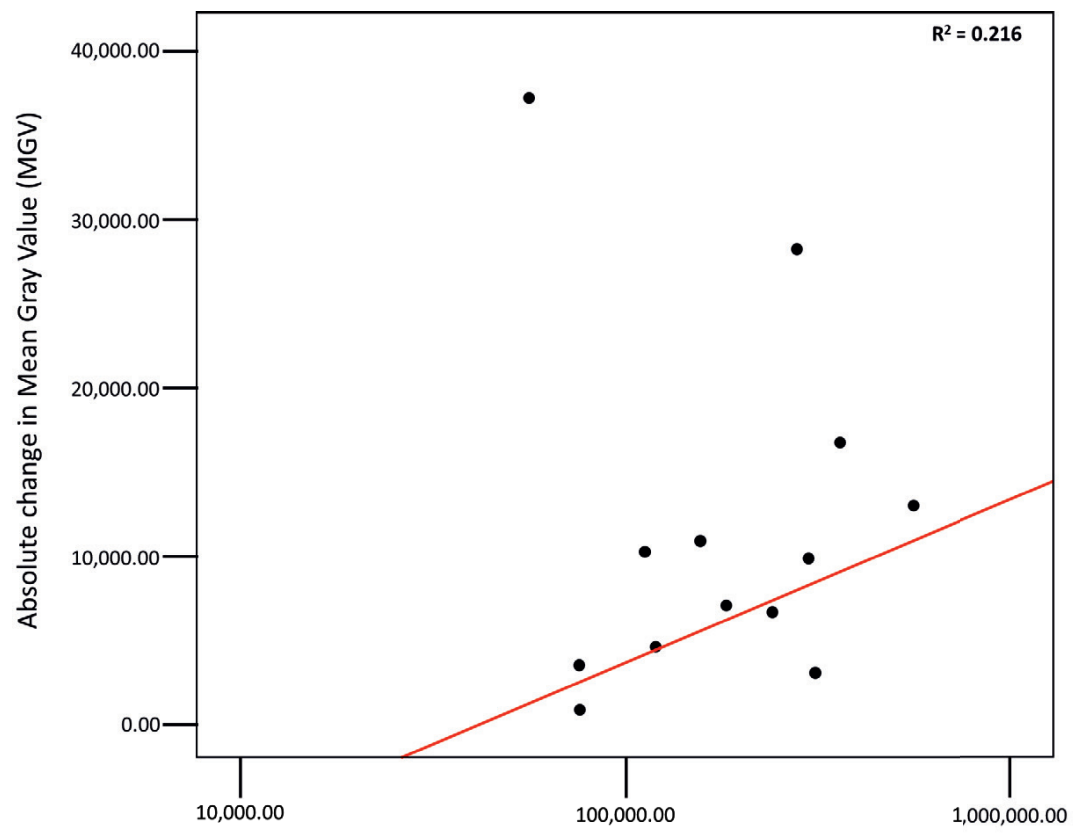

Absolute number of administered CD34+ Cells on a log-scale

Figure 2: The dose-response graph describes the relationship between the total number of $\mathrm{CD} 34^{+}$ cells in low and medium dose ASCT01 and the improvement on day 35 in Mean Gray Value (MGV). The values are weighted by dividing the expected value by the measured value for each point. The $\mathrm{R}^{2}$ is 0,216 , which means that no correlation between the total $\mathrm{CD}_{3} 4^{+}$and the Mean Gray Value was found. 


\section{Figure 3}
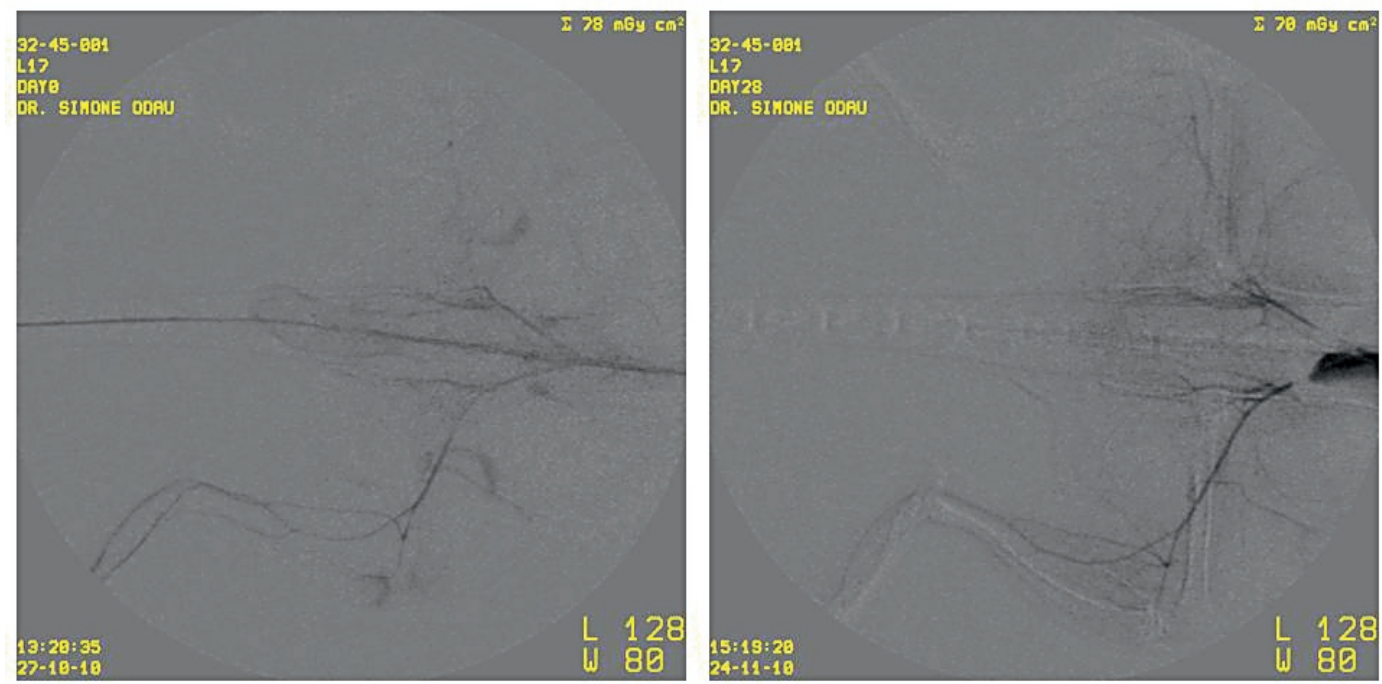

Figure 3: Example of a set of angiographic images of a with human bone marrow cells (ASCTO1) treated T cell deficient rat. The left image is taken at day 7 (before treatment) and the right image is taken at day 35 after the treatment with low dose of human bone marrow cells. Despite concomitant application of cells intramuscularly and intravenously, the newly formed vessels are very small. 
Table 1 Overview mean number of $\mathrm{CD} 4^{+}$Cells per treatment group

\begin{tabular}{|l|l|c|}
\hline Concentration group & $\begin{array}{l}\text { Mean number of viable CD34 } \\
\text { Cells administered intraarterially } \\
\text { (I.A.) and intramuscularly (I.M.) } \\
\text { per animal }\end{array}$ & Total number of rats \\
\hline Low Concentration & $\begin{array}{l}\text { I.A.: } 327,600 \text { CD34 }{ }^{+} \text {Cells } / \mathrm{kg} \\
\text { I.M.: } 163,800 \text { CD34 Cells } / \mathrm{kg}\end{array}$ & 7 \\
\hline Medium Concentration & I.A.: 688,800 CD34 $4^{+}$Cells $/ \mathrm{kg}$ & 6 \\
\hline High Concentration & I.M.: 344,400 CD34 Cells $/ \mathrm{kg}$ & 5 \\
\hline
\end{tabular}




\section{References}

1. Tateishi-Yuyama E, Matsubara H, Murohara T, Ikeda U, Shintani S, Masaki H, et al. Therapeutic angiogenesis for patients with limb ischaemia by autologous transplantation of bonemarrow cells: a pilot study and a randomised controlled trial. Lancet. 2002;360(9331):427-35.

2. Klepanec A, Mistrik M, Altaner C, Valachovicova M, Olejarova I, Slysko R, et al. No difference in intra-arterial and intramuscular delivery of autologous bone marrow cells in patients with advanced critical limb ischemia. Cell Transplant. 2012;21(9):1909-18.

3. Wang ZX, Li D, Cao JX, Liu YS, Wang M, Zhang XY, et al. Efficacy of autologous bone marrow mononuclear cell therapy in patients with peripheral arterial disease. J Atheroscler Thromb. 2014;21(11):1183-96.

4. Fadini GP, Agostini C, Avogaro A. Autologous stem cell therapy for peripheral arterial disease meta-analysis and systematic review of the literature. Atherosclerosis. 2010;209(1):10-7.

5. Scheubel RJ, Holtz J, Friedrich I, Borgermann J, Kahrstedt S, Navarrete Santos A, et al. Paracrine effects of CD34 progenitor cells on angiogenic endothelial sprouting. Int J Cardiol. 2010;139(2):134-41.

6. Abdul Wahid SF, Ismail NA, Wan Jamaludin WF, Muhamad NA, Abdul Hamid MKA, Harunarashid $\mathrm{H}$, et al. Autologous cells derived from different sources and administered using different regimens for 'no-option' critical lower limb ischaemia patients. Cochrane Database Syst Rev. 2018;8:CD010747.

7. Ponemone V, Gupta S, Sethi D, Suthar M, Sharma M, Powell RJ, et al. Safety and Effectiveness of Bone Marrow Cell Concentrate in the Treatment of Chronic Critical Limb Ischemia Utilizing a Rapid Point-of-Care System. Stem Cells Int. 2017; 2017:4137626.

8. Kinnaird T, Stabile E, Burnett MS, Epstein SE. Bone-marrow-derived cells for enhancing collateral development: mechanisms, animal data, and initial clinical experiences. Circ Res. 2004;95(4):354-63.

9. Schatteman GC, Hanlon HD, Jiao C, Dodds SG, Christy BA. Blood-derived angioblasts accelerate blood-flow restoration in diabetic mice. J Clin Invest. 2000;106(4):571-8.

10. Pesce M, Orlandi A, lachininoto MG, Straino S, Torella AR, Rizzuti V, et al. Myoendothelial differentiation of human umbilical cord blood-derived stem cells in ischemic limb tissues. Circ Res. 2003;93(5):e51-62.

11. Hirata K, Li TS, Nishida M, Ito H, Matsuzaki M, Kasaoka S, et al. Autologous bone marrow cell implantation as therapeutic angiogenesis for ischemic hindlimb in diabetic rat model. Am J Physiol Heart Circ Physiol. 2003;284(1):H66-70.

12. Kinnaird T, Stabile E, Burnett MS, Lee CW, Barr S, Fuchs S, et al. Marrow-derived stromal cells express genes encoding a broad spectrum of arteriogenic cytokines and promote in vitro and in vivo arteriogenesis through paracrine mechanisms. Circ Res. 2004;94(5):678-85.

13. Lian $\mathrm{Q}$, Zhang $\mathrm{Y}$, Zhang J, Zhang $\mathrm{HK}, \mathrm{Wu} X$, Lam FF, et al. Functional mesenchymal stem cells derived from human induced pluripotent stem cells attenuate $\operatorname{limb}$ ischemia in mice. Circulation. 2010;121(9):1113-23.

14. Lawall $\mathrm{H}$, Bramlage $\mathrm{P}$, Amann B. Stem cell and progenitor cell therapy in peripheral artery disease. A critical appraisal. Thromb Haemost. 2010;103(4):696-709.

15. Urbich C, Dimmeler S. Endothelial progenitor cells: characterization and role in vascular biology. Circ Res. 2004;95(4):343-53.

16. Shintani S, Murohara T, Ikeda H, Ueno T, Honma T, Katoh A, et al. Mobilization of endothelial progenitor cells in patients with acute myocardial infarction. Circulation. 2001;103(23):2776-9.

17. Takahashi T, Kalka C, Masuda H, Chen D, Silver M, Kearney M, et al. Ischemia- and cytokineinduced mobilization of bone marrow-derived endothelial progenitor cells for neovascularization. Nat Med. 1999;5(4):434-8.

18. Asahara T, Masuda H, Takahashi T, Kalka C, Pastore C, Silver M, et al. Bone marrow origin of endothelial progenitor cells responsible for postnatal vasculogenesis in physiological and pathological neovascularization. Circ Res. 1999;85(3):221-8. 
19. Kinnaird T, Stabile E, Burnett MS, Shou M, Lee CW, Barr S, et al. Local delivery of marrowderived stromal cells augments collateral perfusion through paracrine mechanisms. Circulation. 2004;109(12):1543-9.

20. Sahoo S, Klychko E, Thorne T, Misener S, Schultz KM, Millay M, et al. Exosomes from human CD34(+) stem cells mediate their proangiogenic paracrine activity. Circ Res. 2011;109(7):724-8.

21. Schiattarella GG, Perrino C, Magliulo F, Carbone A, Bruno AG, De Paulis M, et al. Physical activity in the prevention of peripheral artery disease in the elderly. Front Physiol. 2014;5:12.

22. Amann B, Luedemann C, Ratei R, Schmidt-Lucke JA. Autologous bone marrow cell transplantation increases leg perfusion and reduces amputations in patients with advanced critical limb ischemia due to peripheral artery disease. Cell Transplant. 2009;18(3):371-80.

23. Aurora $A B$, Olson EN. Immune modulation of stem cells and regeneration. Cell Stem Cell. 2014;15(1):14-25. 


\section{CHAPTER 6}

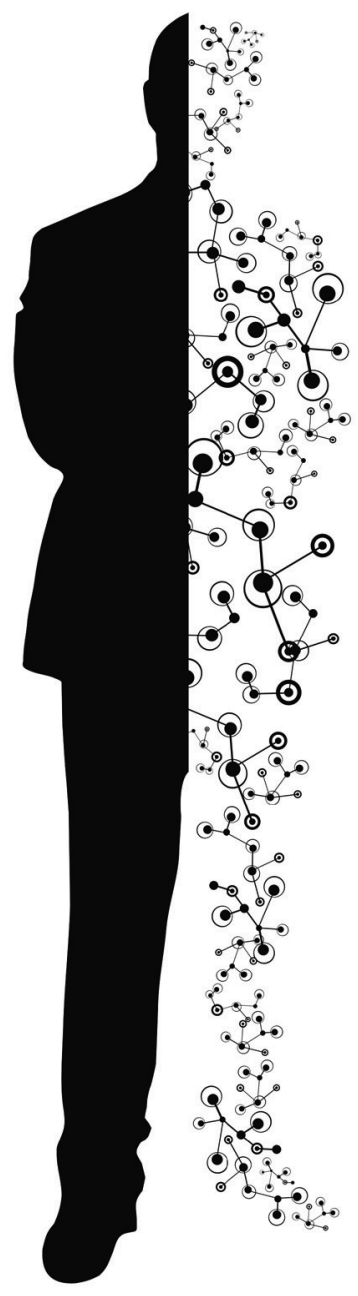




\section{Electrical stimulation promotes the angiogenic potential of adipose-derived stem cells}

Beugels J, Molin DGM, Ophelders DRMG, Rutten T, Kessels L, Kloosterboer N, Grzymala AAP, Kramer BWW, van der Hulst RRWJ, Wolfs TGAM 


\section{Abstract}

Autologous fat transfer (AFT) is limited by post-operative volume loss due to ischemiainduced cell death in the fat graft. Previous studies have demonstrated that electrical stimulation (ES) promotes angiogenesis in a variety of tissues and cell types. In this study we investigated the effects of ES on the angiogenic potential of adipose-derived stem cells (ASC), important progenitor cells in fat grafts with proven angiogenic potential. Cultured human ASC were electrically stimulated for 72 hours after which the medium of stimulated (ES) and non-stimulated (control) ASC was analysed for angiogenesis-related proteins by protein array and ELISA. The functional effect of ES on angiogenesis was then assessed in an in vivo model.

Nine angiogenesis-related proteins were detected in the medium of electrically (non-) stimulated ASC and were quantified by ELISA. The pro-angiogenic proteins VEGF and MCP-1 were significantly increased following ES compared to controls, while the anti-angiogenic factor Serpin E1/PAI-1 was significantly decreased. Despite increased levels of antiangiogenic TSP-1 and TIMP-1, medium of ES-treated ASC significantly increased vessel density, total vessel network length and branching points in chorio-allantoic membrane assays. In conclusion, our proof-of-concept study showed that ES increased the angiogenic potential of ASC both in vitro and in vivo.

\section{Introduction}

Autologous fat transfer (AFT; also called fat grafting or lipofilling) is a widely used reconstructive and aesthetic procedure, wherein fat is harvested as an injectable filler to augment or reconstruct tissue in all regions of the body ${ }^{1}$. Currently, post-operative volume loss, described to be ranging from 40 to $80 \%$ of the initial graft volume ${ }^{2-4}$, is a significant limitation of $\mathrm{AFT}$, and often requires additional grafting procedures. A lack of blood flow through the graft necessitates the transplanted cells to fully rely on the diffusion of oxygen and nutrients from surrounding tissue for their survival ${ }^{5}$. In an experimental mouse model, Kato et al ${ }^{6}$ have shown that only adipocytes in the outermost layer of the transferred fat tissue survive the first week post-operative, while adipocytes present inwards of the diffusion limit of approximately $300 \mu \mathrm{m}$ quickly die. 
Numerous studies have focused on revascularization of the graft by stimulating angiogenesis in order to improve graft survival and maximize the retained graft volume. Approaches primarily focused on increasing the vascularization of the recipient site pre- and postoperatively ${ }^{7,8}$ or on modifying the pro-angiogenic potential of the adipose graft itself by adding single growth factors (i.e. FGF-2, PDGF and IL-8) ${ }^{9-11}$, platelet-rich plasma ${ }^{12,13}$, by adding regenerative cells from stromal vascular fractions ${ }^{3,4}$ or by adding isolated adiposederived stem cells ${ }^{14,15}$. While administration of angiogenic growth factors has been proven to stimulate angiogenesis ${ }^{16}$, clinical implementation is hampered by the short serum halflife and adverse effects associated with continuous administration ${ }^{17}$. Others have suggested to enrich fat grafts with a heterogeneous population of regenerative cells, called the stromal vascular fraction, which is normally present in adipose tissue ${ }^{18,19}$. This procedure has been dubbed cell-assisted lipotransfer $(\mathrm{CAL}){ }^{19}$. However, Peltoniemi et al. ${ }^{20}$, did not find CAL to be superior to non-enriched fat grafts in respect to graft volume retention and for this reason concluded that regular AFT is cheaper, faster and has a lower risk of contamination. Adipose-derived stem cells (ASC) being abundantly present in adipose tissue are part of this stromal vascular fraction and have been studied for their angiogenic properties under numerous conditions ${ }^{14,15,21-25}$. In the only human clinical trial so far applying pure ASC, the authors isolated ASC from aspirated fat and expanded them in vitro for enriching the fat grafts that were injected in a second procedure ${ }^{26}$. Although ASC-enriched fat grafts remarkably retained $80.9 \%$ of the original graft volume (versus $16.3 \%$ for the controls), incorporating ex vivo expansion in daily clinical practice remains difficult because of its time and labour intensiveness, associated high costs and regulatory issues. Taking above mentioned limitations into account, a potential treatment which increases graft volume retention by stimulating revascularization in a cost-effective, non-invasive way is therefore essential to improve clinical care.

Previous in vitro and in vivo studies have demonstrated that electrical stimulation (ES) is able to stimulate angiogenesis in a variety of tissues and cell types ${ }^{27-32}$. Sheikh et al demonstrated for example that ES of ischemic rabbit hind limbs significantly induced both arteriogenesis and angiogenesis ${ }^{27}$. The exact mechanisms remain to be elucidated, although in endothelial cells the enhanced release of VEGF and activation of VEGF receptors (VEGFRs), phosphatidylinositol-3-kinase (PI3K)-Akt and Rho-ROCK elements of the VEGFR signalling pathway play a significant role ${ }^{29}$. A major advantage of ES is that by influencing the 
secretion profile of stimulated cells it can induce autologous cells to secrete a myriad of important factors, in contrast of having to inject specific exogenous factors ${ }^{30}$. In light of these findings, we hypothesized that ES would enhance the secretion of paracrine angiogenic factors by ASC, leading to increased angiogenesis. In this study we aim to determine whether ES of ASC can stimulate in vivo parameters of angiogenesis.

\section{Results}

\section{Determination of the electrical stimulus parameters}

A titration experiment was performed to analyse the effect of different electrical stimulation parameters (i.e. voltage, pulse duration and frequency) on the viability and detachment of the ASC after 72 hours of continuous stimulation. Starting with $4 \mathrm{~V} / \mathrm{cm}, 6 \mathrm{~ms}$ pulses at a frequency of $2 \mathrm{~Hz}$, one parameter per experiment was increased or decreased $(n=3)$. High viability of the cells was detected for a maximum voltage of $4 \mathrm{~V} / \mathrm{cm}$ and maximum pulse duration of $6 \mathrm{~ms}$. Thereafter viability decreased versus unstimulated control (Fig. 1). The decrease in viability was paralleled by cell detachment in culture. Frequency had a lower impact on viability and a decreased viability was only seen when the frequency was set to the device's maximum $(25 \mathrm{~Hz})$.

A

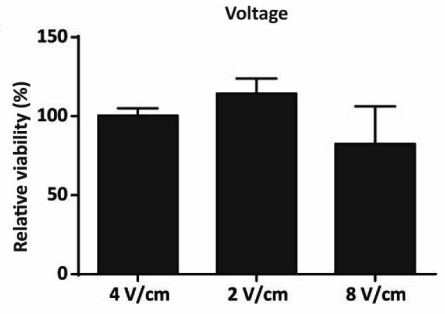

C

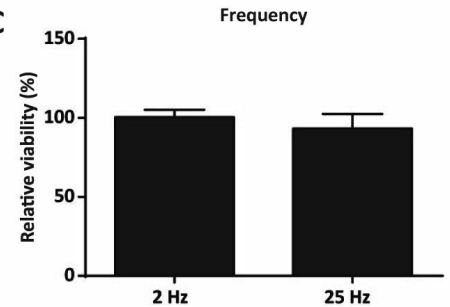

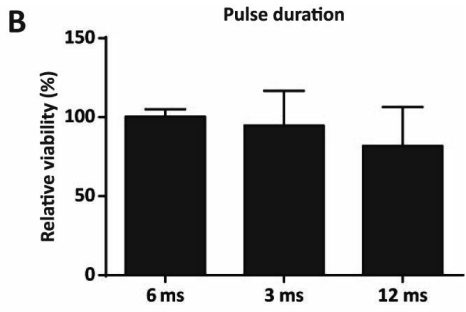

Figure 1. Determination of relative cell viability after electrical stimulation. Relative cell viability of electrically stimulated ASC for 72 hours versus unstimulated controls (CTRL set at $100 \%$ ). Starting with a protocol of $4 \mathrm{~V} / \mathrm{cm}, 6 \mathrm{~ms}$ pulses and $2 \mathrm{~Hz}$, one parameter was changed per experiment while 
the other two parameters were kept at the original level. (A): Voltage was increased and decreased twofold. $4 \mathrm{~V} / \mathrm{cm}$ had no effect on cell viability, whereas $2 \mathrm{~V} / \mathrm{cm}$ increased viability and $8 \mathrm{~V} / \mathrm{cm}$ decreased viability. (B): Pulse duration was set at $6 \mathrm{~ms}, 3 \mathrm{~ms}$ and $12 \mathrm{~ms}$. Where 6 and $3 \mathrm{~ms}$ had no effect on cell viability, $12 \mathrm{~ms}$ decreased cell viability. (C): Only frequencies of more than tenfold the starting value showed a decrease in viability. Values are expressed as the mean percentage of relative viability (of ES vs. CTRL) + SD. $n=3$ per parameter.

An additional experiment was conducted to investigate the effect of different electrical stimulation parameters on the concentration of VEGF $A$ in the conditioned medium as a key component of angiogenic process (Fig. 2). Using $4 \mathrm{~V} / \mathrm{cm}, 6 \mathrm{~ms}$ pulse duration and $2 \mathrm{~Hz}$ we found a 1.85 times increase in VEGF concentration compared to non-stimulated control. Elevated levels of VEGF were also found when we increased voltage (factor 1.48 at $8 \mathrm{~V} / \mathrm{cm}$ ), pulse duration (factor 2.59 at $12 \mathrm{~ms}$ ) and frequency (factor 10.41 at $25 \mathrm{~Hz}$ ). However, in case of the elevated voltage and pulse duration groups, cells were substantially detached which was even in part reflected by increased cell death. This phenomenon was especially prevalent when ASC were exposed to the highest frequency, explaining the relatively high VEGF concentration in this group. When the pulse duration was decreased to $3 \mathrm{~ms}$, a 1.92 times higher VEGF concentration was found when compared to unstimulated cells. However, the inter group variation between concentrations was higher when compared to the cells exposed to $4 \mathrm{~V} / \mathrm{cm}, 6 \mathrm{~ms}, 2 \mathrm{~Hz}$. Importantly this latter protocol was also used as preferred setting in a previous publication. ${ }^{33}$ Based on the group homogeneity and historical data, the parameters $4 \mathrm{~V} / \mathrm{cm}, 6 \mathrm{~ms}$ and $2 \mathrm{~Hz}$ were used as standard setting for the study. 


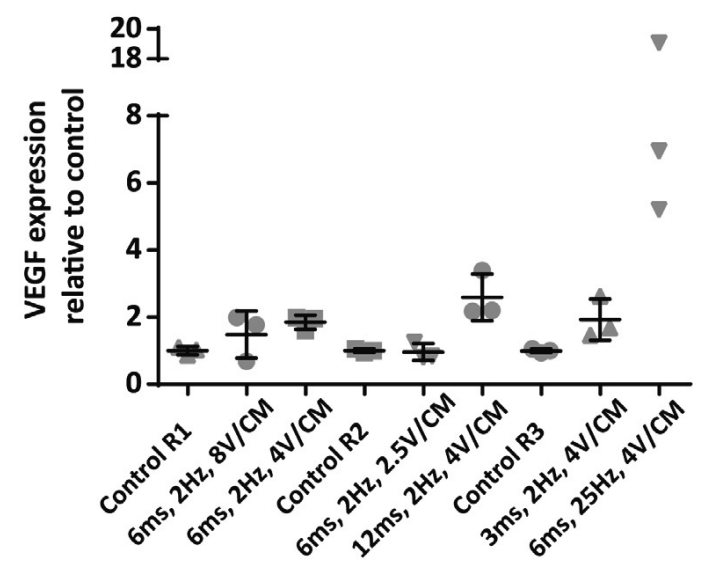

Figure 2. Three stimulation runs, each containing two ES groups and a control, were performed to analyse the effect of different treatment settings. Using $4 \mathrm{~V} / \mathrm{cm}, 6 \mathrm{~ms}$ pulse duration and $2 \mathrm{~Hz}$ a 1.85 times increase in VEGF concentration was found compared to nonstimulated control. Elevated levels of VEGF were also found when we increased/decreased voltage. In the elevated pulse duration and frequency groups, cells were substantially detached which was in part reflected by increased cell death, especially in the $25 \mathrm{~Hz}$ group, explaining the highly increased VEGF A expression. Values are expressed as the mean VEGF expression relative to control. $\mathrm{N}=3$ per stimulation protocol.

\section{Conditioned medium of electrically stimulated adipose-derived stem cells contains multiple pro- and anti-angiogenic factors.}

Medium of both ES and CTRL ASC cultures for time points $0 \mathrm{~h}$ (baseline) and $72 \mathrm{~h}$ were screened for the presence of pro- and anti-angiogenic factors using a human angiogenesis proteome profiler array (Fig. 3A), which has been used for ASC before ${ }^{34}$. The presence of 13 separate proteins was detected in the conditioned medium of ES and CTRL 72h, of which 9 proteins (IGFBP-3, IL-8, MCP-1, PTX-3, SERPIN-E1, SERPIN-F1, TIMP-1, TSP-1 and VEGF-A) were above the pixel density threshold of $1 \times 10^{3}$ arbitrary units (Fig. 3B). No difference between control and ES group in terms of baseline medium was found. Regarding the typical semi-quantitative nature of the protein profiler array, no conclusions were drawn for differential signal between the groups. Rather, the proteins above threshold were assigned for further quantitative analysis. 
A

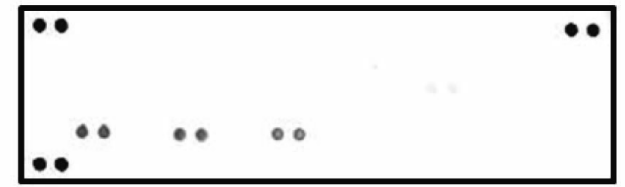

ES Oh

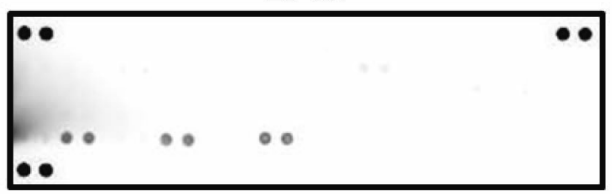

B

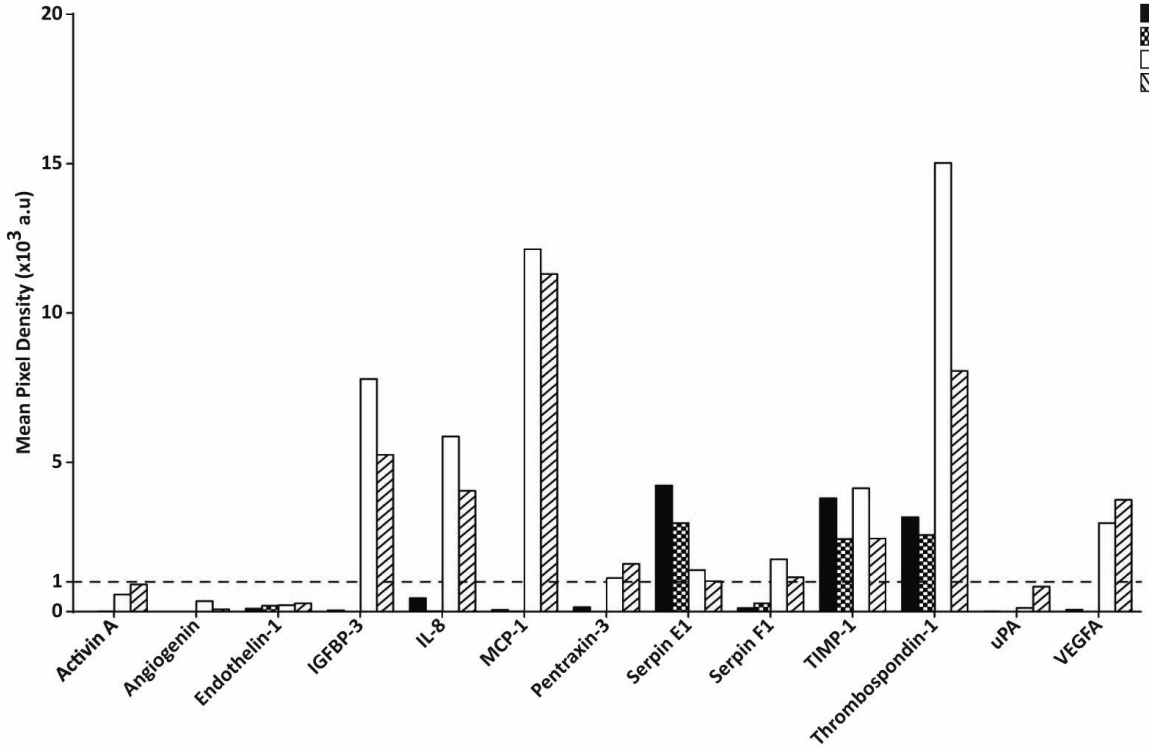

CTRL 72h

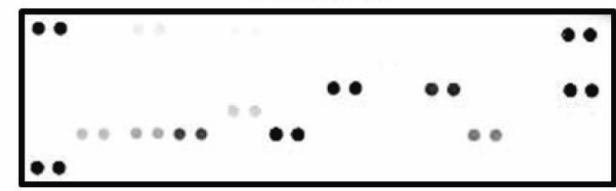

ES 72h

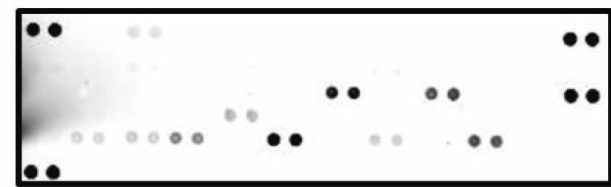

X ES Oh

CTRL 72

MVES $72 h$

Figure 3. Screening of conditioned medium using a human angiogenesis proteome profiler array. (A): the respective membranes are depicted for CTRL and ES $0 \mathrm{~h}$ and $72 \mathrm{~h}$, respectively. Next to the positive array controls in upper left, upper right and lower left corners, the in duplo spots for a total of 13 proteins were visible after scanning. (B): Mean pixel density was calculated for each spot, after normalization to the background. Nine proteins reached a minimum mean pixel density of $1 \times 10^{3}$ arbitrary units (a.u.; dotted line). For each group a single medium sample was analysed. 


\section{Electrical stimulation increases both pro- and anti-angiogenic proteins in ASC medium}

To confirm and quantify differential expression of the identified proteins, ELISA were performed on baseline $0 \mathrm{~h}$ medium and on the $\mathrm{CM}$ of $4 \mathrm{~h}-72 \mathrm{~h}$ for each of the 9 proteins. Protein levels in the $\mathrm{CM}$ showed an increase parallel to post-stimulation time, with no apparent differences between CTRL and ES for the first 48h (Fig. 4A; depicted for VEGF-A). All secreted proteins analysed revealed a similar pattern with a gradual increase in concentration over time. At 72h significant differences between ES and CTRL were detected for several proteins (Fig. 4B); VEGF A (1.99 vs. $1.30 \mathrm{ng} / \mathrm{mL}$; $p=0.04$ ), TSP-1 (1,93 v. 2,59 $\mathrm{ng} / \mathrm{mL} ; \mathrm{p}=0.03$ ), TIMP-1 (356.50 vs. $512.90 \mathrm{ng} / \mathrm{mL}$; $\mathrm{p}=0.04$ ), and MCP-1 (3.26 vs. $5.38 \mathrm{ng} / \mathrm{mL}$; $p=0.04)$ were significantly increased in the ES group vs CTRL, whereas Serpin-E1 was significantly decreased in the ES group vs CTRL (59.6 vs $88.3 \mathrm{ng} / \mathrm{mL} ; \mathrm{p}=0.03$ ). PTX-3 was increased in the ES group vs. control (7.90 vs. $12.77 \mathrm{ng} / \mathrm{mL}$ ), however the difference did not reach statistical significance $(p=0.08)$. Since only the $72 \mathrm{~h}$ medium showed significant differences in protein secretion between groups, medium of this time point was used in subsequent functional assays.

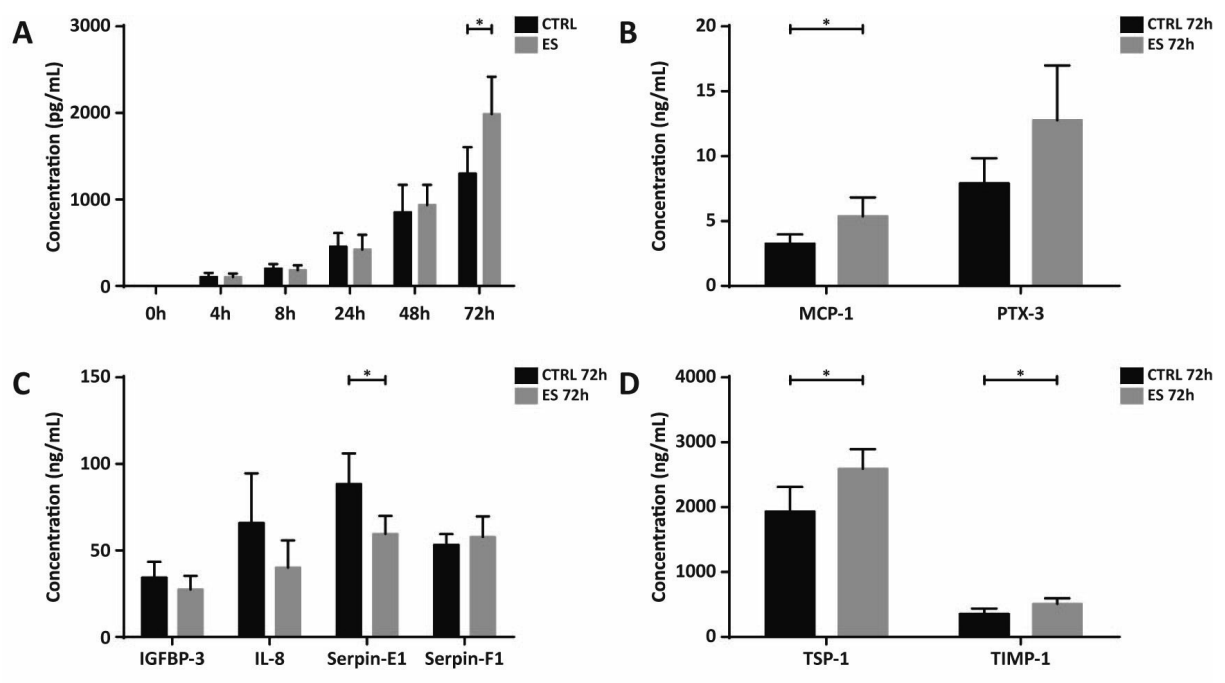

Figure 4. Quantification of the identified proteins with ELISA. (A): Concentrations of VEGF in the CM over time, ES vs. CTRL. The concentration at $72 \mathrm{~h}$ was significantly different for ES compared to CTRL $(p=0.04)$. The gradual rise in concentration visible in the time curve was representative for that of the other proteins. (B): MCP-1 was significantly increased in ES vs CTRL $(p=0.04)$, whereas the increase in PTX-3 did not reach statistical significance $(p=0.08)$. (C): Serpin-E1 was significantly decreased in ES vs CTRL ( $p=0.03)$, whereas IGFBP-3, IL-8 and Serpin-F1 were not statistically different. (D): TSP-1 and 
TIMP-1 were both significantly increased in ES vs. CTRL ( $p=0.03$ and $p=0.04$, respectively). Values displayed are mean+SD. ${ }^{*} p<0.05 . n=4$ per group.

\section{Conditioned medium of electrically stimulated ASC induced angiogenesis in vivo.}

To determine whether the observed changes in angiogenesis-related proteins after electrical stimulation induced an angiogenic effect in vivo, a CAM assay was performed. In the ES group compared to controls, a significant increase in density of the vessels within the ring was detected ( 34.7 vs $30.3 \% ; \mathrm{p}<0.05$ ), as well as an increased total vessel network length $(18,541$ vs. 16,621 px; $p<0.01)$ and a higher total number of branching points (447 vs. 380; $\mathrm{p}<0.05$ ) (Fig. 5). Representative images of the control and ES-treated CAMS, including their analysis, can be found in Supplementary Figure S1 online.

A

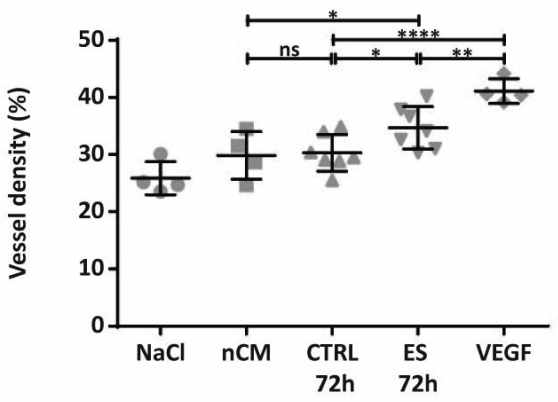

C

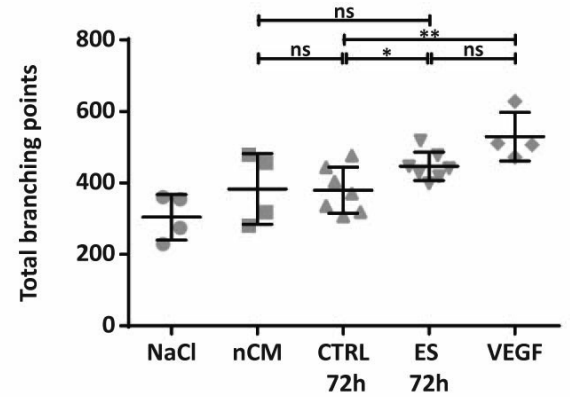

B

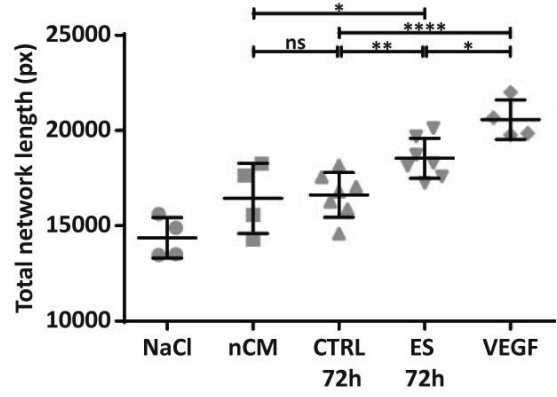

Figure 5. Conditioned medium of electrically stimulated ASC induced angiogenesis in vivo. ES CM significantly increased vessel density (A), total vessel network length (B) and total number of branching points (C) compared to CTRL CM in a chorioallantoic membrane (CAM) assay. ${ }^{*} p<0.05$; ** $\mathrm{p}<0.01 ; * * * * 0.0001 . \mathrm{n}=4-7$ CAMs per group. $\mathrm{px}=$ pixel, $\mathrm{ns}=$ not significant, $\mathrm{NaCl}=0.09 \%$ sodium chloride as negative control, $\mathrm{nCM}=$ non-conditioned baseline medium, CTRL $72 \mathrm{~h}=$ non-stimulated conditioned medium after $72 \mathrm{~h}$ of incubation, ES $72 \mathrm{~h}=$ electrically stimulated conditioned medium after 72 hours, VEGF = positive control. 
A

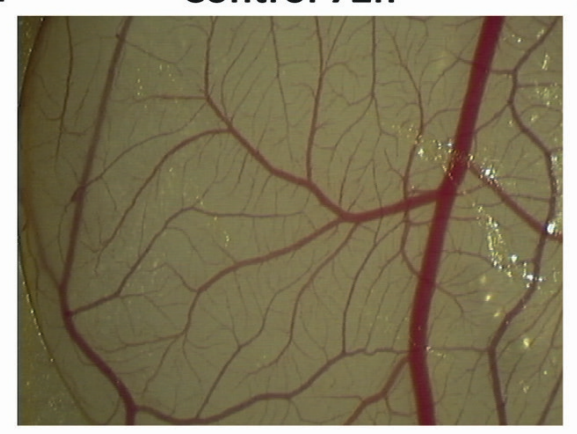

C

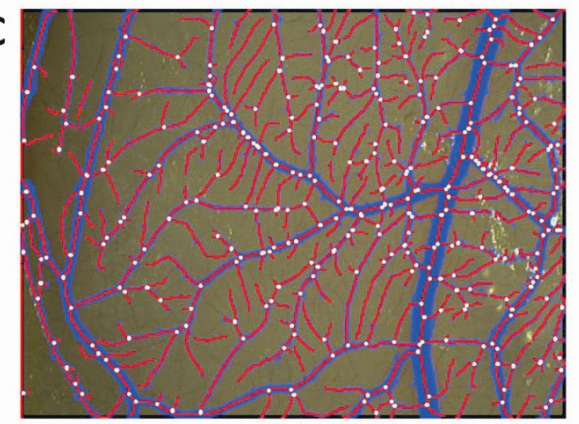

B
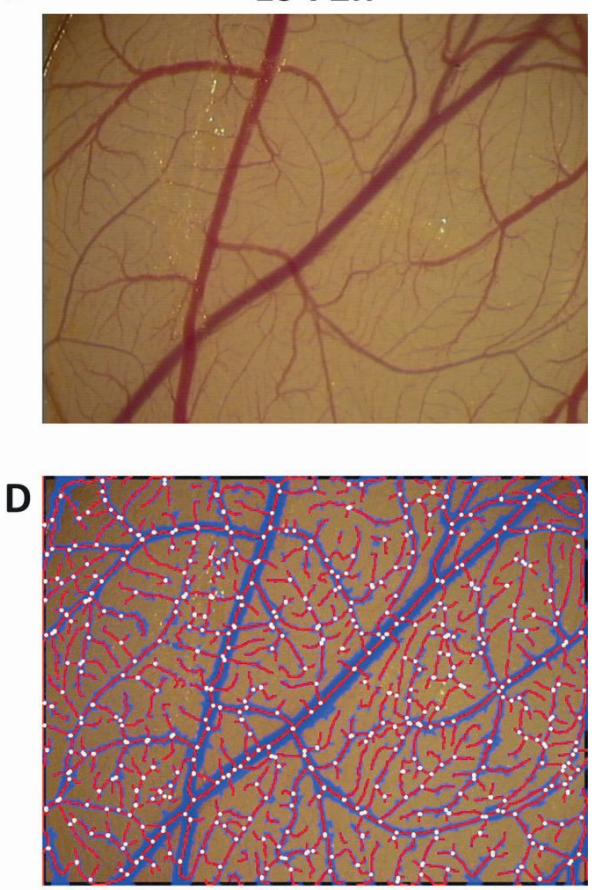

Supplementary figure S1. Representative images of the ES and CTRL CAM at day 14. (A): contrast agent was injected under the CAM to enhance the identification of capillaries before photographs were made. (B): lower panels reflect the computer analyzed overlays, wherein all vessels are tracked by automated Wimasis image analysis service.

\section{Discussion}

This proof-of-concept study demonstrates that ES of ASC improves in vitro and in vivo parameters of angiogenesis. Using a protocol of continuous stimulation with a biphasic electrical current of $4 \mathrm{~V} / \mathrm{cm}, 6$ ms pulse duration with a frequency of $2 \mathrm{~Hz}$, we detected significant differences in ASC secreted protein level for several pro- and anti-angiogenic proteins after 72 hours of ES. ES of ASC significantly augmented the concentrations of the pro-angiogenic proteins VEGF-A ${ }^{35}$ and MCP-1 ${ }^{36}$, whereas the anti-angiogenic protein Serpin E1/PAI-1 ${ }^{37}$ was significantly decreased compared to non-stimulated controls. The elevated level of VEGF confirms and extends previous work from Tandon et al. ${ }^{38}$, showing increased VEGF-A mRNA expression after ES of ASC. The concentrations of VEGF found in the conditioned medium range from an average of $1.30 \mathrm{ng} / \mathrm{mL}$ for the control group to an average of $1.99 \mathrm{ng} / \mathrm{mL}$ for the ES group, representing a $153 \%$ increase in VEGF. This increase 
seems biologically relevant since the concentrations of 1.30 and $1.99 \mathrm{ng} / \mathrm{ml}$ fit exactly in the biologically relevant range of concentrations, reported to stimulate endothelial cells in vitro 39. A robust increase of VEGF-A mRNA expression and/or VEGF-A protein level in response to ES is also supported by studies focusing on various other human cell types, such as endothelial cells ${ }^{29}$, bone marrow-derived mesenchymal stromal cells ${ }^{30}$, cardiomyocytes ${ }^{40}$, osteoblasts ${ }^{41}$ and skeletal muscle cells ${ }^{42}$. Although an influence of ES on the secretion of MCP-1 was shown for macrophages ${ }^{43}$, no reports have been made on a similar effect for ASC. Only for ASC stimulated with the pro-inflammatory stimulus TNF- $\alpha$ an increase of MCP1 secretion has been shown ${ }^{44,45}$. Comparably, TNF- $\alpha$-induced upregulation has been reported for Serpin E1/PAI-1 44,45 , whereas we provide data showing the opposite to occur after ES. Taken together, these findings suggest that ES is not the equivalent of a proinflammatory stimulus, a finding which is important for clinical translation where the amplification of the pro-inflammatory environment post-operative must be avoided. In addition to the pro-angiogenic factors described above, ES significantly induced the release of two anti-angiogenic factors, namely TSP-1 ${ }^{46}$ and TIMP-1 ${ }^{47}$. Under physiological circumstances pro- and anti-angiogenic stimuli are well balanced and related feedback mechanisms are considered to prevent unrestricted growth of blood vessels ${ }^{48}$. In this study, we found a positive pro-angiogenic effect in vivo using the conditioned medium, even though two inhibitors of angiogenesis were upregulated in the ES medium. Inhibition of TSP1 and TIMP-1 secretion in future experiments might induce an even more profound proangiogenic response.

Using the CAM assay we found a pro-angiogenic effect, as net result of the altered pro- and anti-angiogenic factors. The CAM assay allows analysis of the full blown angiogenic potential of stimuli, ranging from tubulogenesis up to stabilization of the vessel over a longer period of time. The higher cellular complexity of the CAM assay (i.e. endothelial cells and pericytes) together with the options to test the angiogenic capacity of stimuli for the different phases of angiogenesis provides in depth information on the angiogenic potential. Reviewing the results of Kim et al ${ }^{30}$ who reported a small but statistically significant increase of VEGF after electrical stimulation of BM-MSC after 1 day and a more profound effect after 2 days, we can conclude that several factors (e.g. cell type or ES protocol) influence the timeframe in which statistically significant differences between control and ES can be detected. This has important clinical implications since the majority of transplanted adipocytes in the hypoxic 
region of the graft die within the first 24 hours after transplantation ${ }^{5}$. Starting with ES of the donor and recipient site several days pre-operative in order to create a pro-angiogenic environment at the time of surgery is one way to utilize the effect of ES in the short windowof-opportunity. Alternatively, future studies should evaluate the effect of altering key stimulus parameters such as wave form, voltage, pulse duration and frequency to assess which ES protocol provides the earliest pro-angiogenic response. More precisely, Zhao et al ${ }^{49}$ found significantly elevated levels of VEGF as early as 5 minutes after ES of endothelial cells using their custom built ES set-up. Rackauskas et al ${ }^{40}$ evaluated different ES regimes for cardiomyocytes and found a frequency-dependent effect, both on the level of secreted VEGF protein in the medium and on the growth of cultured human coronary artery endothelial cells treated with CM from stimulated cells.

While various forms of ES, such as direct or alternating current (DC or $A C)^{38,50-52}$, capacitive coupling (CC) ${ }^{53}$, and pulsed electromagnetic fields (PEMF) ${ }^{54,55}$ have been used to stimulate ASC, each have their advantages and disadvantages. In case of DC stimulation, treatments can be separated in two main groups: constant or pulsed electric stimulation. Whereas the former leads to a build-up of charge causing electrolysis and hence changes in $\mathrm{pH}$ and oxygen tension around the cathode, accumulation of proteins and necrosis in vivo ${ }^{30,56}$, we chose biphasic current to circumvent these side effects due to charge balance resulting from the bidirectional wave consisting of a positive and negative phase. In addition, biphasic waveforms are often referred to as most comfortable to the patient with the least skin reaction ${ }^{57}$.

With the establishment of proof-of-concept for increased angiogenesis after stimulation of ASC, the question arises what the influence of ES would be on other angiogenic cells contained within fat. We envision a non-invasive, cost-effective and easy clinical approach to electrically stimulate adipose tissue in vivo by applying biphasic electric current from a portable stimulator through electrodes attached to the skin. Multiple treatments such as electrical muscle stimulation (EMS) and transcutaneous electrical nerve stimulation (TENS) have already used this set-up for years. Using these devices it is possible to choose from a wide range of stimuli and create an optimal treatment schedule that can include both preand post-operative stimulation, without the need to isolate cells and treat them in vitro. 
In conclusion, our proof-of-concept study showed that ES increased the angiogenic potential of ASC as demonstrated with in vitro and in vivo parameters. The results provide a valid basis for a translational study in which the effect of a non-invasive ES intervention will be analysed in a pre-clinical model of AFT.

\section{Materials and Methods}

\section{$\underline{\text { Cells }}$}

Human ASC were purchased from Lonza (Basel, Switzerland. Single donor: 52Y female, BMI 24). After thawing, ASC were seeded at a density of 5,000 cells $/ \mathrm{cm}^{2}$ in culture medium consisting of low glucose Glutamax Dulbecco's modified Eagle medium (DMEM, Thermo Fisher Scientific, MA, USA), supplemented with $10 \%$ MSC-qualified fetal bovine serum (FBS, Thermo Fisher Scientific) and 1\% penicillin/streptomycin. ASC were subcultured according to the manufacturer's protocol until reaching 90\% confluence, and used at passage 5 in all experiments.

\section{Electrical stimulation}

Cells were stimulated using the C-pace EP Cell culture stimulator (Ion-Optics Co., MA, USA) to generate a biphasic electrical current. As described previously ${ }^{33}$ cells were electrically stimulated using a protocol of $4 \mathrm{~V} / \mathrm{cm}$ with $6 \mathrm{~ms}$ pulses at a frequency of $2 \mathrm{~Hz}$. However, considering this study focused on ES of a different cell type (i.e. ASC), a dose-response curve was performed to determine the effect of different stimulation protocols on cell viability and Vascular Endothelial Growth Factor (VEGF A) release. Starting with the above mentioned parameters of $4 \mathrm{~V} / \mathrm{cm}, 6 \mathrm{~ms}$ pulses and $2 \mathrm{~Hz}$ in each subsequent stimulation round, one of the parameters (i.e. voltage) was increased or decreased, while the other two parameters were kept at the original setting. The investigated settings were as follows:

1. $2 \mathrm{~V} / \mathrm{cm}, 6 \mathrm{~ms}$ and $2 \mathrm{HZ}$

2. $4 \mathrm{~V} / \mathrm{cm}, 6 \mathrm{~ms}$ and $2 \mathrm{~Hz}$

3. $8 \mathrm{~V} / \mathrm{cm}, 6 \mathrm{~ms}$ and $2 \mathrm{~Hz}$

4. $4 \mathrm{~V} / \mathrm{cm}, 3 \mathrm{~ms}$ and $2 \mathrm{~Hz}$

5. $4 \mathrm{~V} / \mathrm{cm}, 12 \mathrm{~ms}$ and $2 \mathrm{~Hz}$

6. $4 \mathrm{~V} / \mathrm{cm}, 6 \mathrm{~ms}$ and $25 \mathrm{~Hz}$ 
ASC were cultured in 6-well plates (TC treated, Greiner bio one, Kremsmünster, Austria) at 15,000 cells $/ \mathrm{cm}^{2}$. Stimulated cell cultures were checked for cell adherence and overall morphology. After 72 hours of continuous stimulation, cell number and viability were assessed using a cell counter with integrated Trypan Blue detection (Bio-rad, CA, USA).

\section{Conditioned Medium}

Baseline medium (t0) and conditioned medium (CM) were collected each from a different well at the following time points: 4, 8, 24, 48 and 72 hours (t4-t72). To this end, ASC were seeded at 15,000 cells $/ \mathrm{cm}^{2}$ in 6 well plates in culture medium and allowed to adhere to the plate for 12 hours. Next, cells were starved by culturing them in medium with $5 \%$ serum for 12 hours and before stimulation "starvation" medium was refreshed completely. After centrifugation for 5 minutes at $300 \mathrm{~g}$ the $\mathrm{CM}$ was aliquoted and stored at $-20^{\circ} \mathrm{C}$ before use in subsequent experiments.

\section{Angiogenesis array}

To determine the production of angiogenesis-related proteins of stimulated and control samples, a human angiogenesis proteome profiler array which enables detection of 55 angiogenesis-related proteins per sample was used according to the manufacturer's instructions (R\&D Systems Inc., MN, USA). Near infrared fluorescence detection with IRDye $800 \mathrm{CW}$ (Li-cor, NE, USA) was applied and signals were visualized by using an Odyssey CLx imager (Li-cor). After normalization to the background, average pixel intensity of each of the spots' pixels within a fixed circular boundary was determined using image analysis software (Odyssey v1.2, Li-cor).

\section{Enzyme-linked immunosorbent assays}

For data confirmation of the array and quantification of the angiogenesis-related proteins with an average pixel intensity of $\geq 1.0^{3}$ arbitrary intensity units ${ }^{58}$ as defined with the angiogenesis array, enzyme-linked immunosorbent assays (ELISA) were performed. Levels of Vascular Endothelial Growth Factor A (VEGF A), urokinase-type Plasminogen Activator (UPA), Insuline-like Growth Factor Binding Protein (IGFBP-3), Interleukin 8 (IL-8), Monocyte Chemoattractant Protein 1 (MCP-1), Pentraxin-3 (PTX-3, also known as TSG-14), Plasminogen Activator Inhibitor 1 (PAl-1 also known as Serpin E1), Pigment Epithelium-derived Factor 
(PEDF, also known as Serpin F1), Thrombospondin-1 (TSP-1) and TIMP Metallopeptidase Inhibitor (TIMP-1) were measured in the CM of ES and Control groups for each respective time point, using Duoset ELISA kits (R\&D Systems Inc.) according to the manufacturer's instructions. The OD's were measured at a wavelength of $450 \mathrm{~nm}$ using a Thermo Multiskan Spectrum plate reader and Skanlt software (Thermo Fisher Scientific).

\section{Chorioallantoic membrane assay}

The protocol for the chorioallantoic membrane (CAM) assay was based on the procedure described by Le Noble et al ${ }^{59}$. Fertilized White Leghorn eggs were incubated in an egg incubator for 3 days at $37.8^{\circ} \mathrm{C}$ and a relative air humidity of $55 \%$, while being rotated every hour. At day 3 a rectangular window measuring $1 \times 1.5 \mathrm{~cm}$ was cut into the eggshell. Initially, the eggshell membrane was left intact to prevent any debris from the shell falling into the egg. After making a small hole in the eggshell membrane, two milliliters of albumin were withdrawn from the blunt end of the egg, using a $21 \mathrm{G}$ needle at an angle of 45 degrees. Care was taken to prevent sticking of the embryo against the membrane. Next, the membrane was removed to expose the embryo. Subsequently, the window was covered with adhesive tape to prevent dehydration. The eggs were placed back in the incubator without rotation until day 7 when a small silicon ring, measuring $1 \mathrm{~cm}$ and weighing $17 \mathrm{mg}$ was placed on the developing CAM. Sixty-five microliters of each respective test solution was injected inside the ring on the membrane of each egg after filtering of the solution with a 0.2 micrometer syringe filter. The test solutions consisted of sodium chloride $0.9 \%$ (negative control), nonconditioned medium ( $\mathrm{nCM}$, consisting of DMEM, 5\% FCS and 1\% PS), conditioned medium from the control group that was incubated for 72 hours (CM CTRL), conditioned medium after 72 hours of stimulation (CM ES) and VEGF (positive control, dissolved in DMEM, at a total daily dose of $20 \mathrm{ng} / \mathrm{mL}$ of VEGF). Test solutions were applied daily until day 14 . At day 14 the shell was opened to expose the CAM. In order to increase the contrast and to isolate the blood vessels in the CAM from vessels running over the yolk, a suspension of zinc oxide in vegetable oil was injected right underneath the ring and its surroundings. A Leica MS5 stereomicroscope, Leica IC A camera and VideoVelocity software (Candylabs, Vancouver, Canada) was then used to photograph the area within the ring at a magnification of $4 x$. The images were then analysed using the objective and completely automated Wimasis image analysis service (WimCAM, OnImagin Technoglogies SCA, Cordoba, Spain) ${ }^{60}$. Only eggs with 
no major blood vessels inside the ring were included, to decrease intra- and inter-group variation. Total vessel density (in \%), total vessel network length (in px) and total branching points were extracted from the analysed data.

\section{Statistical analyses}

Data are expressed as the mean \pm standard deviation (SD). ELISAs were performed in duplicate over four independent CM samples $(n=4)$. In the tube assay the $n$ value ranged from 3-5. For the CAM assay at least four eggs per condition were included $(n=4-7)$. The ELISA results were analysed by unpaired Student t-test, whereas one-way ANOVA with Bonferroni's multiple comparisons test was used to analyse the tube assay and CAM assay data (GraphPad Prism 6, GraphPad Software Inc., San Diego, USA). A p-value $<0.05$ was considered statistically significant.

Data availability

All data generated or analysed during this study are included in this published article.

AcknowledgementsThe authors wish to thank dr. Bart Vaes, Kristel Gijbels and Mick Gagliardi for their excellent technical support.

\section{Additional information}

Competing interests: the authors declare no competing interests. 


\section{References}

1 Hsu, V. M., Stransky, C. A., Bucky, L. P. \& Percec, I. Fat grafting's past, present, and future: why adipose tissue is emerging as a critical link to the advancement of regenerative medicine. Aesthetic surgery journal 32, 892-899, doi:10.1177/1090820×12455658 (2012).

2 Gir, P. et al. Fat grafting: evidence-based review on autologous fat harvesting, processing, reinjection, and storage. Plastic and reconstructive surgery 130, 249-258, doi:10.1097/PRS.0b013e318254b4d3 (2012).

3 Liu, B. et al. The adjuvant use of stromal vascular fraction and platelet-rich fibrin for autologous adipose tissue transplantation. Tissue engineering. Part C, Methods 19, 1-14, doi:10.1089/ten.TEC.2012.0126 (2013).

4 Zhu, M. et al. Supplementation of fat grafts with adipose-derived regenerative cells improves long-term graft retention. Annals of plastic surgery 64, 222-228, doi:10.1097/SAP.0b013e31819ae05c (2010).

5 Eto, H. et al. The fate of adipocytes after nonvascularized fat grafting: evidence of early death and replacement of adipocytes. Plastic and reconstructive surgery 129, 1081-1092, doi:10.1097/PRS.0b013e31824a2b19 (2012).

6 Kato, H. et al. Degeneration, regeneration, and cicatrization after fat grafting: dynamic total tissue remodeling during the first 3 months. Plastic and reconstructive surgery 133, 303e313e, doi:10.1097/prs.0000000000000066 (2014).

7 Khouri, R. K. et al. Brava and autologous fat transfer is a safe and effective breast augmentation alternative: results of a 6-year, 81-patient, prospective multicenter study. Plastic and reconstructive surgery 129, 1173-1187, doi:10.1097/PRS.0b013e31824a2db6 (2012).

8 Sezgin, B. et al. Improving fat graft survival through preconditioning of the recipient site with microneedling. Journal of plastic, reconstructive \& aesthetic surgery : JPRAS 67, 712-720, doi:10.1016/j.bjps.2014.01.019 (2014).

9 Nakamura, S. et al. Increased survival of free fat grafts and vascularization in rats with local delivery of fragmin/protamine microparticles containing FGF-2 (F/P MP-F). Journal of biomedical materials research. Part B, Applied biomaterials 96, 234-241, doi:10.1002/jbm.b.31757 (2011).

10 Craft, R. O. et al. Effect of local, long-term delivery of platelet-derived growth factor (PDGF) on injected fat graft survival in severe combined immunodeficient (SCID) mice. Journal of plastic, reconstructive \& aesthetic surgery : JPRAS 62, 235-243, doi:10.1016/j.bjps.2007.11.017 (2009).

11 Shoshani, O. et al. The effect of interleukin-8 on the viability of injected adipose tissue in nude mice. Plastic and reconstructive surgery 115, 853-859 (2005).

12 Pires Fraga, M. F. et al. Increased survival of free fat grafts with platelet-rich plasma in rabbits. Journal of plastic, reconstructive \& aesthetic surgery : JPRAS 63, e818-822, doi:10.1016/j.bjps.2010.07.003 (2010).

13 Por, Y. C. et al. Platelet-rich plasma has no effect on increasing free fat graft survival in the nude mouse. Journal of plastic, reconstructive \& aesthetic surgery : JPRAS 62, 1030-1034, doi:10.1016/j.bjps.2008.01.013 (2009).

14 Piccinno, M. S. et al. Adipose stromal/stem cells assist fat transplantation reducing necrosis and increasing graft performance. Apoptosis : an international journal on programmed cell death 18, 1274-1289, doi:10.1007/s10495-013-0878-7 (2013).

15 Lu, F. et al. Improvement of the survival of human autologous fat transplantation by using VEGF-transfected adipose-derived stem cells. Plastic and reconstructive surgery 124, 14371446, doi:10.1097/PRS.0b013e3181babbb6 (2009).

16 Walder, C. E. et al. Vascular endothelial growth factor augments muscle blood flow and function in a rabbit model of chronic hindlimb ischemia. Journal of cardiovascular pharmacology 27, 91-98 (1996). 
Schwarz, E. R. et al. Evaluation of the effects of intramyocardial injection of DNA expressing vascular endothelial growth factor (VEGF) in a myocardial infarction model in the rat-angiogenesis and angioma formation. Journal of the American College of Cardiology 35, 1323-1330 (2000).

18 Yoshimura, K. et al. Cell-assisted lipotransfer for cosmetic breast augmentation: supportive use of adipose-derived stem/stromal cells. Aesthetic plastic surgery 32, 48-55; discussion 5647, doi:10.1007/s00266-007-9019-4 (2008).

19 Yoshimura, K. et al. Cell-assisted lipotransfer for facial lipoatrophy: efficacy of clinical use of adipose-derived stem cells. Dermatologic surgery : official publication for American Society for Dermatologic Surgery [et al.] 34, 1178-1185, doi:10.1111/j.1524-4725.2008.34256.x (2008).

20 Peltoniemi, H. H. et al. Stem cell enrichment does not warrant a higher graft survival in lipofilling of the breast: a prospective comparative study. Journal of plastic, reconstructive \& aesthetic surgery : JPRAS 66, 1494-1503, doi:10.1016/j.bjps.2013.06.002 (2013).

21 Rehman, J. et al. Secretion of angiogenic and antiapoptotic factors by human adipose stromal cells. Circulation 109, 1292-1298, doi:10.1161/01.cir.0000121425.42966.f1 (2004).

$22 \mathrm{Kim}, \mathrm{W}$. S. et al. Wound healing effect of adipose-derived stem cells: a critical role of secretory factors on human dermal fibroblasts. Journal of dermatological science 48, 15-24, doi:10.1016/j.jdermsci.2007.05.018 (2007).

23 Nakagami, H. et al. Novel autologous cell therapy in ischemic limb disease through growth factor secretion by cultured adipose tissue-derived stromal cells. Arteriosclerosis, thrombosis, and vascular biology 25, 2542-2547, doi:10.1161/01.ATV.0000190701.92007.6d (2005).

24 Kondo, K. et al. Implantation of adipose-derived regenerative cells enhances ischemiainduced angiogenesis. Arteriosclerosis, thrombosis, and vascular biology 29, 61-66, doi:10.1161/atvbaha.108.166496 (2009).

25 Matsuda, K. et al. Adipose-derived stem cells promote angiogenesis and tissue formation for in vivo tissue engineering. Tissue engineering. Part $A$ 19, 1327-1335, doi:10.1089/ten.TEA.2012.0391 (2013).

26 Kolle, S. F. et al. Enrichment of autologous fat grafts with ex-vivo expanded adipose tissuederived stem cells for graft survival: a randomised placebo-controlled trial. Lancet (London, England) 382, 1113-1120, doi:10.1016/s0140-6736(13)61410-5 (2013).

27 Sheikh, l. et al. Effect of electrical stimulation on arteriogenesis and angiogenesis after bilateral femoral artery excision in the rabbit hind-limb ischemia model. Vascular and endovascular surgery 39, 257-265, doi:10.1177/153857440503900307 (2005).

28 Kanno, S. et al. Establishment of a simple and practical procedure applicable to therapeutic angiogenesis. Circulation 99, 2682-2687 (1999).

29 Bai, H., Forrester, J. V. \& Zhao, M. DC electric stimulation upregulates angiogenic factors in endothelial cells through activation of VEGF receptors. Cytokine 55, 110-115, doi:10.1016/j.cyto.2011.03.003 (2011).

$30 \mathrm{Kim}, \mathrm{I}$. S. et al. Novel effect of biphasic electric current on in vitro osteogenesis and cytokine production in human mesenchymal stromal cells. Tissue engineering. Part A 15, 2411-2422, doi:10.1089/ten.tea.2008.0554 (2009).

31 Sebastian, A. et al. Acceleration of cutaneous healing by electrical stimulation: degenerate electrical waveform down-regulates inflammation, up-regulates angiogenesis and advances remodeling in temporal punch biopsies in a human volunteer study. Wound repair and regeneration : official publication of the Wound Healing Society [and] the European Tissue Repair Society 19, 693-708, doi:10.1111/j.1524-475X.2011.00736.x (2011).

32 Ud-Din, S. et al. Angiogenesis is induced and wound size is reduced by electrical stimulation in an acute wound healing model in human skin. PloS one 10, e0124502, doi:10.1371/journal.pone.0124502 (2015). 
Langelaan, M. L. et al. Advanced maturation by electrical stimulation: Differences in response between $\mathrm{C} 2 \mathrm{C} 12$ and primary muscle progenitor cells. Journal of tissue engineering and regenerative medicine 5, 529-539, doi:10.1002/term.345 (2011).

Reckhenrich, A. K. et al. Surgical sutures filled with adipose-derived stem cells promote wound healing. PloS one 9, e91169, doi:10.1371/journal.pone.0091169 (2014).

Moens, S., Goveia, J., Stapor, P. C., Cantelmo, A. R. \& Carmeliet, P. The multifaceted activity of VEGF in angiogenesis - Implications for therapy responses. Cytokine \& growth factor reviews 25, 473-482, doi:10.1016/j.cytogfr.2014.07.009 (2014).

Aplin, A. C., Fogel, E. \& Nicosia, R. F. MCP-1 promotes mural cell recruitment during angiogenesis in the aortic ring model. Angiogenesis 13, 219-226, doi:10.1007/s10456-0109179-8 (2010).

37 Stefansson, S. et al. Inhibition of angiogenesis in vivo by plasminogen activator inhibitor-1. The Journal of biological chemistry 276, 8135-8141, doi:10.1074/jbc.M007609200 (2001).

Tandon, N. et al. Alignment and elongation of human adipose-derived stem cells in response to direct-current electrical stimulation. Conference proceedings : ... Annual International Conference of the IEEE Engineering in Medicine and Biology Society. IEEE Engineering in Medicine and Biology Society. Annual Conference 2009, 6517-6521, doi:10.1109/iembs.2009.5333142 (2009).

39 Silva, E. A. \& Mooney, D. J. Effects of VEGF temporal and spatial presentation on angiogenesis. Biomaterials 31, 1235-1241, doi:10.1016/j.biomaterials.2009.10.052 (2010).

40 Rackauskas, G. et al. Subthreshold High-Frequency Electrical Field Stimulation Induces VEGF Expression in Cardiomyocytes. Cell transplantation 24, 1653-1659, doi:10.3727/096368914x682783 (2015).

$41 \mathrm{Kim}, \mathrm{I}$. S. et al. Biphasic electric current stimulates proliferation and induces VEGF production in osteoblasts. Biochimica et biophysica acta 1763, 907-916, doi:10.1016/j.bbamcr.2006.06.007 (2006).

42 Hang, J., Kong, L., Gu, J. W. \& Adair, T. H. VEGF gene expression is upregulated in electrically stimulated rat skeletal muscle. The American journal of physiology 269, H1827-1831 (1995).

43 McLean, N. A. \& Verge, V. M. Dynamic impact of brief electrical nerve stimulation on the neural immune axis-polarization of macrophages toward a pro-repair phenotype in demyelinated peripheral nerve. Glia 64, 1546-1561, doi:10.1002/glia.23021 (2016).

44 Zubkova, E. S. et al. Regulation of Adipose Tissue Stem Cells Angiogenic Potential by Tumor Necrosis Factor-Alpha. Journal of cellular biochemistry 117, 180-196, doi:10.1002/jcb.25263 (2016).

45 Lee, M. J. et al. Proteomic analysis of tumor necrosis factor-alpha-induced secretome of human adipose tissue-derived mesenchymal stem cells. Journal of proteome research $\mathbf{9}$, 1754-1762, doi:10.1021/pr900898n (2010).

46 Qin, Q. et al. Effect and mechanism of thrombospondin-1 on the angiogenesis potential in human endothelial progenitor cells: an in vitro study. PloS one 9, e88213, doi:10.1371/journal.pone.0088213 (2014).

47 Akahane, T., Akahane, M., Shah, A., Connor, C. M. \& Thorgeirsson, U. P. TIMP-1 inhibits microvascular endothelial cell migration by MMP-dependent and MMP-independent mechanisms. Experimental cell research 301, 158-167, doi:10.1016/j.yexcr.2004.08.002 (2004).

48 Carmeliet, P. Angiogenesis in life, disease and medicine. Nature 438, 932-936, doi:10.1038/nature04478 (2005).

49 Zhao, M., Bai, H., Wang, E., Forrester, J. V. \& McCaig, C. D. Electrical stimulation directly induces pre-angiogenic responses in vascular endothelial cells by signaling through VEGF receptors. Journal of cell science 117, 397-405, doi:10.1242/jcs.00868 (2004).

50 Hammerick, K. E., Longaker, M. T. \& Prinz, F. B. In vitro effects of direct current electric fields on adipose-derived stromal cells. Biochemical and biophysical research communications 397, 12-17, doi:10.1016/j.bbrc.2010.05.003 (2010). 
51 Hammerick, K. E., James, A. W., Huang, Z., Prinz, F. B. \& Longaker, M. T. Pulsed direct current electric fields enhance osteogenesis in adipose-derived stromal cells. Tissue engineering. Part A 16, 917-931, doi:10.1089/ten.TEA.2009.0267 (2010).

52 McCullen, S. D. et al. Application of low-frequency alternating current electric fields via interdigitated electrodes: effects on cellular viability, cytoplasmic calcium, and osteogenic differentiation of human adipose-derived stem cells. Tissue engineering. Part C, Methods 16, 1377-1386, doi:10.1089/ten.TEC.2009.0751 (2010).

53 Esfandiari, E. et al. The effect of high frequency electric field on enhancement of chondrogenesis in human adipose-derived stem cells. Iranian journal of basic medical sciences 17, 571-576 (2014).

54 Ceccarelli, G. et al. A comparative analysis of the in vitro effects of pulsed electromagnetic field treatment on osteogenic differentiation of two different mesenchymal cell lineages. BioResearch open access 2, 283-294, doi:10.1089/biores.2013.0016 (2013).

55 Razavi, S., Salimi, M., Shahbazi-Gahrouei, D., Karbasi, S. \& Kermani, S. Extremely lowfrequency electromagnetic field influences the survival and proliferation effect of human adipose derived stem cells. Advanced biomedical research 3, 25, doi:10.4103/22779175.124668 (2014).

56 Bodamyali, T., Kanczler, J. M., Simon, B., Blake, D. R. \& Stevens, C. R. Effect of faradic products on direct current-stimulated calvarial organ culture calcium levels. Biochemical and biophysical research communications 264, 657-661, doi:10.1006/bbrc.1999.1355 (1999).

57 Behrens, B. Physical agents theory and practice. 3rd edn, 262 (F.A. Davis Company, 2014).

58 Bussche, L. \& Van de Walle, G. R. Peripheral Blood-Derived Mesenchymal Stromal Cells Promote Angiogenesis via Paracrine Stimulation of Vascular Endothelial Growth Factor Secretion in the Equine Model. Stem cells translational medicine 3, 1514-1525, doi:10.5966/sctm.2014-0138 (2014).

59 Le Noble, F. A., Hekking, J. W., Van Straaten, H. W., Slaaf, D. W. \& Struyker Boudier, H. A. Angiotensin II stimulates angiogenesis in the chorio-allantoic membrane of the chick embryo. European journal of pharmacology 195, 305-306 (1991).

60 Guido, B. C. et al. Impact of kinesin Eg5 inhibition by 3,4-dihydropyrimidin-2(1H)-one derivatives on various breast cancer cell features. BMC cancer 15,283 , doi:10.1186/s12885015-1274-1 (2015). 


\section{CHAPTER 7}

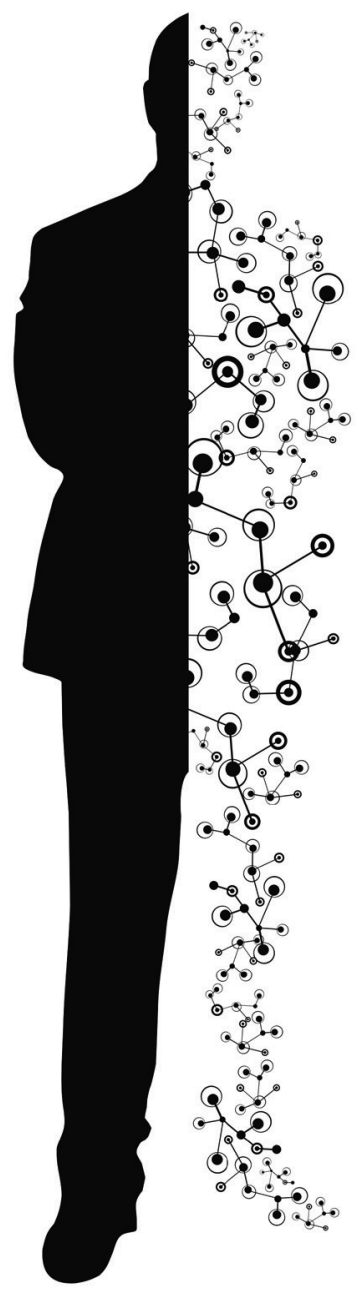




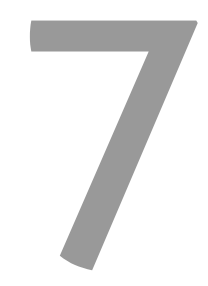

General discussion 


\section{General discussion}

With today's detailed anatomical knowledge and the sophistication of surgical tools and techniques, we have reached to some extent the border of the technical feasibility to optimize surgical outcome. Even with more advanced surgical techniques, the procedure will still evoke an inflammatory response in the operated area, which is beneficial at first, but negatively impacts the outcome if this inflammatory response persists. Surgical procedures such as AFT, keep relying heavily on the capability of the surrounding tissue to revascularize the graft through angiogenesis. For this reason cell-based therapeutics and modification hereof holds so much promise to keep on improving surgical procedures in the future since they modify these processes in order to optimize form and function. The aim of this thesis was therefore to investigate the effect of stem cells on inflammation and angiogenesis in different (extreme) models of neuro-injury and angiogenesis.

\section{Autologous Fat Transfer}

In chapter $\mathbf{2}$ we performed a systematic review and meta-analysis regarding AFT in facial reconstructive surgery. The extensive meta-analysis showed that AFT is a safe procedure with a high rate of patient (91\%) and surgeon (89\%) satisfaction. Complications occurred in $5 \%$ of procedures, of which when asymmetry and overcorrection were left out, $0.8 \%$ was a 'true' surgery related complication (1755 AFT procedures: 2 infections, 2 cases of fat necrosis and 10 hematomas). The wide variation in 'graft take' mentioned in the introduction was also visible in this study as retention varied between $40 \%$ to over $80 \%$ with a pooled effect estimate of $59 \%$ at one year in patients with congenital facial deformities. In HIVlipodystrophy patients this value ranged from $51 \%$ at one year, to $238 \%$, a major increase which could be due to the commonly reported adverse event lipohypertrophy after AFT in HIV-patients. These findings were similar in meta-analyses of AFT in other areas, such as breast reconstruction ${ }^{1}$ and imply that AFT is a safe and well received treatment, yet a significant part of the graft volume is lost after time which has led to an ongoing quest to improve retained graft volume. 
In chapter $\mathbf{3}$ we investigated whether patients with chronic neuropathic pain without apparent cause (such as a neuroma) and who were non responders to conventional treatment could benefit from AFT in the area around the affected nerve. No complications were registered, patient satisfaction was high (86\%) after more than one year follow-up and the level of pain scored on a VAS scale decreased from 7.4 pre-operative to 3.8 directly postoperative $(p<0.0001)$ and $4.3(p=0.0017)$ at long-term follow-up. This study proves that AFT can also be used to improve function, irrespective of form. Although we did not gather data on the mechanisms leading to this neuropathic pain-reducing effect of AFT, nor looked into the cell populations responsible for the effect, Vaienti et al speculated that both a mechanical effect ("cushioning" the affected nerve) and a biological effect that is mainly based on immunomodulation and angiogenesis by regenerative cells in the graft lead to a favorable environment for correct axonal repair. ${ }^{2}$ These regenerative cells may very well be ASC. Lin et al found that autologous ASC transplantation in rats with burn injuries attenuate local inflammation and central neuroinflammation and ameliorated autophagy and apoptosis in the spinal cord. ${ }^{3}$ For definitive clinical evidence on the use of AFT for neuropathic pain, prospective controlled studies with a larger study population and control/sham groups as well as mechanistic studies are warranted.

\section{Immunomodulation}

When a surgical incision is made, contact between platelets and the extracellular matrix induces the release of granules containing clotting factors and inflammatory cytokines. In response, neutrophils find their way to the injured site, followed by macrophages and leucocytes in an organized effort to clear foreign antigens and (cellular) debris. ${ }^{4}$ Chemotaxis of regenerative cells such as MSCs occurs through signaling by a variety of cytokines. Both resident and recruited MSCS are triggered by pro-inflammatory cytokines like TNF- $\alpha$, INF- $\gamma$ and IL-1 $\beta$ which in turn activate regulatory functions that lead to an inhibition of the recruitment, proliferation and activity of pro-inflammatory immune cells. ${ }^{5,6}$

In chapter 4 we administered a cocktail of minimally manipulated human bone-marrow derived MSCs, HSCs and other mononuclear cells called Neuro-Cells (NC) to both sham and $\mathrm{SCl}$-lesioned T-cell deficient rats. At day 4 the early immune response was investigated by quantifying serum levels of key inflammatory proteins. There was a significant increase in serum concentration of $\mathrm{IL}-1 \beta$ and TNF- $\alpha$ in SCl-lesioned versus sham-lesioned animals 
$(\mathrm{P}<0.05)$. However, this was not true for $\mathrm{NC}$-treated $\mathrm{SCl}$ animals, possibly indicating attenuation by the added stem cells. A similar effect, revealing decreased levels of IL-1 $\beta$ and TNF- $\alpha$, was found after intrathecal application of human MSCs in rats by Urdzikova et al. ${ }^{7}$ While in our study IL-6 was lower in the NC treated SCl-lesioned animals compared to their vehicle-treated counterparts both were still significantly higher as compared to the sham groups. In addition, immunohistochemical analyses were performed of the sampled spinal cords at day 4 focusing on the marker CD68, which is found on microglia and macrophages. Interestingly, a significant increase of $\mathrm{CD}^{+} 8^{+}$cells was found caudal to the lesion site in NCtreated $\mathrm{SCl}$-lesioned animals versus vehicle-treated $\mathrm{SCl}$-lesioned animals, with a moderate increase at the lesion site itself and no difference rostral to the lesion site. Explanations as to why there were more microglia/macrophages in the NC treated group include a more profound damage-response reaction to the injection of rather viscous Neuro-Cells and detection of possibly human and rat macrophages since the anti-CD68 antibody was both specific for rat as for human CD68. With more $\mathrm{CD}^{+} 8^{+}$cells present in $\mathrm{NC}$ treated $\mathrm{SCl}$ rats, knowing they are a prime source for IL-1 $\beta$ and TNF- $\alpha$, it is even more surprising that the levels of inflammatory cytokines in the serum are lower and potentially highlights the immunomodulatory effect of MSC and HSC. The subsequent steps are therefore to characterize the function M1 or M2 of the recruited cells over time. Is a macrophage beneficial or detrimental after surgery?

We found in this model of $\mathrm{SCl}$ that the administration of $\mathrm{NC}$ not only reduced inflammation but also improved function. This functional outcome is paramount since only improved function means an improvement in quality of life for the patient. The basic lesson in this experiment was that even central nervous function can be protected/regenerated by the administration of local NC. We have not conducted detailed mechanistic analysis but the available data point towards anti-inflammatory effects which in the long run result in better cell survival and function. ${ }^{8}$

\section{Angiogenesis}

Tissue perfusion is key to the success of every surgical procedure. This aspect is easily visualized in free-flap reconstruction in plastic surgery. Blood flow is re-established through the flap after surgical anastomosis of the blood vessels. However, if the blood flow is insufficient (usually in the most distal part of the flap), ischemic necrosis occurs. In non- 
vascularized tissue transfer, such as AFT, early revascularization is of paramount importance. Without it, all cells that are out of diffusion range undergo the same process of ischemic necrosis. Fat grafts are revascularized by formation of a new vascular network through the process of angiogenesis. Angiogenesis starts in the remodeling phase of healing around 3 days after the initial tissue trauma, stimulated by factors in the microenvironment such as hypoxia and by angiogenic factors produced in the prior days by surrounding cells such as macrophages, (mesenchymal) stem cells, fibroblasts and endothelial cells. ${ }^{9}$ However, in fat grafts the majority of adipocytes perish within the first 24 hours, well before the onset of angiogenesis. ${ }^{10,11}$ Since stem cells tolerate hypoxia for several days and are a prime source of pro-angiogenic factors, many studies have investigated the effect on angiogenesis and tissue survival by adding all kinds of stem cells to the affected site in different models of angiogenesis. $^{12-15}$

In chapter 5 different dosages of human unfractionated bone marrow-derived cells (containing mesenchymal and hematopoietic stem cells) were intramuscularly and intravenously administered to T-cell deficient rats who had undergone unilateral ligation of the arteria iliaca externa 7 days prior. At day 35 differences in vascular density between animals who had received cell preparations and the control group (which received only carrier, $\mathrm{NaCl}$ ) were compared using Mean Gray Values (MGV) established through Digital Subtraction Angiography (DSA). Three different dosages were standardized by the number of $\mathrm{CD}^{+} 4^{+}$cells: low, medium and high. Whereas the low and medium group showed a statistically significant increase in vascular density, the high concentration group did not. There was no correlation between the total number of $\mathrm{CD}_{3} 4^{+}$cells and the MGV in this study so we were unable to make a statement on dose-dependency. This is in line with other studies in both animal models and clinical trials, in which the total cell number, the site of administration and the regime (single vs multiple injections and IV vs IM administration) appeared not to affect efficacy. ${ }^{16}$ However, the high dose group actually led to the death of $2 / 5$ animals during administration (most likely due to hyper viscosity of the cell preparation) highlighting the importance of a refined and standardized application of cell preparations in order to avoid such complications.

The two different cell preparations in chapter 4 and 5 highlight the general problem of cell processing and conditioning. These cell preparations were unfractionated and processed 
with Neuroplast's Rapid Interventional Stem Cells Platform, which is a GMP-compliant closed system that generates NC within 6 hours of aspirating crude bone marrow. It would be interesting to find out which subpopulation of the NC has the highest impact on vascular density. However, this mandates isolation and at least some form of manipulation or labeling of cells in the lab, before administering the cells to the patient. This approach faces huge regulatory hurdles in Europe and the US regarding the safety of ex vivo manipulated cells and the agents/chemicals that have been in contact with the cells and thus may be transferred into the body. ${ }^{17}$

The same hurdle is described in the scientific literature regarding AFT. In an effort to enrich fat grafts with regenerative cells, Cytori therapeutics has developed the Celution ${ }^{\circledR}$ system that isolates the stromal vascular fraction (containing ASC) from the patient's own fat in the same procedure. ${ }^{18}$ However, regulatory issues still persist since the system uses collagenase to digest the fat. In addition, the system itself is relatively costly to use and extends surgery and anesthesia time by at least 90 minutes, meaning higher risks, costs and less patients operated per day. ${ }^{19}$

The approach of manipulating or expanding cell populations ex vivo is thus not a simple solution to the underlying problem. Angiogenesis is mandatory for the survival of transplanted cells in AFT. We envisioned therefore manipulation/conditioning of the patient's own cell in vivo by a method that is neither invasive nor harmful to the patient. Instead of administering exogenous cells whose primary function is to secrete proangiogenic/immunomodulatory cytokines and growth factors, an alternative approach would be to use an external stimulus to influence native cells to upregulate their own secretion instead. Electrical stimulation is one of those stimuli, is rather inexpensive, noninvasive, safe, and clinically approved. In a rabbit ischemic hind limb model it stimulated both arteriogenesis and angiogenesis ${ }^{20}$, but a potential pro-angiogenic effect on ASC had never been investigated. Therefore, we performed a proof-of-principle study to determine whether ES of ASC can stimulate in vitro and in vivo parameters of angiogenesis in chapter 6.

Cultured human ASC were electrically stimulated for 72 hours after which the medium of stimulated (ES) and non-stimulated (control) ASC was analyzed for angiogenesisrelated proteins and then tested for a functional effect in an in vivo model of angiogenesis. Two pro-angiogenic proteins (VEGF and MCP-1) were significantly increased while the antiangiogenic growth factor Serpin E1/PAI-1 was significantly decreased. Despite the fact that 
the levels of two anti-angiogenic growth factors TSP-1 and TIMP-1 were also increased, medium of ES-treated ASC significantly increased vessel density, total vessel network length and branching points in a chorio-allantoic membrane assay. So even though two inhibitors of angiogenesis were upregulated, we found an overall proangiogenic effect in an in vivo model, hinting at the possibility that ES might leave feedback mechanisms in place that prevent unrestricted angiogenesis.

Furthermore, it is interesting that secretion of MCP-1 was increased because monocyte chemoattractant protein-1 recruits monocytes to the site of injury. At first glance this might imply a pro-inflammatory effect of ES, however other studies ${ }^{21,22}$ have shown that treatment of ASC with the pro-inflammatory stimulus TNF- $\alpha$ also increased levels of SerpinE1/PAI-1 whiles ES caused the opposite to happen in our study, suggesting that ES is not the equivalent of a general pro-inflammatory stimulus. On the other hand, macrophages play an important role in regeneration. They comprise the majority of immune cells present in the SVF of adipose tissue, ranging from $5 \%$ of the SVF cells in lean mice and humans to about $50 \%$ in obese individuals $s^{23,24}$ and have been shown to produce a great number of pro- (and anti-) angiogenic factors, such as VEGF-A, FGF-2 and PDGF-BB. ${ }^{25}$ Navarro and colleagues even report a greater influence of macrophages on angiogenesis than that of ASC. ${ }^{26}$ Phipps et al found a stimulatory effect of added M2 macrophages on the volume retention of a fat graft in mice. ${ }^{27}$

In addition, the timeframe, in which significant elevation of pro-angiogenic factors can be reached, has important clinical applications. To put our findings into perspective: most adipocytes in the inner layers of the fat graft die within 24 hours of ischemia. ${ }^{10,11}$ Whereas in our study, involving ASC, ES induced a significantly higher concentration of VEGF after 3 days, in BM-MSC, Kim et al found a small but statistically significant increase of VEGF after electrical stimulation of BM-MSC after 1 day and a more profound effect after 2 days. ${ }^{28}$ This finding implies that it could be beneficial to already start treatment of the recipient site before AFT, in order to capitalize on a better vascular network and already activated angiogenesis. Another possibility, even in conjunction with the latter, would be to pretreat the donor site in order to already prime and activate the resident (stem) cells that soon after will be transferred. Subsequent studies have to determine this. 


\section{References}

1 Krastev, T. K., Alshaikh, G. A. H., Hommes, J., Piatkowski, A. \& van der Hulst, R. Efficacy of autologous fat transfer for the correction of contour deformities in the breast: A systematic review and meta-analysis. Journal of plastic, reconstructive \& aesthetic surgery : JPRAS 71, 1392-1409, doi:10.1016/j.bjps.2018.05.021 (2018).

2 Vaienti, L., Merle, M., Battiston, B., Villani, F. \& Gazzola, R. Perineural fat grafting in the treatment of painful end-neuromas of the upper limb: a pilot study. The Journal of hand surgery, European volume 38, 36-42, doi:10.1177/1753193412441122 (2013).

Lin, C. H. et al. Autologous Adipose-Derived Stem Cells Reduce Burn-Induced Neuropathic Pain in a Rat Model. International journal of molecular sciences 19, doi:10.3390/ijms19010034 (2017).

4 Barrientos, S., Stojadinovic, O., Golinko, M. S., Brem, H. \& Tomic-Canic, M. Growth factors and cytokines in wound healing. Wound repair and regeneration : official publication of the Wound Healing Society [and] the European Tissue Repair Society 16, 585-601, doi:10.1111/j.1524-475X.2008.00410.x (2008).

5 Hemeda, H. et al. Interferon-gamma and tumor necrosis factor-alpha differentially affect cytokine expression and migration properties of mesenchymal stem cells. Stem cells and development 19, 693-706, doi:10.1089/scd.2009.0365 (2010).

6 Ren, G. et al. Mesenchymal stem cell-mediated immunosuppression occurs via concerted action of chemokines and nitric oxide. Cell stem cell 2, 141-150, doi:10.1016/j.stem.2007.11.014 (2008).

7 Urdzikova, L. M. et al. Human mesenchymal stem cells modulate inflammatory cytokines after spinal cord injury in rat. International journal of molecular sciences 15, 11275-11293, doi:10.3390/ijms150711275 (2014).

8 Zhang, N., Yin, Y., Xu, S. J., Wu, Y. P. \& Chen, W. S. Inflammation \& apoptosis in spinal cord injury. The Indian journal of medical research 135, 287-296 (2012).

9 Olczyk, P., Mencner, L. \& Komosinska-Vassev, K. The role of the extracellular matrix components in cutaneous wound healing. BioMed research international 2014, 747584, doi:10.1155/2014/747584 (2014).

10 Suga, H. et al. Adipose tissue remodeling under ischemia: death of adipocytes and activation of stem/progenitor cells. Plastic and reconstructive surgery 126, 1911-1923, doi:10.1097/PRS.0b013e3181f4468b (2010).

11 Eto, H. et al. The fate of adipocytes after nonvascularized fat grafting: evidence of early death and replacement of adipocytes. Plastic and reconstructive surgery 129, 1081-1092, doi:10.1097/PRS.0b013e31824a2b19 (2012).

12 Al-Khaldi, A., Al-Sabti, H., Galipeau, J. \& Lachapelle, K. Therapeutic angiogenesis using autologous bone marrow stromal cells: improved blood flow in a chronic limb ischemia model. The Annals of thoracic surgery 75, 204-209, doi:10.1016/s0003-4975(02)04291-1 (2003).

13 Moon, M. H. et al. Human adipose tissue-derived mesenchymal stem cells improve postnatal neovascularization in a mouse model of hindlimb ischemia. Cellular physiology and biochemistry : international journal of experimental cellular physiology, biochemistry, and pharmacology 17, 279-290, doi:10.1159/000094140 (2006).

14 Lu, F. et al. Improvement of the survival of human autologous fat transplantation by using VEGF-transfected adipose-derived stem cells. Plastic and reconstructive surgery 124, 14371446, doi:10.1097/PRS.0b013e3181babbb6 (2009).

15 Piccinno, M. S. et al. Adipose stromal/stem cells assist fat transplantation reducing necrosis and increasing graft performance. Apoptosis : an international journal on programmed cell death 18, 1274-1289, doi:10.1007/s10495-013-0878-7 (2013). 
Liew, A. \& O'Brien, T. Therapeutic potential for mesenchymal stem cell transplantation in critical limb ischemia. Stem cell research \& therapy 3, 28, doi:10.1186/scrt119 (2012).

17 Kim, Y. J. \& Jeong, J. H. Clinical application of adipose stem cells in plastic surgery. Journal of Korean medical science 29, 462-467, doi:10.3346/jkms.2014.29.4.462 (2014).

Yoshimura, K. et al. Cell-assisted lipotransfer for facial lipoatrophy: efficacy of clinical use of adipose-derived stem cells. Dermatologic surgery : official publication for American Society for Dermatologic Surgery [et al.] 34, 1178-1185, doi:10.1111/j.1524-4725.2008.34256.x (2008).

19 van Dongen, J. A. et al. Comparison of intraoperative procedures for isolation of clinical grade stromal vascular fraction for regenerative purposes: a systematic review. Journal of tissue engineering and regenerative medicine 12, e261-e274, doi:10.1002/term.2407 (2018).

20 Sheikh, l. et al. Effect of electrical stimulation on arteriogenesis and angiogenesis after bilateral femoral artery excision in the rabbit hind-limb ischemia model. Vascular and endovascular surgery 39, 257-265, doi:10.1177/153857440503900307 (2005).

21 Zubkova, E. S. et al. Regulation of Adipose Tissue Stem Cells Angiogenic Potential by Tumor Necrosis Factor-Alpha. Journal of cellular biochemistry 117, 180-196, doi:10.1002/jcb.25263 (2016).

22 Lee, M. J. et al. Proteomic analysis of tumor necrosis factor-alpha-induced secretome of human adipose tissue-derived mesenchymal stem cells. Journal of proteome research $\mathbf{9}$, 1754-1762, doi:10.1021/pr900898n (2010).

23 Weisberg, S. P. et al. Obesity is associated with macrophage accumulation in adipose tissue. The Journal of clinical investigation 112, 1796-1808, doi:10.1172/jci19246 (2003).

24 Ortega Martinez de Victoria, E. et al. Macrophage content in subcutaneous adipose tissue: associations with adiposity, age, inflammatory markers, and whole-body insulin action in healthy Pima Indians. Diabetes 58, 385-393, doi:10.2337/db08-0536 (2009).

25 Corliss, B. A., Azimi, M. S., Munson, J. M., Peirce, S. M. \& Murfee, W. L. Macrophages: An Inflammatory Link Between Angiogenesis and Lymphangiogenesis. Microcirculation (New York, N.Y. : 1994) 23, 95-121, doi:10.1111/micc.12259 (2016).

26 Navarro, A., Marin, S., Riol, N., Carbonell-Uberos, F. \& Minana, M. D. Human adipose tissueresident monocytes exhibit an endothelial-like phenotype and display angiogenic properties. Stem cell research \& therapy 5, 50, doi:10.1186/scrt438 (2014).

27 Phipps, K. D. et al. Alternatively activated M2 macrophages improve autologous Fat Graft survival in a mouse model through induction of angiogenesis. Plastic and reconstructive surgery 135, 140-149, doi:10.1097/prs.0000000000000793 (2015).

$28 \mathrm{Kim}, \mathrm{l}$. S. et al. Novel effect of biphasic electric current on in vitro osteogenesis and cytokine production in human mesenchymal stromal cells. Tissue engineering. Part A 15, 2411-2422, doi:10.1089/ten.tea.2008.0554 (2009). 


\section{CHAPTER 8}

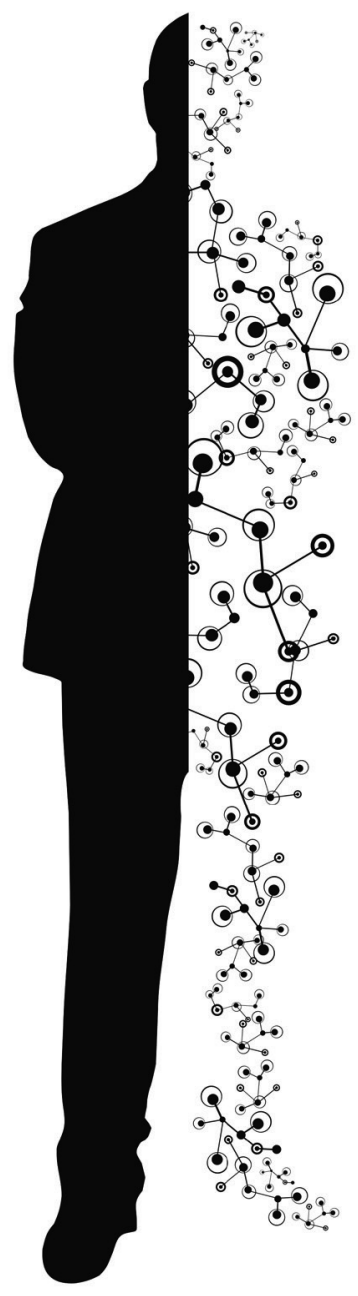


Summary 


\section{Summary}

\section{Chapter 1. General introduction}

In plastic and reconstructive surgery many surgical procedures have been developed to restore form and/or function. Autologous fat transfer (AFT), in which adipose tissue is relocated from one place to the other is a recent example that is safe and versatile. An important aspect in the final outcome for the patient is the state of the local microenvironment in the regenerating tissue. However great the surgeon or surgical technique may be, they have no direct way of modifying the local microenvironment to promote healing. Great potential herein lies with the use of (adult) stem cells. This thesis investigated the effect of stem cells on two important cellular processes, occurring after (surgical) tissue trauma: inflammation and angiogenesis. Ideally we would have a technique to directly "activate" tissue resident stem cells and guide them towards promoting angiogenesis or modulating inflammation. A technique called electrical stimulation is promising in this area.

Chapter 2. Efficacy and safety of autologous fat transfer in facial reconstructive surgery: a systematic review and meta-analysis.

In chapter 2, we focused specifically on AFT through analysis of the efficacy and safety of performing AFT in plastic surgery. We compiled the available clinical data from 52 relevant studies consisting of 1568 unique patients in a systematic review and meta-analysis. It showed a very high overall satisfaction rate of $91.1 \%$ in patients, together with a $88.6 \%$ satisfaction rate in surgeons. In facial reconstructive surgery the mean number of sessions to achieve the desired end result was 1.5 , with a retention rate of $50-60 \%$ of the grafted material. Less than $5 \%$ of the procedures resulted in minor complications, deeming it a safe procedure. 
Chapter 3. Autologous fat transfer as a treatment for peripheral neuropathic pain without apparent cause.

In chapter 3, we tested in a clinical trial whether patients in a chronic neuropathic pain setting without a cause such as a neuroma, and who had exhausted all other treatment options, would benefit from AFT with respect to their pain perception. Fourteen patients received AFT in the area of the affected nerve. Outcome parameters included patient satisfaction, pain scored on a visual analogue scale (VAS) and quality of sleep. Patient satisfaction was $93 \%$ at the follow-up after two weeks and $86 \%$ after a follow-up of more than one year. The mean VAS score decreased significantly from 7.4 before surgery to 3.8 directly after AFT and 4.3 at long-term follow-up visit. The quality of sleep improved in $50 \%$ of the patients, whereas the remainder indicated no difference. No complications were registered. This study shows that AFT can be a suitable option for patients suffering from debilitating neuropathic pain that is refractory to other treatments. A potential mechanism of action lies in the regenerative capabilities of cells within the transplant.

\section{Chapter 4. Standardized human bone marrow-derived stem cells infusion improves} survival and recovery in a rat model of spinal cord injury.

In chapter 4, we set out to investigate the immunomodulatory properties of a refined bonemarrow derived preparation of mesenchymal and hematopoietic stem cells in an acute model of spinal cord injury. Our focus lay towards researching the anti-inflammatory properties and the effect on nerve regeneration in the context of improved functional outcome. To this end T-cell deficient rats, in which a spinal cord lesion (SCl) was induced by balloon-compression of the spinal cord, received an intrathecal injection of bone marrowderived stem cells (called Neuro-Cells; NC) the day after SCl. During the first 5 weeks after this intervention, NC significantly improved locomotor recovery and induced less injuryassociated adverse events compared to the vehicle-treated control group. Histological analyses showed that NC reduced astrogliosis and apoptosis primarily in the first days after administration. Proteomic studies of the sampled spinal cords at the study endpoint (56 days) pointed to the release of paracrine factors and identified proteins involved in regenerative processes. 
Chapter 5. Efficacy of different doses of human adult bone marrow stem cell transplantation on angiogenesis in a rat model with hind limb ischemia.

In chapter 5, we surgically ligated the external iliac artery of T-cell deficient rats to develop a hind limb ischemia model. The surgical ligation of arterial blood vessels lead to an acuteonset moderate to severe traumatic ischemia in the affected hind limbs. After 7 days a baseline digital subtraction angiography (DSA) was made. Then, different doses of human bone marrow-derived stem cells, or vehicle alone were administered intramuscularly and intra-arterially. At day 35, DSA was repeated and images were compared between the groups at the different time points to directly visualize the angiogenic effects of the administered stem cells. Comparison of DSA Mean Gray Values at day 35 versus day 7 showed a significant increase in the low and medium dose groups, pointing towards improved vascularization, whereas the vehicle group displayed a natural decrease. Surprisingly, the high dose stem cell group also had a decreased Mean Gray Value at day 35 and more tissue damage, possibly due to hyperviscosity. A dose response could not be detected.

Chapter 6. Electrical stimulation promotes the angiogenic potential of adipose-derived stem cells.

In chapter 6, we electrically stimulated adipose tissue-derived stem cells (ASC) in vitro to determine the effects of electrical stimulation (ES) on the angiogenic potential of ASC. Cultured human ASC were electrically stimulated for 72 hours after which the medium of stimulated (ES) and non-stimulated (control) ASC was analyzed for angiogenesis-related proteins by protein array and ELISA. Nine angiogenesis-related proteins were detected in the medium of electrically stimulated ASC. The pro-angiogenic proteins VEGF and MCP-1 were significantly increased following ES compared to controls, while the anti-angiogenic factor Serpin E1/PAI-1 was significantly decreased. Despite increased levels of anti-angiogenic TSP1 and TIMP-1, medium of ES-treated ASC significantly increased vessel density, total vessel network length and branching points in a functional in vivo model of angiogenesis, called a chorio-allantoic membrane assay. This proof-of-concept study showed that ES increased the angiogenic potential of ASC both in vitro and in vivo and lays the foundation for further translational studies. 


\section{Chapter 7. General discussion}

The general discussion reflects on the findings of the individual studies included in this thesis, starting with autologous fat transfer. Next chapters 4 through 6 were discussed in light of the topics immunomodulation and angiogenesis. Gaps in current knowledge are identified and proposals for future studies are provided. 


\section{CHAPTER 9}

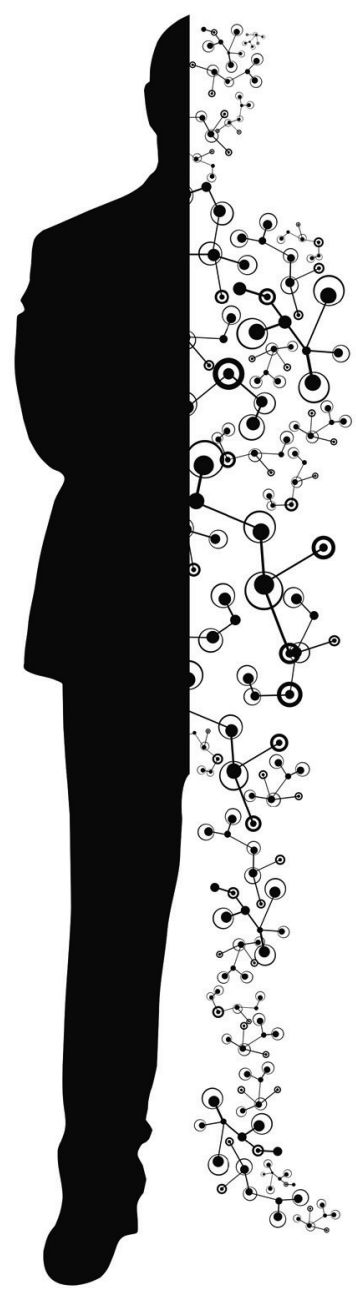




\section{Summary in dutch}

Nederlandse samenvatting 


\section{Summary in Dutch - Nederlandse samenvatting}

\section{Hoofdstuk 1. Algemene introductie}

In plastische en reconstructieve chirurgie zijn een groot aantal chirurgische procedures ontwikkeld om vorm en/of functie te herstellen. Autologe vet transfer (AFT), waarbij vet van de ene locatie naar de andere wordt gebracht is een recent voorbeeld dat zowel veilig als veelzijdig is. Een belangrijk aspect voor het uiteindelijke resultaat is de status van het lokale micromilieu in het regenererende weefsel. Hoe fantastisch de chirurg of de chirurgische techniek ook is, zij hebben geen directe mogelijkheid om het lokale micromilieu zodanig te beïnvloeden dat het de genezing bevordert. Het inzetten van stamcellen vertoont op dit vlak grote potentie. Deze thesis onderzocht het effect van stamcellen op twee belangrijke cellulaire processen die optreden na (chirurgisch) trauma: inflammatie en angiogenese. Idealiter zouden we een techniek wensen om direct de reeds aanwezige stamcellen in het weefsel te "activeren" en ze te stimuleren om angiogenese te bevorderen of inflammatie te moduleren. Een techniek genaamd elektrische stimulatie is veelbelovend voor deze indicatie.

Hoofdstuk 2. Effectiviteit en veiligheid van autologe vet transfer in faciale plastische chirurgie: een systematische review en meta-analyse.

In hoofdstuk 2 focusten we specifiek op AFT door een grondige analyse van de effectiviteit en veiligheid van AFT in plastische chirurgie. We compileerden de beschikbare klinische data van 52 relevante studies die in totaal bestonden uit 1568 unieke patiënten in een systematische review en meta-analyse. Het tevredenheidsniveau bleek algemeen erg hoog te zijn met $91.1 \%$ in de patiëntengroep en $88.6 \%$ bij de chirurgen. Het gemiddeld aantal sessies om het gewenste eindresultaat te bereiken was 1.5, met een retentiepercentage van $50-60 \%$ van het ingebrachte vetweefsel. Lichte complicaties traden op in minder dan $5 \%$ van de gevallen, waaruit geconcludeerd werd dat AFT in het gelaat een veilige ingreep is.

Hoofdstuk 3. Autologe vet transfer als een behandeling voor perifere neuropathische pijn zonder duidelijke oorzaak.

In hoofdstuk 3 onderzochten we in een klinische trial of patiënten met chronische neuropathische pijn zonder oorzaak zoals een neuroom, en die reeds alle andere 
mogelijkheden voor behandeling hadden geprobeerd, effect zouden hebben van AFT op het verminderen van de pijn. Veertien patiënten ontvingen AFT in het gebied van de aangedane zenuw. Tot de uitkomstparameters behoorden patiënttevredenheid, pijn gescoord op een visuele analoge schaal (VAS) en de kwaliteit van slaap. De patiënttevredenheid bedroeg 93\% bij de controle na 2 weken en $86 \%$ na een follow-up van meer dan één jaar. De gemiddelde VAS score daalde significant van 7.4 voor de ingreep tot 3.8 direct na AFT en 4.3 bij de controle op lange termijn. De kwaliteit van slaap verbeterde in $50 \%$ van de patiënten, waarbij de rest aangaf geen verschil te merken. Er werden geen complicaties geregistreerd. Deze studie toont dat AFT een mogelijke optie kan zijn in de behandeling van patiënten die lijden aan therapieresistente neuropathische pijn. Een potentieel werkingsmechanisme hiervan kan liggen in de regeneratieve eigenschappen van cellen in het vettransplantaat.

Hoofdstuk 4. Infusie met gestandaardiseerde uit beenmerg verkregen stamcellen verbetert de overleving en het herstel in een ratmodel van dwarslaesie.

In hoofdstuk 4 hebben we de immunomodulatoire eigenschappen van een geraffineerd preparaat met mesenchymale en hematopoïetische stamcellen uit beenmerg onderzocht in een acuut model van dwarslaesie. Onze focus lag bij het onderzoeken van de antiinflammatoire eigenschappen en het effect op zenuwregeneratie in de context van een verbeterde functionele uitkomst. Hiervoor kregen T-cel deficiënte ratten, bij wie een dwarslaesie was aangebracht door middel van balloncompressie, een intrathecale injectie met uit beenmerg verkregen stamcellen (genaamd Neuro-Cells; NC) de dag na de dwarslaesie. Gedurende de eerste 5 weken na de interventie verbeterden NC het motorische herstel significant en veroorzaakten zij minder trauma gerelateerde complicaties in vergelijking met de controle groep die behandeld was met alleen oplosvloeistof. Histologische analyses toonden aan dat NC astrogliose en apoptose vooral in de eerste dagen na toediening verminderden. Proteomisch onderzoek van het verzamelde ruggenmerg aan het einde van de studie (na 56 dagen) wees op vrijgelaten paracriene factoren en identificeerde eiwitten betrokken bij regeneratieve processen. 
Hoofdstuk 5. Effectiviteit van de transplantatie van verschillende doseringen van humane uit beenmerg verkregen stamcellen op angiogenese in een ratmodel met ischemie van de achterpoot.

In hoofdstuk 5 werd bij T-cel deficiënte ratten operatief de a. iliaca externa geligeerd om een diermodel met ischemie van de achterpoot te ontwikkelen. De chirurgische ligatie van arteriële bloedvaten leidde tot de acute ontwikkeling van middelmatige tot ernstige traumatische ischemie van de betrokken achterpoten. Na 7 dagen werd er een baseline digitale subtractie angiografie (DSA) gemaakt. Daarna werden er verschillende doseringen van humane uit beenmerg verkregen stamcellen, of alleen oplosvloeistof intramusculair en intra-arterieel toegediend. Op dag 35 werd de DSA herhaald en werden afbeeldingen vergeleken tussen de groepen op de verschillende tijdpunten om direct de angiogene effecten van de toegediende stamcellen te visualiseren. Vergelijking van de DSA Mean Gray Values op dag 35 versus dag 7 toonde een significante stijging in de lage en medium dosis groep, wijzende op verbeterde vascularisatie, terwijl de groep met alleen oplosvloeistof een natuurlijke daling liet zien. Verassend was dat de groep met een hoge dosis stamcellen ook een verminderde Mean Gray Value had op dag 35 met meer weefselschade, mogelijk door hyperviscositeit van het toegediende product. Een dosis-responscurve kon niet worden gedetecteerd.

Hoofdstuk 6. Elektrische stimulatie bevordert de angiogene potentie van stamcellen uit vetweefsel.

In hoofdstuk 6 hebben we stamcellen uit vetweefsel (adipose tissue-derived stem cells; ASC) in vitro gestimuleerd met elektrische stroom (ES) om het effect hiervan op de angiogene potentie van ASC te testen. Gekweekte humane ASC werden 72 uur elektrisch gestimuleerd waarna het kweekmedium van gestimuleerde en niet-gestimuleerde ASC werd onderzocht op angiogenese-gerelateerde eiwitten middels protein array en ELISA. Negen angiogenesegerelateerde eiwitten werden gedetecteerd in het medium van elektrisch gestimuleerde ASC. De proangiogene factoren VEGF en MCP-1 waren significant verhoogd na ES in vergelijking met de controlegroep, terwijl de anti-angiogene factor Serpin E1/PAI-1 significant was gedaald. Ondanks verhoogde niveaus van de anti-angiogene factoren TSP-1 en TIMP-1, vergrootte medium van gestimuleerde ASC significant de vaatdichtheid, totale 
lengte van het vaatnetwerk en het aantal vaatvertakkingen in een functioneel in vivo model van angiogenese, genaamd een chorioallantoïsch membraan onderzoek. Deze proof-ofconcept studie toonde aan dat ES de angiogene potentie van ASC zowel in vitro als in vivo bevordert en legt hiermee het fundament voor toekomstige translationele studies.

\section{Hoofdstuk 7. Algemene discussie}

In de algemene discussie worden de bevindingen van de individuele studies in deze thesis besproken, beginnend met autologe vet transfer. Daarna worden hoofdstukken 4 tot en met 6 besproken in het kader van de onderwerpen immunomodulatie en angiogenese. Hiaten in de huidige kennis worden geïdentificeerd en er worden voorstellen voor toekomstige studies gegeven. 


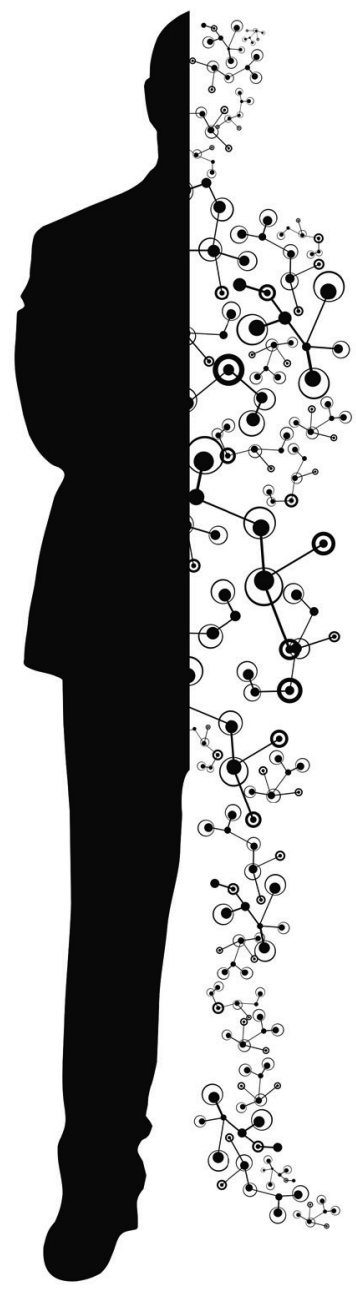




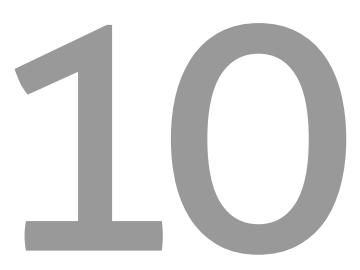

Valorization 


\section{Valorization}

Stem cell technology is thought by many medical professionals in the field to bring about the next era in medicine. Countless research groups are working towards harnessing the body's own regenerative capabilities in endogenous or isolated cells, and tailoring the therapy to a specific disease, condition, request or person. At the moment we still rely largely on medication to treat diseases, but businesswise as well as socially this is a horrible model, according to Dr. Susan Solomon in her 2012 TED talk: "Currently, developing a drug takes an average of 13 years, costs $\$ 4$ billion, and has a 99\% failure rate.", and: “The way we've been developing drugs is essentially like going into a shoe store and no one asking what size you are ... They just say, 'Well, you have feet. Here are shoes.'” This thesis was meant to explore new approaches to improve outcome in a variety of clinical conditions involving stem cells. This chapter focuses on discussing the societal and economic impact, as well as future implementation possibilities of the findings.

In Chapter 4, an animal study was carried out to assess the efficacy and safety of Neuro-cells, a standardized GMP-compliant and minimally manipulated product containing autologous stem cells from a patient's own bone marrow (derived from initial experiments in Chapter 3 ), on spinal cord injury. The prevalence of spinal cord injury $(\mathrm{SCl})$ worldwide is estimated at 2.5 million cases and the financial burden per case is calculated to be between 200,000 260,000 Euros/year, aside from the tremendous impact on the quality of life for the patient and surrounding people. ${ }^{1}$ The preclinical work in this chapter was part of a data set that has been used by the company Neuroplast to obtain an Orphan Drug Designation from the European Medicines Agency, allowing a fast track procedure for clinical trials. Currently phase I/II trials are being carried out. Neuroplast has raised $€ 4$ million in 2020 to proceed with a phase II/III study in 2021 and, if successful, obtain conditional market approval for treatment of patients suffering from spinal cord injury.

The proof-of-principle study in chapter 6, involving electrical stimulation (ES) of ASC in order to enhance their angiogenic properties, can pave the way for a non-invasive, painless procedure for the patient to optimize the results of AFT. Several subsequent steps can be taken based on the information that was obtained. These steps include a proof of principle in animals in which the effect of ES on AFT is investigated with a focus towards volume 
retention, graft composition and immunomodulation. Multiple groups can be included to determine whether it could be beneficial to pre-operatively treat the donor site ("priming the graft"), or the acceptor site ("pretreating the soil"). In addition, the effect of altering key stimulus parameters such as wave form, voltage, pulse duration and frequency needs to be studied to assess which ES protocol provides the best response. A basic problem however, may be the size of the graft when using small animals as this is not representative for humans. Graft survival due to plasmatic diffusion plays a bigger role and penetration of ES into the graft could be drastically different. Therefore, a choice has to be made to either go for a big animal model where also the penetration of the ES into the tissue can be assessed, or go straight into a clinical trial. ES devices for use in humans have been on the market for years. However, the safety of applying ES after breast cancer has to be established, especially when the therapy is aimed at stimulating angiogenesis. Today, on average 3-4 sessions of AFT are needed for a complete breast reconstruction after mastectomy. Every AFT is an operation with inherent risks, costs and recovery period. The reduction of the number of surgical procedures may therefore prove a valuable goal both financially and for the patient's wellbeing and safety.

This thesis further identified AFT as a potential treatment for neuropathic pain in Chapter 3. Neuropathic pain has a major influence on patients' lives and the people around them, not to mention the economic burden for society when debilitating pain leads to chronic unemployment. Our clinical proof of principle study provides the basis for a subsequent clinical study in which the effects of AFT are analyzed more in depth. The effect of adding ES to the treatment protocol is another avenue that is of interest and could be investigated in a randomized, controlled clinical trial. Non-responders to established treatments of neuropathic pain could be ideal patients for this clinical trial.

\section{References}

1. DeVivo MJ (1997) Causes and costs of spinal cord injury in the United States. Spinal Cord 35: 809813 


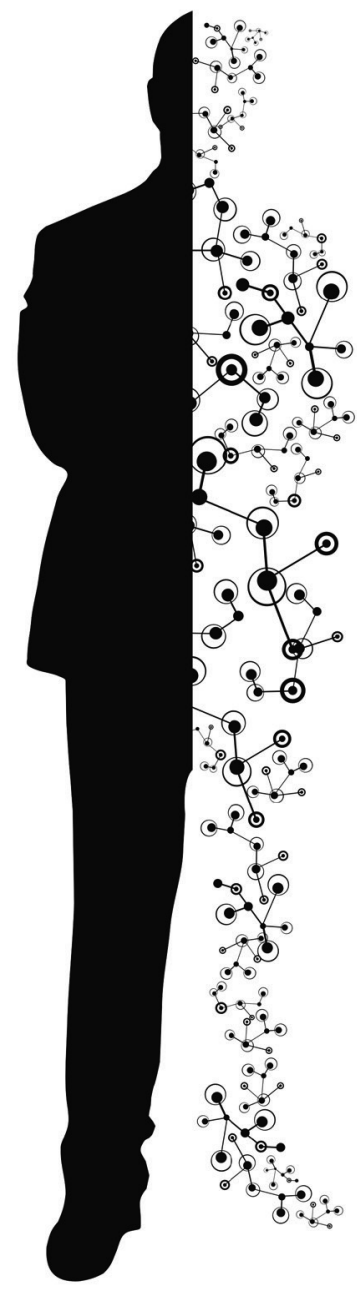




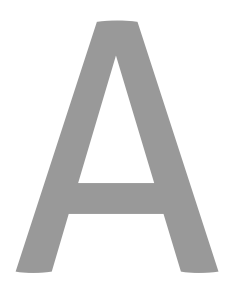

\section{Appendix}

Acknowledgements - Dankwoord

List of publications \& Curriculum Vitae 


\section{Acknowledgements - Dankwoord}

At last, the thesis is finished. It was not always easy, but due to the help, support and love from a whole lot of people it has come to a successful end. I would like to take the opportunity to thank everyone involved!

Professor van der Hulst, Beste René, promotor, hartelijk dank voor de mogelijkheid en het vertrouwen om een onderzoekstraject bij de vakgroep plastische chirurgie te kunnen starten. Het is een traject met veel ups, maar ook downs geworden en uw continue steun hierin heeft veel voor mij betekend. Ik heb bewondering voor de manier waarop $u$ professionaliteit en kunde combineert met plezier op de werkvloer, en dat u ondanks een drukke agenda altijd wel even tijd heeft en daad bij het woord voegt. Ik kijk er naar uit de komende jaren gedurende de opleiding tot plastisch chirurg veel van u te leren.

Professor Kramer, Beste Boris, promotor, "vielen Dank für alles" dekt nauwelijks de lading. Vanaf onze eerste ontmoeting was er een goede energie en voelbaar vertrouwen. Altijd was er ruimte voor overleg, of dit nu in het weekend via videoverbinding, of live op het terras van een goed restaurant was. Uw inspiratie, visie en motivatie hebben een belangrijke bijdrage geleverd aan de totstandkoming van deze thesis. Ondanks het feit dat de thesis nu voltooid is, kijk ik uit naar toekomstige momenten van "nutritional support and life wisdom", alsmede professioneel samen te blijven werken.

Doctor Wolfs, Beste Tim, co-promotor, enorm bedankt voor alles wat $u$ voor me heeft gedaan vanaf het moment dat ik volledig blanco het lab van de kindergeneeskunde binnenkwam tot en met het schrijven van deze thesis. Ik heb enorm veel geleerd van de vruchtbare discussies, kritisch bekijken van de data en het aandraaien van de duimschroeven op momenten waar dat nodig was. $U$ heeft mijn belangen meer dan eens behartigd, waarvoor eeuwige dank. Ik kijk er naar uit onze samenwerking in de toekomst voort te zetten.

Dear members of the assessment committee: chairman prof. dr. F. Ramaekers, prof. dr. med. J. Beier, prof. dr. M. van Griensven and dr. D. Booi. Thank you for taking the time to read and evaluate this thesis and take part in the committee. I am very much looking forward to the defense. 
Doctor Piatkowski, Beste Andrzej, bedankt voor de prettige samenwerking, doortastendheid, klinische input en inzichtelijke commentaren tijdens onze brainstormsessies.

Graag wil ik ook van de gelegenheid gebruik maken om alle co-auteurs van de betreffende papers hartelijk te danken voor hun harde werk en belangrijke rol in het mogelijk maken van deze thesis. Daniel Molin, bijzonder fijn dat we gebruik mochten maken van de faciliteiten in het Fysiologie lab en fantastisch om met $u$ te kunnen sparren omtrent het onderwerp angiogenese. Todor, je bent een meta-analyse beest. Het was heel fijn om met je samen te werken. Juliette Hommes, je pragmatische en zeer nauwgezette wijze van werken is een sleutel tot succes, dank!

Tot mijn grote plezier heb ik gedurende mijn $\mathrm{PhD}$ traject met verschillende afdelingen gewerkt. Hierbij wil ik het lab Kindergeneeskunde met klem bedanken dat ze mij in hun gelederen hebben gesloten. Nico, Lilian, Leon en Teun, wat was het fijn om met jullie te werken! Jullie hebben mij zeer warm ontvangen, wegwijs gemaakt in het lab, veel technieken geleerd en zijn van onschatbare waarde geweest voor het genereren van experimentele data. In het bijzonder een dankwoord aan Teun, die zich onvermoeibaar heeft ingezet om in rap tempo ELISA's en PCR's te draaien voor onder andere het ES ASC project waardoor je met recht een co-auteurschap verdiende. Helaas ben je er niet meer, maar je glimlach, gezelligheid, optimisme, leergierigheid en in zeer korte tijd verworven vaardigheden zullen nooit vergeten worden.

Daan en Ruth, mijn roomies van het lab. Wat was het een genot om met jullie te kunnen samen werken. Daan, ik heb veel van je geleerd op onderzoeksgebied. De manier waarop jij je onderzoek doet is een voorbeeld voor allen. Je humor en kennis van allerlei realityfeitjes maakten het helemaal af. Ruth, enorme doorzetter, jouw vrolijkheid werkte aanstekelijk. Je hebt inmiddels veel bereikt en ik wens je alle succes in de toekomst!

Maria, Monique, Luise, Helene, Ilse en Charlie, (ex)mede promovendi. Ondanks dat we niet direct samen hebben gewerkt heb ik erg genoten van de momenten die we samen doorbrachten. Heel veel geluk en voorspoed in eenieders carrière.

Matthias, ik vergeet de tijd niet die wij stug doorwerkend zij aan zij hebben doorgebracht in de kelder van de universiteit. You are a life saver. 
Daarnaast was het een groot voorrecht in het lab om de rollen op enig moment om te draaien van zelf student zijn tot het worden van supervisor om opgedane kennis en kunde door te mogen geven. Ik wil Dirk, Danielle, Bernice en Joël hartelijk danken voor de inzet, jullie bijdrage wordt zeer gewaardeerd.

Beste Hans en Eric, enorm bedankt voor de mogelijkheid samen te werken zowel met jullie als persoon als met het bedrijf Neuroplast, een belangrijke nieuwe speler in de wereld van autologe celtherapie voor patiënten met ernstige en tot nu toe onbehandelbare neurodegeneratieve aandoeningen. Ik heb veel respect voor de manier waarop jullie het bedrijf hebben opgebouwd en uitgebreid. Chapeau! Onze trip naar Parijs was er een om nooit te vergeten. Met veel genoegen kijk ik uit naar de resultaten van de lopende en komende klinische trials. Wellicht dat we in de toekomst onze samenwerking kunnen voortzetten. Raf en Leon, bedankt voor goede samenwerking binnen het spinal cord injury project, welke cruciaal is het geweest voor het slagen van dit project.

Een grote en oprechte steunbetuiging gaat uit naar het gehele team plastische chirurgie in de regio. Ooit ben ik begonnen als semi-arts bij de plastische in Viecuri. Ik heb zeer veel kunnen leren in een fantastische werkomgeving. John, een betere start bij de plastische had ik mij niet kunnen voorstellen, dank voor je geduld, kennis, onderwijs en prettige manier van werken. Hierna volgde een periode van fulltime onderzoek. Toen ik na voltooiing van deze fase weer de kliniek in ging als ANIOS plastische chirurgie in Zuyderland voelde dat als een verse start. Een te gek gevoel, maar tegelijk ook spannend. Ik had namelijk geleerd om te denken en werken als een echte onderzoeker, maar een klinische dokter zijn is een heel ander verhaal. Het team heeft me fantastisch opgevangen en geleerd om mijn eerste zelfstandige poli's en kleine ingrepen te doen. Dit kwam goed uit bij de overstap naar locatie Sittard, waar de programma's af en toe toch net een tikkeltje drukker waren. Ondanks de lange dagen kwam ik elke ochtend met veel plezier naar het werk. Dank jullie wel Marleen, Tom, Darren, Michelle, en Mark! Tot snel.

Als ANIOS plastische chirurgie in het MUMC+ heb ik een heel andere kant van de plastische chirurgie mogen ervaren. Het was een flinke transitie in onder andere casuïstiek en bijbehorende ingrepen, ziekenhuiscultuur, logistiek en ICT systemen. Hoewel het best even wennen was, lag hier een uitstekende mogelijkheid mezelf te ontwikkelen, zowel als mens 
en als dokter. Ik wil de staf alhier, René, Andrzej, Stefania, Shan, Thomas, Xavier en Heidi dan ook uit de grond van mijn hart danken voor de mooie en waardevolle tijd, het aanscherpen van mijn klinische vaardigheden en de strakke aansturing en feedback. Ik kijk uit om over een tijdje, na de vooropleiding Chirurgie te Zuyderland, als AIOS plastische chirurgie nog veel meer te kunnen leren van jullie!

Carlo en Eric, jullie kennis en kunde is een verrijking voor de afdeling. Met veel genot leer ik van jullie een kant van het vak die erg belangrijk is, maar wellicht niet altijd op die manier gewaardeerd wordt.

Dan de assistenten uit de regio, Juliette, Laura, Rutger, Renee, Chao, Anouk, Mintsje, Nadine, Tim, Lotte, Jamilla en Rachel, collega's uit Eindhoven Martijn, Bas, Carlijn, en Anton en uiteraard de inmiddels oud-assistenten turned plastisch chirurgen Kim, Vincent, An, Marijke, Patrick, Alexander, Emiel, Tiara en Lisette. Jullie zullen en hebben een belangrijke rol gespeeld in mijn opleiding, maar zorgden ook dat het hartstikke gezellig bleef. Duizendmaal dank!

Niet te vergeten de collega-onderzoekers die nog in hetzelfde wetenschappelijke bootje zitten: Sander, Ennie, Joost, Renée en Hansje. Jullie zijn toppers, hou vol en vul dat boekje! Bjorn, stiekem hoor jij hier natuurlijk ook bij. Naast een strakke regie op de operatiekamer, heb je de onderzoeksteugels ook al in bedwang.

Heel belangrijk als assistent zijn goede semi-artsen. De interactie die voortkomt uit aansturing en daarop nieuwsgierige vragen terug, zorgt ervoor dat je kritisch blijft nadenken en daarmee je eigen functioneren naar een hoger niveau tilt. Samenwerking is essentieel. Zeker in de drukke periode waarin bepaalde delen van dit proefschrift afgemaakt moesten worden was het fijn om een goed team te hebben. Vandaar mijn grote dank aan Inge, Maud, Lars en Yasmin, you rock! Succes allen in de (nabije) toekomst.

Ook een bijzonder dankwoord aan Mariëlle, Petra en Jolanda. Altijd klaar om van alles en nog wat te regelen, praktische problemen op te lossen, spoed patiënten in te plannen en het naast dat alles ook nog eens leuk te houden.

Tevens een grote steunbetuiging aan de groep mensen die ons werk als dokter überhaupt mogelijk maken: de polimedewerkers, (wond)verpleegkundigen en OK assistentes van 
Viecuri, Zuyderland en het MUMC+. Dank voor de prettige samenwerking. Laten we die nog lang zo voortzetten!

Onderzoek doe je zelden alleen. Ik ben erg dankbaar voor de ondersteuning van NUTRIM en de coöperatieve sfeer binnen Universiteit Maastricht, die meer dan eens heeft geleid tot het uitwisselen van ervaringen, inwinnen van specifieke (technische) kennis of het delen van apparatuur en materialen. Ik wil graag de proefdierfaciliteit in Maastricht bedanken voor de op piekdagen zeer intense samenwerking, strakke uitvoering en het algehele gevoel er voor mens en dier het beste uit te willen halen. Een bijzonder dankjewel aan Saskia, Richard, Inger en Denise. Hiernaast heeft ook de afdeling Fysiologie een goede bijdrage geleverd. Mick, bedankt voor de ondersteuning en hulp bij het ES project! Verder was deze thesis niet mogelijk geweest zonder samen te werken met andere afdelingen zoals Heelkunde, Neuroscience, M4I en MRLN.

Professor Barker, John, thank you for the opportunity to join the Frankfurt Initiative for Regenerative Medicine as a research fellow. Thanks to everyone in the team for teaching me different lab techniques, including electrical stimulation. Vincent, I enjoyed your fascinating views and our conversations on LARPing. Zach, bedankt voor de tijd die we samen hebben doorgebracht in Frankfurt, hopelijk lukt het je snel ertussen te komen bij de plastische chirurgie!

And last but certainly not least, een zeer oprecht en gemeend dankjewel aan familie en vrienden. Een promotietraject verloopt slechts zelden van een leien dakje, en dat was bij mij ook zeker niet het geval. Het heeft een aardige tijd geduurd en in die periode zijn er natuurlijk ook wat minder leuke momenten geweest. Het zijn juist die momenten waarop ik altijd bij jullie terecht kon. Of het nu gaat om een glaasje wijn of een desperados, barbecue, feestje of vakantie naar waar dan ook, dit zijn de momenten die tellen. Voor iedereen die ik tot mijn vriendengroep mag rekenen, het volgende: “...is everybody happyyyyyy?” En proost op nog veel meer mooie momenten in de toekomst.

Lieve mam, wat heb jij het toch maar fantastisch voor elkaar gekregen voor mij en Jop. We zijn nooit iets tekort gekomen en altijd stond je voor ons klaar. Dikwijls heb je jezelf hierbij weggecijferd, terwijl jij zelf ook keihard aan de weg timmerde. Met veel trots heb je de ontwikkeling van onze (school)carrières gevolgd, en soms maakte je je misschien een beetje 
zorgen, maar nu is het moment daar dat beide zoons gepromoveerd zullen zijn. Moeder bedankt voor alles, ik ben trots op jou.

Beste Pap en Suus, bedankt voor alle support door de jaren heen. Het doorzettingsvermogen en harde werken komt gelukkig van geen vreemde. Ook jullie toonden veel interesse in de stand van zaken en waren niet vies van een hapje of drankje om bij te kletsen met bijbehorende gezelligheid. Maar pap, nu met het afmaken van dit boekje gaat een scala aan smoesjes verdwijnen en wordt het wel tijd dat we echt gaan sporten.

Mijn kleine grote broertje Jop. Beetje jammer dat je mijn idee hebt gekopieerd om plastisch chirurg te worden en het dan ook nog voor elkaar krijgt eerder te promoveren en aan je opleidingstraject te beginnen. Grapje natuurlijk, al hebben wel veel mensen hier naar gevraagd. Ik ben juist enorm trots op wat je bereikt hebt en zeer zeker nog gaat bereiken. Je doorzettingsvermogen, gecombineerd met precisie, top $3 \%$ attitude, humor en social skills gaan je nog ver brengen. Oh, en je fameuze danspasjes. Het is erg fijn om iemand dichtbij te hebben die dezelfde weg bewandelt, zodat we vaak en makkelijk kunnen sparren. Bij onze bruiloft was je "best man" en dat blijf je ook. Ik kijk uit naar onze toekomstige samenwerking bro!

Opa Harry en oma letje, opa Jos en oma Truus. Hartelijk dank voor de talloze fijne momenten die Jop en ik bij jullie hebben mogen doorbrengen. Altijd stonden jullie voor ons klaar. Het is zeer spijtig dat onze beide opa's onze promoties hebben moeten missen, want wat waren ze altijd trots, en iedereen mocht dat horen.

Beste Frans, ik kan je niet genoeg bedanken voor de mooie tijden en vrolijke herinneringen. Van spaarpot vol kleingeld voor de kermis tot mijn eerste (en volgende) auto's. Je hebt me veel geleerd over allerlei facetten van het leven en hebt me meer dan eens uit de brand geholpen. Jop zei het al, maar ook ik meen het als ik zeg dat ik hoop ooit eens wat terug te kunnen betekenen.

Lieve schoonfamilie, John, Shireen, Mitchell, Jordy, oma Riet en oma Poemie. Bedankt voor jullie warmte, support en dat jullie altijd voor ons klaar staan, no matter what. Naast alle gezelligheid en klushulp zijn jullie er ook voor onze kids. Ik kan geen betere schoonfamilie wensen! 
Mees en Miles, mijn lieve kids. Wat hou ik ontzettend veel van jullie en wat doet een glimlach soms goed na een drukke dag. Mees, ik ben enorm trots op je, zelfs als we weleens mopperen. Je doet het fantastisch op school, bent erg begaan met de medemens, hebt een heerlijk karakter en zorgt heeeel goed voor je broertje Miles. Miles, lekker mannetje, wat is het een genot om je te zien opgroeien. Je bent een kleine boef. Al sta je nu net pas op het moment om zelfstandig te gaan lopen, ik kan niet wachten om samen echte mannendingen te gaan doen.

Lieve lieve Djin, het zit er eindelijk op! Jij bent van allen de grootste motivator geweest, die altijd achter me heeft gestaan, maar die in de loop van het promotietraject ook het meeste heeft moeten verduren. De LAT-relatie toen ik in Frankfurt zat, de piekmomenten voor deadlines, frustraties bij misgelopen experimenten, alle momenten waar rekening mee gehouden moest worden zoals de tripjes naar het lab voor tijdspecifieke experimenten en ga zo maar door. Het lastige aan onderzoek is dat je werkdag nooit echt af is, er is altijd nog wel iets dat kan gebeuren of in mijn geval dat in mijn hoofd rond speelde. Dat heeft de nodige quality time gekost en die wil ik toch nu wel echt weer gaan inhalen. Dat heet, als jij in het vervolg tijd hebt, want met je nieuwe baan staat je een grote uitdaging te wachten en voor mij begint ook weer een nieuw avontuur. Maar, zoals je er voor mij bent geweest zo wil ik jou ook steunen. Je bent een top moeder en een fantastische echtgenote. Lieverd, ik hou van je en ben trots op je. Cheers to the future, op nog vele vele mooie jaren samen! 


\section{List of publications}

- Jip Beugels, Daniel G.M. Molin, Daan R.M.G. Ophelders, Teun Rutten, Lilian Kessels, Nico Kloosterboer, Andrzej A. Piatkowski de Grzymala, Boris W.W. Kramer, René R.W.J. van der Hulst, Tim G.A.M.Wolfs. Electrical stimulation promotes the angiogenic potential of adipose-derived stem cells. Sci Rep. 2019 Aug 19;9(1):12076.

- Munter JP de*, Beugels J*, De Munter S, Jansen L, Cillero-Pastor B, Moskvin O, Brook G, Pavlov D, Strekalova T, Kramer BW, Wolters ECh. Standardized human bone marrow-derived Stem Cells Infusion Improves Survival and Recovery in a rat model of Spinal Cord Injury (*contributed equally). J Neurol Sci. 2019 Jul 15;402:1629.

- Beugels J, De Munter JPJM, Van der Hulst RRWJ, Kramer BWW, Wolters Ech. Efficacy of Different Doses of Human Autologous Adult Bone Marrow Stem Cell Transplantation on Angiogenesis in an Immune Deficient Rat Model with Hind Limb Ischemia. J Stem Cell Res Dev 2019; S1002.

- Krastev TK, Beugels J, Hommes J, Piatkowski A, Mathijssen I, van der Hulst R. Efficacy and Safety of Autologous Fat Transfer in Facial Reconstructive Surgery: A Systematic Review and Meta-analysis. JAMA Facial Plast Surg. 2018 Sep 1;20(5):351360.

- Beugels J, Hommes JE, Balthasar AJR, van der Hulst RRWJ, Piatkowski de Grzymala AA. Autologous Fat Transfer as a Treatment for Peripheral Neuropathic Pain without Apparent Cause. Plast Reconstr Surg Glob Open. 2018 Aug 16;6(8):e1905.

- Tanaydin V, Beugels J, Andriessen A, Sawor JH, van der Hulst RRWJ. Randomized Controlled Study Comparing Disposable Negative-Pressure Wound Therapy with Standard Care in Bilateral Breast Reduction Mammoplasty Evaluating Surgical Site Complications and Scar Quality. Aesthetic Plast Surg. 2018 Aug;42(4):927-935.

- Beugels J, van der Hulst R.R.W.J., Piatkowski de Grzymala A. Atypische faciale pijn na tandextractie: behandeling door middel van lipofilling. NTPC. 2015 Jan, 1: 42 


\section{Curriculum Vitae}

Jip Beugels was born on the $15^{\text {th }}$ of February 1989 in Brunssum, the Netherlands. He attended the Gymnasium at secondary school Sint-Janscollege in Hoensbroek and graduated summa cum laude in 2007. Already determined to become a plastic surgeon, he enrolled at Maastricht University the same year to start medical school. From the $3^{\text {rd }}$ year onwards he assisted several research projects. In the final year of medical school he

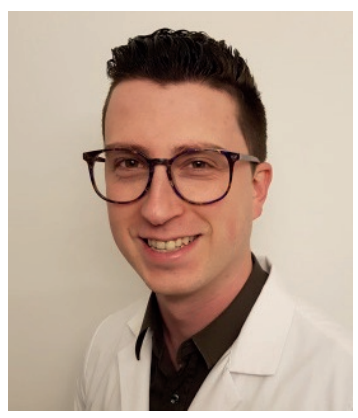
did a combined internship in plastic surgery at Viecuri Medical Center, where clinical rotations were alternated with a scientific internship. After graduation, he did a research fellowship at the Frankfurt Initiative for Regenerative Medicine in Frankfurt am Main, Germany. After a year Jip returned to join the department of plastic surgery at Maastricht University Medical Center to do his PhD research. During this time he attended numerous conferences, gave multiple presentations and won a poster prize at the British Association of Aesthetic Plastic Surgeons meeting in London in 2014 and the Pélerin Pitch prize in Maastricht in 2017. In 2017 Jip became a resident in plastic surgery at Zuyderland Medical Center and moved in 2019 to Maastricht University Medical Center. In October 2020 Jip will continue his plastic surgery residency training with a residency in General Surgery at Zuyderland Medical Center. 

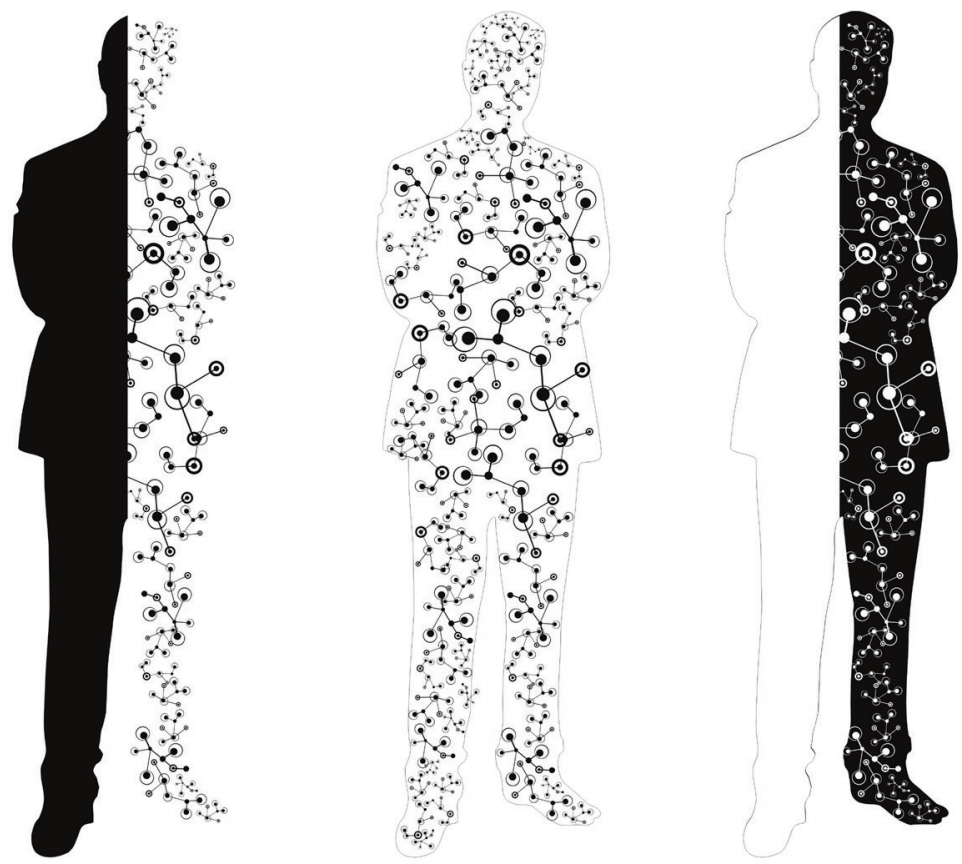